\title{
Essays in Risk Management
}

by

Mahdi Yadipur

\author{
A thesis \\ submitted to the Victoria University of Wellington \\ in fulfilment of the \\ requirements for the degree of \\ Doctor of Philosophy \\ in Finance. \\ Victoria University of Wellington
}

2020 



\begin{abstract}
This thesis consists of five chapters that examines risk and uncertainty within two frameworks: foreign exchange market and real options. The first chapter is a preliminary part that overviews the structure of thesis. In the second chapter, I examine the impact of scheduled macroeconomic announcements on realised variance in the Canadian dollar/US dollar foreign exchange market. Information shocks as a whole are made up of public information shocks and private information shocks. I measure the public information shocks from the analyst forecast surprise and the private information shocks from volatility sensitivity to liquidity variables. I find that the realised variance is driven mainly by the latter rather than the former. However, my results for the most important announcements are not significant, which might be due to these being well-analysed publicly. Spread, as a proxy of private information shocks, is the most important liquidity measure, showing a significant increase around the arrival of announcements. My results are robust to joint effects of liquidity variables, considering announcements throughout the day (times other than 8:30 announcement), alternative measures of volatility (absolute return and modified absolute return), evaluation of announcements for US and Canada separately, examine the impact of surprise in model, and the economic classification of announcements.

In the third chapter, I aim to evaluate risk and uncertainty using real options technique. I develop a framework to evaluate representative agents' behaviour in a real options switching framework. I set up three models with revertible switching process under uncertainty and solve these using the alternating direction implicit algorithm. The models break down
\end{abstract}


into: cash-cost model, cash-time model, and projection model. The cashcost model captures the cash expenses of switching whereas the cash-time model not only captures the cash cost but also the exact time cost, which is critical in horticulture. The projection model presents an approximation of cash-time model that has less computational complexity. The results of my sensitivity analyses indicate that increases in cost, time, volatility, drift, and discount rate have negative impacts on the switch frequency. If the correlation between two crops is positive, it has negative impacts on switch frequency, otherwise it has positive impacts. Differences between the models are more pronounced over longer periods.

In the fourth and fifth chapters, I extend the cash-time model from chapter three to evaluate orchardists' behaviour in the Hawke's Bay region. Chapter four examines the dataset thoroughly and provide a statistical review of orchards that will be modelled in chapter five. Orchardists have the incentive to switch from one type of apple to another as the apple profits change. In my model, orchardists have the option to carry on with the existing apple trees or to switch to competing apple types by uprooting the existing apple trees and planting new ones or grafting on the existing rootstock. The uprooting strategy is relatively expensive but is instantaneous, and results in young (unproductive) apple trees with a long life ahead of them. In contrast, the grafting strategy is less expensive and faster but continues with old trees. I compute the optimal land value at each age of apple trees from one-year to 33-years old. My results show that grafting is the optimal strategy when trees are young, whereas planting becomes optimal when they are old. Examining the apple dataset, I find that orchardists are biased against uprooting and grafting relative to my predictions. The deviation from what my model proposes and what orchardists follow in reality might be due to the assumption of my model and possible factors in the orchards that my model does not capture. My results show that the deviation from optimal policy for small orchardists is not significantly different from large orchardists. 


\section{Acknowledgements}

I would like to express my sincere appreciation to my primary supervisor, Associated Professor Toby Daglish, for his amazing support and excellent advice throughout my PhD study. Toby opened a new opportunity for my research, has expanded my thinking and has significantly helped me on my path to a better future. I am also thankful to Yigit Saglam, my secondary supervisor, for his insightful and constructive comments. I also had the opportunity to be supervised by Ingrid Lo for the first year of my $\mathrm{PhD}$ studies and I am grateful of her support and guidance.

My highest appreciation goes out to my parents especially my mother who sacrificed her comfort for a better life for her children. She is my hero and her prayers are a blessing for me. I thank my brother Mohammad and my sister Samaneh for supporting me emotionally and spiritually throughout the time I have been away from home. I am thankful to all my friends and others that have supported me. You have a special place in my heart and these words are unable to express my appreciation to all of you.

I am thankful to Victoria University of Wellington and the School of Economics and Finance in particular for providing me a full scholarship and supporting me by all means necessary including the amazing facilities that have allow me to study with peace of mind. 


\section{Contents}

1 Preliminaries 1

1.1 Abstract ....................... 2

1.2 Thesis Structure . . . . . . . . . . . . . . . . 2

2 News and FX Market $\quad 7$

2.1 Introduction . . . . . . . . . . . . . 8

2.2 Data and Descriptive Statistics . . . . . . . . . . . . 12

2.2.1 The Canadian FX Data . . . . . . . . . . . . . . . . 12

2.2.2 Macroeconomic Announcement Data . . . . . . . . . 13

2.2.3 Constructing Variables . . . . . . . . . . . . . . . 18

2.3 Empirical Analysis . . . . . . . . . . . . . . . . . 24

2.3 .1 The Model . . . . . . . . . . . . . . . . . . . . 24

2.3 .2 Regression Analysis . . . . . . . . . . . . . . 26

2.4 Robustness and Extensions . . . . . . . . . . . . . . . 31

2.4.1 Realised Variance Regression Without Lag . . . . . . 34

2.4.2 Realised Variance in the US and Canadian Market Separately .................. . . 34

2.4.3 Surprise Variable Analysis . . . . . . . . . . . . . . 40

2.4.4 Alternative Measures of Volatility . . . . . . . . . . . 43

2.4.5 The Effect of All Liquidity Variables Jointly . . . . . . 51

2.4.6 The Effect of News at Times Other Than at 8:30 . . . 52

2.4.7 The Magnitude of Liquidity Variable Changes . . . . 56 
2.4.8 Risk Averse/Seeking Trader in the Market . . . . . . 58

2.4.9 Realised Variance in Economic Samples . . . . . . . . 59

2.5 Summary and Conclusion . . . . . . . . . . . . . 68

2.5.1 Summary of All Regression Analysis . . . . . . . 68

2.5 .2 Conclusion . . . . . . . . . . . . . . . 71

3 Optimal Enterprise Use Switching Policy 73

3.1 Introduction . . . . . . . . . . . . . . . . 74

3.2 Methodology . . . . . . . . . . . . . 78

3.2.1 Solution Method . . . . . . . . . . . . . . . . 84

3.3 Numerical Illustration . . . . . . . . . . . . . . . . . 90

3.3.1 Simulation and Bootstrap . . . . . . . . . . 90

3.3.2 Baseline Parameters and Scenarios . . . . . . . . . 92

3.3.3 Optimal Exercise Frontiers . . . . . . . . . . . . 94

3.3.4 Sensitivity Analysis . . . . . . . . . . . . . 100

3.4 Conclusion . . . . . . . . . . . . . . . . 116

4 Data Analysis $\quad 119$

4.1 Data Statistics . . . . . . . . . . . . . . . 120

5 A Complex Switching Option Model 141

5.1 Introduction . . . . . . . . . . . . . . . . . . . 142

5.2 Apple Orchard Characteristics . . . . . . . . . . . . 146

5.2.1 Horticulture Sector . . . . . . . . . . . . . 146

5.2.2 The Uproot-Plant Versus The Graft . . . . . . . . . . . 148

5.3 Methodology . . . . . . . . . . . . . . . . . 149

5.4 Empirical Results . . . . . . . . . . . . . . . . . 156

5.4.1 Sensitivity Analysis . . . . . . . . . . 162

5.5 Conclusion . . . . . . . . . . . . . . 167 


\section{List of Tables}

2.1 Summary Statistics of Pre-scheduled Macroeconomic Announcements Survey (US). . . . . . . . . . . . . . . . 16

2.2 Summary Statistics of Pre-scheduled Macroeconomic Announcements Survey (Canada). . . . . . . . . . . . . 17

2.3 Difference in Difference t-test Results for News Days vs Nonews Days and before vs after Announcements. . . . . . . . 25

2.4 Realised Variance Unit Root Test Before and After 8:30 . . . 27

2.5 Realised Variance at 8:30 Announcements Using the Full Sample. . . . . . . . . . . . . . . . 30

2.6 Realised Variance at 8:30 Announcements Using the RI Sample. . . . . . . . . . . . . . . 32

2.7 Realised Variance at 8:30 Announcements Using the MI Sample. . . . . . . . . . . . . . . 33

2.8 Realised Variance at 8:30 Announcements Using the Full Sample, without Lag. . . . . . . . . . . . . . . . 35

2.9 Realised Variance at 8:30 Announcements Using the RI Sample, without Lag. . . . . . . . . . . . . . . 36

2.10 Realised Variance at 8:30 Announcements Using the MI Sample, without Lag. . . . . . . . . . . . . . . . . 37

2.11 Realised Variance at 8:30 Announcements in US, Using the Full Sample. . . . . . . . . . . . . . . . . . . . . . 38 
2.12 Realised Variance at 8:30 Announcements in CA, Using the Full Sample. . . . . . . . . . . . . . . . . . 39

2.13 Realised Variance at 8:30 Announcements Using the Full Sample with no Surprise. . . . . . . . . . . . . . 41

2.14 Realised Variance at 8:30 Announcements Using the Full Sample with Positive and Negative Surprises. . . . . . . . . 42

2.15 Absolute Return at 8:30 Announcements Using the Full Sample. . . . . . . . . . . . . . . . 44

2.16 Absolute Return at 8:30 Announcements Using the RI Sample. 45

2.17 Absolute Return at 8:30 Announcements Using the MI Sample. . . . . . . . . . . . . . . 46

2.18 Modified Absolute Return at 8:30 Announcements Using the Full Sample. . . . . . . . . . . . . . . . . . . . . . . . 48

2.19 Modified Absolute Return at 8:30 Announcements Using the $R I$ Sample. . . . . . . . . . . . . . . . . . . . 49

2.20 Modified Absolute Return at 8:30 Announcements Using the MI Sample. . . . . . . . . . . . . . . . . 50

2.21 The Effect of All Liquidity Variables Jointly on Realised Variance. ...................... 53

2.22 The Effect of News at Times other than 8:30 on Realised Variance. . . . . . . . . . . . . . . . . 55

2.23 The Magnitude of Changes after Announcements. . . . . . . 57

2.24 Market Condition for Risk Averse/Seeking Trader. . . . . . . 60

2.25 Pre-scheduled Macroeconomic Announcements Survey in 4 Groups in US. . . . . . . . . . . . . . . . . . 61

2.26 Pre-scheduled Macroeconomic Announcements Survey in Canada. . . . . . . . . . . . . . . 62

2.27 Realised Variance Using the Output Sample. . . . . . . . . . 63

2.28 Realised Variance Using the Prices Sample. . . . . . . . . . . 65

2.29 Realised Variance Using the Employment Sample. . . . . . . 66

2.30 Realised Variance Using the Rate Decision Sample. . . . . . . 67 
2.31 Summary of Significant Results. . . . . . . . . . . . . . . . . 70

3.1 Benchmark and Sensitivity Analysis Scenarios . . . . . . . . 93

3.2 Benchmark Switching Analysis . . . . . . . . . . . . . . . 95

3.3 Cost and Time Switching Analysis . . . . . . . . . . . . . . 101

3.4 Volatility and Drift Switching Analysis . . . . . . . . . . . . 104

3.5 Discount Rate Switching Analysis . . . . . . . . . . . . . . 109

3.6 Correlation Switching Analysis . . . . . . . . . . . . . . 111

3.7 Changes in the Switching Behaviour When the Baseline Parameters Changes in Each Scenario. . . . . . . . . . . . . 114

3.8 Changes in Switching Behaviour Across Three Models When Product 2 Parameters change. . . . . . . . . . . . . . . 115

4.1 Allocated Land to apple varieties in New Zealand . . . . . . 121

4.2 Allocated Land to apple varieties in different Regions in New Zealand . . . . . . . . . . . . . . . . . . . . 122

4.3 The Number of Switches Among Apple Varieties in All Regions. . . . . . . . . . . . . . . . . 124

4.4 Allocated Land Based on Switches in All Regions . . . . . . 125

4.5 Allocated Land to apple varieties in Hawke's Bay . . . . . . 126

4.6 The Number of Switches Among Apple Varieties in Hawke's

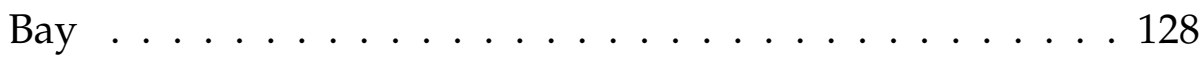

4.7 Apple Prices Over Time Period 2006-16. . . . . . . . . . . . . 129

4.8 Clustering Apple Varieties. . . . . . . . . . . . . . . . . 133

4.9 Age Groups of Switches in All Region. . . . . . . . . . . . . 135

4.10 t-test Results for Switches Based on Trees' Age. . . . . . . . . 136

4.11 The DGM Approach to Estimate Discount Rate. . . . . . . . 138

4.12 The CAPM Approach to Estimate Discount Rate. . . . . . . . 139

5.1 Apple Trees Cash Flows Throughout Their Lifetime Using the Uproot-Plant and the Graft Strategy. . . . . . . . . . . . 149

5.2 Initial Cost to Establish an Orchard. . . . . . . . . . . . . . . 150 
5.3 Queen and Braeburn Apple Types Parameters. . . . . . . . . 158

5.4 Sample Results of Dynamic Optimisation. . . . . . . . . . . 159

5.5 Conditional Logistic Regression Results. . . . . . . . . . . . . 162

5.6 Uproot-Plant Cash Cost Change Sensitivity. . . . . . . . . . . 163

5.7 Uproot-Plant Time Cost Change Sensitivity. . . . . . . . . . . 164

5.8 Drift Change Sensitivity. . . . . . . . . . . . . . . . . . . 165

5.9 Volatility Change Sensitivity. . . . . . . . . . . . . . . 166

5.10 Discount Rate Change Sensitivity. . . . . . . . . . . . . 166 


\section{List of Figures}

3.1 Optimal Exercise Frontier for Baseline Parameters. . . . . . . 97

3.2 Optimal Exercise Frontier for Baseline Parameters but Negative Correlation. . . . . . . . . . . . . . . . . 99

3.3 Optimal Exercise Frontier when Time Changes (Scenario 1.B).103

3.4 Land Value with Product 1 in the cash-time model when Volatility Increases. . . . . . . . . . . . . . . 106

3.5 Land Values Before (baseline) and After Change in Volatility in the cash-time model. . . . . . . . . . . . . . 107

4.1 Apple Prices Path Over Time Period 2006-16. . . . . . . . . . 130

4.2 Ratio of Braeburn Price to Queen Price Over Time Period 2006-16. . . . . . . . . . . . . . . . . 131

5.1 Optimal Land Use Policy. . . . . . . . . . . . . . . . . . . 160 
Chapter 1

\section{Preliminaries}




\subsection{Abstract}

This thesis evaluates risk and uncertainty by employing two approaches: market microstructure and real options framework. The second Chapter is an application of market microstructure approaches to evaluate realised variance being affected by the arrival of scheduled macroeconomic announcements. The third Chapter evaluates uncertainty using the real options approach. I construct a real options problem by deriving a partial differential equation for two competing products and solving it using the alternating direction implicit algorithm. Chapter four examines orchard data in the Hawkes Bay region and provides a statistical overview to evaluate orchardists behaviour in the following Chapter. In Chapter five, I develop the general model from Chapter three to a more complex and dynamic framework and examine if orchardists optimise their lands value according to the real options analysis.

\subsection{Thesis Structure}

This thesis evaluates risk and uncertainty in two approaches: (1) market microstructure and (2) real options. In the first approach, I evaluate the impact of macroeconomic announcements on realised variance in the Canadian foreign exchange market. Volatility shocks in the foreign exchange market are either due to having access to public information or private information. As a result, traders send bid or ask quotes in a wide range of execution aggressiveness. I use a valuable dataset that includes all the quotes behind the best quote that enables us to make a better analysis of what occurs behind the scenes. In this paper, liquidity variable (spread at the best quote, depth at the best quote and behind the best quote, absolute order flow, and absolute order imbalance) sensitivity is a proxy for private information shocks whereas financial analyst surprise at the ar- 
rival of public announcements in the market is a proxy for public information shocks. The former measure is derived from the Canadian FX market dataset and the latter from Bloomberg. After initial evaluation of volatility before and after the arrival of macroeconomic announcements, I set up my regression model to examine the sensitivity of realised variance to my explanatory variables. My results indicate that private information explains realised volatility better than public information. Also, spread, as a proxy of private information, reveals more significant results than the other variables. I break the pre-scheduled macroeconomic announcements into three samples: Full, Relatively Important, and Most Important samples. My results show that the first two samples provide more significant results than the last sample and this might be due to extensive public information about the Most Important announcements. As robustness checks, I test the impact of news at times other than 8:30, the effect of all liquidity measures jointly, the impact of macroeconomic announcements in US and Canada separately, a detailed analysis on surprise, an economic classification of announcements and rerun the model, and the magnitude change of liquidity measures in smaller intervals of three 5-minute intervals before and after the 8:30 announcement, between news days and no-news days. Overall, my results show that spread at the best quote and absolute order flow are the main liquidity measures with more significant results, confirming spreads importance.

The thesis proceeds with risk and uncertainty evaluation using the real options frameworks. The third Chapter constructs a real options switching problem under uncertainty and reversibility (the ability to go back to an original product) to optimise the switching between two competing products. The assumptions are: (1) products have an infinite lifetime and (2) there is only one switching strategy (removing the existing product and initiating the other product). In a case of options to switch, investors evaluate a cost-benefit analysis that normally does not follow the Marshallian investment trigger and generates hysteresis. I argue that according to the 
type of activity, the time cost of switching may vary. For example, in livestock farming (e.g. beef and sheep), there is relatively little time cost and the switching process is fast, while with seasonal crops (e.g. beetroot and carrots) there is a time cost of one year, and in horticulture (e.g. apples and grapes) the time cost increases to 5 or 6 years, which is a critical time cost. I set up three models according to each case: a cash-cost model, a cash-time model, and a projection model. I expect that the three models would give qualitatively different outputs. The cash-cost model ignores the time cost while the cash-time model formally measures the time costs and the projection model approximates the time costs using the expected monetary cost of time. I then consider a range of scenarios. In each scenario, I examine the mean number of switches, the probability of one or more switches taking place, and the probability of two or more switches taking place, across the three models and compare with the baseline model. I bootstrap samples and test if my statistics across three models are quantitatively different. Results show that the cash-cost model that is used throughout the literature overestimates the switch frequency. The projection model presents better results compared with cash-cost, but underperforms compared with cash-time due to approximating the time cost, which is very critical in horticulture. The cash-time model computes the exact time cost and its results are significantly different from the projection model when the time of waiting is substantial. The sensitivity results from the cashtime model are significantly different from the projection model at the $1 \%$ level in all scenarios except in the case when volatility is high. Results indicate that increases in cost, time, volatility, drift, and discount rate have negative impacts on the switch frequency. If the correlation between two crops is positive, it has negative impacts on switch frequency, otherwise it has positive impacts.

In Chapter four, I evaluate orchards in the Hawkes Bay region and provide a statistical review of the dataset in terms of land allocation to apple varieties and how this changes over time. I use the measured pa- 
rameters and orchardists actions in the following Chapter.

In Chapter five, I extend my model from the third Chapter and assume: (1) apple trees have a finite productive lifetime similar to what is seen in reality and (2) the model is more complex by having two competing switch strategies: the uproot-plant and the graft strategy. This Chapter not only constructs a complex dynamic real options problem but also compares orchardists choice with the optimal policy that is proposed by the model. In this regard, I examine the behaviour of orchardists in the Hawkes Bay region orchards. In my model, orchardists have the option to plant Braeburn or Queen apples, and they have the incentive to switch from one type of apple to another as revenues change. There are two strategies to switch: the uproot-plant and the graft strategies. The former is a relatively expensive and slow process but results in a rejuvenated orchard, whereas the latter is less expensive and faster but continues with existing apple trees. Hence, I keep track of apple tree age and optimise the land use value under uncertainty and reversibility. Results comprise land use value when keeping the existing apple trees, switching to the competing apple type by the uproot-plant strategy, and switching by grafting. The optimal land value is the maximum of these three values for each apple type and at each node of each age. In results, the optimal land use value (what my model proposes) is compared with what orchardists actually choose. I employ a conditional logit model to examine the difference between the optimal and actual behaviours.

There are situations in my dataset where orchardists' actions do not match the optimal land use policy proposed by my real options model. According to the current model set up, on average an orchardist with a hectare land area loses $0.5 \%$ of the corresponding net revenue of land value that by assuming the same cost of $\$ 50$ for both apple types, varies from $\$ 220.93$ to $\$ 382.43$ for Braeburn and from $\$ 272.98$ to $\$ 693.77$ for Queen. Orchardists have a tendency towards keeping the existing trees and do not 
exercise their options to switch to the competing apple type either through the uproot-plant or the graft strategy. This hysteresis might govern their behaviour as long as there is not any force or a strong incentive to convince them to switch. Any financing or incentive from the government may lead orchardists to switch at the right time and decrease their inertia. My results from the conditional logit model show that orchardists are biased towards inertia. However, these results assume that orchardists do the right thing in the next period of time and theses costs would be small unless they are really in the money. The differences between the optimal land value based on the real options analysis and the actual value that orchardists achieve through their current policy are a proxy for how far they are from the policy advised by my model. However, the model results are based on some assumptions and changing the parameters will minimise the difference between optimal and actual. We test this assertion by changing the feeding parameters in sensitivity analysis section and find lower difference. This shows that orchardists might use different parameters than what we have used in my model. Also, a range of simplifications such as choosing only two apple types and ignoring the fact that orchardists have a portfolio of apple varieties might explain the hysteresis among orchardists that results in difference between theory and practice. Comparing small orchardists with large orchardists reveals the same attitude of being less willing to exercise switching options. 


\section{Chapter 2}

\section{Public and Private Information}

Shocks to Realised Variance in the US/Canadian FX Market 


\subsection{Introduction}

Information asymmetry is one of the main drivers of trading in any market. Information asymmetry in its turn causes adverse selection and price changes. Hence, information shocks and volatility are strongly linked. Information shocks fall into two main categories: public information shocks and private information shocks. Public information shocks are typically measured as financial analyst surprise at the arrival of public announcements in the market ${ }^{1}$ (see Balduzzi, Elton, and Green (2001), Pasquariello and Vega (2007), and Ben-Omrane and Hafner (2015)). On the other hand, private information shocks are a more subtle concept and not so easy to observe or capture. Private information shocks refer to any information that is not publicly available for all market participants. This can come from either direct access to information, or indirect access, such as interpretation capability or forecasting skill of agents in the market.

This chapter examines the limit order book in the Canadian dollar/US dollar currency market. My main contribution is that I use the limit order book to examine asymmetric information impact on liquidity that results in price fluctuations (volatility) in the FX market. I capture the impact of belief dispersion and private information shocks through liquidity measures (namely: spread at the best quote, depth at the best quote, depth at the second best quote, absolute order flow, and absolute order imbalance). At the same time, I use macroeconomic announcements (see Tables 2.1 and 2.2) to measure public information shocks. These two measures allow me to decompose the source of volatility shocks into public and private components.

\footnotetext{
${ }^{1}$ Analyst recommendations (ANR) display a list of analysts' recommendations, price targets, price target time periods, and a consensus rating for a selected equity. The consensus rating is based on analyst recommendations and compiled by Bloomberg reporters and researchers around the world. ANR allows you to determine the latest analyst sentiment and identify which analysts have the most accurate equity ratings (Bloomberg Help).
} 
I conjecture that macroeconomic announcements not only include public information shocks, but also that the interpretation of these data lead to private information shocks. The limit order book is a valuable information source that records the behaviour of participants in the market. I expect that the sensitivity to liquidity measures, as a channel of private information, rises with the arrival of macroeconomic announcements. However, this sensitivity might vary according to the level of importance and how extensively the announcement has been analysed by traders in the market.

Most studies in the market microstructure literature are not specifically based on the limit order book; rather they are extensions of the Kyle (1985) model, which is based on a market maker. However, electronic limit order markets have outperformed dealer markets in many ways. For example, the access to instantaneous execution without the intervention of a market maker. Having said that, the information value of the limit order book and, in particular, the market activity around the arrival of information, remains an open question.

A range of studies shed light on this question and provide some answers (see Foucault, Moinas, and Theissen (2007), Lo and Sapp (2010, 2011), Jiang, Lo, and Valente (2014), Hoffmann (2014), Foucault, Hombert, and Rosu (2016)). These studies conclude that the limit order book contains asymmetric information because limit order submitters can potentially be informed traders. When informed traders submit orders, measures of depth and spread will reflect both private information and public information.

While limit order books are an established part of many markets, the presence of a limit order book for an FX market is particularly interesting. In a traditional FX market, there is no limit order book (i.e. it operates as a dealer market) and order flow serves as a proxy for private information. However, starting in 2000, an electronic limit order book was set 
up by Reuters and Electronic Broking Services (EBS), allowing FX market participants to trade with each other. Traders post orders as either limit orders or market orders, and EBS executes the orders (bids against offers) according to price and time priority. Having the opportunity to observe bids/asks behind the best quotes would enable a trader to gauge the degree of asymmetric information present in the market, and evaluate the risk of market order submission. However, quotes behind the best quoted prices are not observable, and hence they are entirely private information. What is special about my dataset (Reuters D-3000) is that I have access to the dealers' full data including the hidden information (such as behind the best quote) and information on changes in orders (such as adding, deleting, or modifying quotes) in the limit order book. Further, since this is hidden, it could not be taken into account by traders, and hence would not prejudice their market order submission. I contribute to the literature by capturing private information that is carried by limit orders in addition to order flow as proposed in the FX literature. This chapter seeks to address the question of whether private information derived from the limit orders changes the liquidity and moves volatility. The empirical literature shows that public information arrival has a significant impact on volatility (see Bauwens, Ben-Omrane, and Giot (2005), Jiang, Lo, and Valente (2014), and Ben-Omrane and Hafner (2015)). However, this literature is still silent on whether private information, especially that coming from limit orders, affects volatility.

Recent exchange rate studies, instead of relying on macroeconomic determinants, have used the market microstructure approach as a data driven technique to link exchange rate changes to the limit order book as the channel of transferring information (see Evans and Lyons (2002), Lo and Sapp (2011), Lovcha and Perez-Laborda (2013), and Ben-Omrane and Hafner (2015)). However, the limit order book contains more information that can be extracted and used to explain and forecast future price changes due to having access to all the quotes. Lo and Sapp (2011) use 
the electronic order book to evaluate the determinants of order submission strategies (i.e. the choice of limit orders vs market orders) for traders in the Canadian dollar/US dollar currency pair. The aggressiveness of orders and the role of timing in the limit order market is investigated in two other papers (Lo and Sapp, 2008, 2010). I use the same electronic order book (Reuters D-3000) but answer a different question: I assert that volatility can be exacerbated by order aggressiveness. Foucault, Moinas, and Theissen (2007) state that the size of bid-ask spread, which is one of the liquidity measures derived from the limit order book, contains volatility information and can be representative of private information. They also propose that the limit order book includes information about the magnitude of future price fluctuations. Therefore, I incorporate not only the order flow, which has been the main focus of past studies, but also other liquidity measures from the limit order book to explain private information.

The remainder of the chapter is ordered as follows: In Section 2.2, I evaluate the data and descriptive statistics. I examine the Canadian FX data, macroeconomic announcements data, and how to construct the dependent and explanatory variables. In Section 2.3, I set up my main model and, by running the corresponding regression model, and analyse the relationship between realised variance and explanatory variables. In Section 2.4, I present robustness tests to evaluate the primary results of my regression model, namely: realised variance without lag, realised variance in the US and Canadian market separately, surprise variable analysis, alternative measures of volatility, the effect of all liquidity variables jointly, the effect of news at times other than 8:30, the magnitude of liquidity variable changes, and realised variance in economic samples. Finally, in Section 2.5 , I provide a summary of significant results and an overall conclusion. 


\subsection{Data and Descriptive Statistics}

\subsubsection{The Canadian FX Data}

This chapter uses high frequency data for the Canadian FX market, which comes from the Reuters D-3000 proprietary inter-dealer platform. The sample span is from January 4, 2005 to December 30, 2005. The data include information about the electronic order book, both market orders and limit orders, recorded continuously, 24 hours a day. The dataset contains 3085363 observations of transactions in the Canadian FX market. A majority of macroeconomic announcements in this time period occur at 8:30. Following Ederington and Lee (1995), Clare and Courtenay (2001), Sager and Taylor (2011), and Neely and Dey (2010), I focus on a 15-minute interval, because the impact of new information remains much more than normal for approximately 15-minutes after a macroeconomic announcement.

The trades in the dataset are sorted based on the date and the time of entering into the order book or being removed from the order book. The size of trade is recorded, along with an identification code showing whether it is a bid or ask; how much quantity is entered or removed from the order book; and whether it is a limit order or market order. I order the price and quantity of trade in different columns from the best to the worst, in which the "best" bid is the highest price to buy and the "best" ask is the lowest price to sell. Each price has a related quantity. My ordering enables me to evaluate the market condition (e.g. the size, volume, or frequency of trades) behind the best quote.

I use this dataset to measure realised variance (as the dependent variable) and explanatory variables such as spread at the best quote, depth at the best quote, depth at the second best quote, absolute order flow, and absolute order imbalance. The method of measuring these variables is 
explained in Section 2.2.3. I exclude holidays and weekends from the dataset, as there is insignificant trade, and no announcements occur on these days. Comparing the Canadian FX dataset and the pre-scheduled macroeconomic announcements determines news days and no-news days.

I evaluate the market condition using different variables to find the behaviour of the market on news days and no-news days. I exploit the data to measure different factors including trade frequency, limit order frequency, trade volume, trade size, quote size, depth of limit order, and bid-ask spread. Moreover, I not only separate my examination into news days and no-news days, but also before and after the announcement. The results in Section 2.3.1 show that the behaviour of the Canadian FX market on news days is significantly different from no-news days. Similarly, the pattern of these variables is significantly different after the announcement compared to before the announcement.

\subsubsection{Macroeconomic Announcement Data}

Macroeconomic announcements intensify volatility in financial markets and, in particular, foreign exchange markets due to creating asymmetric information among traders. There will be a range of different expectations from the arrival of news in the market. Some news might confirm the prior expectations of some traders whereas it might reject the expectation of other traders. Macroeconomic announcements have different impacts on the price movement and the reaction of agents in exchange markets (Fleming and Remolona, 1999).

The pre-scheduled macroeconomic announcement data is the second dataset I use, along with my main trading dataset. The trade in the Canadian FX market is affected by macroeconomic announcements in both Canada and the US. Therefore, the entire set of macroeconomic announcements by both countries are collected from Bloomberg. The macroeconomic an- 
nouncements vary according to their timing and the effect they have on trading in the market. This dataset helps me to analyse the Canadian FX market in three ways. Firstly, it helps to differentiate news days and nonews days. Secondly, it provides a proxy for surprise to evaluate the impact of public information shocks on volatility. Finally, it enables me to extend my analysis to examine the effect of announcements on volatility according to their type and importance.

The macroeconomic announcements data have different variables including the type of announcement, the date and time of the news release, actual values in the announcements, survey values derived from the expectation of analysts, and the relevance values that indicate the importance of news. ${ }^{1}$ The date and time of macroeconomic announcements is a proxy to separate trading days into news days and no-news days as well as examining the effect of the macroeconomic announcements at a specific time, for instance at 8:30, which is the main interest of this chapter.

The difference between the actual values in the announcements and analyst forecasts measures the amount of surprise in the market. ${ }^{2}$ The literature shows that as the amount of surprise increases, volatility surges (see Andersen, Bollerslev, Diebold, and Vega (2003, 2007), Evans and Lyons (2005), Neely and Dey (2010), King, Osler, and Rime (2013), Ben-Omrane and Hafner (2015), Gomes and Peraita (2016), and Boudt, Neely, Secru, and Wauters (2017)). I also clean the macroeconomic announcements data by deleting announcements with the same nature or if their corresponding forecast value, realised value, or announcement time is not reported.

\footnotetext{
${ }^{1}$ Bloomberg has a search engine to aggregate and generate breaking news and through different feeding sources including web and social media rank the announcements and release it by relevant value indicator.

${ }^{2}$ Analyst recommendations (ANR) display a list of analysts' recommendations, price targets, price target time periods, and a consensus rating for a selected equity. The consensus rating is based on analyst recommendations and compiled by Bloomberg reporters and researchers around the world. ANR allows you to determine the latest analyst sentiment and identify which analysts have the most accurate equity ratings (Bloomberg Help).
} 
After cleaning the announcement data, there are 37 different types of US macroeconomic announcements, along with 16 different types of Canadian macroeconomic announcements during the estimation period.

The main focus of this chapter is to study the impact of the 8:30 announcements on the Canadian FX market. The impact of announcements at other times is considered in the robustness section. Moreover, I control for the effect of news that overlaps with other announcements by removing contaminated announcements (e.g. I exclude the 9:00 Bank of Canada rate decisions and 9:15 US Industrial Production month-on-month report, as their windows overlap). Hence I test the pure impact of the given announcement, which is not contaminated by the impact of other announcements.

Tables 2.1 and 2.2 report the summary statistics of pre-scheduled macroeconomic announcements in the US and Canada. The first column reports all the pre-scheduled macroeconomic announcements in the US and Canada. The following columns present the related attributes of news, including the source of news, the number of observations of each news item, the time of releases, and the summary statistics including the minimum, maximum, mean, and standard deviation of each indicator. Each event happens on a different date, but the time on a given date remains constant. I convert all times to Eastern Time so that the comparison and examination of trades is consistent. ${ }^{1}$ Tables 2.1 and 2.2 report the timing of news announcements. The majority of pre-scheduled macroeconomic announcements arrive at 8:30, which is why this time is the focus of my study. As noted earlier, I broaden my analysis to all times in the robustness section. The pre-scheduled macroeconomic announcements are different in terms of the relevance value (the importance of news). In line with the literature (especially Pasquariello and Vega (2007)), I separate the

\footnotetext{
${ }^{1}$ The release time of news and the time of trade in the US and Canada are reported according to different time zones (GMT, which is Greenwich Mean Time, for Canada, and EST, which is Eastern Standard Time for US).
} 
TABLE 2.1: Summary Statistics of Pre-scheduled Macroeconomic Announcements Survey (US).

This table provides summary statistics for macroeconomic announcements in the US and Canada in the year 2005. The columns present, in order: the type of macroeconomic announcement in the US, the source of announcement releases (the abbreviation of each source is defined below), the number of observations of each announcement, the time of the announcement, minimum, maximum, mean, and standard deviation of observations in each announcement.

Abbreviations: FR: Federal Reserve, BLS: Bureau of Labour Statistics, BEA: Bureau of Economic Analysis, CB: Census Bureau, DL: Department of Labour, B: Bloomberg, T: Treasury, CBB: Chicago Business Barometer, NAHB: National Association of Home Builders, K: Thousands, B: Billion.

\begin{tabular}{|c|c|c|c|c|c|c|c|}
\hline Event & Source & Obs. & Time & Min & Max & Mean & Std. \\
\hline \multicolumn{8}{|l|}{ US Announcements } \\
\hline Bloomberg Consumer Confidence & B & 10 & 17:00 & -22 & -8 & -14.5 & 4.7668 \\
\hline Building Permits & $\mathrm{CB}$ & 12 & 08:30 & 1985 & $2160 \mathrm{~K}$ & $2077.9 \mathrm{~K}$ & $49.8 \mathrm{~K}$ \\
\hline Business Inventories & $\mathrm{CB}$ & 12 & 08:30 & 0.0010 & 0.0090 & 0.0038 & 0.0024 \\
\hline CPI MoM & BLS & 12 & 08:30 & -0.0040 & 0.0090 & 0.0025 & 0.0033 \\
\hline Capacity Utilization & FR & 12 & 09:15 & 0.7890 & 0.8030 & 0.7953 & 0.0035 \\
\hline Change in Nonfarm Payrolls & BLS & 12 & 08:30 & $-150 K$ & $225 \mathrm{~K}$ & $159.3 \mathrm{~K}$ & $101.1 \mathrm{~K}$ \\
\hline Chicago Purchasing Manager & CBB & 12 & 10:00 & 52 & 62.500 & 58.617 & 3.289 \\
\hline Conf. Board Consumer Confidence & $\mathrm{CB}$ & 12 & 10:00 & 88 & 106.30 & 99.050 & 5.706 \\
\hline Construction Spending MoM & $\mathrm{CB}$ & 12 & 10:00 & 0.0030 & 0.0070 & 0.0049 & 0.0011 \\
\hline Consumer Credit & FR & 12 & 15:00 & $4.1 \mathrm{~B}$ & 10B & $6.4 \mathrm{~B}$ & $1.6 \mathrm{~B}$ \\
\hline Continuing Claims & DL & 50 & 08:30 & $2573 K$ & $2939 K$ & $2664.7 \mathrm{~K}$ & $93.2 \mathrm{~K}$ \\
\hline Current Account Balance & BEA & 4 & 08:30 & $-205 B$ & $-183 \mathrm{~B}$ & $-192.75 B$ & $9.2 \mathrm{~B}$ \\
\hline Durable Goods Orders & $\mathrm{CB}$ & 12 & 08:30 & -0.0150 & 0.0150 & 0.0034 & 0.0111 \\
\hline Empire Manufacturing & FR & 12 & 08:30 & 1 & 25 & 15.983 & 6.1573 \\
\hline Employment Cost Index & BLS & 4 & 08:30 & 0.0080 & 0.0100 & 0.0085 & 0.0010 \\
\hline FOMC Rate Decision (Upper Bound) & FR & 8 & 14:15 & 0.0250 & 0.0425 & 0.0338 & 0.0061 \\
\hline Factory Orders & $\mathrm{CB}$ & 12 & 10:00 & -0.0230 & 0.0300 & 0.0058 & 0.0153 \\
\hline GDP Annualised QoQ & BEA & 12 & 08:30 & 0.0330 & 0.0430 & 0.0368 & 0.0029 \\
\hline Housing Starts & $\mathrm{CB}$ & 12 & 08:30 & $1903 \mathrm{~K}$ & $2090 \mathrm{~K}$ & $2013.6 \mathrm{~K}$ & $56.2 \mathrm{~K}$ \\
\hline ISM Manufacturing & ISM & 12 & 10:00 & 51.400 & 58.500 & 55.383 & 2.4778 \\
\hline Import Price Index MoM & BLS & 12 & 08:30 & -0.0050 & 0.0140 & 0.0047 & 0.0068 \\
\hline Industrial Production MoM & FR & 12 & 09:15 & -0.004 & 0.01 & 0.0034 & 0.0031 \\
\hline Initial Jobless Claims & DL & 52 & 08:30 & $309 \mathrm{~K}$ & $450 \mathrm{~K}$ & $329.8 \mathrm{~K}$ & $24.5 \mathrm{~K}$ \\
\hline Leading Index & $\mathrm{CB}$ & 12 & 10:00 & -0.0050 & 0.0080 & 0.0003 & 0.0040 \\
\hline Monthly Budget Statement & $\mathrm{T}$ & 12 & 14:00 & $-100 \mathrm{~B}$ & $60 \mathrm{~B}$ & $-26.9 \mathrm{~B}$ & $50.8 \mathrm{~B}$ \\
\hline NAHB Housing Market Index & NAHB & 12 & 13:00 & 61 & 71 & 67.833 & 2.9797 \\
\hline New Home Sales & $\mathrm{CB}$ & 12 & 10:00 & $1125 \mathrm{~K}$ & $1350 \mathrm{~K}$ & $1253.4 \mathrm{~K}$ & $77.5 \mathrm{~K}$ \\
\hline Nonfarm Productivity & BLS & 8 & 08:30 & 0.0150 & 0.0450 & 0.0238 & 0.0100 \\
\hline PCE Core YoY & BEA & 11 & 08:30 & 0.0150 & 0.0190 & 0.0174 & 0.0016 \\
\hline Personal Consumption & BEA & 10 & 08:30 & 0.0300 & 0.0430 & 0.0359 & 0.0042 \\
\hline Personal Income & BEA & 12 & 08:30 & -0.0260 & 0.0340 & 0.0041 & 0.0128 \\
\hline Personal Spending & BEA & 12 & 08:30 & -0.0020 & 0.0100 & 0.0045 & 0.0036 \\
\hline Philadelphia Fed Business Outlook & PFR & 12 & 12:00 & 10 & 25 & 14.733 & 4.6582 \\
\hline Retail Sales Advance MoM & $\mathrm{CB}$ & 12 & 08:30 & -0.0140 & 0.0210 & 0.0038 & 0.0093 \\
\hline Trade Balance & $\mathrm{CB}$ & 12 & 09:30 & $-62.9 \mathrm{~B}$ & $-54 \mathrm{~B}$ & $-58.7 \mathrm{~B}$ & $2.6 \mathrm{~B}$ \\
\hline Unemployment Rate & BLS & 12 & 08:30 & 0.0500 & 0.0540 & 0.0516 & 0.0015 \\
\hline Wholesale Inventories MoM & $\mathrm{CB}$ & 12 & 10:00 & 0.0030 & 0.0090 & 0.0056 & 0.0017 \\
\hline
\end{tabular}


TABle 2.2: Summary Statistics of Pre-scheduled Macroeconomic Announcements Survey (Canada).

This table provides summary statistics for macroeconomic announcements in the US and Canada in the year 2005. The columns present, in order: the type of macroeconomic announcement in Canada, the source of announcement releases (the abbreviation of each source is defined below), the number of observations of each announcement, the time of the announcement, minimum, maximum, mean, and standard deviation of observations in each announcement.

Abbreviations: BC: Bank of Canada, STCA: Statistics Canada, K: Thousands, B: Billion.

\begin{tabular}{llllllll}
\hline Event & Source & Obs. & Time & Min & Max & Mean & Std. \\
\hline Canadian Announcements & & & & & & & \\
Bank of Canada Rate Decision & BC & 8 & $09: 00$ & 0.0250 & 0.0325 & 0.0269 & 0.0029 \\
Building Permits MoM & STCA & 12 & $08: 30$ & -0.0500 & 0.0400 & -0.0082 & 0.0270 \\
CPI YoY & STCA & 12 & $07: 00$ & 0.0180 & 0.0330 & 0.0233 & 0.0046 \\
Capacity Utilization Rate & STCA & 4 & $08: 30$ & 0.8560 & 0.8720 & 0.8625 & 0.0072 \\
Current Account Balance & STCA & 4 & $08: 30$ & $3.4 \mathrm{~B}$ & $9 \mathrm{~B}$ & $6.14 \mathrm{~B}$ & $2.51 \mathrm{~B}$ \\
Housing Starts & STCA & 12 & $08: 30$ & $215 \mathrm{~K}$ & $230 \mathrm{~K}$ & $224.5 \mathrm{~K}$ & $4.48 \mathrm{~K}$ \\
Industrial Product Price MoM & STCA & 12 & $08: 30$ & -0.0100 & 0.0090 & 0.0030 & 0.0047 \\
Int'l Merchandise Trade & STCA & 12 & $08: 30$ & $4.3 \mathrm{~B}$ & $6.9 \mathrm{~B}$ & $5.19 \mathrm{~B}$ & $0.787 \mathrm{~B}$ \\
Labor Productivity QoQ & STCA & 4 & $08: 30$ & 0.0010 & 0.0050 & 0.0030 & 0.0016 \\
Manufacturing Sales MoM & STCA & 12 & $08: 30$ & -0.0060 & 0.0100 & 0.0038 & 0.0046 \\
New Housing Price Index MoM & STCA & 12 & $08: 30$ & 0.0030 & 0.0050 & 0.0039 & 0.0007 \\
Quarterly GDP Annualised & STCA & 4 & $08: 30$ & 0.0170 & 0.0360 & 0.0263 & 0.0078 \\
Raw Materials Price Index MoM & STCA & 12 & $08: 30$ & -0.0200 & 0.0400 & 0.0148 & 0.0201 \\
Retail Sales MoM & STCA & 12 & $08: 30$ & -0.0050 & 0.0140 & 0.0031 & 0.0065 \\
Unemployment Rate & STCA & 12 & $07: 00$ & 0.0660 & 0.0730 & 0.0687 & 0.0019 \\
Wholesale Trade Sales MoM & STCA & 12 & $08: 30$ & 0.0020 & 0.0070 & 0.0050 & 0.0015 \\
\hline
\end{tabular}


announcements into three samples, based on the relevance value that is reported by Bloomberg. The first sample includes the Full set of announcements in the US and Canada. The second sample restricts attention to the Relatively Important announcements: change in non-farm payrolls, initial jobless claims, FOMC rate decisions, annualised GDP Quarter-on-Quarter (QoQ), ISM manufacturing, CPI Month-on-Month (MoM), the conference board consumer confidence, durable goods orders, retail sales advance MoM, new home sales, housing starts, industrial production MoM, unemployment rate, factory orders, personal income, and personal spending, in the US. For Canada, the relatively important announcements are bank of Canada rate decisions, CPI Year-on-Year (YoY), annualised quarterly GDP, and the unemployment rate in Canada. Finally, the third sample restricts the announcements even further to consider only the Most Important announcements: change in non-farm payrolls, initial jobless claims, FOMC rate decisions (upper bound), annualised GDP QoQ, ISM manufacturing, and CPI MoM in the US as well as bank of Canada rate decisions and CPI YoY in Canada. In the robustness section, I separate the announcements into four samples based on their economic function: output, prices, employment, and rate decision.

\subsubsection{Constructing Variables}

In this section, I describe the construction of variables for my study. The variables of interest include realised variance as the dependent variable, along with a number of explanatory variables.

\section{Realised Variance}

Conventional GARCH models (as a parametric measure of volatility) are unable to explain jumps in volatility, and the low autocorrelation in squared 
returns when evaluated out of sample (Kliber and Bedowska-Sojka, 2010). However, I can use the aggregated high frequency returns, which are an observable estimate of latent volatility and a good estimate from which to forecast future volatility. The dependent variable in my model is realised variance $(R V)$. I examine Realised Variance in the 15-minute interval before and after the 8:30. I choose the 8:30 announcement instead of other announcements because a large portion of important news will come into the market at this time, and by focusing on announcements at the same time of day, it is robust to intraday patterns in volatility responses. However, in Section 2.4.6, I analyse the impact of news at times other than 8:30. I apply the Hansen and Lunde (2006) method to measure the realised variance, which is the sum of squares of the difference in log mid-quote prices in each one-minute interval during a 15-minute interval:

$$
R V_{t}=\sum_{\tau=1}^{15} r_{t-\tau}^{2}=\sum_{\tau=1}^{15}\left(\log \left(m q_{t-\tau}\right)-\log \left(m q_{t-\tau-1}\right)\right)^{2} * 1000
$$

where $R V_{t}$ is the realised variance during each 15-minute interval, $r_{\tau}$ is the return over each 1-minute interval, $m q_{\tau}$ is the mid-quote (average of the best bid and best ask at the end of each one-minute interval), $\tau$ is the counter of each one-minute interval, and 1000 is a rescaling for comparability with other variables. If there is an interval without any reported quote, I use the quote from the previous interval. Choosing the one-minute interval is ad hoc and changing it does not affect my results.

In traditional realised variance calculations, the limit order book is not available, and the returns used to calculate realised variance are based on transaction prices. However, in this study, I do not need to rely on transaction prices since I have access to the limit order book with detailed information. In this regard, I model realised variance as an ex-post and observable volatility in the FX market using the limit order book.

In the robustness section, I apply two alternative measures to see 
whether the results change. The first alternative to realised variance is absolute return. Many scholars have used the absolute return as a proxy for volatility in the literature (see Jones, Kaul, and Lipson (1994), Andersen and Bollerslev (1997, 1998), Granger and Sin (2000), Ahn, Bae, and K. Chan (2001), Forsberg and Ghysels (2007), Foucault, Moinas, and Theissen (2007)). I define absolute return as:

$$
\left|R_{t}\right|=\left|\log \left(m q_{t-1}\right)-\log \left(m q_{t-16}\right)\right| * 1000,
$$

where $\left|R_{t}\right|$ is the absolute return for each 15-minute interval, and $m q_{t}$ is the average of the best bid and the best ask at time $t .{ }^{1}$

In high frequency data, there is a possibility that mid-quotes differ from their true value due to market microstructure effects (Hasbrouck, 1993). Therefore, I propose a third measure that takes into consideration the possibility of negative correlation of returns inducing predictability in future returns. The third measure of volatility is the modified absolute return. I compute the 15-minute interval absolute returns during the active time of the trading day (6:00 a.m - 5:00 p.m). Then I regress the $A R$ values on their lagged values and retain the absolute values of residuals of this regression as my third proxy for volatility. This measure is used by Forsberg and Ghysels (2007) and Foucault, Moinas, and Theissen (2007). I define the third measure $R^{r e s i}$ as:

$$
\begin{gathered}
\left|R_{t}\right|=\beta_{0}+\beta_{1}\left|R_{t-15}\right|+\epsilon_{t} \\
R_{t}^{r e s i}=\left|\epsilon_{t}\right| * 1000,
\end{gathered}
$$

where $\left|R_{t}\right|$ is the absolute return for each 15-minute interval, $\left|R_{t-15}\right|$ is the 15-minute lag absolute return, $\epsilon_{t}$ is the regression residual, and $R_{t}^{r e s i}$ is the time series of modified absolute returns.

\footnotetext{
${ }^{1}$ In all my measures, I use the quote from the preceding time period because the effect of each quote will be revealed in the subsequent time period.
} 


\section{Explanatory Variables}

A key explanatory variable in my model set up is the existence of news in the market. I define a dummy variable, which is equal to one if there is news and equal to zero if there is not any news. This measure examines the presence of news and its effect on the market. Another explanatory variable is the amount of surprise or shock in the market. This can be calculated by finding the difference between the realised value and analyst forecast, divided by the standard deviation of each sample. As a result, in this process, all the macroeconomic variables with different measurement units will be standardised, since different units of measurement will shift both means and standard deviations.

$$
\operatorname{Sur}_{k, t}=\left|\frac{\text { Actual }_{k, t}-\text { Survey }_{k, t}}{\text { S.d }\left(\text { Actual }_{k}-\text { Survey }_{k}\right)}\right|,
$$

where Actual $_{k, t}$ is the realised value of announcement $k$ at time $t$, Survey ${ }_{k, t}$ is the analyst forecasts of announcement $k$ at time $t$, and $S . d$ is the standard deviation of the difference in the numerator. Many scholars have exploited this method to measure public information shocks in financial markets (see Balduzzi, Elton, and Green (2001), Pasquariello and Vega (2007), and Ben-Omrane and Hafner (2015)). I will examine the surprise with the positive and negative sign as well as zero surprise in the robustness section (in the literature there are studies that evaluate the sign of the surprise: see Gosnell, Keown, and Pinkerton (1996), and Andersen, Bollerslev, Diebold, and Vega (2003)).

Among the other explanatory variables, the bid-ask spread variable plays an important role in the literature and many scholars use this proxy to explain market conditions. Brekke and Oksendal (1988) consider the bid-ask spread as a signal of liquidity and transaction costs in the market. They argue that the inventory management cost for market makers decreases when many uninformed traders are present in the market. This 
situation results in a lower level of adverse selection, and consequently a narrower bid-ask spread. In my analyses, the spread is an explanatory variable, being measured as the difference between the best ask quote and the best bid quote at the end of each 15-minute interval. It is defined as:

$$
S_{t}=a p_{0, t-1}-b p_{0, t-1}
$$

where $a p_{0, t-1}$ is the best ask quote one minute before $t$, and $b p_{0, t-1}$ is the best bid quote one minute before $t$, both taken from the electronic order book. I use this variable as a liquidity measure to explain realised variance in the market. Pasquariello and Vega (2007) use the change in bid-ask spread to examine if anonymity matters in an electronic limit order book. However, I use this measure to capture the sensitivity of realised variance, which counts as a private information shock.

The explanatory variables $D_{0, t}$ and $D_{1, t}$ are measured as the summation of quantity quoted at the best quote and the second best quote at the end of each 15-minute interval respectively:

$$
\begin{aligned}
& D_{0, t}=a q_{0, t-1}+b q_{0, t-1} \\
& D_{1, t}=a q_{1, t-1}+b q_{1, t-1},
\end{aligned}
$$

where $D_{0, t}$ is the depth at the best quote; $D_{1, t}$ is the depth at the second best quote; $a q_{0, t-1}$ is the quantity at the best ask price one minute before $t ; b q_{0, t-1}$ is the quantity at the best bid price one minute before $t ; a q_{1, t-1}$ is the quantity at the second best ask price one minute before $t$; and $b q_{1, t-1}$ is the quantity at the second best bid price one minute before $t$. Depth at the best quote is an active and aggressive quote and has a high likelihood of getting exercised. However, in a market with asymmetric information, traders hide their quotes behind the best quote to avoid this probability due to uncertainty (Jiang, Lo, and Valente, 2014). As a result, I expect that a decline in liquidity will have a negative impact on the depth at the 
best quote and a positive impact on the depth behind the best quote. The uncertainty among traders that is caused by having different levels of information leads to an illiquid market when they quote further away from the best quotes. The primary assumption here is that traders are waiting for some information to be released by scheduled announcements. In this regard, there is an uncertain situation for traders but the level of uncertainty varies among traders. Some of the traders are more expert and have better understanding of the market whereas some of traders are not as experienced.

Recent studies suggest that order flow matters in determining exchange rates and in explaining fluctuations in the market. Evans and Lyons (2002) state that order flow is a conduit for aggregating heterogeneous expectations about the future state of the economy and different interpretations of news in real time. The order flow will be driven by a broad set of macroeconomic information, which is the concern of exchange rate theories (Rime, Sarno, and Sojli, 2010). In line with the literature, I include order flow as an explanatory variable in my model to explain realised variance in the market. The value of order flow is the difference between the buy volume and the sell volume during the 15-minute interval. I use the absolute value of order flow as:

$$
\left|A O F_{t}\right|=\sum_{\tau=1}^{15}\left|Q_{t-\tau-1, t-\tau}^{A s k}-Q_{t-\tau-1, t-\tau}^{B i d}\right|,
$$

where $\left|A O F_{t}\right|$ is the absolute order flow of each 15-minute interval, $Q_{t-\tau-1, t-\tau}^{A s k}$ is the quantity quoted on the ask side between times $t-\tau-1$ and $t-\tau$, and $Q_{t-\tau-1, t-\tau}^{B i d}$ is the quantity quoted in the bid side between times $t-\tau-1$ and $t-\tau$.

Finally, the last explanatory variable is order imbalance, which is informative about price movements. Order imbalance has a significant effect on price and liquidity for two reasons. Firstly, in line with Kyle's theory 
of price information, order imbalance is a proxy for private information that can decrease the liquidity in the market temporarily (and in an extreme case, permanently). Secondly, the inventory problem faced by the market maker, which is adjusting the bid-ask spreads according to inventory, is exacerbated due to extreme order imbalances (Chordia, Roll, and Subrahmanyam, 2002). I measure the order imbalance by finding the difference between the bid-depth and ask-depth at the end of each 15-minute interval.

$$
\left|A O I_{t}\right|=\left|D_{e p t h}^{A s k}-D_{t-1}^{A e p t h} h_{t-1}^{\text {Bid }}\right|,
$$

where $\left|A O I_{t}\right|$ is the absolute order imbalance of each 15-minute interval, $D e p t h_{t-1}^{A s k}$ is the ask depth at one minute before $t$, and Dept $h_{t-1}^{B i d}$ is the bid depth at one minute before $t$. In the following section, I use all these measurements in a regression model to explain the realised variance in the Canadian FX market.

\subsection{Empirical Analysis}

In this section, I evaluate the impact of 8:30 macroeconomic announcements on realised variance. I evaluate Realised Variance changes on news days and no-news days as well as before and after the announcements. I set up my main regression model, and evaluate the relationship and sensitivity of Realised Variance to my explanatory variables.

\subsubsection{The Model}

I split each trading day into two 15-minute intervals. The first interval examines market conditions before 8:30, and the second interval examines market conditions after 8:30. This process is applied for both news days and no-news days. 
TABle 2.3: Difference in Difference t-test Results for News Days vs No-news Days and before vs after Announcements.

This table presents the mean of realised variance before and after 8:30, news days and no-news days, and in three samples: full sample, relatively important, and the most important. The $t$-test evaluates whether there is a significant difference between news days and no-news days as well as before and after announcements separately. The number of observations for Full sample, relatively important (RI) sample, and most important (MI) sample are 330, 240, and 176 respectively. I use UNIVARIATE procedure in SAS to test if my samples before and after 8:30 are random with normal distribution. Results show that all three samples before and after 8:30 have normal distribution.

Note: The superscripts ${ }^{* * *}, * *$, and $*$ indicate significance at the $1 \%, 5 \%$, and $10 \%$ level respectively.

\begin{tabular}{|c|c|c|c|}
\hline Realised Variance & Before & After & After-Before \\
\hline \multicolumn{4}{|c|}{ Panel A: Full Sample News (Full) } \\
\hline News & 0.1103 & 0.2920 & $\begin{array}{l}0.1817^{* * *} \\
(0.0292)\end{array}$ \\
\hline No News & 0.0934 & 0.1031 & $\begin{array}{c}0.0097 \\
(0.3850)\end{array}$ \\
\hline Diff(News and No-News) & $\begin{array}{c}0.0169 \\
(0.0153)\end{array}$ & $\begin{array}{l}0.1889^{* * *} \\
(0.0498)\end{array}$ & $\begin{array}{l}0.1720^{* * *} \\
(0.0518)\end{array}$ \\
\hline \multicolumn{4}{|c|}{ Panel B: Relatively Important News (RI) } \\
\hline News & 0.1107 & 0.2807 & $\begin{array}{l}0.1700^{* * *} \\
(0.0321)\end{array}$ \\
\hline No News & 0.0973 & 0.1031 & $\begin{array}{c}0.0058 \\
(0.6010)\end{array}$ \\
\hline Diff(News and No-News) & $\begin{array}{c}0.0134 \\
(0.0161) \\
\end{array}$ & $\begin{array}{l}0.1776^{* * *} \\
(0.0442)\end{array}$ & $\begin{array}{l}0.1642^{* * *} \\
(0.0467)\end{array}$ \\
\hline \multicolumn{4}{|c|}{ Panel C: Most Important News (MI) } \\
\hline News & 0.0883 & 0.4590 & $\begin{array}{l}0.3707^{* * *} \\
(0.0891)\end{array}$ \\
\hline No News & 0.0973 & 0.1031 & $\begin{array}{c}0.0058 \\
(0.6010)\end{array}$ \\
\hline Diff(News and No-News) & $\begin{array}{c}-0.0090 \\
(0.0189)\end{array}$ & $\begin{array}{l}0.3559^{* * *} \\
(0.0661)\end{array}$ & $\begin{array}{l}0.3649^{* * *} \\
(0.0676)\end{array}$ \\
\hline
\end{tabular}


Table 2.3 presents the results. I test the hypothesis of no effect from news for all trading days based on three different samples of announcements. The first sample includes the Full set of pre-scheduled macroeconomic announcements; the second sample includes the Relatively Important pre-scheduled macroeconomic announcements; and finally, the last sample includes the Most Important pre-scheduled macroeconomic announcements. I test Realised Variance changes between news days and no-news days, as well as before and after the announcements on news days to see if they are significant or not.

Table 2.3 shows that after 8:30, there is a significant difference in realised variance. This conclusion holds for all three samples of announcements. The difference is much higher for the Most Important news sample compared with the other two samples. In addition, the difference between news days and no-news days is insignificant before 8:30, while this difference is higher and significant after 8:30. The difference in difference between news days and no-news days is significant for all three samples. My result for the Most Important sample is much higher compared with the other two samples. These volatility increases, however, could be due to either public information shocks or private information shocks.

\subsubsection{Regression Analysis}

In this section, my goal is to evaluate the impact of public information shocks and private information shocks by setting up a regression analysis. Before setting up the regression model, I check the sample data before and after 8:30 to see if they are stationary. Table 2.4 shows the unit root test for realised variance before and after 8:30. Results imply that there is no evidence of a unit root and both samples before and after the 8:30 are stationary and they do not have a trend as well.

I aim to break down the total effect of the arrival of macroeconomic 
TABLE 2.4: Realised Variance Unit Root Test Before and After 8:30

This table presents unit root tests to find if the samples before and after 8:30 are stationary. There are three different types of test to check the stationary. Results show that the samples are stationary without any trend and need of having difference. Note that the ADF test in SAS presents three tests: Rho, Tau, and F tests.

\begin{tabular}{|c|c|c|c|c|c|c|c|}
\hline \multicolumn{8}{|c|}{ Augmented Dickey-Fuller Unit Root Tests } \\
\hline & & & & Before & & & \\
\hline \multirow[t]{2}{*}{ Type } & Lags & Rho & $\operatorname{Pr}<$ Rho & Tau & $\operatorname{Pr}<$ Tau & $\mathrm{F}$ & $\operatorname{Pr}>F$ \\
\hline & 0 & -58.49 & $<.00$ & -6.06 & $<.00$ & & \\
\hline \multirow{3}{*}{ Zero Mean } & 1 & -24.66 & 0.00 & -3.54 & 0.00 & & \\
\hline & 2 & -17.54 & 0.00 & -2.95 & 0.00 & & \\
\hline & 0 & -152.51 & 0.00 & -12.22 & $<.00$ & 74.64 & 0.00 \\
\hline \multirow[t]{3}{*}{ Single Mean } & 1 & -135.04 & 0.00 & -8.14 & $<.000$ & 33.16 & 0.00 \\
\hline & 2 & -185.11 & 0.00 & -7.54 & $<.00$ & 28.44 & 0.00 \\
\hline & 0 & -153.16 & 0.00 & -12.22 & $<.00$ & 74.66 & 0.00 \\
\hline \multirow{3}{*}{ Trend } & 1 & -136.45 & 0.00 & -8.16 & $<.00$ & 33.28 & 0.00 \\
\hline & 2 & -188.22 & 0.00 & -7.55 & $<.00$ & 28.50 & 0.00 \\
\hline & \multicolumn{7}{|c|}{ After } \\
\hline \multirow[t]{2}{*}{ Type } & Lags & Rho & $\operatorname{Pr}<$ Rho & Tau & $\operatorname{Pr}<$ Tau & $\mathrm{F}$ & $\operatorname{Pr}>F$ \\
\hline & 0 & -98.08 & $<.00$ & -8.70 & $<.00$ & & \\
\hline \multirow[t]{3}{*}{ Zero Mean } & 1 & -44.65 & $<.00$ & -4.75 & $<.00$ & & \\
\hline & 2 & -30.36 & $<.00$ & -3.71 & 0.00 & & \\
\hline & 0 & -164.18 & 0.00 & -13.45 & $<.00$ & 90.48 & 0.00 \\
\hline \multirow[t]{3}{*}{ Single Mean } & 1 & -137.58 & 0.00 & -8.28 & $<.00$ & 34.28 & 0.00 \\
\hline & 2 & -158.37 & 0.00 & -7.18 & $<.00$ & 25.76 & 0.00 \\
\hline & 0 & -166.14 & 0.00 & -13.54 & $<.00$ & 91.72 & 0.00 \\
\hline \multirow{2}{*}{ Trend } & 1 & -142.87 & 0.00 & -8.39 & $<.00$ & 35.21 & 0.00 \\
\hline & 2 & -171.04 & 0.00 & -7.32 & $<.00$ & 26.81 & 0.00 \\
\hline
\end{tabular}


announcements into public and private by setting up a regression model. In this regard, I define dummy variables to captures the effect of the existence/absence of news and before/after the news. A group of liquidity variables (i.e. spread, depth at the best quote, depth at the second best quote, absolute order flow, and absolute order imbalance) capture the effect of private information shocks whereas surprise is the proxy for capturing the impact of public information shocks. The model is set up as:

$$
\begin{aligned}
R V_{t} & =\beta_{0}+\beta_{1} \mathbf{1}_{\text {News }}+\beta_{2} \mathbf{1}_{\text {Post }}+\beta_{3} \mathbf{1}_{\text {News }} \mathbf{1}_{\text {Post }}+\theta_{1} R V_{t-15}+\theta_{2} R V_{t-15} \mathbf{1}_{\text {News }} \\
& +\theta_{3} R V_{t-15} \mathbf{1}_{\text {Post }}+\theta_{4} R V_{t-15} \mathbf{1}_{\text {News }} \mathbf{1}_{\text {Post }}+\delta_{1} X_{t}+\delta_{2} X_{t} \mathbf{1}_{\text {News }}+\delta_{3} X_{t} \mathbf{1}_{\text {Post }} \\
& +\delta_{4} X_{t} \mathbf{1}_{\text {News }} \mathbf{1}_{\text {Post }}+\gamma \operatorname{Sur}_{t} \mathbf{1}_{\text {Post }}+\epsilon_{t},
\end{aligned}
$$

where $R V_{t}$ is the realised variance; $\mathbf{1}_{\text {News }}$ is the news dummy equal to 1 or 0 in the event of news and no-news respectively; $\mathbf{1}_{\text {Post }}$ indicates that I evaluate the impact of explanatory variables after $8: 30 ; R V_{t-15}$ is the 15-minute lag realised variance; $X_{t}$ denotes a liquidity measure, which could be spread at the best quote $\left(S_{0}\right)$, depth at the best quote $\left(D_{0}\right)$, depth at the second best quote $\left(D_{1}\right)$, absolute order flow $(A O F)$, or absolute order imbalance $(A O I)$, and captures the sensitivity of Realised Variance to liquidity changes. Surprise (Sur), as a proxy for public information shocks, captures the impact of realised variance after the arrival of prescheduled macroeconomic announcements. The interaction of the news dummy, $\mathbf{1}_{\text {News }}$, and post 8:30 dummy, $\mathbf{1}_{\text {Post }}$, represents the difference-indifference impact that is the main focus of my analysis. In other words, this explanatory variable evaluates the sensitivity difference between news days and no-news days along with before and after the announcements. If $\delta_{4}>0$, then sensitivity of volatility to the microstructure variable rises after news announcements, and the private information effect is present. Conversely, if $\gamma>0$, then Realised Variance increases from surprises, consistent with public information shocks being present. Tables 2.5, 2.6, and 2.7 report my results of the regression model for each liquidity measure 
using the Full, Relatively Important, and Most Important samples. I examine the impact of all liquidity measures jointly in Section 2.4.5. Newey and West (1987) standard errors are applied to take care of autocorrelation and heteroskedasticity in the time series regression model.

Table 2.5 indicates that prior to the announcements, there are no significant differences between news days and no-news days for the Full sample. I capture this effect with the Dummy variable $\mathbf{1}_{\text {News }}$ and its interactions with $R V_{t-15}$ and $X_{t}$. Similarly, there are no significant results after 8:30 on no-news days. I capture this effect with the Dummy variable $\mathbf{1}_{\text {Post }}$ and its interactions with $R V_{t-15}$ and $X_{t}$. The sensitivity of realised variance to surprise as a proxy for public information shocks is almost zero $(\gamma \simeq 0)$. However, spread sensitivity (as a proxy for private information) has a significant increase with the arrival of macroeconomic announcements, and this is consistent with the existence of private information. In other words, realised variance is highly sensitive to bid-ask spread, as a proxy for private information shocks, following the arrival of news. A high level of information asymmetry, due to the new information, increases the risk of trading in the market. Information asymmetry results in adverse selection, and, in turn, dealers place their quotes carefully due to lack of enough information. Informed traders send their quotes with enough confident whereas uninformed traders are not sure about the right price or volume quotes. This finding, in the first place, is consistent with the literature that pre-scheduled macroeconomic announcements have a significant impact on realised variance due to repositioning and repricing by the traders in the market (see Andersen and Bollerslev (1997), Fleming and Remolona (1999), Balduzzi, Elton, and Green (2001), and Evans and Lyons (2008)). However, my results also imply that private information shocks are the key driving force as opposed to public information shocks.

I evaluate the same regression model for two alternative samples, Relatively Important (Table 2.6) and Most Important (Table 2.7). The results 
TABLE 2.5: Realised Variance at 8:30 Announcements Using the Full Sample.

Results of regression of dependent variable $(R V)$ realised variance on liquidity measures: $S_{0}$ is spread, $D_{0}$ is the depth at the best quote, $D_{1}$ is the depth at the second best quote, AOF is the absolute order flow, AOI is the absolute order imbalance. Descriptions of the calculation of the variables can be found in Section 2.3. There are 330 observations in full announcements sample. The superscripts ${ }^{* * *},{ }^{* *}$, and * indicate significance at the 1\%,5\%, and $10 \%$ level respectively. The significance is assessed using Newey-West standard errors.

\begin{tabular}{|c|c|c|c|c|c|}
\hline \multirow{2}{*}{ Variables } & \multicolumn{5}{|c|}{ Liquidity Measure (Full Announcements Sample) } \\
\hline & $\mathbf{S}_{0}$ & $\mathbf{D}_{0}$ & $\mathbf{D}_{1}$ & AOF & AOI \\
\hline Intercept & $\begin{array}{l}0.0642^{* * *} \\
(0.0181)\end{array}$ & $\begin{array}{l}0.0775^{* * *} \\
(0.0184)\end{array}$ & $\begin{array}{l}0.0786^{* * *} \\
(0.0176)\end{array}$ & $\begin{array}{l}0.0687^{* * *} \\
(0.0146)\end{array}$ & $\begin{array}{l}0.0681^{* * *} \\
(0.0136)\end{array}$ \\
\hline $\mathbf{1}_{\text {News }}$ & $\begin{array}{c}0.0397 \\
(0.0301)\end{array}$ & $\begin{array}{c}0.0239 \\
(0.0241)\end{array}$ & $\begin{array}{c}0.0158 \\
(0.0266)\end{array}$ & $\begin{array}{c}0.0256 \\
(0.0205)\end{array}$ & $\begin{array}{c}0.0325 \\
(0.0204)\end{array}$ \\
\hline $\mathbf{1}_{\text {Post }}$ & $\begin{array}{c}-0.0317 \\
(0.0272)\end{array}$ & $\begin{array}{c}-0.0201 \\
(0.0233)\end{array}$ & $\begin{array}{c}-0.0253 \\
(0.0250)\end{array}$ & $\begin{array}{c}-0.0181 \\
(0.0190)\end{array}$ & $\begin{array}{c}-0.0134 \\
(0.0187)\end{array}$ \\
\hline $\mathbf{1}_{\text {News }} \mathbf{1}_{\text {Post }}$ & $\begin{array}{c}0.0397 \\
(0.0577)\end{array}$ & $\begin{array}{c}0.1464^{* *} \\
(0.0729)\end{array}$ & $\begin{array}{c}0.1110 \\
(0.0743)\end{array}$ & $\begin{array}{l}0.1235^{* *} \\
(0.0614)\end{array}$ & $\begin{array}{l}0.1236^{* *} \\
(0.0646)\end{array}$ \\
\hline $\mathrm{RV}_{t-15}$ & $\begin{array}{l}0.3821^{* * *} \\
(0.0405)\end{array}$ & $\begin{array}{l}0.3826^{* * *} \\
(0.0395)\end{array}$ & $\begin{array}{l}0.3810^{* * *} \\
(0.0406)\end{array}$ & $\begin{array}{l}0.3807^{* * *} \\
(0.0422)\end{array}$ & $\begin{array}{l}0.3847^{* * *} \\
(0.0393)\end{array}$ \\
\hline $\mathrm{RV}_{t-15} \mathbf{1}_{\text {News }}$ & $\begin{array}{c}-0.1217 \\
(0.1655)\end{array}$ & $\begin{array}{c}-0.1255 \\
(0.1637)\end{array}$ & $\begin{array}{c}-0.1211 \\
(0.1651)\end{array}$ & $\begin{array}{c}-0.1510 \\
(0.1729)\end{array}$ & $\begin{array}{c}-0.1353 \\
(0.1630)\end{array}$ \\
\hline $\mathrm{RV}_{t-15} \mathbf{1}_{\text {Post }}$ & $\begin{array}{c}0.1443 \\
(0.0985)\end{array}$ & $\begin{array}{c}0.1695 \\
(0.1108)\end{array}$ & $\begin{array}{c}0.1452 \\
(0.1008)\end{array}$ & $\begin{array}{c}0.0953 \\
(0.1676)\end{array}$ & $\begin{array}{c}0.1745 \\
(0.1118)\end{array}$ \\
\hline $\mathrm{RV}_{t-15} \mathbf{1}_{\text {News }} \mathbf{1}_{\text {Post }}$ & $\begin{array}{c}0.1455 \\
(0.3381)\end{array}$ & $\begin{array}{c}0.2825 \\
(0.3555)\end{array}$ & $\begin{array}{c}0.1854 \\
(0.3764)\end{array}$ & $\begin{array}{c}0.2922 \\
(0.4017)\end{array}$ & $\begin{array}{c}0.2638 \\
(0.3472)\end{array}$ \\
\hline $\mathrm{X}_{t}$ & $\begin{array}{c}0.0095 \\
(0.0429)\end{array}$ & $\begin{array}{c}-0.0018 \\
(0.0015)\end{array}$ & $\begin{array}{c}-0.0017 \\
(0.0011)\end{array}$ & $\begin{array}{c}0.0000 \\
(0.0002)\end{array}$ & $\begin{array}{c}-0.0006 \\
(0.0016)\end{array}$ \\
\hline $\mathrm{X}_{t} \mathbf{1}_{\text {News }}$ & $\begin{array}{c}-0.0464 \\
(0.0704)\end{array}$ & $\begin{array}{c}0.0005 \\
(0.0021)\end{array}$ & $\begin{array}{c}0.0016 \\
(0.0020)\end{array}$ & $\begin{array}{c}0.0001 \\
(0.0002)\end{array}$ & $\begin{array}{c}-0.0020 \\
(0.0030)\end{array}$ \\
\hline $\mathrm{X}_{t} \mathbf{1}_{\text {Post }}$ & $\begin{array}{c}0.0612 \\
(0.0700)\end{array}$ & $\begin{array}{c}0.0005 \\
(0.0018)\end{array}$ & $\begin{array}{c}0.0014 \\
(0.0023)\end{array}$ & $\begin{array}{c}0.0002 \\
(0.0004)\end{array}$ & $\begin{array}{c}-0.0015 \\
(0.0022)\end{array}$ \\
\hline $\mathrm{X}_{t} \mathbf{1}_{\text {News }} \mathbf{1}_{\text {Post }}$ & $\begin{array}{l}0.2337^{* *} \\
(0.1081)\end{array}$ & $\begin{array}{c}-0.0059 \\
(0.0059)\end{array}$ & $\begin{array}{c}0.0015 \\
(0.0066)\end{array}$ & $\begin{array}{c}-0.0004 \\
(0.0005)\end{array}$ & $\begin{array}{c}-0.0030 \\
(0.0068)\end{array}$ \\
\hline $\operatorname{Sur}_{t} \mathbf{1}_{\text {Post }}$ & $\begin{array}{c}-0.0039 \\
(0.0208)\end{array}$ & $\begin{array}{c}0.0106 \\
(0.0247)\end{array}$ & $\begin{array}{c}0.0132 \\
(0.0253)\end{array}$ & $\begin{array}{c}0.0165 \\
(0.0271)\end{array}$ & $\begin{array}{c}0.0108 \\
(0.0251)\end{array}$ \\
\hline Adjusted $\mathrm{R}^{2}$ & 0.2930 & 0.1630 & 0.1561 & 0.1527 & 0.1628 \\
\hline
\end{tabular}


are insignificant for both samples, Relatively Important and Most Important. This is consistent with the conjecture that there is extensive public analysis about the Relatively Important and Most Important announcements, which decreases the value of private analysis. To summarise, bid-ask spread sensitivity as a proxy for private information shocks shows a significant relationship with realised variance at the $5 \%$ level in the Full sample. However, the bid-ask spread coefficients for the Relatively Important and Most Important samples are insignificant. Liquidity variables are a sign of existing private information in the market that results in a more volatile market. Uninformed traders are exposed to financial losses in this market and they are better off to avoid trading by quoting further away from the best quote or exiting from the market.

\subsection{Robustness and Extensions}

In Section 2.3, I apply the Hansen and Lunde (2006) method to measure realised variance in the Canadian FX market. The results indicate that the arrival of new information has a significant impact on realised variance primarily due to private information shocks. Public information shocks have an insignificant impact. Spread is the key variable for measuring private information shocks. Other liquidity measures in the model do not produce significant results. In the following, I examine more tests to find if there is any change in my results. The robustness tests consist of: evaluating realised variance by removing the lag from the regression model, testing realised variance in the US and Canada separately, more tests on the surprise variable, alternative measures of volatility, the effect of all liquidity variables jointly, the effect of news at times other than at 8:30, the magnitude of liquidity variable changes, evaluation in economic samples, and finally a summary of all cases. 
TABLE 2.6: Realised Variance at 8:30 Announcements Using the RI Sample.

Results of regression of dependent variable $(R V)$ realised variance on liquidity measure: $S_{0}$ is spread, $D_{0}$ is the depth at the best quote, $D_{1}$ is the depth at the second best quote, AOF is the absolute order flow, AOI is the absolute order imbalance. Descriptions of the calculation of the variables can be found in Section 2.3. There are 240 observations in RI announcements sample. The superscripts ${ }^{* * *},{ }^{* *}$, and ${ }^{*}$ indicate significance at the $1 \%, 5 \%$, and $10 \%$ level respectively. The significance is assessed using Newey-West standard errors.

\begin{tabular}{|c|c|c|c|c|c|}
\hline \multirow{2}{*}{ Variables } & \multicolumn{5}{|c|}{ Liquidity Measure (RI Announcements Sample) } \\
\hline & $\mathbf{S}_{0}$ & $\mathbf{D}_{0}$ & $\mathbf{D}_{1}$ & AOF & AOI \\
\hline Intercept & $\begin{array}{l}0.0642^{* * *} \\
(0.0183)\end{array}$ & $\begin{array}{l}0.0775^{* * *} \\
(0.0185)\end{array}$ & $\begin{array}{l}0.0786^{* * *} \\
(0.0178)\end{array}$ & $\begin{array}{l}0.0687^{* * *} \\
(0.0148)\end{array}$ & $\begin{array}{l}0.0681^{* * *} \\
(0.0137)\end{array}$ \\
\hline $\mathbf{1}_{\text {News }}$ & $\begin{array}{c}0.0221 \\
(0.0308)\end{array}$ & $\begin{array}{c}0.0191 \\
(0.0258)\end{array}$ & $\begin{array}{c}0.0184 \\
(0.0288)\end{array}$ & $\begin{array}{c}0.0194 \\
(0.0226)\end{array}$ & $\begin{array}{c}0.0230 \\
(0.0213)\end{array}$ \\
\hline $\mathbf{1}_{\text {Post }}$ & $\begin{array}{c}-0.0317 \\
(0.0274)\end{array}$ & $\begin{array}{c}-0.0201 \\
(0.0234)\end{array}$ & $\begin{array}{c}-0.0253 \\
(0.0252)\end{array}$ & $\begin{array}{c}-0.0181 \\
(0.0191)\end{array}$ & $\begin{array}{c}-0.0134 \\
(0.0189)\end{array}$ \\
\hline $\mathbf{1}_{\text {News }} \mathbf{1}_{\text {Post }}$ & $\begin{array}{c}0.0504 \\
(0.0628)\end{array}$ & $\begin{array}{c}0.1098 \\
(0.0755)\end{array}$ & $\begin{array}{c}0.0657 \\
(0.0701)\end{array}$ & $\begin{array}{c}0.1067 \\
(0.0683)\end{array}$ & $\begin{array}{c}0.1126 \\
(0.0705)\end{array}$ \\
\hline $\mathrm{RV}_{t-15}$ & $\begin{array}{l}0.3821^{* * *} \\
(0.0408)\end{array}$ & $\begin{array}{l}0.3826^{* * *} \\
(0.0398)\end{array}$ & $\begin{array}{l}0.3810^{* * *} \\
(0.0409)\end{array}$ & $\begin{array}{l}0.3807^{* * *} \\
(0.0425)\end{array}$ & $\begin{array}{l}0.3847^{* * *} \\
(0.0396)\end{array}$ \\
\hline $\mathrm{RV}_{t-15} \mathbf{1}_{\text {News }}$ & $\begin{array}{c}0.0028 \\
(0.2098)\end{array}$ & $\begin{array}{c}-0.0008 \\
(0.2110)\end{array}$ & $\begin{array}{c}-0.0080 \\
(0.2156)\end{array}$ & $\begin{array}{r}-0.0140 \\
(0.2181)\end{array}$ & $\begin{array}{c}-0.0031 \\
(0.2105)\end{array}$ \\
\hline $\mathrm{RV}_{t-15} \mathbf{1}_{\text {Post }}$ & $\begin{array}{c}0.1443 \\
(0.0992)\end{array}$ & $\begin{array}{c}0.1695 \\
(0.1117)\end{array}$ & $\begin{array}{c}0.1452 \\
(0.1015)\end{array}$ & $\begin{array}{c}0.0953 \\
(0.1688)\end{array}$ & $\begin{array}{c}0.1745 \\
(0.1127)\end{array}$ \\
\hline $\mathrm{RV}_{t-15} \mathbf{1}_{\text {News }} \mathbf{1}_{\text {Post }}$ & $\begin{array}{c}-0.1032 \\
(0.4005)\end{array}$ & $\begin{array}{c}-0.0755 \\
(0.4285)\end{array}$ & $\begin{array}{c}-0.2461 \\
(0.4852)\end{array}$ & $\begin{array}{c}0.0124 \\
(0.4602)\end{array}$ & $\begin{array}{c}-0.0339 \\
(0.4075)\end{array}$ \\
\hline $\mathrm{X}_{t}$ & $\begin{array}{c}0.0095 \\
(0.0432)\end{array}$ & $\begin{array}{c}-0.0018 \\
(0.0015)\end{array}$ & $\begin{array}{c}-0.0017 \\
(0.0011)\end{array}$ & $\begin{array}{c}0.0000 \\
(0.0002)\end{array}$ & $\begin{array}{c}-0.0006 \\
(0.0016)\end{array}$ \\
\hline $\mathrm{X}_{t} \mathbf{1}_{\text {News }}$ & $\begin{array}{c}-0.0125 \\
(0.0737)\end{array}$ & $\begin{array}{c}-0.0002 \\
(0.0024)\end{array}$ & $\begin{array}{c}0.0001 \\
(0.0019)\end{array}$ & $\begin{array}{c}0.0000 \\
(0.0003)\end{array}$ & $\begin{array}{c}-0.0018 \\
(0.0034)\end{array}$ \\
\hline $\mathrm{X}_{t} \mathbf{1}_{\text {Post }}$ & $\begin{array}{c}0.0612 \\
(0.0705)\end{array}$ & $\begin{array}{c}0.0005 \\
(0.0018)\end{array}$ & $\begin{array}{c}0.0014 \\
(0.0023)\end{array}$ & $\begin{array}{c}0.0002 \\
(0.0004)\end{array}$ & $\begin{array}{c}-0.0015 \\
(0.0022)\end{array}$ \\
\hline $\mathrm{X}_{t} \mathbf{1}_{\text {News }} \mathbf{1}_{\text {Post }}$ & $\begin{array}{c}0.1396 \\
(0.1015)\end{array}$ & $\begin{array}{c}0.0010 \\
(0.0062)\end{array}$ & $\begin{array}{c}0.0116 \\
(0.0117)\end{array}$ & $\begin{array}{c}0.0001 \\
(0.0009)\end{array}$ & $\begin{array}{c}-0.0006 \\
(0.0065)\end{array}$ \\
\hline $\operatorname{Sur}_{t} \mathbf{1}_{\text {Post }}$ & $\begin{array}{c}0.0185 \\
(0.0225)\end{array}$ & $\begin{array}{c}0.0278 \\
(0.0313)\end{array}$ & $\begin{array}{c}0.0257 \\
(0.0313)\end{array}$ & $\begin{array}{c}0.0239 \\
(0.0354)\end{array}$ & $\begin{array}{c}0.0287 \\
(0.0313)\end{array}$ \\
\hline Adjusted $\mathrm{R}^{2}$ & 0.3359 & 0.1848 & 0.2090 & 0.1808 & 0.1901 \\
\hline
\end{tabular}


TABLE 2.7: Realised Variance at 8:30 Announcements Using the MI Sample.

Results of regression of dependent variable $(R V)$ realised variance on liquidity measures: $S_{0}$ is spread, $D_{0}$ is the depth at the best quote, $D_{1}$ is the depth at the second best quote, AOF is the absolute order flow, AOI is the absolute order imbalance. Descriptions of the calculation of the variables can be found in Section 2.3. There are 176 observations in MI announcements sample. The superscripts ***,**, and *indicate significance at the $1 \%, 5 \%$, and $10 \%$ level respectively. The significance is assessed using Newey-West standard errors.

\begin{tabular}{|c|c|c|c|c|c|}
\hline \multirow{2}{*}{ Variables } & \multicolumn{5}{|c|}{ Liquidity Measure (MI Announcements Sample) } \\
\hline & $\mathbf{S}_{0}$ & $\mathbf{D}_{0}$ & $\mathbf{D}_{1}$ & AOF & AOI \\
\hline Intercept & $\begin{array}{l}0.0642^{* * *} \\
(0.0188)\end{array}$ & $\begin{array}{l}0.0775^{* * *} \\
(0.0190)\end{array}$ & $\begin{array}{l}0.0786^{* * *} \\
(0.0183)\end{array}$ & $\begin{array}{l}0.0687^{* * *} \\
(0.0152)\end{array}$ & $\begin{array}{l}0.0681^{* * *} \\
(0.0141)\end{array}$ \\
\hline $\mathbf{1}_{\text {News }}$ & $\begin{array}{c}0.0162 \\
(0.0433)\end{array}$ & $\begin{array}{c}-0.0006 \\
(0.0294)\end{array}$ & $\begin{array}{c}0.0003 \\
(0.0396)\end{array}$ & $\begin{array}{c}-0.0093 \\
(0.0266)\end{array}$ & $\begin{array}{c}0.0077 \\
(0.0242)\end{array}$ \\
\hline $\mathbf{1}_{\text {Post }}$ & $\begin{array}{c}-0.0317 \\
(0.0282)\end{array}$ & $\begin{array}{c}-0.0201 \\
(0.0241)\end{array}$ & $\begin{array}{c}-0.0253 \\
(0.0259)\end{array}$ & $\begin{array}{c}-0.0181 \\
(0.0197)\end{array}$ & $\begin{array}{r}-0.0134 \\
(0.0194)\end{array}$ \\
\hline $\mathbf{1}_{\text {News }} \mathbf{1}_{\text {Post }}$ & $\begin{array}{c}0.1007 \\
(0.1713)\end{array}$ & $\begin{array}{c}0.1791 \\
(0.1717)\end{array}$ & $\begin{array}{c}0.0494 \\
(0.1427)\end{array}$ & $\begin{array}{c}0.1917 \\
(0.1638)\end{array}$ & $\begin{array}{c}0.2195 \\
(0.1731)\end{array}$ \\
\hline $\mathrm{RV}_{t-15}$ & $\begin{array}{l}0.3821^{* * *} \\
(0.0420)\end{array}$ & $\begin{array}{l}0.3826^{* * *} \\
(0.0409)\end{array}$ & $\begin{array}{l}0.3810^{* * *} \\
(0.0421)\end{array}$ & $\begin{array}{l}0.3807^{* * *} \\
(0.0438)\end{array}$ & $\begin{array}{l}0.3847^{* * *} \\
(0.0408)\end{array}$ \\
\hline $\mathrm{RV}_{t-15} \mathbf{1}_{\text {News }}$ & $\begin{array}{c}-0.0650 \\
(0.2098)\end{array}$ & $\begin{array}{c}-0.0423 \\
(0.2234)\end{array}$ & $\begin{array}{c}-0.0317 \\
(0.2106)\end{array}$ & $\begin{array}{c}-0.2452 \\
(0.2259)\end{array}$ & $\begin{array}{r}-0.0554 \\
(0.2098)\end{array}$ \\
\hline $\mathrm{RV}_{t-15} \mathbf{1}_{\text {Post }}$ & $\begin{array}{c}0.1443 \\
(0.1021)\end{array}$ & $\begin{array}{c}0.1695 \\
(0.1149)\end{array}$ & $\begin{array}{c}0.1452 \\
(0.1045)\end{array}$ & $\begin{array}{c}0.0953 \\
(0.1739)\end{array}$ & $\begin{array}{c}0.1745 \\
(0.1160)\end{array}$ \\
\hline $\mathrm{RV}_{t-15} \mathbf{1}_{\text {News }} \mathbf{1}_{\text {Post }}$ & $\begin{array}{c}1.7000 \\
(1.0856)\end{array}$ & $\begin{array}{c}1.7010 \\
(1.8767)\end{array}$ & $\begin{array}{c}3.3298^{* *} \\
(1.4723)\end{array}$ & $\begin{array}{l}2.8365^{* *} \\
(1.5110)\end{array}$ & $\begin{array}{c}2.5675^{*} \\
(1.5887)\end{array}$ \\
\hline $\mathrm{X}_{t}$ & $\begin{array}{c}0.0095 \\
(0.0444)\end{array}$ & $\begin{array}{c}-0.0018 \\
(0.0015)\end{array}$ & $\begin{array}{c}-0.0017 \\
(0.0012)\end{array}$ & $\begin{array}{c}0.0000 \\
(0.0002)\end{array}$ & $\begin{array}{c}-0.0006 \\
(0.0017)\end{array}$ \\
\hline $\mathrm{X}_{t} \mathbf{1}_{\text {News }}$ & $\begin{array}{c}-0.0429 \\
(0.1193)\end{array}$ & $\begin{array}{c}0.0008 \\
(0.0022)\end{array}$ & $\begin{array}{c}0.0004 \\
(0.0041)\end{array}$ & $\begin{array}{c}0.0010 \\
(0.0006)\end{array}$ & $\begin{array}{c}-0.0010 \\
(0.0032)\end{array}$ \\
\hline $\mathrm{X}_{t} \mathbf{1}_{\text {Post }}$ & $\begin{array}{c}0.0612 \\
(0.0726)\end{array}$ & $\begin{array}{c}0.0005 \\
(0.0018)\end{array}$ & $\begin{array}{c}0.0014 \\
(0.0023)\end{array}$ & $\begin{array}{c}0.0002 \\
(0.0004)\end{array}$ & $\begin{array}{c}-0.0015 \\
(0.0023)\end{array}$ \\
\hline $\mathrm{X}_{t} \mathbf{1}_{\text {News }} \mathbf{1}_{\text {Post }}$ & $\begin{array}{c}0.1227 \\
(0.1412)\end{array}$ & $\begin{array}{c}0.0201 \\
(0.0333)\end{array}$ & $\begin{array}{c}0.230 \\
(0.0154)\end{array}$ & $\begin{array}{c}0.0004 \\
(0.0041)\end{array}$ & $\begin{array}{c}-0.0028 \\
(0.0287)\end{array}$ \\
\hline $\operatorname{Sur}_{t} \mathbf{1}_{\text {Post }}$ & $\begin{array}{c}-0.0414 \\
(0.1113)\end{array}$ & $\begin{array}{c}-0.1309 \\
(0.1172)\end{array}$ & $\begin{array}{c}-0.2385 \\
(0.1523)\end{array}$ & $\begin{array}{c}-0.1417 \\
(0.1312)\end{array}$ & $\begin{array}{r}-0.1414 \\
(0.1378)\end{array}$ \\
\hline Adjusted $\mathrm{R}^{2}$ & 0.4906 & 0.4139 & 0.4903 & 0.3984 & 0.3986 \\
\hline
\end{tabular}




\subsubsection{Realised Variance Regression Without Lag}

To clarify the impact of explanatory variables on realised variance, I evaluate the regression model for all three samples by excluding the realised variance lag. Table 2.8 shows that the results do not change after excluding realised variance lag and spread at the best quote remains the only significant variable among all liquidity variables as a proxy of private information shock. Surprise as a public information shock does not reveal any significant results even after excluding the realised variance lag. Tables 2.9 and 2.10 present the results for the relatively important and the most important news. There are not any significant results for these two samples since they are highly analysed by traders in the market.

\subsubsection{Realised Variance in the US and Canadian Market Separately}

In this section I examine the impact of 8:30 announcements on realised variance in the US and Canadian market separately. Table 2.1 determines the news days according to the US macroeconomic announcements and similarly Table 2.2 determines news days based on the Canadian macroeconomic announcements. I apply the same regression model (2.9) for the Full sample in each country. Table 2.11 reports the results for realised variance when I use only the US macroeconomic announcements. Spread as a private information shock is the only liquidity variable that is significant at the $5 \%$ level. Surprise as a public information shock does not reveal any significant results. A big portion of macroeconomic announcements are coming from the US and the results are not very different from Table 2.5. Table 2.12 reports the results when I use the Canadian macroeconomic announcements and results are neither significant for private information shocks nor for the public information shocks. It might imply that the US 
TABLE 2.8: Realised Variance at 8:30 Announcements Using the Full Sample, without Lag.

Results of regression of dependent variable $(R V)$ realised variance on liquidity measures: $S_{0}$ is spread, $D_{0}$ is the depth at the best quote, $D_{1}$ is the depth at the second best quote, AOF is the absolute order flow, AOI is the absolute order imbalance. Descriptions of the calculation of the variables can be found in Section 2.3. There are 330 observations in full announcements sample. The superscripts ***,**, and *indicate significance at the 1\%,5\%, and $10 \%$ level respectively. The significance is assessed using Newey-West standard errors.

\begin{tabular}{|c|c|c|c|c|c|}
\hline \multirow{2}{*}{ Variables } & \multicolumn{5}{|c|}{ Liquidity Measure Without Lag (Full Announcements Sample) } \\
\hline & $\mathbf{S}_{0}$ & $\mathbf{D}_{0}$ & $\mathbf{D}_{1}$ & AOF & AOI \\
\hline Intercept & $\begin{array}{l}0.0972^{* * *} \\
(0.0187)\end{array}$ & $\begin{array}{l}0.0210^{* * *} \\
(0.0210)\end{array}$ & $\begin{array}{l}0.0185^{* * *} \\
(0.0185)\end{array}$ & $\begin{array}{l}0.0155^{* * *} \\
(0.0155)\end{array}$ & $\begin{array}{l}0.0147^{* * *} \\
(0.0147)\end{array}$ \\
\hline $\mathbf{1}_{\text {News }}$ & $\begin{array}{c}0.0257 \\
(0.0264)\end{array}$ & $\begin{array}{c}0.0108 \\
(0.0248)\end{array}$ & $\begin{array}{c}0.0023 \\
(0.0233)\end{array}$ & $\begin{array}{c}0.0139 \\
(0.0190)\end{array}$ & $\begin{array}{c}0.0224 \\
(0.0181)\end{array}$ \\
\hline $\mathbf{1}_{\text {Post }}$ & $\begin{array}{c}-0.0133 \\
(0.0298)\end{array}$ & $\begin{array}{c}-0.0063 \\
(0.0274)\end{array}$ & $\begin{array}{c}0.0037 \\
(0.0271)\end{array}$ & $\begin{array}{c}-0.0150 \\
(0.0210)\end{array}$ & $\begin{array}{c}0.0065 \\
(0.0205)\end{array}$ \\
\hline $\mathbf{1}_{\text {News }} \mathbf{1}_{\text {Post }}$ & $\begin{array}{c}0.0577 \\
(0.0524)\end{array}$ & $\begin{array}{l}0.1674^{* *} \\
(0.0728)\end{array}$ & $\begin{array}{c}0.1156 \\
(0.0745)\end{array}$ & $\begin{array}{l}0.1692^{* *} \\
(0.0572)\end{array}$ & $\begin{array}{c}0.1477^{* *} \\
(0.0628)\end{array}$ \\
\hline$X_{t}$ & $\begin{array}{c}0.0092 \\
(0.0469)\end{array}$ & $\begin{array}{c}-0.0017 \\
(0.0023)\end{array}$ & $\begin{array}{c}-0.0018 \\
(0.0013)\end{array}$ & $\begin{array}{c}0.0003 \\
(0.0004)\end{array}$ & $\begin{array}{c}0.0008 \\
(0.0023)\end{array}$ \\
\hline $\mathrm{X}_{t} \mathbf{1}_{\text {News }}$ & $\begin{array}{c}-0.0460 \\
(0.0732)\end{array}$ & $\begin{array}{c}0.0003 \\
(0.0028)\end{array}$ & $\begin{array}{c}0.0015 \\
(0.0021)\end{array}$ & $\begin{array}{c}-0.0002 \\
(0.0004)\end{array}$ & $\begin{array}{c}-0.0038 \\
(0.0035)\end{array}$ \\
\hline $\mathrm{X}_{t} \mathbf{1}_{\text {Post }}$ & $\begin{array}{c}0.0649 \\
(0.0747)\end{array}$ & $\begin{array}{c}0.0017 \\
(0.0031)\end{array}$ & $\begin{array}{c}0.0001 \\
(0.0025)\end{array}$ & $\begin{array}{c}0.0006 \\
(0.0004)\end{array}$ & $\begin{array}{c}-0.0008 \\
(0.0040)\end{array}$ \\
\hline $\mathrm{X}_{t} \mathbf{1}_{\text {News }} \mathbf{1}_{\text {Post }}$ & $\begin{array}{l}0.2337^{* *} \\
(0.1111)\end{array}$ & $\begin{array}{c}-0.0038 \\
(0.0055)\end{array}$ & $\begin{array}{c}0.0041 \\
(0.0066)\end{array}$ & $\begin{array}{c}-0.0007 \\
(0.0005)\end{array}$ & $\begin{array}{c}-0.0005 \\
(0.0066)\end{array}$ \\
\hline $\operatorname{Sur}_{t} \mathbf{1}_{\text {Post }}$ & $\begin{array}{c}-0.0003 \\
(0.0217)\end{array}$ & $\begin{array}{c}0.0159 \\
(0.0260)\end{array}$ & $\begin{array}{c}0.0176 \\
(0.0264)\end{array}$ & $\begin{array}{c}0.0198 \\
(0.0282)\end{array}$ & $\begin{array}{c}0.0159 \\
(0.0262)\end{array}$ \\
\hline Adjusted $\mathrm{R}^{2}$ & 0.2724 & 0.1338 & 0.1350 & 0.1321 & 0.1346 \\
\hline
\end{tabular}


TABLE 2.9: Realised Variance at 8:30 Announcements Using the RI Sample, without Lag.

Results of regression of dependent variable $(R V)$ realised variance on liquidity measures: $S_{0}$ is spread, $D_{0}$ is the depth at the best quote, $D_{1}$ is the depth at the second best quote, AOF is the absolute order flow, AOI is the absolute order imbalance. Descriptions of the calculation of the variables can be found in Section 2.3. There are 240 observations in RI announcements sample. The superscripts ***,**, and *indicate significance at the 1\%,5\%, and $10 \%$ level respectively. The significance is assessed using Newey-West standard errors.

\begin{tabular}{|c|c|c|c|c|c|}
\hline \multirow{2}{*}{ Variables } & \multicolumn{5}{|c|}{ Liquidity Measure Without Lag (RI Announcements Sample) } \\
\hline & $\mathbf{S}_{0}$ & $\mathbf{D}_{0}$ & $\mathbf{D}_{1}$ & AOF & AOI \\
\hline Intercept & $\begin{array}{l}0.0972^{* * *} \\
(0.0188)\end{array}$ & $\begin{array}{l}0.1102^{* * *} \\
(0.0211)\end{array}$ & $\begin{array}{l}0.1122^{* * *} \\
(0.0186)\end{array}$ & $\begin{array}{l}0.0949^{* * *} \\
(0.0156)\end{array}$ & $\begin{array}{l}0.0974^{* * *} \\
(0.0148)\end{array}$ \\
\hline $\mathbf{1}_{\text {News }}$ & $\begin{array}{c}0.0171 \\
(0.0290)\end{array}$ & $\begin{array}{c}0.0149 \\
(0.0275)\end{array}$ & $\begin{array}{c}0.0144 \\
(0.0251)\end{array}$ & $\begin{array}{c}0.0160 \\
(0.0215)\end{array}$ & $\begin{array}{c}0.0219 \\
(0.0197)\end{array}$ \\
\hline $\mathbf{1}_{\text {Post }}$ & $\begin{array}{c}-0.0133 \\
(0.0300)\end{array}$ & $\begin{array}{r}-0.0063 \\
(0.0275)\end{array}$ & $\begin{array}{c}0.0037 \\
(0.0272)\end{array}$ & $\begin{array}{c}-0.0150 \\
(0.0211)\end{array}$ & $\begin{array}{c}0.0065 \\
(0.0206)\end{array}$ \\
\hline $\mathbf{1}_{\text {News }} \mathbf{1}_{\text {Post }}$ & $\begin{array}{c}0.0470 \\
(0.0570)\end{array}$ & $\begin{array}{c}0.1070^{*} \\
(0.0646)\end{array}$ & $\begin{array}{c}0.0251 \\
(0.0703)\end{array}$ & $\begin{array}{l}0.1307^{* *} \\
(0.0610)\end{array}$ & $\begin{array}{c}0.1142^{*} \\
(0.0619)\end{array}$ \\
\hline$X_{t}$ & $\begin{array}{c}0.0092 \\
(0.0472)\end{array}$ & $\begin{array}{r}-0.0017 \\
(0.0023)\end{array}$ & $\begin{array}{r}-0.0018 \\
(0.0013)\end{array}$ & $\begin{array}{c}0.0003 \\
(0.0004)\end{array}$ & $\begin{array}{c}0.0008 \\
(0.0024)\end{array}$ \\
\hline $\mathrm{X}_{t} \mathbf{1}_{\text {News }}$ & $\begin{array}{c}-0.0123 \\
(0.0776)\end{array}$ & $\begin{array}{c}-0.0004 \\
(0.0030)\end{array}$ & $\begin{array}{c}-0.0001 \\
(0.0021)\end{array}$ & $\begin{array}{c}-0.0002 \\
(0.0004)\end{array}$ & $\begin{array}{c}-0.0034 \\
(0.0039)\end{array}$ \\
\hline $\mathrm{X}_{t} \mathbf{1}_{\text {Post }}$ & $\begin{array}{c}0.0649 \\
(0.0751)\end{array}$ & $\begin{array}{c}0.0017 \\
(0.0032)\end{array}$ & $\begin{array}{c}0.0001 \\
(0.0025)\end{array}$ & $\begin{array}{c}0.0006 \\
(0.0004)\end{array}$ & $\begin{array}{c}-0.0008 \\
(0.0040)\end{array}$ \\
\hline $\mathrm{X}_{t} \mathbf{1}_{\text {News }} \mathbf{1}_{\text {Post }}$ & $\begin{array}{c}0.1377 \\
(0.1060)\end{array}$ & $\begin{array}{c}0.0016 \\
(0.0064)\end{array}$ & $\begin{array}{c}0.0150 \\
(0.0103)\end{array}$ & $\begin{array}{r}-0.0004 \\
(0.0010)\end{array}$ & $\begin{array}{c}0.0005 \\
(0.0068)\end{array}$ \\
\hline $\operatorname{Sur}_{t} \mathbf{1}_{\text {Post }}$ & $\begin{array}{c}0.0220 \\
(0.0247)\end{array}$ & $\begin{array}{c}0.0311 \\
(0.0335)\end{array}$ & $\begin{array}{c}0.0274 \\
(0.0326)\end{array}$ & $\begin{array}{c}0.0285 \\
(0.0373)\end{array}$ & $\begin{array}{c}0.0326 \\
(0.0336)\end{array}$ \\
\hline Adjusted $\mathrm{R}^{2}$ & 0.3116 & 0.1603 & 0.1960 & 0.1612 & 0.1619 \\
\hline
\end{tabular}


TABLE 2.10: Realised Variance at 8:30 Announcements Using the MI Sample, without Lag.

Results of regression of dependent variable ( $R V$ ) realised variance on liquidity measures: $S_{0}$ is spread, $D_{0}$ is the depth at the best quote, $D_{1}$ is the depth at the second best quote, $A O F$ is the absolute order flow, AOI is the absolute order imbalance. Descriptions of the calculation of the variables can be found in Section 2.3. There are 176 observations in MI announcements sample. The superscripts ***,**, and *indicate significance at the $1 \%, 5 \%$, and $10 \%$ level respectively. The significance is assessed using Newey-West standard errors.

\begin{tabular}{|c|c|c|c|c|c|}
\hline \multirow{2}{*}{ Variables } & \multicolumn{5}{|c|}{ Liquidity Measure Without Lag (MI Announcements Sample) } \\
\hline & $\mathbf{S}_{0}$ & $\mathbf{D}_{0}$ & $\mathbf{D}_{1}$ & AOF & AOI \\
\hline Intercept & $\begin{array}{l}0.0972^{* * *} \\
(0.0191)\end{array}$ & $\begin{array}{l}0.1102^{* * *} \\
(0.0215)\end{array}$ & $\begin{array}{l}0.1122^{* * *} \\
(0.0190)\end{array}$ & $\begin{array}{l}0.0949^{* * *} \\
(0.0159)\end{array}$ & $\begin{array}{l}0.0974^{* * *} \\
(0.0151)\end{array}$ \\
\hline $\mathbf{1}_{\text {News }}$ & $\begin{array}{c}0.0211 \\
(0.0392)\end{array}$ & $\begin{array}{c}-0.0080 \\
(0.0289)\end{array}$ & $\begin{array}{c}-0.0139 \\
(0.0407)\end{array}$ & $\begin{array}{c}-0.0302 \\
(0.0246)\end{array}$ & $\begin{array}{c}0.0055 \\
(0.0218)\end{array}$ \\
\hline $\mathbf{1}_{\text {Post }}$ & $\begin{array}{c}-0.0133 \\
(0.0306)\end{array}$ & $\begin{array}{c}-0.0063 \\
(0.0281)\end{array}$ & $\begin{array}{c}0.0037 \\
(0.0278)\end{array}$ & $\begin{array}{c}-0.0150 \\
(0.0215)\end{array}$ & $\begin{array}{c}0.0065 \\
(0.0210)\end{array}$ \\
\hline $\mathbf{1}_{\text {News }} \mathbf{1}_{\text {Post }}$ & $\begin{array}{c}0.1863 \\
(0.1800)\end{array}$ & $\begin{array}{c}0.2459 \\
(0.1696)\end{array}$ & $\begin{array}{l}0.3390^{* *} \\
(0.1601)\end{array}$ & $\begin{array}{l}0.4707^{* * *} \\
(0.1980)\end{array}$ & $\begin{array}{l}0.3701^{* *} \\
(0.1926)\end{array}$ \\
\hline$X_{t}$ & $\begin{array}{c}0.0092 \\
(0.0481)\end{array}$ & $\begin{array}{c}-0.0017 \\
(0.0023)\end{array}$ & $\begin{array}{c}-0.0018 \\
(0.0013)\end{array}$ & $\begin{array}{c}0.0003 \\
(0.0004)\end{array}$ & $\begin{array}{c}0.0008 \\
(0.0024)\end{array}$ \\
\hline $\mathrm{X}_{t} \mathbf{1}_{\text {News }}$ & $\begin{array}{c}-0.0989 \\
(0.1286)\end{array}$ & $\begin{array}{c}0.0008 \\
(0.0027)\end{array}$ & $\begin{array}{c}0.0015 \\
(0.0046)\end{array}$ & $\begin{array}{c}0.0009 \\
(0.0007)\end{array}$ & $\begin{array}{c}-0.0031 \\
(0.0040)\end{array}$ \\
\hline $\mathrm{X}_{t} \mathbf{1}_{\text {Post }}$ & $\begin{array}{c}0.0649 \\
(0.0765)\end{array}$ & $\begin{array}{c}0.0017 \\
(0.0032)\end{array}$ & $\begin{array}{c}0.0001 \\
(0.0026)\end{array}$ & $\begin{array}{c}0.0006 \\
(0.0004)\end{array}$ & $\begin{array}{c}-0.0008 \\
(0.0041)\end{array}$ \\
\hline $\mathrm{X}_{t} \mathbf{1}_{\text {News }} \mathbf{1}_{\text {Post }}$ & $\begin{array}{c}0.1986 \\
(0.1506)\end{array}$ & $\begin{array}{c}0.0326 \\
(0.0283)\end{array}$ & $\begin{array}{c}0.0157 \\
(0.0183)\end{array}$ & $\begin{array}{c}-0.0021 \\
(0.0045)\end{array}$ & $\begin{array}{c}0.0156 \\
(0.0301)\end{array}$ \\
\hline $\operatorname{Sur}_{t} \mathbf{1}_{\text {Post }}$ & $\begin{array}{c}0.0166 \\
(0.1014)\end{array}$ & $\begin{array}{c}-0.0903 \\
(0.1121)\end{array}$ & $\begin{array}{c}-0.1306 \\
(0.1360)\end{array}$ & $\begin{array}{c}-0.0753 \\
(0.1178)\end{array}$ & $\begin{array}{c}-0.0735 \\
(0.1168)\end{array}$ \\
\hline Adjusted $\mathrm{R}^{2}$ & 0.4399 & 0.3705 & 0.3569 & 0.3187 & 0.3190 \\
\hline
\end{tabular}


TABLE 2.11: Realised Variance at 8:30 Announcements in US, Using the Full Sample.

Results of regression of dependent variable $(R V)$ realised variance on liquidity measures: $S_{0}$ is spread, $D_{0}$ is the depth at the best quote, $D_{1}$ is the depth at the second best quote, AOF is the absolute order flow, AOI is the absolute order imbalance. Descriptions of the calculation of the variables can be found in Section 2.3. There are 321 observations in full announcements sample. The superscripts ***,**, and *indicate significance at the 1\%,5\%, and $10 \%$ level respectively. The significance is assessed using Newey-West standard errors.

\begin{tabular}{|c|c|c|c|c|c|}
\hline \multirow{2}{*}{ Variables } & \multicolumn{5}{|c|}{ Liquidity Measure in US (Full Announcements Sample) } \\
\hline & $\mathbf{S}_{0}$ & $\mathbf{D}_{0}$ & $\mathbf{D}_{1}$ & AOF & AOI \\
\hline Intercept & $\begin{array}{l}0.0743^{* * *} \\
(0.0185)\end{array}$ & $\begin{array}{l}0.0879^{* * *} \\
(0.0190)\end{array}$ & $\begin{array}{l}0.0889^{* * *} \\
(0.0182)\end{array}$ & $\begin{array}{l}0.0748^{* * *} \\
(0.0144)\end{array}$ & $\begin{array}{l}0.0756^{* * *} \\
(0.0143)\end{array}$ \\
\hline $\mathbf{1}_{\text {News }}$ & $\begin{array}{c}0.0248 \\
(0.0305)\end{array}$ & $\begin{array}{c}0.0007 \\
(0.0260)\end{array}$ & $\begin{array}{c}-0.0009 \\
(0.0266)\end{array}$ & $\begin{array}{c}0.0110 \\
(0.0205)\end{array}$ & $\begin{array}{c}0.0194 \\
(0.0208)\end{array}$ \\
\hline $\mathbf{1}_{\text {Post }}$ & $\begin{array}{c}-0.0418 \\
(0.0274)\end{array}$ & $\begin{array}{c}-0.0317 \\
(0.0238)\end{array}$ & $\begin{array}{c}-0.0332 \\
(0.0255)\end{array}$ & $\begin{array}{c}-0.0258 \\
(0.0189)\end{array}$ & $\begin{array}{c}-0.0219 \\
(0.0193)\end{array}$ \\
\hline $\mathbf{1}_{\text {News }} \mathbf{1}_{\text {Post }}$ & $\begin{array}{c}0.0606 \\
(0.0570)\end{array}$ & $\begin{array}{l}0.1752^{* * *} \\
(0.0722)\end{array}$ & $\begin{array}{c}0.1293^{*} \\
(0.0729)\end{array}$ & $\begin{array}{l}0.1473^{* * *} \\
(0.0601)\end{array}$ & $\begin{array}{l}0.1430^{* *} \\
(0.0636)\end{array}$ \\
\hline $\mathrm{RV}_{t-15}$ & $\begin{array}{l}0.3689^{* * *} \\
(0.0413)\end{array}$ & $\begin{array}{l}0.3694^{* * *} \\
(0.0401)\end{array}$ & $\begin{array}{l}0.3673^{* * *} \\
(0.0414)\end{array}$ & $\begin{array}{l}0.3745^{* * *} \\
(0.0421)\end{array}$ & $\begin{array}{l}0.3727^{* * *} \\
(0.0401)\end{array}$ \\
\hline $\mathrm{RV}_{t-15} \mathbf{1}_{\text {News }}$ & $\begin{array}{c}-0.0172 \\
(0.1588)\end{array}$ & $\begin{array}{c}-0.0085 \\
(0.1618)\end{array}$ & $\begin{array}{c}-0.0111 \\
(0.1611)\end{array}$ & $\begin{array}{c}-0.0300 \\
(0.1695)\end{array}$ & $\begin{array}{c}-0.0251 \\
(0.1564)\end{array}$ \\
\hline $\mathrm{RV}_{t-15} \mathbf{1}_{\text {Post }}$ & $\begin{array}{c}0.1671^{*} \\
(0.0968)\end{array}$ & $\begin{array}{c}0.1883^{*} \\
(0.1062)\end{array}$ & $\begin{array}{c}0.1623^{*} \\
(0.0995)\end{array}$ & $\begin{array}{c}0.1176 \\
(0.1505)\end{array}$ & $\begin{array}{c}0.1935^{*} \\
(0.1067)\end{array}$ \\
\hline $\mathrm{RV}_{t-15} \mathbf{1}_{\text {News }} \mathbf{1}_{\text {Post }}$ & $\begin{array}{c}-0.0628 \\
(0.3231)\end{array}$ & $\begin{array}{c}0.0334 \\
(0.3520)\end{array}$ & $\begin{array}{c}-0.0335 \\
(0.3600)\end{array}$ & $\begin{array}{c}0.0526 \\
(0.3806)\end{array}$ & $\begin{array}{c}0.0240 \\
(0.3411)\end{array}$ \\
\hline $\mathrm{X}_{t}$ & $\begin{array}{c}-0.0049 \\
(0.0470)\end{array}$ & $\begin{array}{c}-0.0024 \\
(0.0015)\end{array}$ & $\begin{array}{c}-0.0022^{* *} \\
(0.0011)\end{array}$ & $\begin{array}{c}-0.0001 \\
(0.0002)\end{array}$ & $\begin{array}{c}-0.0010 \\
(0.0017)\end{array}$ \\
\hline $\mathrm{X}_{t} \mathbf{1}_{\text {News }}$ & $\begin{array}{r}-0.0387 \\
(0.0739)\end{array}$ & $\begin{array}{c}0.0020 \\
(0.0023)\end{array}$ & $\begin{array}{c}0.0020 \\
(0.0019)\end{array}$ & $\begin{array}{c}0.0001 \\
(0.0002)\end{array}$ & $\begin{array}{c}-0.0024 \\
(0.0029)\end{array}$ \\
\hline $\mathrm{X}_{t} \mathbf{1}_{\text {Post }}$ & $\begin{array}{c}0.0697 \\
(0.0723)\end{array}$ & $\begin{array}{c}0.0011 \\
(0.0018)\end{array}$ & $\begin{array}{c}0.0014 \\
(0.0022)\end{array}$ & $\begin{array}{c}0.0003 \\
(0.0004)\end{array}$ & $\begin{array}{c}-0.0013 \\
(0.0022)\end{array}$ \\
\hline $\mathrm{X}_{t} \mathbf{1}_{\text {News }} \mathbf{1}_{\text {Post }}$ & $\begin{array}{l}0.2362^{* *} \\
(0.1105)\end{array}$ & $\begin{array}{c}-0.0065 \\
(0.0058)\end{array}$ & $\begin{array}{c}0.0021 \\
(0.0066)\end{array}$ & $\begin{array}{c}-0.0004 \\
(0.0004)\end{array}$ & $\begin{array}{c}-0.0013 \\
(0.0065)\end{array}$ \\
\hline $\operatorname{Sur}_{t} \mathbf{1}_{\text {Post }}$ & $\begin{array}{c}-0.0056 \\
(0.0205)\end{array}$ & $\begin{array}{c}0.0096 \\
(0.0245)\end{array}$ & $\begin{array}{c}0.0124 \\
(0.0248)\end{array}$ & $\begin{array}{c}0.0159 \\
(0.0264)\end{array}$ & $\begin{array}{c}0.0102 \\
(0.0247)\end{array}$ \\
\hline Adjusted $\mathrm{R}^{2}$ & 0.2826 & 0.1462 & 0.1424 & 0.1407 & 0.1459 \\
\hline
\end{tabular}

macroeconomic announcements are more important and include more private information shocks compared to the Canadian announcements. 
TABLE 2.12: Realised Variance at 8:30 Announcements in CA, Using the Full Sample.

Results of regression of dependent variable $(R V)$ realised variance on liquidity measures: $S_{0}$ is spread, $D_{0}$ is the depth at the best quote, $D_{1}$ is the depth at the second best quote, AOF is the absolute order flow, AOI is the absolute order imbalance. Descriptions of the calculation of the variables can be found in Section 2.3. There are 118 observations in full announcements sample. The superscripts ***,**, and *indicate significance at the $1 \%, 5 \%$, and $10 \%$ level respectively. The significance is assessed using Newey-West standard errors.

\begin{tabular}{|c|c|c|c|c|c|}
\hline \multirow{2}{*}{ Variables } & \multicolumn{5}{|c|}{ Liquidity Measure in CA (Full Announcements Sample) } \\
\hline & $\mathbf{S}_{0}$ & $\mathbf{D}_{0}$ & $\mathbf{D}_{1}$ & AOF & AOI \\
\hline Intercept & $\begin{array}{l}0.0624^{* * *} \\
(0.0183)\end{array}$ & $\begin{array}{l}0.0919^{* * *} \\
(0.0194)\end{array}$ & $\begin{array}{l}0.0884^{* * *} \\
(0.0186)\end{array}$ & $\begin{array}{l}0.0761^{* * *} \\
(0.0147)\end{array}$ & $\begin{array}{l}0.0786^{* * *} \\
(0.0145)\end{array}$ \\
\hline $\mathbf{1}_{\text {News }}$ & $\begin{array}{c}0.0459 \\
(0.0313)\end{array}$ & $\begin{array}{c}0.0075 \\
(0.0251)\end{array}$ & $\begin{array}{c}0.0059 \\
(0.0276)\end{array}$ & $\begin{array}{c}0.0157 \\
(0.0207)\end{array}$ & $\begin{array}{c}0.0208 \\
(0.0214)\end{array}$ \\
\hline $\mathbf{1}_{\text {Post }}$ & $\begin{array}{c}-0.0586 \\
(0.0397)\end{array}$ & $\begin{array}{c}-0.0171 \\
(0.0325)\end{array}$ & $\begin{array}{c}-0.0161 \\
(0.0482)\end{array}$ & $\begin{array}{c}-0.0227 \\
(0.0224)\end{array}$ & $\begin{array}{c}-0.0103 \\
(0.0269)\end{array}$ \\
\hline $\mathbf{1}_{\text {News }} \mathbf{1}_{\text {Post }}$ & $\begin{array}{c}0.0656 \\
(0.0654)\end{array}$ & $\begin{array}{c}0.1465^{*} \\
(0.0776)\end{array}$ & $\begin{array}{c}0.0994 \\
(0.0873)\end{array}$ & $\begin{array}{c}0.1310^{* *} \\
(0.0635)\end{array}$ & $\begin{array}{c}0.1229^{*} \\
(0.0685)\end{array}$ \\
\hline $\mathrm{RV}_{t-15}$ & $\begin{array}{l}0.3645^{* * *} \\
(0.0432)\end{array}$ & $\begin{array}{l}0.3650^{* * *} \\
(0.0406)\end{array}$ & $\begin{array}{l}0.3610^{* * *} \\
(0.0427)\end{array}$ & $\begin{array}{l}0.3701^{* * *} \\
(0.0416)\end{array}$ & $\begin{array}{l}0.3720^{* * *} \\
(0.0396)\end{array}$ \\
\hline $\mathrm{RV}_{t-15} \mathbf{1}_{\text {News }}$ & $\begin{array}{c}-0.1106 \\
(0.1699)\end{array}$ & $\begin{array}{c}-0.1121 \\
(0.1652)\end{array}$ & $\begin{array}{c}-0.1054 \\
(0.1673)\end{array}$ & $\begin{array}{c}-0.1347 \\
(0.1730)\end{array}$ & $\begin{array}{c}-0.1263 \\
(0.1647)\end{array}$ \\
\hline $\mathrm{RV}_{t-15} \mathbf{1}_{\text {Post }}$ & $\begin{array}{c}0.1972^{*} \\
(0.1164)\end{array}$ & $\begin{array}{c}0.2159^{*} \\
(0.1257)\end{array}$ & $\begin{array}{c}0.1772 \\
(0.1348)\end{array}$ & $\begin{array}{c}-0.1023 \\
(0.2803)\end{array}$ & $\begin{array}{r}0.2155^{*} \\
(0.1250)\end{array}$ \\
\hline $\mathrm{RV}_{t-15} \mathbf{1}_{\text {News }} \mathbf{1}_{\text {Post }}$ & $\begin{array}{c}0.1134 \\
(0.3533)\end{array}$ & $\begin{array}{c}0.2796 \\
(0.3706)\end{array}$ & $\begin{array}{c}0.1929 \\
(0.3969)\end{array}$ & $\begin{array}{c}0.5223 \\
(0.4709)\end{array}$ & $\begin{array}{c}0.2640 \\
(0.3611)\end{array}$ \\
\hline $\mathrm{X}_{t}$ & $\begin{array}{c}0.0459 \\
(0.0431)\end{array}$ & $\begin{array}{c}-0.0029^{*} \\
(0.0016)\end{array}$ & $\begin{array}{c}-0.0019^{*} \\
(0.0011)\end{array}$ & $\begin{array}{c}-0.0001 \\
(0.0002)\end{array}$ & $\begin{array}{c}-0.0018 \\
(0.0018)\end{array}$ \\
\hline $\mathrm{X}_{t} \mathbf{1}_{\text {News }}$ & $\begin{array}{c}-0.1009 \\
(0.0744)\end{array}$ & $\begin{array}{c}0.0019 \\
(0.0022)\end{array}$ & $\begin{array}{c}0.0018 \\
(0.0020)\end{array}$ & $\begin{array}{c}0.0001 \\
(0.0002)\end{array}$ & $\begin{array}{c}-0.0004 \\
(0.0032)\end{array}$ \\
\hline $\mathrm{X}_{t} \mathbf{1}_{\text {Post }}$ & $\begin{array}{c}0.1588 \\
(0.1581)\end{array}$ & $\begin{array}{c}0.0005 \\
(0.0022)\end{array}$ & $\begin{array}{c}0.0006 \\
(0.0040)\end{array}$ & $\begin{array}{c}0.0013 \\
(0.0010)\end{array}$ & $\begin{array}{c}-0.0015 \\
(0.0028)\end{array}$ \\
\hline $\mathrm{X}_{t} \mathbf{1}_{\text {News }} \mathbf{1}_{\text {Post }}$ & $\begin{array}{c}0.1523 \\
(0.1798)\end{array}$ & $\begin{array}{c}-0.0063 \\
(0.0061)\end{array}$ & $\begin{array}{c}0.0027 \\
(0.0076)\end{array}$ & $\begin{array}{c}-0.0015 \\
(0.0010)\end{array}$ & $\begin{array}{c}-0.0036 \\
(0.0071)\end{array}$ \\
\hline $\operatorname{Sur}_{t} \mathbf{1}_{\text {Post }}$ & $\begin{array}{c}-0.0067 \\
(0.0210)\end{array}$ & $\begin{array}{c}0.0085 \\
(0.0250)\end{array}$ & $\begin{array}{c}0.0115 \\
(0.0257)\end{array}$ & $\begin{array}{c}0.0155 \\
(0.0276)\end{array}$ & $\begin{array}{c}0.0085 \\
(0.0253)\end{array}$ \\
\hline Adjusted $R^{2}$ & 0.2855 & 0.1556 & 0.1481 & 0.1519 & 0.1551 \\
\hline
\end{tabular}




\subsubsection{Surprise Variable Analysis}

In this section, I provide more analysis on surprise variable as a proxy for public information shocks. The public information shocks do not show any significant results throughout the study. I drop this variable from my regression analysis to check if liquidity variables behave differently. Moreover, this is a test to examine what effect a purely private information shock might have on volatility. Table 2.13 shows that deleting the surprise from the regression model (2.9) does not change the behaviour of liquidity variables and their significance level. The reason might be due to the less important role of surprise in results and deleting this variable from the regression model does not cause any significant changes.

Next, I separate positive and negative surprises into two groups. I measure surprise as the difference between actual and survey values that is estimated by financial analyst (surprise=actual-survey). However, a positive difference has not the same meaning for all announcements. For example, more actual inflation than survey value is a bad news whereas more actual production than survey value is a good news. To have announcements with the same impact direction (e.g. good news), I set up actual minus survey for good news and survey minus actual for bad news. I count all announcements in Tables 2.1 and 2.2 as good news except Business Inventories, Wholesale Inventories MoM, Continuing Claims, Initial Jobless Claims, Unemployment Rate, Employment Cost Index, Unemployment Rate, PCE Core YoY, Import Price Index MoM CPI MoM, Industrial Production Price MoM, New Housing Price Index MoM, Raw materials Price Index MoM, and CPI YoY. In this regard, if the sign for all announcements (i.e. good/bad news) is positive we have positive shock otherwise is negative shock.

I revise the regression model (2.9) and separate the surprise variable into two components: positive and negative surprise. I define two dummy 
TABLE 2.13: Realised Variance at 8:30 Announcements Using the Full Sample with no Surprise.

Results of regression of dependent variable $(R V)$ realised variance on liquidity measures: $S_{0}$ is spread, $D_{0}$ is the depth at the best quote, $D_{1}$ is the depth at the second best quote, $A O F$ is the absolute order flow, AOI is the absolute order imbalance. Descriptions of the calculation of the variables can be found in Section 2.3. There are 330 observations in full announcements sample. The superscripts ***,**, and * indicate significance at the 1\%,5\%, and 10\% level respectively. The significance is assessed using Newey-West standard errors.

\begin{tabular}{|c|c|c|c|c|c|}
\hline \multirow{2}{*}{ Variables } & \multicolumn{5}{|c|}{ Liquidity Measure (Full Announcements Sample) } \\
\hline & $\mathbf{S}_{0}$ & $\mathbf{D}_{0}$ & $\mathbf{D}_{1}$ & AOF & AOI \\
\hline Intercept & $\begin{array}{l}0.0642^{* * *} \\
(0.0181)\end{array}$ & $\begin{array}{l}0.0775^{* * *} \\
(0.0183)\end{array}$ & $\begin{array}{l}0.0786^{* * *} \\
(0.0176)\end{array}$ & $\begin{array}{l}0.0687^{* * *} \\
(0.0146)\end{array}$ & $\begin{array}{l}0.0681^{* * *} \\
(0.0136)\end{array}$ \\
\hline $\mathbf{1}_{\text {News }}$ & $\begin{array}{c}0.0397 \\
(0.0300)\end{array}$ & $\begin{array}{c}0.0239 \\
(0.0241)\end{array}$ & $\begin{array}{c}0.0158 \\
(0.0266)\end{array}$ & $\begin{array}{c}0.0256 \\
(0.0205)\end{array}$ & $\begin{array}{c}0.0325 \\
(0.0204)\end{array}$ \\
\hline $\mathbf{1}_{\text {Post }}$ & $\begin{array}{c}-0.0317 \\
(0.0271)\end{array}$ & $\begin{array}{c}-0.0201 \\
(0.0232)\end{array}$ & $\begin{array}{c}-0.0253 \\
(0.0250)\end{array}$ & $\begin{array}{c}-0.0181 \\
(0.0190)\end{array}$ & $\begin{array}{c}-0.0134 \\
(0.0187)\end{array}$ \\
\hline $\mathbf{1}_{\text {News }} \mathbf{1}_{\text {Post }}$ & $\begin{array}{c}0.0351 \\
(0.0507)\end{array}$ & $\begin{array}{l}0.1604^{* * *} \\
(0.0554)\end{array}$ & $\begin{array}{l}0.1289^{* *} \\
(0.0575)\end{array}$ & $\begin{array}{l}0.1412^{* * *} \\
(0.0496)\end{array}$ & $\begin{array}{l}0.1376^{* * *} \\
(0.0476)\end{array}$ \\
\hline $\mathrm{RV}_{t-15}$ & $\begin{array}{l}0.3821^{* * *} \\
(0.0405)\end{array}$ & $\begin{array}{l}0.3826^{* * *} \\
(0.0394)\end{array}$ & $\begin{array}{l}0.3810^{* * *} \\
(0.0406)\end{array}$ & $\begin{array}{l}0.3807^{* * *} \\
(0.0421)\end{array}$ & $\begin{array}{l}0.3847^{* * *} \\
(0.0392)\end{array}$ \\
\hline $\mathrm{RV}_{t-15} \mathbf{1}_{\text {News }}$ & $\begin{array}{c}-0.1217 \\
(0.1652)\end{array}$ & $\begin{array}{c}-0.1255 \\
(0.1634)\end{array}$ & $\begin{array}{c}-0.1211 \\
(0.1648)\end{array}$ & $\begin{array}{c}-0.1510 \\
(0.1727)\end{array}$ & $\begin{array}{c}-0.1353 \\
(0.1627)\end{array}$ \\
\hline $\mathrm{RV}_{t-15} \mathbf{1}_{\text {Post }}$ & $\begin{array}{c}0.1443 \\
(0.0983)\end{array}$ & $\begin{array}{c}0.1695 \\
(0.1107)\end{array}$ & $\begin{array}{c}0.1452 \\
(0.1006)\end{array}$ & $\begin{array}{c}0.0953 \\
(0.1673)\end{array}$ & $\begin{array}{c}0.1745 \\
(0.1117)\end{array}$ \\
\hline $\mathrm{RV}_{t-15} \mathbf{1}_{\text {News }} \mathbf{1}_{\text {Post }}$ & $\begin{array}{c}0.1421 \\
(0.3379)\end{array}$ & $\begin{array}{c}0.2940 \\
(0.3535)\end{array}$ & $\begin{array}{c}0.1990 \\
(0.3758)\end{array}$ & $\begin{array}{c}0.3026 \\
(0.4029)\end{array}$ & $\begin{array}{c}0.2751 \\
(0.3456)\end{array}$ \\
\hline $\mathrm{X}_{t}$ & $\begin{array}{c}0.0095 \\
(0.0428)\end{array}$ & $\begin{array}{c}-0.0018 \\
(0.0014)\end{array}$ & $\begin{array}{c}-0.0017 \\
(0.0011)\end{array}$ & $\begin{array}{c}0.0000 \\
(0.0002)\end{array}$ & $\begin{array}{c}-0.0006 \\
(0.0016)\end{array}$ \\
\hline $\mathrm{X}_{t} \mathbf{1}_{\text {News }}$ & $\begin{array}{c}-0.0464 \\
(0.0702)\end{array}$ & $\begin{array}{c}0.0005 \\
(0.0021)\end{array}$ & $\begin{array}{c}0.0016 \\
(0.0020)\end{array}$ & $\begin{array}{c}0.0001 \\
(0.0002)\end{array}$ & $\begin{array}{c}-0.0020 \\
(0.0030)\end{array}$ \\
\hline $\mathrm{X}_{t} \mathbf{1}_{\text {Post }}$ & $\begin{array}{c}0.0612 \\
(0.0699)\end{array}$ & $\begin{array}{c}0.0005 \\
(0.0018)\end{array}$ & $\begin{array}{c}0.0014 \\
(0.0023)\end{array}$ & $\begin{array}{c}0.0002 \\
(0.0004)\end{array}$ & $\begin{array}{c}-0.0015 \\
(0.0022)\end{array}$ \\
\hline $\mathrm{X}_{t} \mathbf{1}_{\text {News }} \mathbf{1}_{\text {Post }}$ & $\begin{array}{l}0.2328^{* * *} \\
(0.1064)\end{array}$ & $\begin{array}{c}-0.0060 \\
(0.0058)\end{array}$ & $\begin{array}{c}0.0013 \\
(0.0066)\end{array}$ & $\begin{array}{c}-0.0003 \\
(0.0004)\end{array}$ & $\begin{array}{c}-0.0031 \\
(0.0067)\end{array}$ \\
\hline Adjusted $\mathrm{R}^{2}$ & 0.2952 & 0.1649 & 0.1575 & 0.1535 & 0.1646 \\
\hline
\end{tabular}


TABLE 2.14: Realised Variance at 8:30 Announcements Using the Full Sample with Positive and Negative Surprises.

Results of regression of dependent variable $(R V)$ realised variance on liquidity measures: $S_{0}$ is spread, $D_{0}$ is the depth at the best quote, $D_{1}$ is the depth at the second best quote, AOF is the absolute order flow, AOI is the absolute order imbalance. Descriptions of the calculation of the variables can be found in Section 2.3. There are 311 observations in full announcements sample. The superscripts ***,**, and *indicate significance at the 1\%,5\%, and $10 \%$ level respectively. The significance is assessed using Newey-West standard errors.

\begin{tabular}{|c|c|c|c|c|c|}
\hline \multirow{2}{*}{ Variables } & \multicolumn{5}{|c|}{ Liquidity Measure (Full Announcements Sample) } \\
\hline & $\mathbf{S}_{0}$ & $\mathbf{D}_{0}$ & $\mathbf{D}_{1}$ & AOF & AOI \\
\hline Intercept & $\begin{array}{l}0.0634^{* * *} \\
(0.0183)\end{array}$ & $\begin{array}{l}0.0804^{* * *} \\
(0.0194)\end{array}$ & $\begin{array}{l}0.0814^{* * *} \\
(0.0189)\end{array}$ & $\begin{array}{l}0.0687^{* * *} \\
(0.0147)\end{array}$ & $\begin{array}{l}0.0699^{* * *} \\
(0.0145)\end{array}$ \\
\hline $\mathbf{1}_{\text {News }}$ & $\begin{array}{c}0.0438 \\
(0.0306)\end{array}$ & $\begin{array}{c}0.0236 \\
(0.0251)\end{array}$ & $\begin{array}{c}0.0155 \\
(0.0277)\end{array}$ & $\begin{array}{c}0.0256 \\
(0.0205)\end{array}$ & $\begin{array}{c}0.0329 \\
(0.0212)\end{array}$ \\
\hline $\mathbf{1}_{\text {Post }}$ & $\begin{array}{c}-0.0308 \\
(0.0273)\end{array}$ & $\begin{array}{c}-0.0229 \\
(0.0241)\end{array}$ & $\begin{array}{c}-0.0281 \\
(0.0259)\end{array}$ & $\begin{array}{c}-0.0181 \\
(0.0190)\end{array}$ & $\begin{array}{c}-0.0152 \\
(0.0194)\end{array}$ \\
\hline $\mathbf{1}_{\text {News }} \mathbf{1}_{\text {Post }}$ & $\begin{array}{c}0.0391 \\
(0.0542)\end{array}$ & $\begin{array}{l}0.1563^{* *} \\
(0.0648)\end{array}$ & $\begin{array}{c}0.1276^{*} \\
(0.0706)\end{array}$ & $\begin{array}{l}0.1405^{* * *} \\
(0.0567)\end{array}$ & $\begin{array}{l}0.1334^{* *} \\
(0.0574)\end{array}$ \\
\hline $\mathrm{RV}_{t-15}$ & $\begin{array}{l}0.3779^{* * *} \\
(0.0421)\end{array}$ & $\begin{array}{l}0.3781^{* * *} \\
(0.0411)\end{array}$ & $\begin{array}{l}0.3759^{* * *} \\
(0.0425)\end{array}$ & $\begin{array}{l}0.3809^{* * *} \\
(0.0422)\end{array}$ & $\begin{array}{l}0.3813^{* * *} \\
(0.0407)\end{array}$ \\
\hline $\mathrm{RV}_{t-15} \mathbf{1}_{\text {News }}$ & $\begin{array}{c}-0.1330 \\
(0.1681)\end{array}$ & $\begin{array}{c}-0.1364 \\
(0.1655)\end{array}$ & $\begin{array}{c}-0.1307 \\
(0.1674)\end{array}$ & $\begin{array}{c}-0.1511 \\
(0.1740)\end{array}$ & $\begin{array}{c}-0.1476 \\
(0.1647)\end{array}$ \\
\hline $\mathrm{RV}_{t-15} \mathbf{1}_{\text {Post }}$ & $\begin{array}{c}0.1485 \\
(0.0993)\end{array}$ & $\begin{array}{c}0.1740 \\
(0.1116)\end{array}$ & $\begin{array}{c}0.1503 \\
(0.1017)\end{array}$ & $\begin{array}{c}0.0951 \\
(0.1678)\end{array}$ & $\begin{array}{c}0.1779 \\
(0.1125)\end{array}$ \\
\hline $\mathrm{RV}_{t-15} \mathbf{1}_{\text {News }} \mathbf{1}_{\text {Post }}$ & $\begin{array}{c}0.2022 \\
(0.3334)\end{array}$ & $\begin{array}{c}0.3680 \\
(0.3512)\end{array}$ & $\begin{array}{c}0.2801 \\
(0.3615)\end{array}$ & $\begin{array}{c}0.3860 \\
(0.3855)\end{array}$ & $\begin{array}{c}0.3492 \\
(0.3452)\end{array}$ \\
\hline$X_{t}$ & $\begin{array}{c}0.0191 \\
(0.0411)\end{array}$ & $\begin{array}{r}-0.0020 \\
(0.0015)\end{array}$ & $\begin{array}{c}-0.0018 \\
(0.0012)\end{array}$ & $\begin{array}{c}0.0000 \\
(0.0002)\end{array}$ & $\begin{array}{c}-0.0008 \\
(0.0016)\end{array}$ \\
\hline $\mathrm{X}_{t} \mathbf{1}_{\text {News }}$ & $\begin{array}{c}-0.0612 \\
(0.0702)\end{array}$ & $\begin{array}{c}0.0006 \\
(0.0021)\end{array}$ & $\begin{array}{c}0.0016 \\
(0.0020)\end{array}$ & $\begin{array}{c}0.0001 \\
(0.0002)\end{array}$ & $\begin{array}{c}-0.0020 \\
(0.0030)\end{array}$ \\
\hline $\mathrm{X}_{t} \mathbf{1}_{\text {Post }}$ & $\begin{array}{c}0.0516 \\
(0.0691)\end{array}$ & $\begin{array}{c}0.0007 \\
(0.0018)\end{array}$ & $\begin{array}{c}0.0015 \\
(0.0023)\end{array}$ & $\begin{array}{c}0.0002 \\
(0.0004)\end{array}$ & $\begin{array}{c}-0.0013 \\
(0.0022)\end{array}$ \\
\hline $\mathrm{X}_{t} \mathbf{1}_{\text {News }} \mathbf{1}_{\text {Post }}$ & $\begin{array}{c}0.2422 \\
(0.1065)\end{array}$ & $\begin{array}{r}-0.0057 \\
(0.0056)\end{array}$ & $\begin{array}{c}0.0010 \\
(0.0066)\end{array}$ & $\begin{array}{c}-0.0004 \\
(0.0005)\end{array}$ & $\begin{array}{c}-0.0026 \\
(0.0063)\end{array}$ \\
\hline $\operatorname{Sur}_{t} 1_{\text {Pos }} \mathbf{1}_{\text {Post }}$ & $\begin{array}{c}-0.0015 \\
(0.0174)\end{array}$ & $\begin{array}{c}0.0093 \\
(0.0232)\end{array}$ & $\begin{array}{c}0.0102 \\
(0.0241)\end{array}$ & $\begin{array}{c}0.0133 \\
(0.0249)\end{array}$ & $\begin{array}{c}0.0094 \\
(0.0235)\end{array}$ \\
\hline $\operatorname{Sur}_{t} 1_{N e g} \mathbf{1}_{\text {Post }}$ & $\begin{array}{c}0.0492 \\
(0.0463)\end{array}$ & $\begin{array}{c}0.0715 \\
(0.0484)\end{array}$ & $\begin{array}{c}0.0722 \\
(0.0523)\end{array}$ & $\begin{array}{c}0.0776 \\
(0.0534)\end{array}$ & $\begin{array}{c}0.0704 \\
(0.0485)\end{array}$ \\
\hline Adjusted $\mathrm{R}^{2}$ & 0.2924 & 0.1686 & 0.1617 & 0.1623 & 0.1680 \\
\hline
\end{tabular}


variables for each case. If the surprise is positive dummy is one otherwise is zero and a dummy in the same manner for negative surprises. Table 2.14 shows the results when I split the surprise into positive and negative. Results are robust with the main regression model and separating news will not change the significant level of other variables. Surprise as a public information shock did not reveal any significant role compared to private information shock and this separation will not change the relationship. Among all liquidity variables, spread as a proxy for private information shock is significant at the $5 \%$ level.

\subsubsection{Alternative Measures of Volatility}

In this section, I evaluate two alternative measures of realised variance: $A R$ and $R^{r e s i}$. I set up my regression model using (2.9), but I substitute $A R$ and $R^{r e s i}$ (as described in Section 2.2.3) in place of $R V$ as the dependent variable.

Many studies use the $A R$ measure to examine ex-post volatility (see Jones, Kaul, and Lipson (1994), Ahn, Bae, and K. Chan (2001), and Foucault, Moinas, and Theissen (2007)). In line with this literature, I use the $A R$ measure along with the three different announcement samples: Full, Relatively Important, and Most Important. However, the results are significant only for the Full and Relatively Important samples. Tables 2.15 and 2.16 report these results.

Table 2.15 shows that $A R$ is sensitive to spread and absolute order flow after the announcements on news days. As before, I capture this effect by interaction of $\mathbf{1}_{\text {News }}$ and $\mathbf{1}_{\text {post }}$ with explanatory variables. Spread (the difference between the best bid quote and the best ask quote) sensitivity has a positive coefficient and absolute order flow (the difference between the buy volume and the sell volume during the 15-minute interval) has a negative coefficient for the arrival of new information. I note that 
TABLE 2.15: Absolute Return at 8:30 Announcements Using the Full Sample.

Results of regression of dependent variable (AR) absolute return on liquidity measures: $S_{0}$ is spread, $D_{0}$ is the depth at the best quote, $D_{1}$ is the depth at the second best quote, AOF is the absolute order flow, AOI is the absolute order imbalance. Descriptions of the calculation of the variables can be found in Section 2.3. There are 330 observations in full announcements sample. The superscripts ***,**, and *indicate significance at the 1\%,5\%, and $10 \%$ level respectively. The significance is assessed using Newey-West standard errors. All numbers are scaled by 1000.

\begin{tabular}{|c|c|c|c|c|c|}
\hline \multirow{2}{*}{ Variables } & \multicolumn{5}{|c|}{ Liquidity Measure (Full Announcements Sample) } \\
\hline & $\mathbf{S}_{0}$ & $\mathbf{D}_{0}$ & $\mathbf{D}_{1}$ & AOF & AOI \\
\hline Intercept & $\begin{array}{c}0.1130^{*} \\
(0.0670)\end{array}$ & $\begin{array}{l}0.2200^{* * *} \\
(0.0620)\end{array}$ & $\begin{array}{l}0.2900^{* * *} \\
(0.0680)\end{array}$ & $\begin{array}{l}0.1890^{* * *} \\
(0.0380)\end{array}$ & $\begin{array}{l}0.1820^{* * *} \\
(0.0420)\end{array}$ \\
\hline $\mathbf{1}_{\text {News }}$ & $\begin{array}{c}0.1720^{*} \\
(0.0990)\end{array}$ & $\begin{array}{c}0.0370 \\
(0.0780)\end{array}$ & $\begin{array}{r}-0.0400 \\
(0.0870)\end{array}$ & $\begin{array}{c}0.0360 \\
(0.0530)\end{array}$ & $\begin{array}{c}0.0670 \\
(0.0570)\end{array}$ \\
\hline $\mathbf{1}_{\text {Post }}$ & $\begin{array}{c}0.1790^{*} \\
(0.0980)\end{array}$ & $\begin{array}{c}0.1280^{*} \\
(0.0780)\end{array}$ & $\begin{array}{c}0.0210 \\
(0.1090)\end{array}$ & $\begin{array}{c}0.1070^{*} \\
(0.0630)\end{array}$ & $\begin{array}{l}0.1510^{* * *} \\
(0.0610)\end{array}$ \\
\hline $\mathbf{1}_{\text {News }} \mathbf{1}_{\text {Post }}$ & $\begin{array}{r}-0.2300^{*} \\
(0.1320)\end{array}$ & $\begin{array}{r}-0.0900 \\
(0.1070)\end{array}$ & $\begin{array}{c}-0.0900 \\
(0.1390)\end{array}$ & $\begin{array}{r}-0.0300 \\
(0.0890)\end{array}$ & $\begin{array}{r}-0.0900 \\
(0.0890)\end{array}$ \\
\hline $\mathrm{AR}_{t-15}$ & $\begin{array}{c}225.2920 \\
(212.9000)\end{array}$ & $\begin{array}{c}186.3580 \\
(213.3000)\end{array}$ & $\begin{array}{c}241.9630 \\
(207.3000)\end{array}$ & $\begin{array}{c}403.0470 \\
(255.7000)\end{array}$ & $\begin{array}{c}166.5910 \\
(214.2000)\end{array}$ \\
\hline $\mathrm{AR}_{t-15} \mathbf{1}_{\text {News }}$ & $\begin{array}{c}-99.8100 \\
(243.9000)\end{array}$ & $\begin{array}{l}-78.6100 \\
(247.5000)\end{array}$ & $\begin{array}{r}-133.9100 \\
(242.9000)\end{array}$ & $\begin{array}{r}-375.5000 \\
(301.0000)\end{array}$ & $\begin{array}{l}-65.9700 \\
(248.2000)\end{array}$ \\
\hline $\mathrm{AR}_{t-15} \mathbf{1}_{\text {Post }}$ & $\begin{array}{r}-316.3100 \\
(259.2000)\end{array}$ & $\begin{array}{r}-220.6100 \\
(260.7000)\end{array}$ & $\begin{array}{r}-334.4500 \\
(255.9000)\end{array}$ & $\begin{array}{c}-618.1600^{* *} \\
(296.5000)\end{array}$ & $\begin{array}{r}-181.6400 \\
(262.6000)\end{array}$ \\
\hline $\mathrm{AR}_{t-15} \mathbf{1}_{\text {News }} \mathbf{1}_{\text {Post }}$ & $\begin{array}{c}334.6920 \\
(309.3000)\end{array}$ & $\begin{array}{c}218.4160 \\
(318.6000)\end{array}$ & $\begin{array}{c}340.7050 \\
(310.2000)\end{array}$ & $\begin{array}{c}669.8240^{* *} \\
(359.9000)\end{array}$ & $\begin{array}{c}218.9390 \\
(318.5000)\end{array}$ \\
\hline $\mathrm{X}_{t}$ & $\begin{array}{c}0.2410^{*} \\
(0.1290)\end{array}$ & $\begin{array}{r}-0.0066 \\
(0.0079)\end{array}$ & $\begin{array}{c}-0.0200^{* * *} \\
(0.0065)\end{array}$ & $\begin{array}{r}-0.0024 \\
(0.0015)\end{array}$ & $\begin{array}{c}0.0017 \\
(0.0090)\end{array}$ \\
\hline $\mathrm{X}_{t} \mathbf{1}_{\text {News }}$ & $\begin{array}{c}-0.4800^{* *} \\
(0.2380)\end{array}$ & $\begin{array}{c}0.0016 \\
(0.0092)\end{array}$ & $\begin{array}{c}0.0140^{*} \\
(0.0081)\end{array}$ & $\begin{array}{c}0.0028^{*} \\
(0.0016)\end{array}$ & $\begin{array}{c}-0.0098 \\
(0.0110)\end{array}$ \\
\hline $\mathrm{X}_{t} \mathbf{1}_{\text {Post }}$ & $\begin{array}{c}-0.1600 \\
(0.2300)\end{array}$ & $\begin{array}{c}-0.0003 \\
(0.0085)\end{array}$ & $\begin{array}{l}0.0180^{* *} \\
(0.0094)\end{array}$ & $\begin{array}{l}0.0039^{* *} \\
(0.0020)\end{array}$ & $\begin{array}{r}-0.0100 \\
(0.0100)\end{array}$ \\
\hline $\mathrm{X}_{t} \mathbf{1}_{\text {News }} \mathbf{1}_{\text {Post }}$ & $\begin{array}{c}0.5560^{*} \\
(0.3060)\end{array}$ & $\begin{array}{c}0.0045 \\
(0.0110)\end{array}$ & $\begin{array}{c}0.0005 \\
(0.0120)\end{array}$ & $\begin{array}{c}-0.0041^{* *} \\
(0.0020)\end{array}$ & $\begin{array}{c}0.0140 \\
(0.0130)\end{array}$ \\
\hline $\operatorname{Sur}_{t} \mathbf{1}_{\text {Post }}$ & $\begin{array}{c}0.0190 \\
(0.0270)\end{array}$ & $\begin{array}{c}0.0320 \\
(0.0310)\end{array}$ & $\begin{array}{c}0.0390 \\
(0.0320)\end{array}$ & $\begin{array}{c}0.0250 \\
(0.0340)\end{array}$ & $\begin{array}{c}0.0310 \\
(0.0310)\end{array}$ \\
\hline Adjusted $\mathrm{R}^{2}$ & 0.0070 & 0.0028 & 0.0069 & 0.0033 & 0.0033 \\
\hline
\end{tabular}

in both cases, the coefficient suggests volatility rises more when liquidity falls (i.e. spread rises, or order flow declines).

The results for spread and absolute order flow are significant at the 
TABLE 2.16: Absolute Return at 8:30 Announcements Using the RI Sample.

Results of regression of dependent variable (AR) absolute return on liquidity measures: $S_{0}$ is spread, $D_{0}$ is the depth at the best quote, $D_{1}$ is the depth at the second best quote, AOF is the absolute order flow, AOI is the absolute order imbalance. Descriptions of the calculation of the variables can be found in Section 2.3. There are 240 observations in RI announcements sample. The superscripts ***,**, and *indicate significance at the $1 \%, 5 \%$, and $10 \%$ level respectively. The significance is assessed using Newey-West standard errors. All numbers are scaled by 1000.

\begin{tabular}{|c|c|c|c|c|c|}
\hline \multirow{2}{*}{ Variables } & \multicolumn{5}{|c|}{ Liquidity Measure (RI Announcements Sample) } \\
\hline & $\mathbf{S}_{0}$ & $\mathbf{D}_{0}$ & $\mathbf{D}_{1}$ & AOF & AOI \\
\hline Intercept & $\begin{array}{c}0.1130^{*} \\
(0.0680)\end{array}$ & $\begin{array}{l}0.2200^{* * *} \\
(0.0620)\end{array}$ & $\begin{array}{l}0.2900^{* * *} \\
(0.0690)\end{array}$ & $\begin{array}{l}0.1890^{* * *} \\
(0.0390)\end{array}$ & $\begin{array}{l}0.1820^{* * *} \\
(0.0420)\end{array}$ \\
\hline $\mathbf{1}_{\text {News }}$ & $\begin{array}{c}0.1360 \\
(0.0900)\end{array}$ & $\begin{array}{c}0.0310 \\
(0.0780)\end{array}$ & $\begin{array}{c}-0.0700 \\
(0.0870)\end{array}$ & $\begin{array}{c}0.0210 \\
(0.0560)\end{array}$ & $\begin{array}{c}0.0560 \\
(0.0580)\end{array}$ \\
\hline $\mathbf{1}_{\text {Post }}$ & $\begin{array}{c}0.1790^{*} \\
(0.0990)\end{array}$ & $\begin{array}{c}0.1280^{*} \\
(0.0780)\end{array}$ & $\begin{array}{c}0.0210 \\
(0.1100)\end{array}$ & $\begin{array}{c}0.1070^{*} \\
(0.0630)\end{array}$ & $\begin{array}{l}0.1510^{* *} \\
(0.0620)\end{array}$ \\
\hline $\mathbf{1}_{\text {News }} \mathbf{1}_{\text {Post }}$ & $\begin{array}{r}-0.2100^{*} \\
(0.1310)\end{array}$ & $\begin{array}{c}-0.0700 \\
(0.1130)\end{array}$ & $\begin{array}{c}-0.1200 \\
(0.1440)\end{array}$ & $\begin{array}{c}-0.0200 \\
(0.1050)\end{array}$ & $\begin{array}{c}-0.0800 \\
(0.0970)\end{array}$ \\
\hline $\mathrm{AR}_{t-15}$ & $\begin{array}{c}225.2920 \\
(214.7000)\end{array}$ & $\begin{array}{c}186.3580 \\
(215.1000)\end{array}$ & $\begin{array}{c}241.9630 \\
(209.1000)\end{array}$ & $\begin{array}{c}403.0470 \\
(258.0000)\end{array}$ & $\begin{array}{c}166.5910 \\
(216.1000)\end{array}$ \\
\hline $\mathrm{AR}_{t-15} \mathbf{1}_{N e w s}$ & $\begin{array}{l}-36.9800 \\
(252.3000)\end{array}$ & $\begin{array}{c}-5.4600 \\
(252.5000)\end{array}$ & $\begin{array}{l}-58.0200 \\
(247.9000)\end{array}$ & $\begin{array}{r}-253.6300 \\
(298.8000)\end{array}$ & $\begin{array}{c}17.7010 \\
(253.2000)\end{array}$ \\
\hline $\mathrm{AR}_{t-15} \mathbf{1}_{\text {Post }}$ & $\begin{array}{c}-316.3100 \\
(261.4000)\end{array}$ & $\begin{array}{r}-220.6100 \\
(263.0000)\end{array}$ & $\begin{array}{r}-334.4500 \\
(258.1000)\end{array}$ & $\begin{array}{c}-618.1600^{* *} \\
(299.1000)\end{array}$ & $\begin{array}{r}-181.6400 \\
(264.9000)\end{array}$ \\
\hline $\mathrm{AR}_{t-15} \mathbf{1}_{\text {News }} \mathbf{1}_{\text {Post }}$ & $\begin{array}{c}193.2070 \\
(337.9000)\end{array}$ & $\begin{array}{c}67.0230 \\
(349.0000)\end{array}$ & $\begin{array}{c}139.5820 \\
(334.4000)\end{array}$ & $\begin{array}{c}471.0560 \\
(379.4000)\end{array}$ & $\begin{array}{c}51.6330 \\
(348.1000)\end{array}$ \\
\hline $\mathrm{X}_{t}$ & $\begin{array}{c}0.2410^{*} \\
(0.1310)\end{array}$ & $\begin{array}{c}-0.0066 \\
(0.0080)\end{array}$ & $\begin{array}{c}-0.0200^{* *} \\
(0.0066)\end{array}$ & $\begin{array}{r}-0.0024 \\
(0.0016)\end{array}$ & $\begin{array}{c}0.0017 \\
(0.0091)\end{array}$ \\
\hline $\mathrm{X}_{t} \mathbf{1}_{\text {News }}$ & $\begin{array}{c}-0.3600 \\
(0.2040)\end{array}$ & $\begin{array}{c}0.0008 \\
(0.0094)\end{array}$ & $\begin{array}{l}0.0170^{* *} \\
(0.0084)\end{array}$ & $\begin{array}{c}0.0029^{*} \\
(0.0018)\end{array}$ & $\begin{array}{c}-0.0100 \\
(0.0110)\end{array}$ \\
\hline $\mathrm{X}_{t} \mathbf{1}_{\text {Post }}$ & $\begin{array}{c}-0.1600 \\
(0.2320)\end{array}$ & $\begin{array}{c}-0.0003 \\
(0.0086)\end{array}$ & $\begin{array}{l}0.0180^{* *} \\
(0.0095)\end{array}$ & $\begin{array}{l}0.0039^{* *} \\
(0.0020)\end{array}$ & $\begin{array}{c}-0.0100 \\
(0.0100)\end{array}$ \\
\hline $\mathrm{X}_{t} \mathbf{1}_{\text {News }} \mathbf{1}_{\text {Post }}$ & $\begin{array}{c}0.4480^{*} \\
(0.2830)\end{array}$ & $\begin{array}{c}0.0039 \\
(0.0110)\end{array}$ & $\begin{array}{c}0.0140 \\
(0.0130)\end{array}$ & $\begin{array}{c}-0.0038 \\
(0.0024)\end{array}$ & $\begin{array}{c}0.0160 \\
(0.0130)\end{array}$ \\
\hline $\operatorname{Sur}_{t} \mathbf{1}_{\text {Post }}$ & $\begin{array}{c}0.0350 \\
(0.0370)\end{array}$ & $\begin{array}{c}0.0440 \\
(0.0420)\end{array}$ & $\begin{array}{c}0.0340 \\
(0.0390)\end{array}$ & $\begin{array}{c}0.0370 \\
(0.0450)\end{array}$ & $\begin{array}{c}0.0460 \\
(0.0430)\end{array}$ \\
\hline Adjusted $\mathrm{R}^{2}$ & 0.00810 & 0.0022 & 0.0143 & 0.0023 & 0.0028 \\
\hline
\end{tabular}


TABLE 2.17: Absolute Return at 8:30 Announcements Using the MI Sample.

Results of regression of dependent variable (AR) absolute return on liquidity measures: $S_{0}$ is spread, $D_{0}$ is the depth at the best quote, $D_{1}$ is the depth at the second best quote, AOF is the absolute order flow, AOI is the absolute order imbalance. Descriptions of the calculation of the variables can be found in Section 2.3. There are 176 observations in MI announcements sample. The superscripts ***,**, and *indicate significance at the $1 \%, 5 \%$, and $10 \%$ level respectively. The significance is assessed using Newey-West standard errors. All numbers are scaled by 1000.

\begin{tabular}{|c|c|c|c|c|c|}
\hline \multirow{2}{*}{ Variables } & \multicolumn{5}{|c|}{ Liquidity Measure (MI Announcements Sample) } \\
\hline & $\mathbf{S}_{0}$ & $\mathbf{D}_{0}$ & $\mathbf{D}_{1}$ & AOF & AOI \\
\hline Intercept & $\begin{array}{c}0.1130 \\
(0.0700)\end{array}$ & $\begin{array}{l}0.2200^{* * *} \\
(0.0640)\end{array}$ & $\begin{array}{l}0.2900^{* * *} \\
(0.0710)\end{array}$ & $\begin{array}{l}0.1890^{* * *} \\
(0.0400)\end{array}$ & $\begin{array}{l}0.1820^{* * *} \\
(0.0440)\end{array}$ \\
\hline $\mathbf{1}_{\text {News }}$ & $\begin{array}{c}-0.0200 \\
(0.2070)\end{array}$ & $\begin{array}{c}0.0220 \\
(0.1320)\end{array}$ & $\begin{array}{c}-0.2100 \\
(0.1090)\end{array}$ & $\begin{array}{c}-0.0200 \\
(0.0740)\end{array}$ & $\begin{array}{c}0.0570 \\
(0.1260)\end{array}$ \\
\hline $\mathbf{1}_{\text {Post }}$ & $\begin{array}{c}0.1790^{*} \\
(0.1030)\end{array}$ & $\begin{array}{c}0.1280 \\
(0.0810)\end{array}$ & $\begin{array}{c}0.0210 \\
(0.1140)\end{array}$ & $\begin{array}{c}0.1070^{*} \\
(0.0660)\end{array}$ & $\begin{array}{l}0.1510^{* *} \\
(0.0640)\end{array}$ \\
\hline $\mathbf{1}_{\text {News }} \mathbf{1}_{\text {Post }}$ & $\begin{array}{r}-0.2600^{*} \\
(0.3220)\end{array}$ & $\begin{array}{c}-0.2700 \\
(0.2380)\end{array}$ & $\begin{array}{c}0.1390 \\
(0.2630)\end{array}$ & $\begin{array}{c}-0.0200 \\
(0.2990)\end{array}$ & $\begin{array}{c}-0.2100 \\
(0.2270)\end{array}$ \\
\hline $\mathrm{AR}_{t-15}$ & $\begin{array}{c}225.2920 \\
(221.8000)\end{array}$ & $\begin{array}{c}186.3580 \\
(222.3000)\end{array}$ & $\begin{array}{c}241.9630 \\
(216.0000)\end{array}$ & $\begin{array}{c}403.0470 \\
(266.6000)\end{array}$ & $\begin{array}{c}166.5910 \\
(223.2000)\end{array}$ \\
\hline $\mathrm{AR}_{t-15} \mathbf{1}_{N e w s}$ & $\begin{array}{c}-51.5400 \\
(364.6000)\end{array}$ & $\begin{array}{r}-150.3000 \\
(324.4000)\end{array}$ & $\begin{array}{r}-353.3900 \\
(291.9000)\end{array}$ & $\begin{array}{r}-639.0400 \\
(366.8000)\end{array}$ & $\begin{array}{r}-129.3400 \\
(332.0000)\end{array}$ \\
\hline $\mathrm{AR}_{t-15} \mathbf{1}_{\text {Post }}$ & $\begin{array}{r}-316.3100 \\
(270.1000)\end{array}$ & $\begin{array}{r}-220.6100 \\
(271.7000)\end{array}$ & $\begin{array}{r}-334.4500 \\
(266.6000)\end{array}$ & $\begin{array}{c}-618.1600^{* *} \\
(309.1000)\end{array}$ & $\begin{array}{r}-181.6400 \\
(273.6000)\end{array}$ \\
\hline $\mathrm{AR}_{t-15} \mathbf{1}_{\text {News }} \mathbf{1}_{\text {Post }}$ & $\begin{array}{c}493.3440 \\
(680.6000)\end{array}$ & $\begin{array}{c}273.3010 \\
(632.5000)\end{array}$ & $\begin{array}{c}485.3050 \\
(621.5000)\end{array}$ & $\begin{array}{c}914.8790 \\
(790.1000)\end{array}$ & $\begin{array}{c}475.3600 \\
(703.5000)\end{array}$ \\
\hline $\mathrm{X}_{t}$ & $\begin{array}{c}0.2410^{*} \\
(0.1310)\end{array}$ & $\begin{array}{r}-0.0066^{*} \\
(0.0083)\end{array}$ & $\begin{array}{c}-0.0200^{* * *} \\
(0.0068)\end{array}$ & $\begin{array}{c}-0.0024 \\
(0.0016)\end{array}$ & $\begin{array}{c}0.0017 \\
(0.0094)\end{array}$ \\
\hline $\mathrm{X}_{t} \mathbf{1}_{\text {News }}$ & $\begin{array}{c}0.1940 \\
(0.5780)\end{array}$ & $\begin{array}{c}0.0058 \\
(0.0120)\end{array}$ & $\begin{array}{l}0.0450^{* * *} \\
(0.0160)\end{array}$ & $\begin{array}{l}0.0072^{* * *} \\
(0.0025)\end{array}$ & $\begin{array}{c}-0.0025 \\
(0.0240)\end{array}$ \\
\hline $\mathrm{X}_{t} \mathbf{1}_{\text {Post }}$ & $\begin{array}{c}-0.1600 \\
(0.2400)\end{array}$ & $\begin{array}{c}-0.0003 \\
(0.0088)\end{array}$ & $\begin{array}{c}0.0180^{*} \\
(0.0098)\end{array}$ & $\begin{array}{l}0.0039^{* *} \\
(0.0020)\end{array}$ & $\begin{array}{c}-0.0100 \\
(0.0100)\end{array}$ \\
\hline $\mathrm{X}_{t} \mathbf{1}_{\text {News }} \mathbf{1}_{\text {Post }}$ & $\begin{array}{c}-0.0900 \\
(0.6140)\end{array}$ & $\begin{array}{c}0.0420 \\
(0.0340)\end{array}$ & $\begin{array}{c}-0.0300 \\
(0.0240)\end{array}$ & $\begin{array}{c}-0.0061 \\
(0.0049)\end{array}$ & $\begin{array}{c}0.0460 \\
(0.0440)\end{array}$ \\
\hline $\operatorname{Sur}_{t} \mathbf{1}_{\text {Post }}$ & $\begin{array}{c}0.1470 \\
(0.2060)\end{array}$ & $\begin{array}{c}0.0410 \\
(0.2050)\end{array}$ & $\begin{array}{r}-0.0023 \\
(0.2180)\end{array}$ & $\begin{array}{c}0.0580 \\
(0.2000)\end{array}$ & $\begin{array}{c}0.0540 \\
(0.2080)\end{array}$ \\
\hline Adjusted $\mathrm{R}^{2}$ & 0.0089 & 0.0042 & 0.0045 & 0.0005 & -0.0006 \\
\hline
\end{tabular}


$10 \%$ and $5 \%$ level respectively. The results indicate that when I use the $A R$ measure, spread and absolute order flow become important microstructure variables (as compared to only spread when I use the Realised Variance measure). Surprise sensitivity as a proxy for public information shocks remains insignificant in all cases. The results for the Relatively Important sample (Table 2.16) show that the spread is the only significant liquidity measure. However, the (unreported) results for the Most Important sample are insignificant.

In high frequency data, there is a possibility that mid-quotes differ from their fair value due to market microstructure effects (Hasbrouck, 1993). My third measure, which is modified absolute return $\left(R^{\text {resi }}\right)$, regresses the absolute return of each interval on its lag value during each trading day (7:00 a.m-5:00 p.m) and retains the residuals (see Section 2.2.3). The absolute value of the retained residuals before and after 8:30 is the modified absolute return that is used as a proxy for measuring volatility (Foucault, Moinas, and Theissen, 2007). Tables 2.18 and 2.19 report my results for using the $R^{\text {resi }}$ measure for the Full and Relatively Important samples respectively.

Table 2.18 shows that the second alternative measure of volatility $\left(R^{r e s i}\right)$ after the announcements is sensitive to depth at the second best quote and absolute order flow, using the Full sample. I postulate that with the arrival of macroeconomic announcements and the existence of asymmetric information, traders avoid quoting at the best price to wait for the market to expose hidden information. Hence, a rise in the depth at the second best quote $\left(D_{1}\right)$ is also a symptom of illiquidity. In this regard, I note that realised variance has a significantly higher sensitivity to the depth behind the best quote $\left(D_{1}\right)$. The results indicate that depth at the second best quote $\left(D_{1}\right)$ and absolute order flow $(A O F)$ are significant at the $1 \%$ and $5 \%$ level (respectively) for the Full sample. Absolute order flow declines when the market is less liquid. Hence my negative coeffi- 
TABLE 2.18: Modified Absolute Return at 8:30 Announcements Using the Full Sample.

Results of regression of dependent variable $\left(R^{\text {resi }}\right)$ modified absolute return on liquidity measures: $S_{0}$ is spread, $D_{0}$ is the depth at the best quote, $D_{1}$ is the depth at the second best quote, $A O F$ is the absolute order flow, $A O I$ is the absolute order imbalance. Descriptions of the calculation of the variables can be found in Section 2.3. There are 330 observations in full announcements sample. The superscripts ***,**, and * indicate significance at the 1\%, 5\%, and $10 \%$ level respectively. The significance is assessed using Newey-West standard errors. All numbers are scaled by 1000.

\begin{tabular}{|c|c|c|c|c|c|}
\hline \multirow{2}{*}{ Variables } & \multicolumn{5}{|c|}{ Liquidity Measure (Full Announcements Sample) } \\
\hline & $\mathbf{S}_{0}$ & $\mathbf{D}_{0}$ & $\mathbf{D}_{1}$ & AOF & AOI \\
\hline Intercept & $\begin{array}{c}0.0760^{* *} \\
(0.0330)\end{array}$ & $\begin{array}{l}0.0570^{* *} \\
(0.0260)\end{array}$ & $\begin{array}{c}0.0380 \\
(0.0290)\end{array}$ & $\begin{array}{l}0.0780^{* * *} \\
(0.0280)\end{array}$ & $\begin{array}{l}0.0670^{* * *} \\
(0.0290)\end{array}$ \\
\hline $\mathbf{1}_{\text {News }}$ & $\begin{array}{c}0.1230^{* *} \\
(0.0640)\end{array}$ & $\begin{array}{c}0.0930^{*} \\
(0.0530)\end{array}$ & $\begin{array}{l}0.1450^{* * *} \\
(0.0440)\end{array}$ & $\begin{array}{l}0.0910^{* * *} \\
(0.0360)\end{array}$ & $\begin{array}{l}0.1160^{* * *} \\
(0.0380)\end{array}$ \\
\hline $\mathbf{1}_{\text {Post }}$ & $\begin{array}{c}0.0062 \\
(0.0530)\end{array}$ & $\begin{array}{c}0.0440 \\
(0.0370)\end{array}$ & $\begin{array}{l}0.0910^{* *} \\
(0.0460)\end{array}$ & $\begin{array}{c}-0.0200 \\
(0.0350)\end{array}$ & $\begin{array}{c}0.0130 \\
(0.0390)\end{array}$ \\
\hline $\mathbf{1}_{\text {News }} \mathbf{1}_{\text {Post }}$ & $\begin{array}{c}-0.0800 \\
(0.0830)\end{array}$ & $\begin{array}{c}-0.0200 \\
(0.0710)\end{array}$ & $\begin{array}{c}-0.2000^{* * *} \\
(0.0760)\end{array}$ & $\begin{array}{c}0.0290 \\
(0.0550)\end{array}$ & $\begin{array}{c}-0.0100 \\
(0.0590)\end{array}$ \\
\hline $\mathrm{R}_{t-15}^{r e s i}$ & $\begin{array}{c}373.1200^{* *} \\
(165.1000)\end{array}$ & $\begin{array}{c}260.8360 \\
(261.1000)\end{array}$ & $\begin{array}{c}354.7160^{* *} \\
(174.7000)\end{array}$ & $\begin{array}{c}345.0530^{* *} \\
(182.6000)\end{array}$ & $\begin{array}{c}336.5190 \\
(276.0000)\end{array}$ \\
\hline $\mathrm{R}_{t-15}^{r e s i} \mathbf{1}_{\text {News }}$ & $\begin{array}{r}-365.8800^{*} \\
(209.8000)\end{array}$ & $\begin{array}{c}-254.5600 \\
(290.0000)\end{array}$ & $\begin{array}{c}-340.9100 \\
(217.2000)\end{array}$ & $\begin{array}{c}-332.0600 \\
(241.4000)\end{array}$ & $\begin{array}{c}-336.3600 \\
(303.8000)\end{array}$ \\
\hline $\mathrm{R}_{t-15}^{r e s i} \mathbf{1}_{\text {Post }}$ & $\begin{array}{c}155.7180 \\
(225.0000)\end{array}$ & $\begin{array}{c}254.6440 \\
(302.5000)\end{array}$ & $\begin{array}{c}221.1350 \\
(217.1000)\end{array}$ & $\begin{array}{c}17.1030 \\
(250.0000)\end{array}$ & $\begin{array}{c}195.5450 \\
(313.4000)\end{array}$ \\
\hline $\mathbf{R}_{t-15}^{\text {resi }} \mathbf{1}_{\text {News }} \mathbf{1}_{\text {Post }}$ & $\begin{array}{c}2.4110 \\
(275.8000)\end{array}$ & $\begin{array}{c}-103.6400 \\
(345.7000)\end{array}$ & $\begin{array}{l}-61.9600 \\
(270.0000)\end{array}$ & $\begin{array}{c}139.9820 \\
(311.5000)\end{array}$ & $\begin{array}{l}-15.4400 \\
(353.2000)\end{array}$ \\
\hline $\mathrm{X}_{t}$ & $\begin{array}{c}-0.0400 \\
(0.0470)\end{array}$ & $\begin{array}{c}0.0038 \\
(0.0042)\end{array}$ & $\begin{array}{c}0.0039 \\
(0.0027)\end{array}$ & $\begin{array}{c}-0.0003 \\
(0.0007)\end{array}$ & $\begin{array}{c}0.0011 \\
(0.0045)\end{array}$ \\
\hline $\mathrm{X}_{t} \mathbf{1}_{\text {News }}$ & $\begin{array}{c}-0.0700 \\
(0.1740)\end{array}$ & $\begin{array}{c}-0.0006 \\
(0.0078)\end{array}$ & $\begin{array}{c}-0.0060 \\
(0.0040)\end{array}$ & $\begin{array}{c}0.0003 \\
(0.0007)\end{array}$ & $\begin{array}{c}-0.0070 \\
(0.0070)\end{array}$ \\
\hline $\mathrm{X}_{t} \mathbf{1}_{\text {Post }}$ & $\begin{array}{c}0.0420 \\
(0.0990)\end{array}$ & $\begin{array}{c}-0.0078 \\
(0.0055)\end{array}$ & $\begin{array}{c}-0.0100^{* * *} \\
(0.0042)\end{array}$ & $\begin{array}{l}0.0018^{* *} \\
(0.0008)\end{array}$ & $\begin{array}{c}-0.0001 \\
(0.0120)\end{array}$ \\
\hline $\mathrm{X}_{t} \mathbf{1}_{\text {News }} \mathbf{1}_{\text {Post }}$ & $\begin{array}{c}0.2140 \\
(0.1970)\end{array}$ & $\begin{array}{c}0.0051 \\
(0.0095)\end{array}$ & $\begin{array}{l}0.0260^{* * *} \\
(0.0069)\end{array}$ & $\begin{array}{c}-0.0019^{* *} \\
(0.0009)\end{array}$ & $\begin{array}{c}0.0028 \\
(0.0130)\end{array}$ \\
\hline $\operatorname{Sur}_{t} \mathbf{1}_{\text {Post }}$ & $\begin{array}{c}0.0140 \\
(0.0220)\end{array}$ & $\begin{array}{c}0.0250 \\
(0.0260)\end{array}$ & $\begin{array}{c}0.0300 \\
(0.0270)\end{array}$ & $\begin{array}{c}0.0280 \\
(0.0290)\end{array}$ & $\begin{array}{c}0.0240 \\
(0.0260)\end{array}$ \\
\hline Adjusted $\mathrm{R}^{2}$ & 0.0079 & 0.0021 & 0.0089 & 0.0020 & 0.0024 \\
\hline
\end{tabular}


TABLE 2.19: Modified Absolute Return at 8:30 Announcements Using the RI Sample.

Results of regression of dependent variable $\left(R^{\text {resi }}\right)$ modified absolute return on liquidity measures: $S_{0}$ is spread, $D_{0}$ is the depth at the best quote, $D_{1}$ is the depth at the second best quote, $A O F$ is the absolute order flow, AOI is the absolute order imbalance. Descriptions of the calculation of the variables can be found in Section 2.3. There are 240 observations in RI announcements sample. The superscripts ${ }^{* * *}, * *$, and ${ }^{*}$ indicate significance at the $1 \%, 5 \%$, and $10 \%$ level respectively. The significance is assessed using Newey-West standard errors. All numbers are scaled by 1000.

\begin{tabular}{|c|c|c|c|c|c|}
\hline \multirow{2}{*}{ Variables } & \multicolumn{5}{|c|}{ Liquidity Measure (RI Announcements Sample) } \\
\hline & $\mathbf{S}_{0}$ & $\mathbf{D}_{0}$ & $\mathbf{D}_{1}$ & AOF & AOI \\
\hline Intercept & $\begin{array}{l}0.0730^{* *} \\
(0.0330)\end{array}$ & $\begin{array}{l}0.0530^{* *} \\
(0.0260)\end{array}$ & $\begin{array}{c}0.0350 \\
(0.0290)\end{array}$ & $\begin{array}{l}0.0740^{* * *} \\
(0.0280)\end{array}$ & $\begin{array}{l}0.0640^{* *} \\
(0.0290)\end{array}$ \\
\hline $\mathbf{1}_{\text {News }}$ & $\begin{array}{c}0.1110^{*} \\
(0.0610)\end{array}$ & $\begin{array}{c}0.0420 \\
(0.0660)\end{array}$ & $\begin{array}{l}0.1020^{* *} \\
(0.0440)\end{array}$ & $\begin{array}{c}0.0710^{*} \\
(0.0410)\end{array}$ & $\begin{array}{l}0.1010^{* *} \\
(0.0420)\end{array}$ \\
\hline $\mathbf{1}_{\text {Post }}$ & $\begin{array}{c}0.0094 \\
(0.0540)\end{array}$ & $\begin{array}{c}0.0470 \\
(0.0370)\end{array}$ & $\begin{array}{l}0.0950^{* *} \\
(0.0460)\end{array}$ & $\begin{array}{c}-0.0200 \\
(0.0350)\end{array}$ & $\begin{array}{c}0.0160 \\
(0.0390)\end{array}$ \\
\hline $\mathbf{1}_{\text {News }} \mathbf{1}_{\text {Post }}$ & $\begin{array}{c}-0.1000 \\
(0.0840)\end{array}$ & $\begin{array}{c}0.0110 \\
(0.0840)\end{array}$ & $\begin{array}{c}-0.2300^{* * *} \\
(0.0800)\end{array}$ & $\begin{array}{c}0.0340 \\
(0.0680)\end{array}$ & $\begin{array}{c}-0.0200 \\
(0.0660)\end{array}$ \\
\hline $\mathrm{R}_{t-15}^{r e s i}$ & $\begin{array}{c}401.0010^{* *} \\
(166.5000)\end{array}$ & $\begin{array}{c}282.9250 \\
(265.8000)\end{array}$ & $\begin{array}{c}381.8710^{* *} \\
(176.9000)\end{array}$ & $\begin{array}{c}373.5410^{* *} \\
(185.4000)\end{array}$ & $\begin{array}{c}360.2420 \\
(280.6000)\end{array}$ \\
\hline $\mathrm{R}_{t-15}^{\text {resi }} \mathbf{1}_{\text {News }}$ & $\begin{array}{r}-313.8700 \\
(256.9000)\end{array}$ & $\begin{array}{c}-197.0500 \\
(325.2000)\end{array}$ & $\begin{array}{c}-306.4500 \\
(262.7000)\end{array}$ & $\begin{array}{c}-315.0000 \\
(274.0000)\end{array}$ & $\begin{array}{c}-289.5700 \\
(339.1000)\end{array}$ \\
\hline $\mathrm{R}_{t-15}^{\text {resi }} \mathbf{1}_{\text {Post }}$ & $\begin{array}{c}124.1140 \\
(229.7000)\end{array}$ & $\begin{array}{c}229.2650 \\
(309.3000)\end{array}$ & $\begin{array}{c}187.9950 \\
(222.3000)\end{array}$ & $\begin{array}{l}-23.4600 \\
(251.5000)\end{array}$ & $\begin{array}{c}169.2870 \\
(319.6000)\end{array}$ \\
\hline $\mathbf{R}_{t-15}^{r e s i} \mathbf{1}_{\text {News }} \mathbf{1}_{\text {Post }}$ & $\begin{array}{c}43.6650 \\
(316.4000)\end{array}$ & $\begin{array}{l}-51.1200 \\
(377.2000)\end{array}$ & $\begin{array}{l}-14.3200 \\
(309.5000)\end{array}$ & $\begin{array}{c}225.8660 \\
(342.6000)\end{array}$ & $\begin{array}{c}40.5760 \\
(386.2000)\end{array}$ \\
\hline $\mathrm{X}_{t}$ & $\begin{array}{c}-0.0400 \\
(0.0470)\end{array}$ & $\begin{array}{c}0.0040 \\
(0.0043)\end{array}$ & $\begin{array}{c}0.0039 \\
(0.0027)\end{array}$ & $\begin{array}{c}-0.0003 \\
(0.0007)\end{array}$ & $\begin{array}{c}0.0012 \\
(0.0046)\end{array}$ \\
\hline $\mathrm{X}_{t} \mathbf{1}_{\text {News }}$ & $\begin{array}{c}-0.0800 \\
(0.1710)\end{array}$ & $\begin{array}{c}0.0056 \\
(0.0100)\end{array}$ & $\begin{array}{c}-0.0017 \\
(0.0041)\end{array}$ & $\begin{array}{c}0.0005 \\
(0.0008)\end{array}$ & $\begin{array}{c}-0.0071 \\
(0.0088)\end{array}$ \\
\hline $\mathrm{X}_{t} \mathbf{1}_{\text {Post }}$ & $\begin{array}{c}0.0440 \\
(0.1000)\end{array}$ & $\begin{array}{c}-0.0077 \\
(0.0056)\end{array}$ & $\begin{array}{c}-0.0100^{* *} \\
(0.0042)\end{array}$ & $\begin{array}{l}0.0018^{* *} \\
(0.0009)\end{array}$ & $\begin{array}{c}0.0003 \\
(0.0120)\end{array}$ \\
\hline $\mathrm{X}_{t} \mathbf{1}_{\text {News }} \mathbf{1}_{\text {Post }}$ & $\begin{array}{c}0.2210 \\
(0.1950)\end{array}$ & $\begin{array}{c}-0.0025 \\
(0.0120)\end{array}$ & $\begin{array}{l}0.0330^{* * *} \\
(0.0082)\end{array}$ & $\begin{array}{r}-0.0025^{*} \\
(0.0014)\end{array}$ & $\begin{array}{c}0.0021 \\
(0.0150)\end{array}$ \\
\hline $\operatorname{Sur}_{t} \mathbf{1}_{\text {Post }}$ & $\begin{array}{c}0.0320 \\
(0.0330)\end{array}$ & $\begin{array}{c}0.0390 \\
(0.0390)\end{array}$ & $\begin{array}{c}0.0310 \\
(0.0360)\end{array}$ & $\begin{array}{c}0.0450 \\
(0.0390)\end{array}$ & $\begin{array}{c}0.0400 \\
(0.0390)\end{array}$ \\
\hline Adjusted $\mathrm{R}^{2}$ & 0.0126 & 0.0053 & 0.0182 & 0.0041 & 0.0044 \\
\hline
\end{tabular}

cient is consistent: volatility becomes more sensitive to liquidity declines post-announcement. Similarly, Table 2.19 shows that the $R^{\text {resi }}$ measure is sensitive to depth at the second best quote and absolute order flow after 
TABLE 2.20: Modified Absolute Return at 8:30 Announcements Using the MI Sample.

Results of regression of dependent variable $\left(R^{\text {resi }}\right)$ modified absolute return on liquidity measures: $S_{0}$ is spread, $D_{0}$ is the depth at the best quote, $D_{1}$ is the depth at the second best quote, $A O F$ is the absolute order flow, AOI is the absolute order imbalance. Descriptions of the calculation of the variables can be found in Section 2.3. There are 176 observations in MI announcements sample. The superscripts ***,**, and *indicate significance at the 1\%,5\%, and $10 \%$ level respectively. The significance is assessed using Newey-West standard errors. All numbers are scaled by 1000.

\begin{tabular}{|c|c|c|c|c|c|}
\hline \multirow{2}{*}{ Variables } & \multicolumn{5}{|c|}{ Liquidity Measure (MI Announcements Sample) } \\
\hline & $\mathbf{S}_{0}$ & $\mathbf{D}_{0}$ & $\mathbf{D}_{1}$ & AOF & AOI \\
\hline Intercept & $\begin{array}{l}0.0720^{* *} \\
(0.0350)\end{array}$ & $\begin{array}{c}0.0530^{* *} \\
(0.0280)\end{array}$ & $\begin{array}{c}0.0360 \\
(0.0300)\end{array}$ & $\begin{array}{l}0.0740^{* * *} \\
(0.0290)\end{array}$ & $\begin{array}{l}0.0630^{* *} \\
(0.0300)\end{array}$ \\
\hline $\mathbf{1}_{\text {News }}$ & $\begin{array}{c}0.0800 \\
(0.0750)\end{array}$ & $\begin{array}{c}0.0450 \\
(0.0700)\end{array}$ & $\begin{array}{c}0.0440 \\
(0.0680)\end{array}$ & $\begin{array}{c}0.0210 \\
(0.0480)\end{array}$ & $\begin{array}{c}0.0390 \\
(0.0660)\end{array}$ \\
\hline $\mathbf{1}_{\text {Post }}$ & $\begin{array}{c}0.0095 \\
(0.0570)\end{array}$ & $\begin{array}{c}0.0470 \\
(0.0390)\end{array}$ & $\begin{array}{l}0.0930^{* *} \\
(0.0480)\end{array}$ & $\begin{array}{r}-0.0200 \\
(0.0360)\end{array}$ & $\begin{array}{c}0.0160 \\
(0.0410)\end{array}$ \\
\hline $\mathbf{1}_{\text {News }} \mathbf{1}_{\text {Post }}$ & $\begin{array}{r}-0.0700 \\
(0.2150)\end{array}$ & $\begin{array}{r}-0.1600 \\
(0.1580)\end{array}$ & $\begin{array}{c}-0.0800 \\
(0.1860)\end{array}$ & $\begin{array}{c}0.0300 \\
(0.2300)\end{array}$ & $\begin{array}{c}-0.1400 \\
(0.1620)\end{array}$ \\
\hline $\mathrm{R}_{t-15}^{r e s i}$ & $\begin{array}{c}102.8500^{* *} \\
(175.7000)\end{array}$ & $\begin{array}{c}289.3630 \\
(280.1000)\end{array}$ & $\begin{array}{c}384.8020^{* *} \\
(187.1000)\end{array}$ & $\begin{array}{c}377.7640^{* *} \\
(195.8000)\end{array}$ & $\begin{array}{c}364.3410 \\
(297.0000)\end{array}$ \\
\hline $\mathrm{R}_{t-15}^{\text {resi }} \mathbf{1}_{\text {News }}$ & $\begin{array}{r}-249.1500 \\
(268.0000)\end{array}$ & $\begin{array}{c}-206.1300 \\
(371.27000)\end{array}$ & $\begin{array}{c}-343.4100 \\
(307.5000)\end{array}$ & $\begin{array}{r}-653.0300^{*} \\
(382.1000)\end{array}$ & $\begin{array}{c}-285.4200 \\
(379.4000)\end{array}$ \\
\hline $\mathrm{R}_{t-15}^{\text {resi }} \mathbf{1}_{\text {Post }}$ & $\begin{array}{c}130.7780 \\
(242.4000)\end{array}$ & $\begin{array}{c}231.5660 \\
(326.1000)\end{array}$ & $\begin{array}{c}191.9710 \\
(234.9000)\end{array}$ & $\begin{array}{l}-19.5200 \\
(264.7000)\end{array}$ & $\begin{array}{c}173.5970 \\
(337.7000)\end{array}$ \\
\hline $\mathrm{R}_{t-15}^{\text {resi }} \mathbf{1}_{\text {News }} \mathbf{1}_{\text {Post }}$ & $\begin{array}{c}593.8680 \\
(632.3000)\end{array}$ & $\begin{array}{c}311.2790 \\
(717.7000)\end{array}$ & $\begin{array}{c}550.4130 \\
(644.9000)\end{array}$ & $\begin{array}{c}1153.7840^{*} \\
(700.1000)\end{array}$ & $\begin{array}{c}892.0710 \\
(666.6000)\end{array}$ \\
\hline $\mathrm{X}_{t}$ & $\begin{array}{c}-0.0400 \\
(0.0500)\end{array}$ & $\begin{array}{c}0.0038 \\
(0.0045)\end{array}$ & $\begin{array}{c}0.0037 \\
(0.0028)\end{array}$ & $\begin{array}{c}-0.0003 \\
(0.0007)\end{array}$ & $\begin{array}{c}0.0012 \\
(0.0049)\end{array}$ \\
\hline $\mathrm{X}_{t} \mathbf{1}_{\text {News }}$ & $\begin{array}{l}0.5270^{* *} \\
(0.2430)\end{array}$ & $\begin{array}{c}-0.0003 \\
(0.0086)\end{array}$ & $\begin{array}{c}0.0030 \\
(0.0100)\end{array}$ & $\begin{array}{c}0.0029 \\
(0.0018)\end{array}$ & $\begin{array}{c}0.0058 \\
(0.0140)\end{array}$ \\
\hline $\mathrm{X}_{t} \mathbf{1}_{\text {Post }}$ & $\begin{array}{c}0.0420 \\
(0.1050)\end{array}$ & $\begin{array}{c}-0.0075 \\
(0.0059)\end{array}$ & $\begin{array}{c}-0.0099^{* *} \\
(0.0044)\end{array}$ & $\begin{array}{l}0.0018^{* *} \\
(0.0009)\end{array}$ & $\begin{array}{c}0.0004 \\
(0.0130)\end{array}$ \\
\hline $\mathrm{X}_{t} \mathbf{1}_{\text {News }} \mathbf{1}_{\text {Post }}$ & $\begin{array}{c}-0.3800 \\
(0.2620)\end{array}$ & $\begin{array}{c}0.0370 \\
(0.0260)\end{array}$ & $\begin{array}{c}0.0120 \\
(0.0160)\end{array}$ & $\begin{array}{c}-0.0038 \\
(0.0043)\end{array}$ & $\begin{array}{c}0.0320 \\
(0.0320)\end{array}$ \\
\hline $\operatorname{Sur}_{t} \mathbf{1}_{\text {Post }}$ & $\begin{array}{c}0.1490 \\
(0.1690)\end{array}$ & $\begin{array}{c}0.0520 \\
(0.1730)\end{array}$ & $\begin{array}{c}0.0390 \\
(0.1810)\end{array}$ & $\begin{array}{c}0.0700 \\
(0.1680)\end{array}$ & $\begin{array}{c}0.0830 \\
(0.1640)\end{array}$ \\
\hline Adjusted $\mathrm{R}^{2}$ & 0.3100 & 0.0206 & 0.0129 & 0.0105 & 0.0229 \\
\hline
\end{tabular}

announcements when I use the Relatively Important sample. Depth at the second best quote is still significant at the $1 \%$ level, but absolute order flow is significant at the $10 \%$ level. 
Overall, these results reinforce my main findings: volatility changes significantly with the arrival of macroeconomic announcements in the market. Among public information shocks and private information shocks, the latter show a significant impact on volatility. Although these alternative volatility measures lead to similar findings, the answer varies to the follow up question: which proxy for private information shocks captures this effect? Using the Realised Variance measure, the sensitivity comes through the spread. However, using the $A R$ measure, spread and absolute order flow are the key measures. Using the $R^{\text {resi }}$ measure, depth at the second best quote and absolute order flow are the proxies that capture the effect. The results confirm my main assertion that volatility is related to liquidity measures that are a proxy for private information shocks. However, the main results (using the Realised Variance measure) are most relevant. The reason for this is that when using the Realised Variance measure, I compute the returns at the best quotes for each one-minute interval and then aggregate the squared returns, while when using the $A R$ and $R^{r e s i}$ measures, I only look at the beginning and the end of 15-minute intervals. In other words, these measures miss the impact of quotes within the 15-minute interval, which, in high frequency data, have significant impacts. Hence I would advocate the use of spread as a measure for private information over absolute order imbalance or depth.

\subsubsection{The Effect of All Liquidity Variables Jointly}

In this section, I examine the effect of all liquidity measures jointly. So far, I have examined three different measures of volatility along with three different samples of macroeconomic announcements. I now implement the same regression model using (2.9) with the Realised Variance measure of volatility along with the three different samples. However, I now include all the liquidity measures. Spread at the best quote $\left(S_{0}\right)$ and depth at the second best quote $\left(D_{1}\right)$ are the main microstructure measures that are sig- 
nificant when I use the Realised Variance measure along with the Full and MI samples. Table 2.21 reports my results.

Table 2.21 shows that when I examine the effect of all liquidity measures jointly using the Realised Variance measure, spread and depth at the second best quote are the microstructure variables that exhibit greater impact on Realised Variance after an announcement. When I use the Full sample the results are significant only for spread at the $1 \%$ level. However, when I use the Most Important sample, the results are significant for both spread and depth at the second best quote at the 10\% level. The Relatively Important sample does not reveal any significant results. Surprise, as a measure of public information shocks, is insignificant for all my samples. These results are broadly consistent with my analysis in Section 2.3.2 when I use liquidity measures individually. Again, I note that rises in spread or depth at the second best quote are symptoms of illiquidity. The main distinction between the two sets of results is the finding of a private information shock using the Most Important sample.

Overall, the results show that when I test the effect of all the liquidity measures jointly, spread at the best quote $\left(S_{0}\right)$ is the main explanatory variable that captures the private information shock effect. Depth at the second best quote $\left(D_{1}\right)$ is another liquidity measure that has a significantly stronger relationship with volatility after the arrival of macroeconomic announcements but only in the Most Important sample (at the $10 \%$ level). Overall, with the arrival of macroeconomic announcements, as noted earlier, private information shocks are the main source of volatility increases in the market.

\subsubsection{The Effect of News at Times Other Than at 8:30}

So far, I have only examined the price changes in the Canadian FX market when there is an 8:30 announcement. A question arising here is: what if I 
TABLE 2.21: The Effect of All Liquidity Variables Jointly on Realised Variance.

Results of regression of dependent variable ( $R V$ ) realised variance on liquidity measures: $S_{0}$ is spread, $D_{0}$ is the depth at the best quote, $D_{1}$ is the depth at the second best quote, $A O F$ is the absolute order flow, AOI is the absolute order imbalance. Descriptions of the calculation of the variables can be found in Section 2.3. There are 330 observations in full announcements sample. The superscripts ***,**, and * indicate significance at the 1\%,5\%, and 10\% level respectively. The significance is assessed using Newey-West standard errors.

\begin{tabular}{|c|c|c|c|c|c|c|c|}
\hline \multirow{2}{*}{$\begin{array}{l}\text { Explanatory } \\
\text { Variables }\end{array}$} & \multicolumn{3}{|c|}{ Samples } & \multirow{2}{*}{$\begin{array}{l}\text { Explanatory } \\
\text { - Variables }\end{array}$} & \multicolumn{3}{|c|}{ Samples } \\
\hline & Full & RI & MI & & Full & RI & MI \\
\hline Intercept & $\begin{array}{l}0.0960^{* * *} \\
(0.0315)\end{array}$ & $\begin{array}{c}0.0960^{* * *} \\
(0.0321)\end{array}$ & $\begin{array}{l}0.0960^{* * *} \\
(0.0345)\end{array}$ & $\mathrm{D}_{1, t}$ & $\begin{array}{c}-0.0017 \\
(0.0017)\end{array}$ & $\begin{array}{r}-0.0017 \\
(0.0017)\end{array}$ & $\begin{array}{r}-0.0017 \\
(0.0018)\end{array}$ \\
\hline $\mathbf{1}_{\text {News }}$ & $\begin{array}{c}0.0153 \\
(0.0427)\end{array}$ & $\begin{array}{c}0.0148 \\
(0.0471)\end{array}$ & $\begin{array}{c}0.0131 \\
(0.0670)\end{array}$ & $\mathrm{D}_{1, t} \mathbf{1}_{\text {News }}$ & $\begin{array}{c}0.0016 \\
(0.0023)\end{array}$ & $\begin{array}{c}0.0000 \\
(0.0024)\end{array}$ & $\begin{array}{r}-0.0003 \\
(0.0048)\end{array}$ \\
\hline $\mathbf{1}_{\text {Post }}$ & $\begin{array}{c}-0.0622 \\
(0.0518)\end{array}$ & $\begin{array}{c}-0.0622 \\
(0.0528)\end{array}$ & $\begin{array}{c}-0.0622 \\
(0.0568)\end{array}$ & $\mathrm{D}_{1, t} \mathbf{1}_{\text {Post }}$ & $\begin{array}{c}0.0012 \\
(0.0025)\end{array}$ & $\begin{array}{c}0.0012 \\
(0.0026)\end{array}$ & $\begin{array}{c}0.0012 \\
(0.0028)\end{array}$ \\
\hline $\mathbf{1}_{\text {News }} \mathbf{1}_{\text {Post }}$ & $\begin{array}{c}0.0853 \\
(0.0895)\end{array}$ & $\begin{array}{c}-0.0400 \\
(0.1041)\end{array}$ & $\begin{array}{c}-0.2323 \\
(0.1607)\end{array}$ & $\mathrm{D}_{1, t} \mathbf{1}_{\text {News }} \mathbf{1}_{\text {Post }}$ & $\begin{array}{c}0.0008 \\
(0.0063)\end{array}$ & $\begin{array}{c}0.0054 \\
(0.0081)\end{array}$ & $\begin{array}{r}0.0163^{*} \\
(0.0088)\end{array}$ \\
\hline $\mathrm{R}_{t-15}$ & $\begin{array}{l}0.3356^{* * *} \\
(0.0590)\end{array}$ & $\begin{array}{l}0.3356^{* * *} \\
(0.0601)\end{array}$ & $\begin{array}{l}0.3356^{* * *} \\
(0.0647)\end{array}$ & $\mathrm{AOF}_{t}$ & $\begin{array}{c}0.0002 \\
(0.0002)\end{array}$ & $\begin{array}{c}0.0002 \\
(0.0002)\end{array}$ & $\begin{array}{c}0.0002 \\
(0.0003)\end{array}$ \\
\hline $\mathrm{R}_{t-15} \mathbf{1}_{\text {News }}$ & $\begin{array}{c}-0.1257 \\
(0.1901)\end{array}$ & $\begin{array}{c}0.0086 \\
(0.2394)\end{array}$ & $\begin{array}{c}-0.3805 \\
(0.2436)\end{array}$ & $\mathrm{AOF}_{t} \mathbf{1}_{\text {News }}$ & $\begin{array}{c}-0.0002 \\
(0.0003)\end{array}$ & $\begin{array}{r}-0.0002 \\
(0.0003)\end{array}$ & $\begin{array}{c}0.0011 \\
(0.0007)\end{array}$ \\
\hline $\mathbf{R}_{t-15} \mathbf{1}_{\text {Post }}$ & $\begin{array}{c}0.1431 \\
(0.1568)\end{array}$ & $\begin{array}{c}0.1431 \\
(0.1597)\end{array}$ & $\begin{array}{c}0.1431 \\
(0.1719)\end{array}$ & $\mathrm{AOF}_{t} \mathbf{1}_{\text {Post }}$ & $\begin{array}{c}0.0001 \\
(0.0004)\end{array}$ & $\begin{array}{c}0.0001 \\
(0.0004)\end{array}$ & $\begin{array}{c}0.0001 \\
(0.0004)\end{array}$ \\
\hline $\mathrm{R}_{t-15} \mathbf{1}_{\text {News }} \mathbf{1}_{\text {Post }}$ & $\begin{array}{c}0.3092 \\
(0.3822)\end{array}$ & $\begin{array}{c}-0.1574 \\
(0.4677)\end{array}$ & $\begin{array}{c}2.9951 \\
(1.9775)\end{array}$ & $\mathrm{AOF}_{t} \mathbf{1}_{\text {News }} \mathbf{1}_{\text {Post }}$ & $\begin{array}{c}-0.0001 \\
(0.0005)\end{array}$ & $\begin{array}{c}0.0006 \\
(0.0008)\end{array}$ & $\begin{array}{c}0.0021 \\
(0.0040)\end{array}$ \\
\hline $\mathrm{S}_{0, t}$ & $\begin{array}{c}0.0182 \\
(0.0469)\end{array}$ & $\begin{array}{c}0.0182 \\
(0.0478)\end{array}$ & $\begin{array}{c}0.0182 \\
(0.0514)\end{array}$ & $\mathrm{AOI}_{t}$ & $\begin{array}{c}0.0064 \\
(0.0051)\end{array}$ & $\begin{array}{c}0.0064 \\
(0.0052)\end{array}$ & $\begin{array}{c}0.0064 \\
(0.0055)\end{array}$ \\
\hline $\mathrm{S}_{0, t} \mathbf{1}_{\text {News }}$ & $\begin{array}{c}-0.0610 \\
(0.0750)\end{array}$ & $\begin{array}{r}-0.0187 \\
(0.0844)\end{array}$ & $\begin{array}{c}-0.1541 \\
(0.1263)\end{array}$ & $\mathrm{AOI}_{t} \mathbf{1}_{N e w s}$ & $\begin{array}{c}-0.0097 \\
(0.0070)\end{array}$ & $\begin{array}{c}-0.0084 \\
(0.0075)\end{array}$ & $\begin{array}{r}-0.0110 \\
(0.0104)\end{array}$ \\
\hline $\mathrm{S}_{0, t} \mathbf{1}_{\text {Post }}$ & $\begin{array}{c}0.0401 \\
(0.0780)\end{array}$ & $\begin{array}{c}0.0401 \\
(0.0794)\end{array}$ & $\begin{array}{c}0.0401 \\
(0.0855)\end{array}$ & $\mathrm{AOI}_{t} \mathbf{1}_{\text {Post }}$ & $\begin{array}{c}-0.0143 \\
(0.0095)\end{array}$ & $\begin{array}{c}-0.0143 \\
(0.0097)\end{array}$ & $\begin{array}{r}-0.0143 \\
(0.0104)\end{array}$ \\
\hline $\mathrm{S}_{0, t} \mathbf{1}_{\text {News }} \mathbf{1}_{\text {Post }}$ & $\begin{array}{l}0.2664^{* * *} \\
(0.1157)\end{array}$ & $\begin{array}{c}0.1591 \\
(0.1142)\end{array}$ & $\begin{array}{c}0.2520^{*} \\
(0.1525)\end{array}$ & $\mathrm{AOI}_{t} \mathbf{1}_{\text {News }} \mathbf{1}_{\text {Post }}$ & $\begin{array}{c}0.0166 \\
(0.0162)\end{array}$ & $\begin{array}{c}-0.0109 \\
(0.0263)\end{array}$ & $\begin{array}{r}-0.0464 \\
(0.0501)\end{array}$ \\
\hline $\mathrm{D}_{0, t}$ & $\begin{array}{c}-0.0065 \\
(0.0045)\end{array}$ & $\begin{array}{c}-0.0065 \\
(0.0046)\end{array}$ & $\begin{array}{c}-0.0065 \\
(0.0049)\end{array}$ & $\operatorname{Sur}_{t} \mathbf{1}_{\text {Post }}$ & $\begin{array}{c}-0.0070 \\
(0.0220)\end{array}$ & $\begin{array}{c}0.0072 \\
(0.0267)\end{array}$ & $\begin{array}{r}-0.1508 \\
(0.1226)\end{array}$ \\
\hline $\mathrm{D}_{0, t} \mathbf{1}_{\text {News }}$ & $\begin{array}{c}0.0069 \\
(0.0054)\end{array}$ & $\begin{array}{c}0.0056 \\
(0.0058)\end{array}$ & $\begin{array}{c}0.0082 \\
(0.0067)\end{array}$ & Adjusted $\mathrm{R}^{2}$ & 0.2688 & 0.3337 & 0.5597 \\
\hline $\mathrm{D}_{0, t} \mathbf{1}_{\text {Post }}$ & $\begin{array}{c}0.0100 \\
(0.0073)\end{array}$ & $\begin{array}{c}0.0100 \\
(0.0074)\end{array}$ & $\begin{array}{c}0.0100 \\
(0.0080)\end{array}$ & & & & \\
\hline $\mathrm{D}_{0, t} \mathbf{1}_{\text {News }} \mathbf{1}_{\text {Post }}$ & $\begin{array}{c}-0.0184 \\
(0.0138)\end{array}$ & $\begin{array}{c}0.0140 \\
(0.0252)\end{array}$ & $\begin{array}{c}0.0416 \\
(0.0526)\end{array}$ & & & & \\
\hline
\end{tabular}


include other announcements outside the 8:30 interval? Does this assumption change my results, or can I still assert that it is the arrival of private information that affects volatility?

The pre-scheduled macroeconomic announcements are released at different times. The list of all news and their arrival times are reported in Tables 2.1 and 2.2. According to the reported list, a majority of news releases are at 8:30 and the rest are at different times. Depending on the country and the nature of the macroeconomic indicator, I have news at 7:00, 8:30, 9:15, 10:00, and 14:15. To examine the effect of news as a whole, I include all the announcements outside the 8:30 interval (as well as the 8:30 announcements). As noted previously in Section 2.2.2, I remove overlapping announcements. I set up my regression model using equation (2.9) to examine realised variance as my main volatility measure through the Full, Relatively Important, and Most Important samples. Table 2.22 shows the results of using the Realised Variance measure for three different samples when I examine the impact of announcements at times other than 8:30. The results of using the Realised Variance measure for the Full sample reveals significant results for spread at the best quote $\left(S_{0}\right)$, depth at the best quote $\left(D_{0}\right)$, and absolute order imbalance $(A O I)$. The spread at the best quote is significant at the $1 \%$ level while depth at the best quote and absolute order imbalance are significant at the 5\% level. The Relatively Important sample reveals significant results for spread at the best quote $\left(S_{0}\right)$ and depth at the second best quote $\left(D_{1}\right)$ at the $10 \%$ and $5 \%$ level respectively. The Most Important sample shows significant results for depth at the best quote $\left(D_{0}\right)$ at the $10 \%$ level.

Overall, these results are consistent with my findings for the 8:30 announcement using the full sample. The effect of private information shocks appear in spread at the best quote, depth at the best quote, and absolute order imbalance for the Full sample but for the Relatively Important samples it comes through the spread at the best quote and depth behind the best 
TABLE 2.22: The Effect of News at Times other than 8:30 on Realised Variance.

Results of regression of dependent variable $(R V)$ realised variance on liquidity measures: $S_{0}$ is spread, $D_{0}$ is the depth at the best quote, $D_{1}$ is the depth at the second best quote, AOF is the absolute order flow, AOI is the absolute order imbalance. Descriptions of the calculation of the variables can be found in Section 2.3. There are 330 observations in full announcements sample. The superscripts ${ }^{* *}, * *$, and ${ }^{*}$ indicate significance at the $1 \%, 5 \%$, and $10 \%$ level respectively. The significance is assessed using Newey-West standard errors.

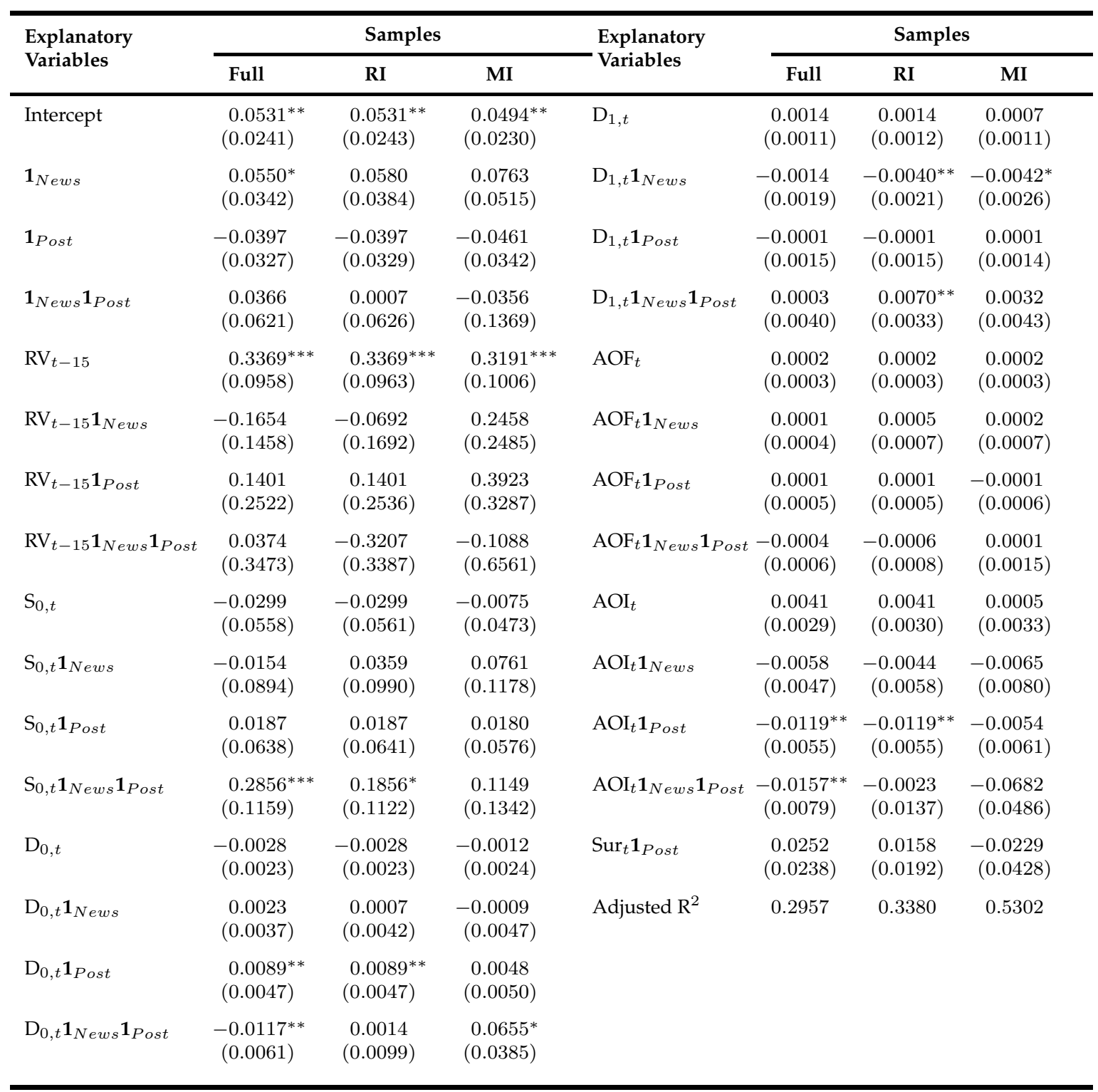


quote. The Most Important sample has significant results only for depth at the best quote. I reiterate that rises in spread, rises in the depth at the second best quote, falls in the depth at the best quote, or rises in the absolute order imbalance, are all signs of illiquidity. The only significant coefficient that is contrary to this is the effect of depth at the best quote with the Most Important sample. Here, a rise in liquidity seems to lead to greater volatility. I conclude that inclusion of other announcement times does not materially change my findings. If anything, I obtain stronger results from this enlarged sample.

\subsubsection{The Magnitude of Liquidity Variable Changes}

I have shown that the sensitivity of volatility to liquidity measures changes in response to macroeconomic news. I now turn my attention to the level of my liquidity measures: are they large enough to make this sensitivity change economically significant? In this regard, I examine the difference between news days and no-news days during the 15-minute interval before and after 8:30. To have a closer look, I split each 15-minute interval before and after 8:30 into three 5-minute intervals.

Table 2.23 shows that the main liquidity measures with significant results are the bid-ask spread at the most competitive quote $\left(S_{0}\right)$, absolute order flow $(A O F)$, and absolute order imbalance $(A O I)$. My sensitivity regressions (see Sections 2.3.2 and 2.4.6) show that volatility has a heightened sensitivity to $S_{0}$ and $A O I$ following announcements (but not $A O F$ ), so I focus my attention on these variables. The difference between news days and no-news days for spread has a large and significant increase in the first 5-minute interval after the arrival of macroeconomic announcements. This difference becomes larger as I move from the full sample to the Most Important sample. The absolute order imbalance also shows an increase in difference between news days and no-news days. However, 
TABLE 2.23: The Magnitude of Changes after Announcements.

Each number represents the average difference in the liquidity measure, at the indicated 5-minute window, between news days and no-news days. My Liquidity measures are: $S_{0}$ is spread, $D_{0}$ is the depth at the best quote, $D_{1}$ is the depth at the second best quote, $A O F$ is the absolute order flow, AOI is the absolute order imbalance. Descriptions of the calculation of the measures can be found in Section 2.3. There are 330 observations in full announcements sample. The superscripts ${ }_{* * *}^{* * *}$, and * indicate significance at the $1 \%, 5 \%$, and $10 \%$ level respectively. The significance is assessed using Newey-West standard errors.

\begin{tabular}{|c|c|c|c|c|c|c|c|c|c|}
\hline \multirow{2}{*}{ Interval } & Full & RI & MI & Full & RI & MI & Full & RI & MI \\
\hline & \multicolumn{3}{|c|}{$S_{0}$} & \multicolumn{3}{|c|}{$D_{0}$} & \multicolumn{3}{|c|}{$D_{1}$} \\
\hline-3 & $\begin{array}{c}0.0291 \\
(0.0297)\end{array}$ & $\begin{array}{c}0.0437 \\
(0.0317)\end{array}$ & $\begin{array}{c}-0.0075 \\
(0.0344)\end{array}$ & $\begin{array}{c}-0.2146 \\
(0.8399)\end{array}$ & $\begin{array}{c}-0.3136 \\
(0.8962)\end{array}$ & $\begin{array}{c}-0.0041 \\
(1.2749)\end{array}$ & $\begin{array}{c}-0.3267 \\
(1.0221)\end{array}$ & $\begin{array}{c}-0.2380 \\
(1.0760)\end{array}$ & $\begin{array}{c}0.0827 \\
(1.1179)\end{array}$ \\
\hline-2 & $\begin{array}{c}-0.0228 \\
(0.0342)\end{array}$ & $\begin{array}{c}-0.0265 \\
(0.0328)\end{array}$ & $\begin{array}{c}0.0176 \\
(0.0546)\end{array}$ & $\begin{array}{c}-0.1194 \\
(1.0828)\end{array}$ & $\begin{array}{c}0.1037 \\
(1.3666)\end{array}$ & $\begin{array}{c}-1.6457 \\
(0.9916)\end{array}$ & $\begin{array}{c}-2.2088 \\
(2.5917)\end{array}$ & $\begin{array}{c}-3.0326 \\
(2.5938)\end{array}$ & $\begin{array}{c}-3.2965 \\
(2.9457)\end{array}$ \\
\hline-1 & $\begin{array}{c}-0.0141 \\
(0.0259)\end{array}$ & $\begin{array}{c}-0.0166 \\
(0.0291)\end{array}$ & $\begin{array}{c}-0.0501 \\
(0.0350)\end{array}$ & $\begin{array}{c}-0.0944 \\
(0.5835)\end{array}$ & $\begin{array}{c}0.4205 \\
(0.6963)\end{array}$ & $\begin{array}{c}1.3004 \\
(1.1246)\end{array}$ & $\begin{array}{c}-0.8729 \\
(0.8213)\end{array}$ & $\begin{array}{c}-0.5112 \\
(0.9649)\end{array}$ & $\begin{array}{c}0.1271 \\
(1.8823)\end{array}$ \\
\hline+1 & $\begin{array}{c}0.1694^{* *} \\
(0.0534)\end{array}$ & $\begin{array}{r}* 0.2204^{* *} \\
(0.0789)\end{array}$ & $\begin{array}{c}0.5633^{* *} \\
(0.2563)\end{array}$ & $\begin{array}{c}-1.0817 \\
(1.1787)\end{array}$ & $\begin{array}{c}-0.9523 \\
(1.2520)\end{array}$ & $\begin{array}{c}-0.9589 \\
(1.4077)\end{array}$ & $\begin{array}{c}-0.2982 \\
(0.8469)\end{array}$ & $\begin{array}{c}-0.5634 \\
(0.8731)\end{array}$ & $\begin{array}{c}0.9474 \\
(1.8377)\end{array}$ \\
\hline+2 & $\begin{array}{c}0.0046 \\
(0.0251)\end{array}$ & $\begin{array}{c}-0.0098 \\
(0.0264)\end{array}$ & $\begin{array}{c}-0.0208 \\
(0.0329)\end{array}$ & $\begin{array}{c}-1.4324 \\
(1.4172)\end{array}$ & $\begin{array}{c}-1.4651 \\
(1.4659)\end{array}$ & $\begin{array}{r}-2.7920 \\
(1.4476)\end{array}$ & $\begin{array}{c}1.7437 \\
(1.4175)\end{array}$ & $\begin{array}{c}3.0385 \\
(1.9906)\end{array}$ & $\begin{array}{c}2.6560 \\
(1.5554)\end{array}$ \\
\hline+3 & $\begin{array}{c}0.0069 \\
(0.0251)\end{array}$ & $\begin{array}{c}0.0116 \\
(0.0264)\end{array}$ & $\begin{array}{c}0.0513 \\
(0.0329)\end{array}$ & $\begin{array}{c}0.5272 \\
(0.7913)\end{array}$ & $\begin{array}{c}0.5447 \\
(0.8621)\end{array}$ & $\begin{array}{c}0.4780 \\
(0.9089)\end{array}$ & $\begin{array}{c}0.2548 \\
(0.9592)\end{array}$ & $\begin{array}{c}0.7724 \\
(1.2072)\end{array}$ & $\begin{array}{c}-0.4609 \\
(1.5567)\end{array}$ \\
\hline Interval & \multicolumn{3}{|c|}{$A O F$} & \multicolumn{3}{|c|}{$A O I$} & & & \\
\hline-3 & $\begin{array}{l}17.9241^{* *} \\
(5.8585)\end{array}$ & $\begin{array}{c}* 4.4140 \\
(4.2504)\end{array}$ & $\begin{array}{c}-3.6583 \\
(3.8985)\end{array}$ & $\begin{array}{c}-5.1930^{* *} \\
(0.6511)\end{array}$ & $\begin{array}{r}* 0.5847 \\
(0.6878)\end{array}$ & $\begin{array}{c}-0.2561 \\
(0.8449)\end{array}$ & & & \\
\hline-2 & $\begin{array}{l}20.0236^{* *} \\
(9.4313)\end{array}$ & $\begin{array}{c}2.4444 \\
(5.8015)\end{array}$ & $\begin{array}{c}-7.5893 \\
(5.2297)\end{array}$ & $\begin{array}{c}0.3894 \\
(0.8274)\end{array}$ & $\begin{array}{c}0.5826 \\
(1.0945)\end{array}$ & $\begin{array}{c}-0.5931 \\
(0.7062)\end{array}$ & & & \\
\hline-1 & $\begin{array}{l}26.9598^{* *} \\
(4.5272)\end{array}$ & $\begin{array}{l}* \quad 6.9910^{* *} \\
(3.0745)\end{array}$ & $\begin{array}{c}-0.7345 \\
(3.0789)\end{array}$ & $\begin{array}{c}-0.1183 \\
(0.4995)\end{array}$ & $\begin{array}{c}0.1731 \\
(0.5434)\end{array}$ & $\begin{array}{c}1.5571 \\
(1.0266)\end{array}$ & & & \\
\hline+1 & $\begin{array}{l}27.3048^{* *} \\
(4.9454)\end{array}$ & $\begin{array}{c}* 9.6451^{* *} \\
(2.5282)\end{array}$ & $\begin{array}{c}0.3968 \\
(2.3868)\end{array}$ & $\begin{array}{c}-0.1183 \\
(0.8454)\end{array}$ & $\begin{array}{c}0.1731 \\
(0.9551)\end{array}$ & $\begin{array}{c}1.5571 \\
(1.0839)\end{array}$ & & & \\
\hline+2 & $\begin{array}{l}44.1696^{* *} \\
(7.0332)\end{array}$ & $\begin{array}{c}{ }^{*} 20.0446^{* *} \\
(4.1386)\end{array}$ & $\begin{array}{l}16.6754^{* *} \\
(6.6238)\end{array}$ & $\begin{array}{c}-0.9489 \\
(1.1922)\end{array}$ & $\begin{array}{c}-0.6352 \\
(1.2472)\end{array}$ & $\begin{array}{c}-1.6418 \\
(1.2236)\end{array}$ & & & \\
\hline+3 & $\begin{array}{c}34.4161^{* *} \\
(8.0706)\end{array}$ & $\begin{array}{c}{ }^{*} 14.4881^{* *} \\
(6.0167)\end{array}$ & $\begin{array}{c}8.6071 \\
(7.5121)\end{array}$ & $\begin{array}{c}0.2948^{* *} \\
(0.0297)\end{array}$ & $\begin{array}{c}0.1545 \\
(0.7638)\end{array}$ & $\begin{array}{c}-0.2122 \\
(0.8599)\end{array}$ & & & \\
\hline
\end{tabular}


the large increase happens in the third 5-minute interval after the arrival of news. This liquidity measure does not follow a specific pattern compared to spread. Overall, my conclusion is that volatility not only shows a heightened sensitivity to spread following the arrival of news but also spread has (on average) a significant increase, which makes this sensitivity important. This pattern is not so clear and significant for other liquidity measures. This reinforces my conclusion from my main analyses that spread is the most important microstructure measure for capturing private information.

\subsubsection{Risk Averse/Seeking Trader in the Market}

In this part, I examine the market condition with different level of private information for a risk averse/seeking trader. I calculate the returns, the standard deviation of returns, and spread in each one-minute over the 15-minute interval after the 8:30 on news days and no-news days. The spread median is used as a threshold to determine low/high private information level. I evaluate the market condition for a risk averse/seeking trader using the Full, Relatively Important, and Most Important samples. Table 2.24 presents the results for the Canadian FX market in year 2005. Panel A, shows the Full sample with 122 observations on news days and $43 \mathrm{ob}-$ servations on no-news days. On average, returns and standard deviation of returns increase from $0.71 \%$ and $11.61 \%$ to $0.81 \%$ and $14.01 \%$ respectively on news days as the level of private information goes up. Similarly, on average, returns and standard deviation of returns increase from $-0.11 \%$ and $7.44 \%$ to $0.13 \%$ and $7.53 \%$ respectively on no-news days as the level of private information increases. Results show that a higher level of private information (more than the spread median) provides higher return with higher standard deviation and a lower level of private information (less than the spread median) provides less return with less standard deviation. 
Panel B presents the Relatively Important news with 81 and 42 observations on news days and no-news days respectively. On average, returns and standard deviation of returns increase from $-0.13 \%$ and $12.65 \%$ to $0.74 \%$ and $13.91 \%$ respectively on news days as the level of private information goes up. Similarly, on average, returns and standard deviation of returns increase from $-0.65 \%$ and $8.01 \%$ to $0.82 \%$ and $7.89 \%$ respectively on no-news days as the level of private information increases. Finally, Panel C presents the Most Important news with 54 and 42 observations on news days and no-news days respectively. On average, returns and standard deviation of returns increase from $0.09 \%$ and $14.14 \%$ to $1.74 \%$ and $14.92 \%$ respectively on news days as the level of private information goes up. Similarly, on average, returns and standard deviation of returns increase from $-0.65 \%$ and $8.01 \%$ to $0.82 \%$ and $7.89 \%$ respectively on no-news days as the level of private information increases. I use the t-test to evaluate whether there is a significant difference between high and low sample on news days and no-news days as well as across groups. Results show no significant results for any of comparisons that might be due to small sample and having close returns on average.

Overall, a risk averse trader might trade on days with less level of private information and a risk seeking trader might trade on days with higher level of private information. Results hold the same across all three samples in Panels A, B and C on news days and no-news days.

\subsubsection{Realised Variance in Economic Samples}

In this section, I split all the macroeconomic announcements (i.e. Tables 2.1 and 2.2) into 4 different samples based on their economic function: output, prices, employment, and rate decision. The time of announcements varies within each sample and includes 8:30 and times other than it. Table 2.25 presents all pre-scheduled macroeconomic announcements in US. Simi- 
TABle 2.24: Market Condition for Risk Averse/Seeking Trader.

This table presents the return, its standard deviation, and spread for each one-minute over a 15-minute interval after 8:30 on news days and no-news days. The median of spread is used as a threshold to determine low/high level of private information in the market. Panels $A, B$, and $C$ include 122, 81, and 54 observations on news days and 43, 42, and 42 no-news days respectively.

\begin{tabular}{|c|c|c|c|c|}
\hline \multirow{2}{*}{ Sample } & \multicolumn{2}{|c|}{ News Days Information } & \multicolumn{2}{|c|}{ No-News Days Information } \\
\hline & Low & High & Low & High \\
\hline \multicolumn{5}{|c|}{ Panel A: Full Sample } \\
\hline Avg_Return & 0.0071 & 0.0081 & -0.0011 & 0.0013 \\
\hline Min_Return & -0.0938 & -0.1301 & -0.0580 & -0.0347 \\
\hline Max_Return & 0.1124 & 0.1137 & 0.0380 & 0.0405 \\
\hline Avg_std & 0.1161 & 0.1401 & 0.0744 & 0.0753 \\
\hline Min_std & 0.0000 & 0.0000 & 0.0000 & 0.0302 \\
\hline Max_std & 0.3105 & 0.3907 & 0.1287 & 0.1306 \\
\hline \multicolumn{5}{|c|}{ Panel B: Relatively Important Sample } \\
\hline Avg_Return & -0.0013 & 0.0074 & -0.0065 & 0.0082 \\
\hline Min_Return & -0.0938 & 0.0000 & 0.0322 & -0.0297 \\
\hline Max_Return & 0.1124 & 0.3067 & 0.0380 & 0.0405 \\
\hline Avg_std & 0.1265 & 0.1391 & 0.0801 & 0.0789 \\
\hline Min_std & 0.0000 & 0.0000 & 0.0322 & 0.0302 \\
\hline Max_std & 0.3229 & 0.3067 & 0.1028 & 0.1306 \\
\hline \multicolumn{5}{|c|}{ Panel C: Most Important Sample } \\
\hline Avg_Return & 0.0009 & 0.0147 & -0.0065 & 0.0082 \\
\hline Min_Return & -0.0752 & -0.1301 & -0.0580 & -0.0297 \\
\hline Max_Return & 0.0757 & 0.1137 & 0.0380 & 0.0405 \\
\hline Avg_std & 0.1414 & 0.1492 & 0.0801 & 0.0789 \\
\hline Min_std & 0.0000 & 0.0000 & 0.0322 & 0.0302 \\
\hline Max_std & 0.3229 & 0.3067 & 0.1028 & 0.1306 \\
\hline
\end{tabular}


TABLE 2.25: Pre-scheduled Macroeconomic Announcements Survey in 4 Groups in US.

This table provides summary statistics for macroeconomic announcements according to different economic groups in the US in the year 2005.

\begin{tabular}{|c|c|c|c|c|c|c|c|}
\hline Event & Source & Obs. & Time & Min & $\operatorname{Max}$ & Mean & Std. \\
\hline \multicolumn{8}{|l|}{ Panel A: Output } \\
\hline Bloomberg Consumer Confidence & B & 10 & $17: 00$ & -22 & -8 & -14.5 & 4.7668 \\
\hline Building Permits & $\mathrm{CB}$ & 12 & $08: 30$ & 1985 & $2160 \mathrm{~K}$ & $2077.9 \mathrm{~K}$ & $49.8 \mathrm{~K}$ \\
\hline Business Inventories & $\mathrm{CB}$ & 12 & $08: 30$ & 0.0010 & 0.0090 & 0.0038 & 0.0024 \\
\hline Capacity Utilization & FR & 12 & 09:15 & 0.7890 & 0.8030 & 0.7953 & 0.0035 \\
\hline Chicago Purchasing Manager & CBB & 12 & 10:00 & 52 & 62.500 & 58.617 & 3.289 \\
\hline Conf. Board Consumer Confidence & $\mathrm{CB}$ & 12 & $10: 00$ & 88 & 106.30 & 99.050 & 5.706 \\
\hline Construction Spending MoM & $\mathrm{CB}$ & 12 & $10: 00$ & 0.0030 & 0.0070 & 0.0049 & 0.0011 \\
\hline Consumer Credit & FR & 12 & $15: 00$ & $4.1 \mathrm{~B}$ & $10 \mathrm{~B}$ & $6.4 \mathrm{~B}$ & $1.6 \mathrm{~B}$ \\
\hline Durable Goods Orders & $\mathrm{CB}$ & 12 & $08: 30$ & -0.0150 & 0.0150 & 0.0034 & 0.0111 \\
\hline Empire Manufacturing & FR & 12 & $08: 30$ & 1 & 25 & 15.983 & 6.1573 \\
\hline Factory Orders & $\mathrm{CB}$ & 12 & $10: 00$ & -0.0230 & 0.0300 & 0.0058 & 0.0153 \\
\hline GDP Annualised QoQ & BEA & 12 & $08: 30$ & 0.0330 & 0.0430 & 0.0368 & 0.0029 \\
\hline Housing Starts & $\mathrm{CB}$ & 12 & $08: 30$ & $1903 K$ & $2090 \mathrm{~K}$ & 2013.6K & $56.2 \mathrm{~K}$ \\
\hline ISM Manufacturing & ISM & 12 & $10: 00$ & 51.400 & 58.500 & 55.383 & 2.4778 \\
\hline Leading Index & $\mathrm{CB}$ & 12 & $10: 00$ & -0.0050 & 0.0080 & 0.0003 & 0.0040 \\
\hline Monthly Budget Statement & $\mathrm{T}$ & 12 & $14: 00$ & $-100 \mathrm{~B}$ & $60 \mathrm{~B}$ & $-26.9 B$ & $50.8 \mathrm{~B}$ \\
\hline NAHB Housing Market Index & NAHB & 12 & $13: 00$ & 61 & 71 & 67.833 & 2.9797 \\
\hline New Home Sales & $\mathrm{CB}$ & 12 & 10:00 & $1125 \mathrm{~K}$ & $1350 \mathrm{~K}$ & $1253.4 \mathrm{~K}$ & $77.5 \mathrm{~K}$ \\
\hline Personal Consumption & BEA & 10 & $08: 30$ & 0.0300 & 0.0430 & 0.0359 & 0.0042 \\
\hline Personal Income & BEA & 12 & $08: 30$ & -0.0260 & 0.0340 & 0.0041 & 0.0128 \\
\hline Personal Spending & BEA & 12 & $08: 30$ & -0.0020 & 0.0100 & 0.0045 & 0.0036 \\
\hline Philadelphia Fed Business Outlook & PFR & 12 & $12: 00$ & 10 & 25 & 14.733 & 4.6582 \\
\hline Retail Sales Advance MoM & $\mathrm{CB}$ & 12 & $08: 30$ & -0.0140 & 0.0210 & 0.0038 & 0.0093 \\
\hline $\begin{array}{l}\text { Trade Balance } \\
\text { Wholesale Inventories MoM }\end{array}$ & $\begin{array}{l}\mathrm{CB} \\
\mathrm{CB}\end{array}$ & $\begin{array}{l}12 \\
12\end{array}$ & $\begin{array}{l}09: 30 \\
10: 00\end{array}$ & $\begin{array}{l}-62.9 \mathrm{~B} \\
0.0030\end{array}$ & $\begin{array}{l}-54 \mathrm{~B} \\
0.0090\end{array}$ & $\begin{array}{l}-58.7 \mathrm{~B} \\
0.0056\end{array}$ & $\begin{array}{l}2.6 \mathrm{~B} \\
0.0017\end{array}$ \\
\hline \multicolumn{8}{|l|}{ Panel B: Prices } \\
\hline CPI MoM & BLS & 12 & $08: 30$ & -0.0040 & 0.0090 & 0.0025 & 0.0033 \\
\hline Current Account Balance & BEA & 4 & $08: 30$ & $-205 B$ & $-183 B$ & $-192.75 B$ & $9.2 \mathrm{~B}$ \\
\hline Import Price Index MoM & BLS & 12 & $08: 30$ & -0.0050 & 0.0140 & 0.0047 & 0.0068 \\
\hline Industrial Production Price MoM & FR & 12 & 09:15 & -0.004 & 0.01 & 0.0034 & 0.0031 \\
\hline PCE Core YoY & BEA & 11 & $08: 30$ & 0.0150 & 0.0190 & 0.0174 & 0.0016 \\
\hline \multicolumn{8}{|l|}{ Panel C: Employment } \\
\hline Change in Nonfarm Payrolls & BLS & 12 & $08: 30$ & $-150 K$ & $225 \mathrm{~K}$ & $159.3 \mathrm{~K}$ & $101.1 \mathrm{~K}$ \\
\hline Continuing Claims & DL & 50 & $08: 30$ & $2573 K$ & $2939 K$ & $2664.7 \mathrm{~K}$ & $93.2 \mathrm{~K}$ \\
\hline Employment Cost Index & BLS & 4 & $08: 30$ & 0.0080 & 0.0100 & 0.0085 & 0.0010 \\
\hline Initial Jobless Claims & DL & 52 & $08: 30$ & $309 \mathrm{~K}$ & $450 \mathrm{~K}$ & $329.8 \mathrm{~K}$ & $24.5 \mathrm{~K}$ \\
\hline Nonfarm Productivity & BLS & 8 & $08: 30$ & 0.0150 & 0.0450 & 0.0238 & 0.0100 \\
\hline Unemployment Rate & BLS & 12 & $08: 30$ & 0.0500 & 0.0540 & 0.0516 & 0.0015 \\
\hline \multicolumn{8}{|l|}{ Panel D: Decision Rate } \\
\hline FOMC Rate Decision (Upper Bound) & FR & 8 & $14: 15$ & 0.0250 & 0.0425 & 0.0338 & 0.0061 \\
\hline
\end{tabular}


TABLE 2.26: Pre-scheduled Macroeconomic Announcements Survey in Canada.

This table provides summary statistics for macroeconomic announcements according to 4 groups in the Canada in the year 2005.

\begin{tabular}{llllllll}
\hline Event & Source & Obs. & Time & Min & Max & Mean & Std. \\
\hline Panel A: Output & & & & & & & \\
Building Permits MoM & STCA & 12 & $08: 30$ & -0.0500 & 0.0400 & -0.0082 & 0.0270 \\
Housing Starts & STCA & 12 & $08: 30$ & $215 \mathrm{~K}$ & $230 \mathrm{~K}$ & $224.5 \mathrm{~K}$ & $4.48 \mathrm{~K}$ \\
Int'l Merchandise Trade & STCA & 12 & $08: 30$ & $4.3 \mathrm{~B}$ & $6.9 \mathrm{~B}$ & $5.19 \mathrm{~B}$ & $0.787 \mathrm{~B}$ \\
Manufacturing Sales MoM & STCA & 12 & $08: 30$ & -0.0060 & 0.0100 & 0.0038 & 0.0046 \\
Quarterly GDP Annualised & STCA & 4 & $08: 30$ & 0.0170 & 0.0360 & 0.0263 & 0.0078 \\
Retail Sales MoM & STCA & 12 & $08: 30$ & -0.0050 & 0.0140 & 0.0031 & 0.0065 \\
Wholesale Trade Sales MoM & STCA & 12 & $08: 30$ & 0.0020 & 0.0070 & 0.0050 & 0.0015 \\
\hline Panel B: Prices & & & & & & & \\
CPI YoY & STCA & 12 & $07: 00$ & 0.0180 & 0.0330 & 0.0233 & 0.0046 \\
Capacity Utilization Rate & STCA & 4 & $08: 30$ & 0.8560 & 0.8720 & 0.8625 & 0.0072 \\
Current Account Balance & STCA & 4 & $08: 30$ & $3.4 \mathrm{~B}$ & $9 \mathrm{~B}$ & $6.14 \mathrm{~B}$ & $2.51 \mathrm{~B}$ \\
Industrial Product Price MoM & STCA & 12 & $08: 30$ & -0.0100 & 0.0090 & 0.0030 & 0.0047 \\
New Housing Price Index MoM & STCA & 12 & $08: 30$ & 0.0030 & 0.0050 & 0.0039 & 0.0007 \\
Raw Materials Price Index MoM & STCA & 12 & $08: 30$ & -0.0200 & 0.0400 & 0.0148 & 0.0201 \\
\hline Panel C: Employment & & & & & & & \\
Labor Productivity QoQ & STCA & 4 & $08: 30$ & 0.0010 & 0.0050 & 0.0030 & 0.0016 \\
Unemployment Rate & STCA & 12 & $07: 00$ & 0.0660 & 0.0730 & 0.0687 & 0.0019 \\
\hline Panel D: Decision Rate & & & & & & & \\
Bank of Canada Rate Decision & BC & 8 & $09: 00$ & 0.0250 & 0.0325 & 0.0269 & 0.0029 \\
\hline
\end{tabular}

larly, I split pre-scheduled macroeconomic announcements in 4 Groups in the Canada. Table 2.26 presents the list of each group. I run the main regression model in equation (2.9) for the RV model for each economic group and explain the results in the following.

The first sample includes output announcements, which is presented in Panel A, Tables 2.25 and 2.26. Table 2.27 presents results of realised variance using output sample. The announcements of this sample come to the market at different times and overall there are 433 observations for the regression model. Spread at the best quote and Depth at the second 
TABLE 2.27: Realised Variance Using the Output Sample.

Results of regression of dependent variable $(R V)$ realised variance on liquidity measures: $S_{0}$ is spread, $D_{0}$ is the depth at the best quote, $D_{1}$ is the depth at the second best quote, AOF is the absolute order flow, AOI is the absolute order imbalance. Descriptions of the calculation of the variables can be found in Section 2.3. There are 433 observations in output announcements sample. The superscripts ***, **, and *indicate significance at the $1 \%, 5 \%$, and $10 \%$ level respectively. The significance is assessed using Newey-West standard errors.

\begin{tabular}{|c|c|c|c|c|c|}
\hline \multirow{2}{*}{ Variables } & \multicolumn{5}{|c|}{ Liquidity Measure (Output Announcements Sample) } \\
\hline & $\mathbf{S}_{0}$ & $\mathbf{D}_{0}$ & $\mathbf{D}_{1}$ & AOF & AOI \\
\hline Intercept & $\begin{array}{l}0.0824^{* * *} \\
(0.0236)\end{array}$ & $\begin{array}{l}0.0667^{* * *} \\
(0.0174)\end{array}$ & $\begin{array}{l}0.0604^{* * *} \\
(0.0150)\end{array}$ & $\begin{array}{l}0.0575^{* * *} \\
(0.0126)\end{array}$ & $\begin{array}{l}0.0632^{* * *} \\
(0.0126)\end{array}$ \\
\hline $\mathbf{1}_{\text {News }}$ & $\begin{array}{c}0.0234 \\
(0.0318)\end{array}$ & $\begin{array}{c}0.0394^{*} \\
(0.0227)\end{array}$ & $\begin{array}{c}0.0356 \\
(0.0231)\end{array}$ & $\begin{array}{c}0.0302^{*} \\
(0.0177)\end{array}$ & $\begin{array}{c}0.0380^{* *} \\
(0.0178)\end{array}$ \\
\hline $\mathbf{1}_{\text {Post }}$ & $\begin{array}{c}-0.0175 \\
(0.0352)\end{array}$ & $\begin{array}{c}-0.0134 \\
(0.0274)\end{array}$ & $\begin{array}{c}-0.0251 \\
(0.0274)\end{array}$ & $\begin{array}{c}-0.0002 \\
(0.0198)\end{array}$ & $\begin{array}{c}0.0009 \\
(0.0228)\end{array}$ \\
\hline $\mathbf{1}_{\text {News }} \mathbf{1}_{\text {Post }}$ & $\begin{array}{c}-0.0613 \\
(0.0719)\end{array}$ & $\begin{array}{c}0.0890^{*} \\
(0.0535)\end{array}$ & $\begin{array}{l}0.1425^{* *} \\
(0.0620)\end{array}$ & $\begin{array}{c}0.0783^{*} \\
(0.0467)\end{array}$ & $\begin{array}{c}0.0705 \\
(0.0478)\end{array}$ \\
\hline $\mathrm{RV}_{t-15}$ & $\begin{array}{l}0.3486^{* * *} \\
(0.1006)\end{array}$ & $\begin{array}{l}0.3481^{* * *} \\
(0.1031)\end{array}$ & $\begin{array}{l}0.3544^{* * *} \\
(0.1055)\end{array}$ & $\begin{array}{l}0.3245^{* * *} \\
(0.1006)\end{array}$ & $\begin{array}{l}0.3441^{* * *} \\
(0.1033)\end{array}$ \\
\hline $\mathrm{RV}_{t-15} \mathbf{1}_{\text {News }}$ & $\begin{array}{c}-0.0931 \\
(0.1455)\end{array}$ & $\begin{array}{c}-0.0894 \\
(0.1495)\end{array}$ & $\begin{array}{c}-0.1079 \\
(0.1460)\end{array}$ & $\begin{array}{c}-0.1090 \\
(0.1483)\end{array}$ & $\begin{array}{c}-0.0885 \\
(0.1469)\end{array}$ \\
\hline $\mathrm{RV}_{t-15} \mathbf{1}_{\text {Post }}$ & $\begin{array}{c}0.1409 \\
(0.2436)\end{array}$ & $\begin{array}{c}0.1339 \\
(0.2453)\end{array}$ & $\begin{array}{c}0.1606 \\
(0.2475)\end{array}$ & $\begin{array}{c}0.1316 \\
(0.2911)\end{array}$ & $\begin{array}{c}0.1403 \\
(0.2451)\end{array}$ \\
\hline $\mathrm{RV}_{t-15} \mathbf{1}_{\text {News }} \mathbf{1}_{\text {Post }}$ & $\begin{array}{c}-0.0988 \\
(0.3236)\end{array}$ & $\begin{array}{c}0.0598 \\
(0.3515)\end{array}$ & $\begin{array}{c}0.0396 \\
(0.3366)\end{array}$ & $\begin{array}{c}0.0567 \\
(0.3834)\end{array}$ & $\begin{array}{c}0.0557 \\
(0.3501)\end{array}$ \\
\hline $\mathrm{X}_{t}$ & $\begin{array}{c}-0.0592 \\
(0.0784)\end{array}$ & $\begin{array}{c}0.0004 \\
(0.0018)\end{array}$ & $\begin{array}{c}0.0009 \\
(0.0010)\end{array}$ & $\begin{array}{c}0.0005 \\
(0.0004)\end{array}$ & $\begin{array}{c}0.0021 \\
(0.0017)\end{array}$ \\
\hline $\mathrm{X}_{t} \mathbf{1}_{\text {News }}$ & $\begin{array}{c}0.0149 \\
(0.1003)\end{array}$ & $\begin{array}{c}-0.0024 \\
(0.0025)\end{array}$ & $\begin{array}{c}-0.0010 \\
(0.0019)\end{array}$ & $\begin{array}{c}-0.0002 \\
(0.0005)\end{array}$ & $\begin{array}{c}-0.0050^{*} \\
(0.0027)\end{array}$ \\
\hline $\mathrm{X}_{t} \mathbf{1}_{\text {Post }}$ & $\begin{array}{c}0.0396 \\
(0.1003)\end{array}$ & $\begin{array}{c}0.0009 \\
(0.0026)\end{array}$ & $\begin{array}{c}0.0018 \\
(0.0021)\end{array}$ & $\begin{array}{c}-0.0003 \\
(0.0006)\end{array}$ & $\begin{array}{c}-0.0032 \\
(0.0029)\end{array}$ \\
\hline $\mathrm{X}_{t} \mathbf{1}_{\text {News }} \mathbf{1}_{\text {Post }}$ & $\begin{array}{c}0.4007^{*} \\
(0.2408)\end{array}$ & $\begin{array}{c}-0.0018 \\
(0.0035)\end{array}$ & $\begin{array}{c}-0.0083^{* *} \\
(0.0039)\end{array}$ & $\begin{array}{c}-0.0002 \\
(0.0008)\end{array}$ & $\begin{array}{c}0.0019 \\
(0.0043)\end{array}$ \\
\hline $\operatorname{Sur}_{t} \mathbf{1}_{\text {Post }}$ & $\begin{array}{c}0.0279 \\
(0.0234)\end{array}$ & $\begin{array}{c}0.0211 \\
(0.0255)\end{array}$ & $\begin{array}{c}0.0172 \\
(0.0261)\end{array}$ & $\begin{array}{c}0.0273 \\
(0.0246)\end{array}$ & $\begin{array}{c}0.0220 \\
(0.0251)\end{array}$ \\
\hline Adjusted $R^{2}$ & 0.2416 & 0.1354 & 0.1450 & 0.1301 & 0.1347 \\
\hline
\end{tabular}


best quote as proxies for private information shock are significant at the $10 \%$ and $5 \%$ level respectively. Surprise as a proxy for public information shock is not significant. Hence, with the arrival of news in the output sample, spread at the best quote and depth at the second best quote are the main proxies that signal volatility increasing illiquidity in the market.

The second sample includes price announcements, which is presented in Panel B, Tables 2.25 and 2.26. Table 2.28 presents results of the regression model for this sample with 200 observations. depth at the best quote and absolute order flow as proxies of private information shocks are significant at the $10 \%$ and $1 \%$ level respectively. Surprise as a proxy for public information shock is not significant. Hence, with the arrival of macroeconomic announcements, the private information shocks cause illiquidity for the price sample and results in higher volatility.

The third sample includes employment announcements, which is presented in Panel C, Tables 2.25 and 2.26. Table 2.29 presents the results of regression model for this sample that includes 189 observations. For this sample, depth at the best quote and absolute order flow as proxies for private information shocks are significant at the $10 \%$ level. Surprise as the public information shock is significant for absolute order imbalance at the $10 \%$ level.

Finally, the last sample includes rate decision sample announcements, which is presented in Panel D, Tables 2.25 and 2.26. Table 2.30 reports the results for FOMC sample with 58 observations. Private information shocks are significant through depth at the second best quote, absolute order flow, and absolute order imbalance at the $1 \%, 5 \%$, and $5 \%$ level respectively. Public information shocks are not significant for the decision rate sample. Hence, when FOMC announcements come to the market, liquidity dries up through private information and causes higher volatility that can be captured through liquidity variables. 
TABLE 2.28: Realised Variance Using the Prices Sample.

Results of regression of dependent variable $(R V)$ realised variance on liquidity measures: $S_{0}$ is spread, $D_{0}$ is the depth at the best quote, $D_{1}$ is the depth at the second best quote, AOF is the absolute order flow, AOI is the absolute order imbalance. Descriptions of the calculation of the variables can be found in Section 2.3. There are 200 observations in Prices announcements sample. The superscripts ***,**, and *indicate significance at the 1\%,5\%, and $10 \%$ level respectively. The significance is assessed using Newey-West standard errors.

\begin{tabular}{|c|c|c|c|c|c|}
\hline \multirow{2}{*}{ Variables } & \multicolumn{5}{|c|}{ Liquidity Measure (Prices Announcements Sample) } \\
\hline & $\mathbf{S}_{0}$ & $\mathbf{D}_{0}$ & $\mathbf{D}_{1}$ & AOF & AOI \\
\hline Intercept & $\begin{array}{l}0.0722^{* * *} \\
(0.0212)\end{array}$ & $\begin{array}{l}0.0601^{* * *} \\
(0.0148)\end{array}$ & $\begin{array}{l}0.0562^{* * *} \\
(0.0124)\end{array}$ & $\begin{array}{l}0.0575^{* * *} \\
(0.0109)\end{array}$ & $\begin{array}{l}0.0580^{* * *} \\
(0.0113)\end{array}$ \\
\hline $\mathbf{1}_{\text {News }}$ & $\begin{array}{c}0.0467 \\
(0.0394)\end{array}$ & $\begin{array}{l}0.0743^{* * *} \\
(0.0281)\end{array}$ & $\begin{array}{l}0.0881^{* *} \\
(0.0361)\end{array}$ & $\begin{array}{l}0.0543^{* *} \\
(0.0232)\end{array}$ & $\begin{array}{l}0.0763^{* * *} \\
(0.0225)\end{array}$ \\
\hline $\mathbf{1}_{\text {Post }}$ & $\begin{array}{c}-0.0405 \\
(0.0268)\end{array}$ & $\begin{array}{c}0.0020 \\
(0.0216)\end{array}$ & $\begin{array}{c}-0.0080 \\
(0.0180)\end{array}$ & $\begin{array}{c}-0.0156 \\
(0.0154)\end{array}$ & $\begin{array}{c}0.0001 \\
(0.0177)\end{array}$ \\
\hline $\mathbf{1}_{\text {News }} \mathbf{1}_{\text {Post }}$ & $\begin{array}{c}0.0678 \\
(0.1113)\end{array}$ & $\begin{array}{l}0.2518^{* *} \\
(0.1302)\end{array}$ & $\begin{array}{c}0.2068 \\
(0.1560)\end{array}$ & $\begin{array}{c}0.2157^{*} \\
(0.1155)\end{array}$ & $\begin{array}{c}0.1802 \\
(0.1152)\end{array}$ \\
\hline $\mathrm{RV}_{t-15}$ & $\begin{array}{l}0.3605^{* * *} \\
(0.0480)\end{array}$ & $\begin{array}{l}0.3621^{* * *} \\
(0.0510)\end{array}$ & $\begin{array}{l}0.3606^{* * *} \\
(0.0527)\end{array}$ & $\begin{array}{l}0.3635^{* * *} \\
(0.0526)\end{array}$ & $\begin{array}{l}0.3644^{* * *} \\
(0.0511)\end{array}$ \\
\hline $\mathrm{RV}_{t-15} \mathbf{1}_{N \text { ews }}$ & $\begin{array}{r}-0.3336^{*} \\
(0.1883)\end{array}$ & $\begin{array}{r}-0.3513^{*} \\
(0.1925)\end{array}$ & $\begin{array}{r}-0.3696^{*} \\
(0.2054)\end{array}$ & $\begin{array}{c}-0.3997^{* *} \\
(0.1837)\end{array}$ & $\begin{array}{c}-0.3903^{* *} \\
(0.1871)\end{array}$ \\
\hline $\mathrm{RV}_{t-15} \mathbf{1}_{\text {Post }}$ & $\begin{array}{c}0.0136 \\
(0.1473)\end{array}$ & $\begin{array}{c}0.0132 \\
(0.1470)\end{array}$ & $\begin{array}{c}-0.0032 \\
(0.1443)\end{array}$ & $\begin{array}{c}-0.1631 \\
(0.1574)\end{array}$ & $\begin{array}{c}0.0001 \\
(0.1471)\end{array}$ \\
\hline $\mathrm{RV}_{t-15} \mathbf{1}_{\text {News }} \mathbf{1}_{\text {Post }}$ & $\begin{array}{c}0.6500 \\
(0.6002)\end{array}$ & $\begin{array}{l}1.1617^{* *} \\
(0.5841)\end{array}$ & $\begin{array}{c}0.6871 \\
(0.6846)\end{array}$ & $\begin{array}{l}1.1982^{* *} \\
(0.5631)\end{array}$ & $\begin{array}{c}1.0895^{*} \\
(0.5938)\end{array}$ \\
\hline $\mathrm{X}_{t}$ & $\begin{array}{c}-0.0512 \\
(0.0561)\end{array}$ & $\begin{array}{c}-0.0007 \\
(0.0016)\end{array}$ & $\begin{array}{c}0.0000 \\
(0.0009)\end{array}$ & $\begin{array}{c}-0.0001 \\
(0.0002)\end{array}$ & $\begin{array}{c}-0.0009 \\
(0.0018)\end{array}$ \\
\hline $\mathrm{X}_{t} \mathbf{1}_{\text {News }}$ & $\begin{array}{c}0.0464 \\
(0.0932)\end{array}$ & $\begin{array}{c}-0.0024 \\
(0.0031)\end{array}$ & $\begin{array}{c}-0.0032 \\
(0.0025)\end{array}$ & $\begin{array}{c}0.0004^{*} \\
(0.0002)\end{array}$ & $\begin{array}{c}-0.0056 \\
(0.0053)\end{array}$ \\
\hline $\mathrm{X}_{t} \mathbf{1}_{\text {Post }}$ & $\begin{array}{c}0.0923 \\
(0.0667)\end{array}$ & $\begin{array}{c}-0.0025 \\
(0.0025)\end{array}$ & $\begin{array}{c}-0.0001 \\
(0.0011)\end{array}$ & $\begin{array}{l}0.0010^{* *} \\
(0.0004)\end{array}$ & $\begin{array}{c}-0.0043 \\
(0.0028)\end{array}$ \\
\hline $\mathrm{X}_{t} \mathbf{1}_{\text {News }} \mathbf{1}_{\text {Post }}$ & $\begin{array}{c}0.2277 \\
(0.2357)\end{array}$ & $\begin{array}{r}-0.0211^{*} \\
(0.0120)\end{array}$ & $\begin{array}{c}-0.0014 \\
(0.0096)\end{array}$ & $\begin{array}{c}-0.0029^{* * *} \\
(0.0009)\end{array}$ & $\begin{array}{c}-0.0151 \\
(0.0176)\end{array}$ \\
\hline $\operatorname{Sur}_{t} \mathbf{1}_{\text {Post }}$ & $\begin{array}{c}-0.0017 \\
(0.0442)\end{array}$ & $\begin{array}{c}-0.0391 \\
(0.0586)\end{array}$ & $\begin{array}{r}-0.0315 \\
(0.0625)\end{array}$ & $\begin{array}{c}-0.0060 \\
(0.0560)\end{array}$ & $\begin{array}{c}-0.0329 \\
(0.0585)\end{array}$ \\
\hline Adjusted $\mathrm{R}^{2}$ & 0.3243 & 0.3104 & 0.2584 & 0.2907 & 0.2844 \\
\hline
\end{tabular}


TABLE 2.29: Realised Variance Using the Employment Sample.

Results of regression of dependent variable $(R V)$ realised variance on liquidity measures: $S_{0}$ is spread, $D_{0}$ is the depth at the best quote, $D_{1}$ is the depth at the second best quote, AOF is the absolute order flow, AOI is the absolute order imbalance. Descriptions of the calculation of the variables can be found in Section 2.3. There are 189 observations in employment announcements sample. The superscripts $* * *, * *$, and $*$ indicate significance at the $1 \%, 5 \%$, and $10 \%$ level respectively. The significance is assessed using Newey-West standard errors.

\begin{tabular}{|c|c|c|c|c|c|}
\hline \multirow{2}{*}{ Variables } & \multicolumn{5}{|c|}{ Liquidity Measure (Employment Announcements Sample) } \\
\hline & $\mathbf{S}_{0}$ & $\mathbf{D}_{0}$ & $\mathbf{D}_{1}$ & AOF & AOI \\
\hline Intercept & $\begin{array}{l}0.0722^{* * *} \\
(0.0212)\end{array}$ & $\begin{array}{l}0.0601^{* * *} \\
(0.0148)\end{array}$ & $\begin{array}{l}0.0562^{\text {*** }} \\
(0.0125)\end{array}$ & $\begin{array}{l}0.0575^{* * *} \\
(0.0109)\end{array}$ & $\begin{array}{l}0.0580^{* * *} \\
(0.0114)\end{array}$ \\
\hline $\mathbf{1}_{\text {News }}$ & $\begin{array}{c}-0.0139 \\
(0.0318)\end{array}$ & $\begin{array}{c}0.0468 \\
(0.0295)\end{array}$ & $\begin{array}{c}0.0170 \\
(0.0271)\end{array}$ & $\begin{array}{c}0.0298 \\
(0.0207)\end{array}$ & $\begin{array}{c}0.0404^{*} \\
(0.0243)\end{array}$ \\
\hline $\mathbf{1}_{\text {Post }}$ & $\begin{array}{c}-0.0405 \\
(0.0268)\end{array}$ & $\begin{array}{c}0.0020 \\
(0.0216)\end{array}$ & $\begin{array}{c}-0.0080 \\
(0.0180)\end{array}$ & $\begin{array}{c}-0.0156 \\
(0.0154)\end{array}$ & $\begin{array}{c}0.0001 \\
(0.0177)\end{array}$ \\
\hline $\mathbf{1}_{\text {News }} \mathbf{1}_{\text {Post }}$ & $\begin{array}{c}0.0992 \\
(0.0788)\end{array}$ & $\begin{array}{c}-0.0566 \\
(0.0945)\end{array}$ & $\begin{array}{c}-0.0029 \\
(0.1120)\end{array}$ & $\begin{array}{c}0.0746 \\
(0.1016)\end{array}$ & $\begin{array}{c}0.0341 \\
(0.1065)\end{array}$ \\
\hline $\mathrm{RV}_{t-15}$ & $\begin{array}{l}0.3605^{* * *} \\
(0.0481)\end{array}$ & $\begin{array}{l}0.3621^{* * *} \\
(0.0511)\end{array}$ & $\begin{array}{l}0.3606^{* * *} \\
(0.0528)\end{array}$ & $\begin{array}{l}0.3635^{* * *} \\
(0.0527)\end{array}$ & $\begin{array}{l}0.3644^{* * *} \\
(0.0512)\end{array}$ \\
\hline $\mathrm{RV}_{t-15} \mathbf{1}_{\text {News }}$ & $\begin{array}{c}-0.0537 \\
(0.1860)\end{array}$ & $\begin{array}{c}-0.0884 \\
(0.1927)\end{array}$ & $\begin{array}{c}-0.0065 \\
(0.1944)\end{array}$ & $\begin{array}{c}-0.0993 \\
(0.2265)\end{array}$ & $\begin{array}{c}-0.0826 \\
(0.1945)\end{array}$ \\
\hline $\mathrm{RV}_{t-15} \mathbf{1}_{\text {Post }}$ & $\begin{array}{c}0.0136 \\
(0.1476)\end{array}$ & $\begin{array}{c}0.0132 \\
(0.1472)\end{array}$ & $\begin{array}{c}-0.0032 \\
(0.1446)\end{array}$ & $\begin{array}{c}-0.1631 \\
(0.1577)\end{array}$ & $\begin{array}{c}0.0001 \\
(0.1473)\end{array}$ \\
\hline $\mathrm{RV}_{t-15} \mathbf{1}_{\text {News }} \mathbf{1}_{\text {Post }}$ & $\begin{array}{c}-0.1195 \\
(0.5201)\end{array}$ & $\begin{array}{c}-0.0194 \\
(0.5512)\end{array}$ & $\begin{array}{c}-0.0949 \\
(0.6501)\end{array}$ & $\begin{array}{c}0.3320 \\
(0.6052)\end{array}$ & $\begin{array}{c}0.1169 \\
(0.5525)\end{array}$ \\
\hline$X_{t}$ & $\begin{array}{c}-0.0512 \\
(0.0562)\end{array}$ & $\begin{array}{c}-0.0007 \\
(0.0016)\end{array}$ & $\begin{array}{c}0.0000 \\
(0.0009)\end{array}$ & $\begin{array}{c}-0.0001 \\
(0.0002)\end{array}$ & $\begin{array}{c}-0.0009 \\
(0.0018)\end{array}$ \\
\hline $\mathrm{X}_{t} \mathbf{1}_{\text {News }}$ & $\begin{array}{c}0.1462^{* *} \\
(0.0741)\end{array}$ & $\begin{array}{c}-0.0019 \\
(0.0030)\end{array}$ & $\begin{array}{c}0.0017 \\
(0.0024)\end{array}$ & $\begin{array}{c}0.0002 \\
(0.0003)\end{array}$ & $\begin{array}{c}-0.0018 \\
(0.0043)\end{array}$ \\
\hline $\mathrm{X}_{t} \mathbf{1}_{\text {Post }}$ & $\begin{array}{c}0.0923 \\
(0.0668)\end{array}$ & $\begin{array}{c}-0.0025 \\
(0.0025)\end{array}$ & $\begin{array}{c}-0.0001 \\
(0.0011)\end{array}$ & $\begin{array}{c}0.0010^{*} \\
(0.0004)\end{array}$ & $\begin{array}{c}-0.0043 \\
(0.0028)\end{array}$ \\
\hline $\mathrm{X}_{t} \mathbf{1}_{\text {News }} \mathbf{1}_{\text {Post }}$ & $\begin{array}{c}0.0364 \\
(0.0918)\end{array}$ & $\begin{array}{c}0.0299^{*} \\
(0.0171)\end{array}$ & $\begin{array}{c}0.0142 \\
(0.0190)\end{array}$ & $\begin{array}{c}-0.0011^{*} \\
(0.0007)\end{array}$ & $\begin{array}{c}0.0188 \\
(0.0171)\end{array}$ \\
\hline $\operatorname{Sur}_{t} \mathbf{1}_{\text {Post }}$ & $\begin{array}{c}0.0128 \\
(0.0290)\end{array}$ & $\begin{array}{c}0.0617 \\
(0.0432)\end{array}$ & $\begin{array}{c}0.0758 \\
(0.0482)\end{array}$ & $\begin{array}{c}0.0734 \\
(0.0502)\end{array}$ & $\begin{array}{c}0.0684^{*} \\
(0.0425)\end{array}$ \\
\hline Adjusted $\mathrm{R}^{2}$ & 0.4646 & 0.3131 & 0.2893 & 0.2561 & 0.2646 \\
\hline
\end{tabular}


TABLE 2.30: Realised Variance Using the Rate Decision Sample.

Results of regression of dependent variable $(R V)$ realised variance on liquidity measures: $S_{0}$ is spread, $D_{0}$ is the depth at the best quote, $D_{1}$ is the depth at the second best quote, AOF is the absolute order flow, AOI is the absolute order imbalance. Descriptions of the calculation of the variables can be found in Section 2.3. There are 58 observations in rate decision announcements sample. The superscripts ***,**, and *indicate significance at the $1 \%, 5 \%$, and $10 \%$ level respectively. The significance is assessed using Newey-West standard errors.

\begin{tabular}{|c|c|c|c|c|c|}
\hline \multirow{2}{*}{ Variables } & \multicolumn{5}{|c|}{ Liquidity Measure (Rate Decision Announcements Sample) } \\
\hline & $\mathbf{S}_{0}$ & $\mathbf{D}_{0}$ & $\mathbf{D}_{1}$ & AOF & AOI \\
\hline Intercept & $\begin{array}{c}-0.0013^{* * *} \\
(0.0214)\end{array}$ & $\begin{array}{l}0.0261^{* * *} \\
(0.0096)\end{array}$ & $\begin{array}{c}-0.0119^{* * *} \\
(0.0179)\end{array}$ & $\begin{array}{l}0.0306^{* * *} \\
(0.0096)\end{array}$ & $\begin{array}{l}0.0316^{* * *} \\
(0.0096)\end{array}$ \\
\hline $\mathbf{1}_{\text {News }}$ & $\begin{array}{c}0.0589 \\
(0.0488)\end{array}$ & $\begin{array}{c}0.0234 \\
(0.0390)\end{array}$ & $\begin{array}{l}0.1291^{* * *} \\
(0.0483)\end{array}$ & $\begin{array}{c}0.0243 \\
(0.0450)\end{array}$ & $\begin{array}{c}0.0347 \\
(0.0364)\end{array}$ \\
\hline $\mathbf{1}_{\text {Post }}$ & $\begin{array}{c}-0.0008 \\
(0.0247)\end{array}$ & $\begin{array}{c}-0.0407^{* * *} \\
(0.0142)\end{array}$ & $\begin{array}{c}-0.0060 \\
(0.0221)\end{array}$ & $\begin{array}{c}-0.0429^{* * *} \\
(0.0146)\end{array}$ & $\begin{array}{c}-0.0463^{* * *} \\
(0.0137)\end{array}$ \\
\hline $\mathbf{1}_{\text {News }} \mathbf{1}_{\text {Post }}$ & $\begin{array}{c}0.5323 \\
(0.1355)\end{array}$ & $\begin{array}{c}0.3491^{*} \\
(0.1826)\end{array}$ & $\begin{array}{c}0.2336 \\
(0.1808)\end{array}$ & $\begin{array}{c}0.0648 \\
(0.1922)\end{array}$ & $\begin{array}{l}0.3611^{* *} \\
(0.1536)\end{array}$ \\
\hline $\mathrm{RV}_{t-15}$ & $\begin{array}{c}0.3157 \\
(0.2588)\end{array}$ & $\begin{array}{c}0.2596 \\
(0.2531)\end{array}$ & $\begin{array}{c}0.1875 \\
(0.1406)\end{array}$ & $\begin{array}{c}0.3292 \\
(0.2515)\end{array}$ & $\begin{array}{c}0.2425 \\
(0.2518)\end{array}$ \\
\hline $\mathrm{RV}_{t-15} \mathbf{1}_{\text {News }}$ & $\begin{array}{c}-0.2967 \\
(0.5792)\end{array}$ & $\begin{array}{c}-1.1330 \\
(1.3446)\end{array}$ & $\begin{array}{c}-0.3398 \\
(0.4533)\end{array}$ & $\begin{array}{c}-0.3294 \\
(0.7130)\end{array}$ & $\begin{array}{c}-0.5327 \\
(0.5899)\end{array}$ \\
\hline $\mathrm{RV}_{t-15} \mathbf{1}_{\text {Post }}$ & $\begin{array}{c}0.9124 \\
(0.3629)\end{array}$ & $\begin{array}{l}0.9435^{* * *} \\
(0.3724)\end{array}$ & $\begin{array}{l}1.0246^{* * *} \\
(0.3085)\end{array}$ & $\begin{array}{l}0.8869^{* *} \\
(0.3702)\end{array}$ & $\begin{array}{l}0.9605^{* * *} \\
(0.3716)\end{array}$ \\
\hline $\mathrm{RV}_{t-15} \mathbf{1}_{\text {News }} \mathbf{1}_{\text {Post }}$ & $\begin{array}{c}1.1625 \\
(2.4138)\end{array}$ & $\begin{array}{c}-0.3855 \\
(1.9571)\end{array}$ & $\begin{array}{c}-1.0737 \\
(1.4010)\end{array}$ & $\begin{array}{c}0.3206 \\
(1.4569)\end{array}$ & $\begin{array}{c}-1.0814 \\
(1.5017)\end{array}$ \\
\hline $\mathrm{X}_{t}$ & $\begin{array}{c}0.0852 \\
(0.0583)\end{array}$ & $\begin{array}{c}-0.0004 \\
(0.0028)\end{array}$ & $\begin{array}{c}0.0069^{*} \\
(0.0035)\end{array}$ & $\begin{array}{c}-0.0005 \\
(0.0005)\end{array}$ & $\begin{array}{c}-0.0042 \\
(0.0036)\end{array}$ \\
\hline $\mathrm{X}_{t} \mathbf{1}_{\text {News }}$ & $\begin{array}{c}0.0959 \\
(0.0000)\end{array}$ & $\begin{array}{c}0.0203 \\
(0.0179)\end{array}$ & $\begin{array}{c}-0.0096^{* *} \\
(0.0041)\end{array}$ & $\begin{array}{c}0.0021 \\
(0.0022)\end{array}$ & $\begin{array}{l}0.0570^{* * *} \\
(0.0209)\end{array}$ \\
\hline $\mathrm{X}_{t} \mathbf{1}_{\text {Post }}$ & $\begin{array}{c}-0.1335 \\
(0.0677)\end{array}$ & $\begin{array}{c}0.0005 \\
(0.0029)\end{array}$ & $\begin{array}{c}-0.0065^{*} \\
(0.0036)\end{array}$ & $\begin{array}{c}0.0004 \\
(0.0006)\end{array}$ & $\begin{array}{c}0.0042 \\
(0.0037)\end{array}$ \\
\hline $\mathrm{X}_{t} \mathbf{1}_{\text {News }} \mathbf{1}_{\text {Post }}$ & $\begin{array}{c}-0.6887 \\
(0.4131)\end{array}$ & $\begin{array}{c}-0.0150 \\
(0.0185)\end{array}$ & $\begin{array}{l}0.0124^{* * *} \\
(0.0048)\end{array}$ & $\begin{array}{l}0.0112^{* *} \\
(0.0057)\end{array}$ & $\begin{array}{c}-0.0503^{* *} \\
(0.0217)\end{array}$ \\
\hline $\operatorname{Sur}_{t} \mathbf{1}_{\text {Post }}$ & $\begin{array}{c}0.0001 \\
(0.0000)\end{array}$ & $\begin{array}{c}0.0001 \\
(0.0000)\end{array}$ & $\begin{array}{c}0.0001 \\
(0.0000)\end{array}$ & $\begin{array}{c}0.0001 \\
(0.0000)\end{array}$ & $\begin{array}{c}0.0001 \\
(0.0000)\end{array}$ \\
\hline Adjusted $\mathrm{R}^{2}$ & 0.8060 & 0.7527 & 0.7902 & 0.8373 & 0.7607 \\
\hline
\end{tabular}




\subsection{Summary and Conclusion}

In this section, I present a summary and conclusion of all regression analysis. First, I demonstrate the significant results in a summary table and how they change with different subsamples. Then, I express an overall conclusion of my study.

\subsubsection{Summary of All Regression Analysis}

The main assumption of my study is that with the arrival of macroeconomic announcements the level of asymmetric information increases either through public information shocks or private information shocks. The liquidity in the market dries up due to uncertainty and different levels of information among traders, which results in a volatile market. Anyone with more information is prone to make profit whereas traders with less information are exposed to lose money. I set up regression model (2.9) (in Section 2.3.2) to analyse the impact of public and private information shocks on realised variance. Surprise is a proxy for public information shocks and a range of liquidity variables (Spread at the best quote $\left(S_{0}\right)$, depth at the best quote $\left(D_{0}\right)$, depth at the second best quote $\left(D_{1}\right)$, absolute order flow $(A O F)$, and absolute order imbalance $(A O I))$ are a proxy for private information shocks.

Table 2.31 presents the summary of significant results of all regressions. The main regression examines the impact of explanatory variables on realised variance through the Full, Relatively Important, and Most Important samples. Spread is the only significant variable in the Full sample at the $5 \%$ level and other samples do not reveal any significant results. The reason might be due to relatively or highly discussed information among traders. In my robustness tests, I remove the lag of Realised Variance from the regression model and results hold the same. When I remove the sur- 
prise variable from the regression model the significance level of spread increases from the $5 \%$ to $1 \%$ level. However, having the impact of surprise variable with positive or negative values does not change the primary results in the Full sample.

I evaluate the impact of macroeconomic announcements in the US and Canadian market separately and results hold the same for the US but no significant results are found for the Canadian announcements. Absolute return and modified absolute return are two alternative measures of volatility in the market. Using absolute return in the Full sample shows that spread at the best quote and absolute order flow are significant variables at the $10 \%$ and $5 \%$ level respectively. Absolute return in the Relatively Important sample shows that spread at the best quote is significant at the $10 \%$ level. Using modified absolute return in the Full sample shows that depth at the second best quote and absolute order flow are significant at the $1 \%$ and $5 \%$ level. Modified absolute return in the relatively important sample shows that depth at the second best quote, and absolute order flow are significant at the $1 \%$ and $10 \%$ level. The Most Important sample does not reveal any significant results for any sample, which might be due to these announcements being highly discussed among traders. The private information signal reveals through spread when I use absolute return as an alternative measure of volatility whereas it appears through depth at the second best quote and absolute order flow when I use modified absolute return.

Testing the impact of all variables jointly shows that spread at the best quote and depth at the second best quote are significant at the $10 \%$ level. Comparing all the results shows that the private information shocks is the main source of volatility compared with public information shocks. The sign of private information shocks reveals through different liquidity variables in different tests and we can not present a particular candidate that holds the same throughout all regression analysis. Having said 
TABLE 2.31: Summary of Significant Results.

This table reports the summary of significant results for main regression model and robustness tests. Liquidity variables as a sign of private information signal through different variables whereas surprise as a public information shock is not significant. Results of ${ }^{* * *},{ }^{* *}$, and * indicate significance at the $1 \%, 5 \%$, and $10 \%$ level respectively.

\begin{tabular}{|c|c|c|c|c|c|c|}
\hline Test & $\mathbf{S}_{0}$ & $\mathbf{D}_{0}$ & $\mathbf{D}_{1}$ & AOF & AOI & Sur \\
\hline \multicolumn{7}{|c|}{ Panel A: Significant Results in Using Full, Relative, and the Most Important Sample } \\
\hline \multicolumn{7}{|c|}{ A1: The Full Sample } \\
\hline $\mathrm{RV}$ & $* *$ & - & - & - & - & - \\
\hline $\mathrm{RV}_{N o-L a g}$ & $* *$ & - & - & - & - & - \\
\hline $\mathrm{RV}_{N o-S u r}$ & $* * *$ & - & - & - & - & - \\
\hline $\mathrm{RV}_{\text {Sur }}^{\text {Pos-Neg }}$ & $* *$ & - & - & - & - & - \\
\hline $\mathrm{RV}^{U S}$ & $* *$ & - & - & - & - & - \\
\hline $\mathrm{AR}$ & * & - & - & ** & - & - \\
\hline $\mathrm{R}^{r e s i}$ & - & - & $* * *$ & ** & - & - \\
\hline $\mathrm{RV}^{\text {Joint Variables }}$ & $* * *$ & - & - & - & - & - \\
\hline $\mathrm{RV}^{\text {All Times }}$ & $* * *$ & $* *$ & - & - & $* *$ & - \\
\hline \multicolumn{7}{|c|}{ A2: The Relatively Important Sample } \\
\hline $\mathrm{AR}$ & * & - & - & - & - & - \\
\hline $\mathrm{R}^{r e s i}$ & - & - & $* * *$ & * & - & - \\
\hline $\mathrm{RV}^{\text {All Times }}$ & * & - & $* *$ & - & $* *$ & - \\
\hline \multicolumn{7}{|c|}{ A3: The Most Important Sample } \\
\hline $\mathrm{RV}^{\text {Joint Variables }}$ & * & - & $*$ & - & - & - \\
\hline $\mathrm{RV}^{\text {All Times }}$ & - & * & - & - & - & - \\
\hline \multicolumn{7}{|c|}{ Panel B: Significant Results in Using Economic Samples } \\
\hline $\mathrm{RV}^{\text {Output }}$ & * & - & $* *$ & - & - & - \\
\hline $\mathrm{RV}^{\text {Prices }}$ & - & * & - & - & $* * *$ & - \\
\hline $\mathrm{RV}^{\text {Employment }}$ & - & * & - & $*$ & - & - \\
\hline $\mathrm{RV}^{\text {Decision Rate }}$ & - & - & $* * *$ & ** & $* *$ & - \\
\hline
\end{tabular}


that, spread is more likely to be the candidate compared with other liquidity variables. In robustness tests, I classify the macroeconomic announcements according to their economic functions: output, prices, employment, and decision rate. Results show that private information shocks are the main driver of volatility compared with public information shocks.

\subsubsection{Conclusion}

In this chapter, I use the limit order book to evaluate the impact of prescheduled macroeconomic announcements on realised variance in the Canadian FX market. I assert that the limit order book is one of the main sources of information: specifically asymmetric information and private information. I model the impact of information shocks through two main sources: public information shocks and private information shocks.

I find that public information shocks do not play much of a role in the Canadian FX market. On the other hand, private information is a key factor that explains volatility changes in the market. In other words, having access to private information (directly or indirectly) is the main driving force of shocks in the market, and has a significant effect on volatility. I exploit liquidity measures as a proxy for private information shocks. My results show that spread at the best quote is the most significant variable among all liquidity measures, and provides consistent results through my different samples and measures. Other liquidity measures do not reveal such robust results. I examine the macroeconomic announcements through the Full, Relatively Important, and Most Important samples. My results show that the Relatively Important and Most Important samples have less significant results than the Full sample, and posit that this could be due to extensive public analysis about more important news events, resulting in less scope for exploiting private information.

I extend the results through my robustness checks. The first robust- 
ness check considers absolute return and modified absolute return as two alternative proxies for realised variance. Although the results do not hold the same pattern for these different measures of volatility, they confirm my main result that private information shocks are more important than public information shocks. I give the caveat that, compared with the other two proxies, realised variance is able to take into account the impact of all quotes within the interval while the other proxies miss this effect. Hence, I ascribe the most importance to my realised variance results.

The second robustness check considers the effect of all liquidity measures jointly. This confirms that the effect of volatility changes comes through sensitivity to spread at the best quote, depth at the best quote, and absolute order imbalance liquidity measures. Finally, I examine the effect of news occurring at times other than at 8:30. My results confirm that private information shocks are the main driving force of volatility, and spread at the best quote is the main liquidity measure with significant and consistent results. I also evaluate the magnitude change of liquidity measures in smaller intervals of three 5-minute intervals before and after 8:30, between news days and no-news days. I analyse if they are large enough to make this sensitivity change economically significant. My results show that spread at the best quote and absolute order flow are the main liquidity measures with significant results, confirming spread's importance. 


\section{Chapter 3}

Optimal Enterprise Use Switching Policy 


\subsection{Introduction}

Real options valuation is an important area of research that is used in evaluating projects and investment decisions under uncertainty. I use this technique to evaluate a switching problem with two products 1 and 2. As product net incomes change, agents are incentivised to switch from one type of product to another. My models evaluate the switch frequency of relatively fast switching processes compared with prolonged ones. I conjecture that the latter involves higher uncertainty and time cost that is not captured precisely in the literature.

In this chapter, I examine the application of real options analyses to switching problems. I evaluate a situation where agents can switch between two different products in response to product net income changes. The term real options was coined by Myers (1977) and it refers to available options in an investment. There are a wide range of possible options in a project, namely: the option to defer, abandon, expand, contract, and switch. Real options analysis is widely used by scholars to evaluate investment decisions. ${ }^{1}$

I contribute to the literature in several ways. One of the common assumptions of real options to evaluate industry entry and exit is irreversibility. ${ }^{2}$ In this chapter, I relax this assumption to analyse real options to switching problems with reversibility. Reversibility is a spectrum that on one side there is costless reversibility where no real option exists. On the other side, there is costly complete irreversibility. In my models, agents have the option to switch from one product to another and reverse back to the original product. However, switches are costly, which suppresses the probability of switching. As a result, I am interested to know

${ }^{1}$ See, for example, McDonald and Siegel (1985a), Brennan and Schwartz (1985), McDonald and Siegel (1985b), Majd and Pindyck (1987), Carr (2009), Milne and Whalley (2000), Novy-Marx (2007), Guthrie (2007).

${ }^{2}$ In this chapter, reversibility means being able to go back to the original product. 
what explicit/implicit costs might occur in the switching process. The literature typically introduces a cash cost that captures the transition cost from one state to another. However, it is not clear to what extent does the cash cost capture all switching costs? Pure cost models may be appropriate when agents can switch relatively fast (e.g. livestock farming where farmers switch from farming sheep to cows and vice versa). However, in some activities, this is not necessarily true as there is also a waiting period before the predetermined amount of enterprise becomes productive again. For example, in horticulture, farmers face not only a cash cost but also a prolonged period of no production while new crops (i.e. saplings) reach maturity. In this regard, I extend the existing literature (e.g. Majd and Pindyck (1987) and Milne and Whalley (2000)) by adding a time cost (implicit/opportunity cost) as well as a cash cost (explicit cost). In other words, I evaluate the interaction between uncertainty, reversibility, timing, and switching costs at the same time in order to find the optimal policy. The importance of this approach is amplified when the transition is time consuming. Suppose an agent decides to switch to a lucrative product that entails a long switching process. The risk is that by the time the agent completes the transition process, the product net income may no longer be as lucrative, or the direction of switching may change. In this case, the agent not only loses the existing cash flows due to prolonged switching process but also ends up with a poor outcome, financially. Similarly, the reversibility option is exposed with the same risk. In the case where agents decide to reverse the process, they wait for a long time to go back to the original product. Hence, my advanced model (the cash-time model) has this feature to capture the time cost of switching precisely.

Among studies about real options, Song, Zhao, and Swinton (2011) use a real options framework to evaluate farmers' decisions to switch between traditional annual crops and perennial energy crops. Their results show that stochastic returns and government polices affect the optimal switching policy. The government can reduce the cost of converting land 
from corn-soybean to switch-grass by paying subsidies to incentivise farmers to make a one way switch. However, the indirect impact of this policy provides reverse incentives for farmers to switch from switch-grass to corn-soybean due to the trivial cost of reversing the land back to switchgrass. Nevertheless, the direct impact of this policy outweighs the indirect impact. My model differs from their model by taking into consideration the time cost and by giving the agents the option to reverse their decisions during the switch. In this regard, I conjecture that my model outperforms their model by capturing the explicit cost (the cash cost) as well as the implicit cost (the time cost) and may provide a better explanation for why agents may not act when there is a switching option or why the triggers of switching may vary from conventional cost-benefit analyses.

As I mentioned earlier, a part of the real options literature has focused on irreversible investments and entry/exit strategies. For instance, Shah and Ando (2016) examine the choice between conserving or converting land in a case study in Indonesia. Farmers in this study have the option to conserve the land and earn carbon credits, or convert the land to palm oil and earn returns from it. They analyse the financial and ecological cost of temporary and permanent land conservation policy incentives, which leads to policy advice. Also, Cunha-e-sa and Franco (2016) evaluate an irreversible real options problem in land redevelopment. They evaluate the residential rent changes and its impact on rural areas to switch to residential. This study advises that boundaries such as development halt periods and minimum-lot-sizes can postpone land conversion and help to protect open spaces. An example of entry/exit problem in agriculture is the evaluation of wine grape vineyard investment and disinvestment in north-west Victoria, Australia, by Seyoum-Tegegn and C. Chan (2013). They evaluate the entry/exit triggers of this industry under price and yield uncertainty using a real options approach, which is ignored by conventional cost-benefit analysis. Exploiting real options techniques makes them able to explain why investors might be in hysteresis and inaction zones as an 
optimal response, which results in lower exit and higher entry triggers. High vineyard establishment costs make investors reluctant to exit easily and wait for a better future. Similarly, Cyr, Hanagriffb, and Kwongc (2010) examine the wine industry of Texas in the US. Their results indicate that wine prices should increase by 30 to 40 percent to give incentive for investment in vineyard industry. They capture the hysteresis in investment in a real options framework. Tauer (2006) sets up an entry/exit real options problem to evaluate the dairy market in New York. He measures the optimal entry and exit milk prices where agents decide to invest or disinvest in this industry.

A part of real options studies evaluate switches with regime switching. Bollen (1999) evaluates real options to expand project and incorporates the product life cycle. His results show that the conventional method undervalue the option to contract and overvalue the option to expand. This results become more important in high technology industries. Bensoussan, Hoe, Yan, and Yin (2017) provide a mathematical solution for an irreversible investment that is examined in a duopoly games between leader and followers. They achieve an optimal investment solution under an uncertain cash flow regime and present implications in regime switching. There are more studies in this strand that are different from our approach in terms of methodology and underlying assumptions.

The remainder of this chapter proceeds as follows: In Section 3.2, I describe my methodology for solving a partial differential equation for two assets in order to find the optimal enterprise value with different products. In this regard, I derive the PDE for two competing products and then explain how to solve this PDE using finite differences and the alternating direction implicit algorithm. Section 3.3 presents the baseline parameter values and a range of scenarios to analyse. I simulate underlying product net income and generate statistics for optimal behaviour. Sections 3.3.3 and 3.3.4 evaluate my numerical results by analysing the opti- 
mal exercise frontiers and then provides sensitivity analysis for the model parameters. Finally, in Section 3.4, I summarise my results and provide advice for policy makers, agents, and future research.

\subsection{Methodology}

In this section, I explain how to derive and solve a partial differential equation (PDE) for enterprises with two competing products 1 and 2 . The PDE solution enables us to examine what product net income changes will cause agents to switch enterprise use. In this regard, I derive the PDE and employ its solution to construct three models for the switching problem, presenting the solution process in Section 3.2.1.

In this study, I assume a complete market. The importance of risk aversion depends on whether the market is complete or not. In a complete market, all products are tradable and agents maximise the value of their assets and work with risk neutral probabilities. In contrast, in an incomplete market, valuation will require equilibrium to be solved for, which in turn will depend on the utility function (and other instruments of) the agents concerned. I consider an agent (e.g. a farmer) who has access to a predetermined amount of enterprise (e.g. a parcel of land). At any point in time, this enterprise can be allocated to a variety of states, which will be discussed subsequently below. The enterprise value in all cases depends on the current value of the two potential products. The net income of two competing products, $S_{1}$ and $S_{2}$, are assumed to follow Geometric Brownian Motions (GBMs). I define the processes by the following stochastic differential equations:

$$
\begin{aligned}
& d S_{1}=\mu_{1} S_{1} d t+\sigma_{1} S_{1} d z_{1} \\
& d S_{2}=\mu_{2} S_{2} d t+\sigma_{2} S_{2} d z_{2}
\end{aligned}
$$


where $S_{i}$ is the product $i$ net income, $\mu_{i}$ and $\sigma_{i}$ are the drift and volatility of product $i, d t$ is a small interval of time, and $d z_{i}$ is an increment of a Brownian motion. The enterprise value is a function of product net income and time, $V_{i}\left(S_{1}, S_{2}, t\right)$. I use the Itô formula to derive the enterprise value process, as follows:

$$
\begin{aligned}
d V_{i} & =\frac{\partial V_{i}}{\partial S_{1}}\left[\mu_{1} S_{1} d t+\sigma_{1} S_{1} d z_{1}\right]+\frac{\partial V_{i}}{\partial S_{2}}\left[\mu_{2} S_{2} d t+\sigma_{2} S_{2} d z_{2}\right]+\frac{\partial V_{i}}{\partial t} d t \\
& +\frac{1}{2}\left[\frac{\partial^{2} V_{i}}{\partial S_{1}^{2}}\left[\sigma_{1}^{2} S_{1}^{2} d t\right]+\frac{\partial^{2} V_{i}}{\partial S_{2}^{2}}\left[\sigma_{2} S_{2}^{2} d t\right]+2 \frac{\partial^{2} V_{i}}{\partial S_{1} \partial S_{2}}\left[\rho \sigma_{1} \sigma_{2} S_{1} S_{2} d t\right]\right]
\end{aligned}
$$

Rearranging (3.2) and taking the expectation yields:

$$
\begin{aligned}
E\left(d V_{i}\right) & =\left[\mu_{1} S_{1} \frac{\partial V_{i}}{\partial S_{1}}+\mu_{2} S_{2} \frac{\partial V_{i}}{\partial S_{2}}+\frac{\partial V_{i}}{\partial t}+\frac{1}{2} \sigma_{1}^{2} S_{1}^{2} \frac{\partial^{2} V_{i}}{\partial S_{1}^{2}}+\frac{1}{2} \sigma_{2}^{2} S_{2}^{2} \frac{\partial^{2} V_{i}}{\partial S_{2}^{2}}\right. \\
& \left.+\rho \sigma_{1} \sigma_{2} S_{1} S_{2} \frac{\partial^{2} V_{i}}{\partial S_{1} \partial S_{2}}\right] d t,
\end{aligned}
$$

In equilibrium, the expected enterprise return must be equal to the capital gains over the time increment, $d t$ (i.e. $r V_{0} d t=E\left(d V_{0}\right)$ ). An active enterprise has an extra term (return) over time that should be added to the capital gain. The expected value obeys:

$$
r V_{i} d t=E\left(d V_{i}\right)+C F_{i}\left(S_{1}, S_{2}\right) d t,
$$

where $E\left(d V_{0}\right)$ is the expected change of enterprise value, $r$ is the (constant) fair expected return associated with enterprise ownership, $V_{0}$ is the inactive enterprise value that plays the role of transition state due to having the option to produce any of products, $d t$ is time increment, and $C F_{i}$ are cash flows in state $i$. Equating equations (3.3) and (3.4) and dividing by $d t$ 
gives the following PDE:

$$
\begin{aligned}
0 & =\frac{\partial V_{i}}{\partial t}+\mu_{1} S_{1} \frac{\partial V_{i}}{\partial S_{1}}+\mu_{2} S_{2} \frac{\partial V_{i}}{\partial S_{2}}+\frac{1}{2} \sigma_{1}^{2} S_{1}^{2} \frac{\partial^{2} V_{i}}{\partial S_{1}^{2}}+\frac{1}{2} \sigma_{2}^{2} S_{2}^{2} \frac{\partial^{2} V_{i}}{\partial S_{2}^{2}} \\
& +\rho \sigma_{1} S_{1} \sigma_{2} S_{2} \frac{\partial^{2} V_{i}}{\partial S_{1} \partial S_{2}}-r V_{i}+C F_{i} \\
& \equiv \frac{\partial V_{i}}{\partial t}+\mathcal{L} V_{i}+C F_{i}
\end{aligned}
$$

where $\mathcal{L}$ is the operator:

$$
\begin{aligned}
\mathcal{L} V & =\mu_{1} S_{1} \frac{\partial V}{\partial S_{1}}+\mu_{2} S_{2} \frac{\partial V}{\partial S_{2}}+\frac{1}{2} \sigma_{1}^{2} S_{1}^{2} \frac{\partial^{2} V}{\partial S_{1}^{2}}+\frac{1}{2} \sigma_{2}^{2} S_{2}^{2} \frac{\partial^{2} V}{\partial S_{2}^{2}} \\
& +\rho \sigma_{1} S_{1} \sigma_{2} S_{2} \frac{\partial^{2} V}{\partial S_{1} \partial S_{2}}-r V
\end{aligned}
$$

I solve the PDE (3.5) (see Section 3.2.1) with different state structures to evaluate agents' behaviour in a real options switching framework. I break my model into three models: (1) the cash-cost model, (2) the cashtime model, and (3) the projection model. The cash-cost model considers only the cash cost of switching, whereas the cash-time model not only considers the cash cost but also the time cost (opportunity cost). Finally, the projection model presents an approximation of the cash-time model, which is relatively faster in computation. To start with the cash-cost model, it is worth to know that the switching process is not always time consuming and in some cases such as livestock farming (e.g. beef and sheep) the process is faster compared with horticulture. Hence, the agents switch from one state to another by purely paying the cash cost (i.e. without going through a long waiting process). In this regard, I assume a scenario where agents have the option to have product 1 in state 1 and product 2 in state 2 . The enterprise value in each state comes through product net income. The enterprise optimisation equation for the cash-cost model has 
the following form:

$$
\begin{aligned}
& \mathcal{L} V_{i}+C F_{i} \leq 0 \\
& V_{i} \geq V_{j}-K_{i j, c c}
\end{aligned}
$$

where $\mathcal{L}$ is the operator (3.6), $V_{i}$ is the enterprise value in state $i, C F_{i}$ is the cash flow in state $i, V_{j}$ is the enterprise value in state $j$, and $K_{i j, c c}$ is the cash cost to be paid and switch from state $i$ to state $j$. However, in reality switches are not always instantaneous and there are switches (e.g. horticulture) that require a prolonged process. Note that the problem is elliptic since I do not have the $\left(\frac{\partial V_{i}}{\partial t}\right)$ term. We set up the cash-time model by assuming two productive states 1 and 2, and a transition state in between that agents cross to transfer from product 1 to 2 and vice versa. The productive states have the following options:

$$
\begin{aligned}
& \mathcal{L} V_{i}+C F_{i} \leq 0 \\
& V_{i} \geq V_{i, u}(0)-K_{i, u},
\end{aligned}
$$

where $V_{i, u}(0)$ is the enterprise value where removing product $i$ has just started, and $K_{i, u}$ is the upfront cash cost of removing product $i$. Note that the problem is elliptic since I do not have the $\left(\frac{\partial V_{i}}{\partial t}\right)$ term. Agents have incentives to switch between products based on their respective net income. Suppose agents who are producing product 1 realise that the competing product (2) is more lucrative. Hence, they have an incentive to switch from product 1 to product 2 . However, in the interim, this results in having an enterprise with no net income, called the "transition" state. The transition state provides the value of removing. The removing process takes time, which could be relatively fast and inexpensive for some products, but could be lengthy and expensive if preparation is required. We model 
the transition state as follows:

$$
\begin{aligned}
& V_{i, u}\left(T_{i}^{\text {Remove }}\right)=V_{T r} \\
& \mathcal{L} V_{i, u}+\frac{\partial V_{i, u}}{\partial t}-k_{i, u}=0
\end{aligned}
$$

where $V_{i, u}(t)$ is the enterprise value $t$ years into the removing process, $T_{i}^{\text {Remove }}$ is the time taken to remove product $i, V_{T r}$ is the transition enterprise value, the term $\frac{\partial V_{i, u}}{\partial t}$ accounts for the fact that time now affects enterprise value and I solve a parabolic PDE, and $k_{i, u}$ is the ongoing cost of removing product $i$ that is incurred throughout the removing process. When agents finish the removing process, they are in the transition state, whose value obeys:

$$
\begin{aligned}
& \mathcal{L} V_{T r} \leq 0 \\
& V_{T r} \geq \max \left(V_{1, p}(0)-K_{1, p}, V_{2, p}(0)-K_{2, p}\right)
\end{aligned}
$$

where $V_{i, p}(0)$ is the enterprise value where product $i$ has just been initiated (but has not yet reached productivity), and $K_{i, p}$ is the upfront cash cost of initialising product $i$. Agents in the transition state have the option to continue the switching process and initialise another product (or go back to the old product). However, the initialisation process takes time and is potentially slow and expensive. The enterprise value where initialisation is taking place solves:

$$
\begin{aligned}
& V_{i, p}\left(T_{i}^{\text {Initiate }}\right)=V_{i} \\
& \mathcal{L} V_{i, p}+\frac{\partial V_{i, p}}{\partial t}-k_{i, p} \leq 0 \\
& V_{i, p} \geq V_{i, u}(0)-K_{i, u},
\end{aligned}
$$

where $T_{i}^{\text {Initiate }}$ is the time taken to initialise product $i$, and $k_{i, p}$ is the on- 
going cash cost throughout the initialisation process. Note that, like the removal state, here I solve a parabolic PDE.

Finally, the cash-time model can be approximated by the projection model, which computes the time cost without capturing the removal and initialisation process formally. Agents in the projection model switch from one product to another or go back to the original product instantaneously. All the waiting cost (in terms of the forgone profits) is modeled as the extra monetary cost of switching to be paid by agents. The projection model approximates the time cost as follows:

$$
K_{i j, t c}=\int_{0}^{T_{i, u}} k_{i, u} e^{-r t} d t+\int_{T_{i, u}}^{T_{i, u}+T_{j, p}} k_{j, p} e^{-r t} d t+\frac{S_{i}}{\mu_{i}-r}\left[e^{\left(\mu_{i}-r\right)\left(T_{i, u}+T_{j, p}\right)}-1\right],
$$

where $K_{i j, t c}$ is the estimated time cost of switching from product $i$ to $j, T_{i, u}$ is the time to remove product $i, T_{j, p}$ is the time to initialise product $j$, and $\mu_{i}, r$, and $S_{i}$ are drift, discount rate, and cash flow of product $i$ respectively. The enterprise value in the projection model follows the following form:

$$
\begin{aligned}
& \mathcal{L} V_{i} \leq 0 \\
& V_{i} \geq V_{j}-K_{i j, t c}-K_{i j, c c},
\end{aligned}
$$

where $K_{i j, c c}$ is the upfront cash cost of removing product $i$ and initialising product $j$ (i.e. $K_{i, u}+K_{j, p}$ ). We conjecture that the frequency of switching increases in the projection model compared with the cash-time model due to the faster switching process. To this end, the cash-cost model neither computes the time cost formally nor approximates it, and focuses on only the cash cost. In contrast, the cash-time model by considering the exact time cost and the projection model by approximating the time cost capture a higher cost of switching. Hence, I conjecture that the frequency of switching in the cash-cost model will be considerably higher than the latter models since it overlooks the time cost entirely. 


\subsubsection{Solution Method}

In this part, I present my solution technique for the PDE (3.5). The implicit Finite Difference Method (FDM) provides a stable solution. However, when the system of equations has a dimension greater than one, I lose the computational advantage of having a tridiagonal matrix. The solution to overcome this difficulty and make my computation more efficient is to use the Alternating Direction Implicit (ADI) method, which is a numerical approach to solving PDEs. Before applying the ADI method, I transform product net income into natural logarithms $x_{1}=\ln \left(S_{1}\right), x_{2}=\ln \left(S_{2}\right)$ and derive the corresponding partial derivatives as follows:

$$
\begin{aligned}
\frac{\partial V_{i}}{\partial S_{1}} & =\frac{\partial V_{i}}{\partial x_{1}} \frac{\partial x_{1}}{\partial S_{1}}=\frac{\partial V_{i}}{\partial x_{1}} \frac{1}{S_{1}} \\
\frac{\partial V_{i}}{\partial S_{2}} & =\frac{\partial V_{i}}{\partial x_{2}} \frac{\partial x_{2}}{\partial S_{2}}=\frac{\partial V_{i}}{\partial x_{2}} \frac{1}{S_{2}} \\
\frac{\partial^{2} V_{i}}{\partial S_{1}^{2}} & =\frac{\partial}{\partial S_{1}} \frac{\partial V_{i}}{\partial S_{1}}=\frac{\partial}{\partial S_{1}}\left(\frac{\partial V_{i}}{\partial x_{1}} \frac{1}{S_{1}}\right)=\frac{1}{S_{1}} \frac{\partial^{2} V_{i}}{\partial x_{1}^{2}} \frac{1}{S_{1}}+\frac{\partial V_{i}}{\partial x_{1}}\left(-\frac{1}{S_{1}^{2}}\right) \\
& =\frac{1}{S_{1}^{2}} \frac{\partial^{2} V_{i}}{\partial x_{1}^{2}}-\frac{1}{S_{1}^{2}} \frac{\partial V_{i}}{\partial x_{1}} \\
\frac{\partial^{2} V_{i}}{\partial S_{2}^{2}} & =\frac{\partial}{\partial S_{2}} \frac{\partial V_{i}}{\partial S_{2}}=\frac{\partial}{\partial S_{2}}\left(\frac{\partial V_{i}}{\partial x_{2}} \frac{1}{S_{2}}\right)=\frac{1}{S_{2}} \frac{\partial^{2} V_{i}}{\partial x_{2}^{2}} \frac{1}{S_{2}}+\frac{\partial V_{i}}{\partial x_{2}}\left(-\frac{1}{S_{2}^{2}}\right) \\
\frac{\partial^{2} V_{i}}{\partial S_{1} \partial S_{2}} & =\frac{1}{\partial S_{2}} \frac{\partial^{2} V_{i}}{\partial x_{2}^{2}}-\frac{1}{S_{2}^{2}} \frac{\partial V_{i}}{\partial x_{2}} \\
& =\frac{\partial}{\partial S_{2}}\left(\frac{\partial V_{i}}{\partial x_{1}} \frac{1}{S_{1}}\right)=\frac{1}{S_{1}} \frac{1}{S_{2}} \frac{\partial^{2} V_{i}}{\partial x_{1} \partial x_{2}},
\end{aligned}
$$


I substitute the derivatives from equation (3.14) into equation (3.5) and derive the following PDE.

$$
\frac{\partial V_{i}}{\partial t}=\nu_{1} \frac{\partial V_{i}}{\partial x_{1}}+\nu_{2} \frac{\partial V_{i}}{\partial x_{2}}+\frac{1}{2} \sigma_{1}^{2} \frac{\partial^{2} V_{i}}{\partial x_{1}^{2}}+\frac{1}{2} \sigma_{2}^{2} \frac{\partial^{2} V_{i}}{\partial x_{2}^{2}}+\rho \sigma_{1} \sigma_{2} \frac{\partial^{2} V_{i}}{\partial x_{1} \partial x_{2}}-r V_{i}+e^{x_{i}},
$$

where $\nu_{1}$ is $\left(\mu_{1}-\frac{1}{2} \sigma_{1}^{2}\right)$ and $\nu_{2}$ is $\left(\mu_{2}-\frac{1}{2} \sigma_{2}^{2}\right)$.

The ADI method is a powerful way of solving multidimensional PDEs. However, the ADI is not initially developed to handle cross-partial terms. Therefore, in order to solve equation (3.15), I need to use a transformation to remove the $\frac{\partial^{2} V_{i}}{\partial x_{1} \partial x_{2}}$ term. Clewlow and Strickland (1998) propose to compute the eigenvectors and eigenvalues of the covariance matrix of $x_{1}$ and $x_{2}$.

$$
\left[\begin{array}{cc}
\sigma_{1}^{2} & \rho \sigma_{1} \sigma_{2} \\
\rho \sigma_{1} \sigma_{2} & \sigma_{2}^{2}
\end{array}\right]=\left[\begin{array}{cc}
e_{11} & e_{21} \\
e_{12} & e_{22}
\end{array}\right]\left[\begin{array}{cc}
\lambda_{1} & 0 \\
0 & \lambda_{2}
\end{array}\right]\left[\begin{array}{cc}
e_{11} & e_{12} \\
e_{21} & e_{22}
\end{array}\right]
$$

where on the left hand side, I have covariance matrix of $x_{1}$ and $x_{2}$ and on the right hand side, the eigenvalue decomposition. The eigenvectors give the linear combinations of $x_{1}$ and $x_{2}$, which are uncorrelated:

$$
\begin{gathered}
{\left[\begin{array}{l}
y_{1} \\
y_{2}
\end{array}\right]=\left[\begin{array}{ll}
e_{11} & e_{12} \\
e_{21} & e_{22}
\end{array}\right]\left[\begin{array}{l}
x_{1} \\
x_{2}
\end{array}\right]=\left[\begin{array}{l}
e_{11} x_{1}+e_{12} x_{2} \\
e_{21} x_{1}+e_{22} x_{2}
\end{array}\right]} \\
{\left[\begin{array}{l}
x_{1} \\
x_{2}
\end{array}\right]=\left[\begin{array}{ll}
e_{11} & e_{12} \\
e_{21} & e_{22}
\end{array}\right]\left[\begin{array}{l}
y_{1} \\
y_{2}
\end{array}\right]=\left[\begin{array}{l}
e_{11} y_{1}+e_{12} y_{2} \\
e_{21} y_{1}+e_{22} y_{2}
\end{array}\right]} \\
{\left[\begin{array}{l}
\alpha_{1} \\
\alpha_{2}
\end{array}\right]=\left[\begin{array}{ll}
e_{11} & e_{12} \\
e_{21} & e_{22}
\end{array}\right]\left[\begin{array}{l}
v_{1} \\
v_{2}
\end{array}\right]=\left[\begin{array}{l}
e_{11} v_{1}+e_{12} v_{2} \\
e_{21} v_{1}+e_{22} v_{2}
\end{array}\right],}
\end{gathered}
$$

Now I use equation 3.16 to derive the corresponding derivatives of equa- 
tion 3.15 with respect to $x_{1}$ and $x_{2}$ as follows:

$$
\begin{aligned}
\frac{\partial V_{i}}{\partial x_{1}} & =\frac{\partial V_{i}}{\partial y_{1}} \cdot \frac{\partial y_{1}}{\partial x_{1}}+\frac{\partial V_{i}}{\partial y_{2}} \cdot \frac{\partial y_{2}}{\partial x_{1}}=\frac{\partial V_{i}}{\partial y_{1}} \cdot e_{11}+\frac{\partial V_{i}}{\partial y_{2}} \cdot e_{21} \\
\frac{\partial V_{i}}{\partial x_{2}} & =\frac{\partial V_{i}}{\partial y_{1}} \cdot \frac{\partial y_{1}}{\partial x_{2}}+\frac{\partial V_{i}}{\partial y_{2}} \cdot \frac{\partial y_{2}}{\partial x_{2}}=\frac{\partial V_{i}}{\partial y_{1}} \cdot e_{12}+\frac{\partial V_{i}}{\partial y_{2}} \cdot e_{22} \\
\frac{\partial^{2} V_{i}}{\partial x_{1}^{2}} & =e_{11}\left[\frac{\partial^{2} V_{i}}{\partial y_{1}^{2}} \cdot \frac{\partial y_{1}}{\partial x_{1}}+\frac{\partial^{2} V_{i}}{\partial y_{1} \partial y_{2}} \cdot \frac{\partial y_{2}}{\partial x_{1}}\right]+e_{21}\left[\frac{\partial^{2} V_{i}}{\partial y_{2}^{2}} \cdot \frac{\partial y_{2}}{\partial x_{1}}+\frac{\partial^{2} V_{i}}{\partial y_{1} \partial y_{2}} \cdot \frac{\partial y_{1}}{\partial x_{1}}\right] \\
& =e_{11}^{2} \cdot \frac{\partial^{2} V_{i}}{\partial y_{1}^{2}}+2 \cdot e_{11} \cdot e_{21} \cdot \frac{\partial^{2} V_{i}}{\partial y_{1} \partial y_{2}}+e_{21}^{2} \cdot \frac{\partial^{2} V_{i}}{\partial y_{2}^{2}} \\
\frac{\partial^{2} V_{i}}{\partial x_{2}^{2}} & =e_{12}\left[\frac{\partial^{2} V_{i}}{\partial y_{1}^{2}} \cdot \frac{\partial y_{1}}{\partial x_{2}}+\frac{\partial^{2} V_{i}}{\partial y_{1} \partial y_{2}} \cdot \frac{\partial y_{2}}{\partial x_{2}}\right]+e_{22}\left[\frac{\partial^{2} V_{i}}{\partial y_{2}^{2}} \cdot \frac{\partial y_{2}}{\partial x_{2}}+\frac{\partial^{2} V_{i}}{\partial y_{1} \partial y_{2}} \cdot \frac{\partial y_{1}}{\partial x_{2}}\right] \\
& =e_{12}^{2} \cdot \frac{\partial^{2} V_{i}}{\partial y_{1}^{2}}+2 \cdot e_{12} \cdot e_{22} \cdot \frac{\partial^{2} V_{i}}{\partial y_{1} \partial y_{2}}+e_{22}^{2} \cdot \frac{\partial^{2} V_{i}}{\partial y_{2}^{2}} \\
\frac{\partial^{2} V_{i}}{\partial x_{1} \partial x_{2}} & =e_{11}\left[\frac{\partial^{2} V_{i}}{\partial y_{1}^{2}} \cdot \frac{\partial y_{1}}{\partial x_{2}}+\frac{\partial V_{i}}{\partial y_{1} \partial y_{2}} \cdot \frac{\partial y_{2}}{\partial x_{2}}\right]+e_{21}\left[\frac{\partial^{2} V_{i}}{\partial y_{1} \partial y_{2}} \cdot \frac{\partial y_{1}}{\partial x_{2}}+\frac{\partial^{2} V_{i}}{\partial y_{2}^{2}} \cdot \frac{\partial y_{2}}{\partial x_{2}}\right] \\
& =e_{11} \cdot e_{12} \cdot \frac{\partial^{2} V_{i}}{\partial y_{1}^{2}}+e_{11} \cdot e_{22} \cdot \frac{\partial^{2} V_{i}}{\partial y_{1} \partial y_{2}}+e_{21} \cdot e_{12} \frac{\partial^{2} V_{i}}{\partial y_{1} \partial y_{2}}+e_{21} \cdot e_{22} \cdot \frac{\partial y_{2}^{2}}{(3.17)}
\end{aligned}
$$

Substituting the corresponding derivatives from (3.17) into (3.15) and simplifying the terms gives:

$$
\frac{\partial V_{i}}{\partial t}=\alpha_{1} \frac{\partial V_{i}}{\partial y_{1}}+\alpha_{2} \frac{\partial V_{i}}{\partial y_{2}}+\frac{1}{2} \lambda_{1} \frac{\partial^{2} V_{i}}{\partial y_{1}^{2}}+\frac{1}{2} \lambda_{2} \frac{\partial^{2} V_{i}}{\partial y_{2}^{2}}-r V_{i}+e^{\left(e_{i 1} y_{1}+e_{i 2} y_{2}\right)}
$$

Generally, in numerical analysis, I replace continuous PDEs with discrete approximations, which reduce continuous differential equations into equivalent linear equation systems. I discretise my partial differential equation according to the corresponding dimensions and approximate 
the solution at isolated points rather than developing the solution everywhere. Hence, I define $\underline{y_{i}}$ and $\overline{y_{i}}$ as the lower and upper bounds with $N_{i}$ number of steps in dimension $y_{i}$. In general, the greater the number of points, the more accurate the solution. According to the type of finite difference in approximation, there will be errors called truncation errors. I can construct finite difference approximations of different accuracy by defining a linear combination of function values and expanding the values by Taylor series. As an example, suppose I want to find the second order approximation for the first derivative with a forward finite difference using three steps, I have the following set up:

$$
\frac{\partial V}{\partial y_{1}}=\frac{a V\left(y_{1}, y_{2}\right)+b V\left(y_{1}+\Delta y_{1}, y_{2}\right)+c V\left(y_{1}+2 \Delta y_{1}, y_{2}\right)}{\Delta y_{1}}+O\left(\Delta y_{1}\right)^{2},
$$

where $O\left(\Delta y_{1}\right)^{2}$ is the truncation error or high order approximation that I neglect in my approximation. I need to find the coefficients $a, b$, and $c$. This is a straight forward process if I use the Taylor series to expand the values in the $y_{1}$ dimension as follows:

$$
\begin{gathered}
V\left(y_{1}+\Delta y_{1}\right)=V\left(y_{1}\right)+\Delta y_{1} \frac{\partial V}{\partial y_{1}}+\frac{\left(\Delta y_{1}\right)^{2}}{2 !} \frac{\partial^{2} V}{\partial y_{1}^{2}}+\frac{\left(\Delta y_{1}\right)^{3}}{3 !} \frac{\partial^{3} V}{\partial y_{1}^{3}}+O\left(\Delta y_{1}\right)^{4} \\
V\left(y_{1}+2 \Delta y_{1}\right)=V\left(y_{1}\right)+2 \Delta y_{1} \frac{\partial V}{\partial y_{1}}+4 \frac{\left(\Delta y_{1}\right)^{2}}{2 !} \frac{\partial^{2} V}{\partial y_{1}^{2}}+8 \frac{\left(\Delta y_{1}\right)^{3}}{3 !} \frac{\partial^{3} V}{\partial y_{1}^{3}}+O\left(\Delta y_{1}\right)^{4},
\end{gathered}
$$

I substitute the corresponding approximations of $y_{1}$ with forward steps into (3.19). As a result, the following system of equations should be solved:

$$
\left[\begin{array}{lll}
1 & 1 & 1 \\
0 & 1 & 2 \\
0 & 1 & 4
\end{array}\right]\left[\begin{array}{l}
a \\
b \\
c
\end{array}\right]=\left[\begin{array}{l}
0 \\
1 \\
0
\end{array}\right],
$$

Solving this system of equations gives $a=-1.5, b=2$, and $c=-0.5$. Hence, the second order approximation of the first derivative with three 
steps forward is as follows:

$$
\frac{\partial V}{\partial y_{1}}=\frac{-3 V\left(y_{1}, y_{2}\right)+4 V\left(y_{1}+\Delta y_{1}, y_{2}\right)-V\left(y_{1}+2 \Delta y_{1}, y_{2}\right)}{2 \Delta y_{1}}+O\left(\Delta y_{1}\right)^{2}
$$

Similarly, for all the other finite difference approximations, I construct the function based on the order of accuracy, order of derivative, and the scheme type (forward, backward, or central). To this end, I employ the Peaceman and Rachford (1955) ADI method to solve my PDE in equation (3.18) for the first and second derivatives with second order accuracy. I discretise the spatial terms of the operator as the discrete operator $\mathcal{L}_{y_{i}}^{\text {mid }}$ when considering a point on the interior of the grid (i.e. $\underline{y}_{i}<y_{i}<\overline{y_{i}}$ ):

$$
\begin{aligned}
\mathcal{L}_{y_{i}}^{m i d} V & =\alpha_{i}\left[\frac{V_{y_{i}+\Delta y_{i}, y_{j}}-V_{y_{i}-\Delta y_{i}, y_{j}}}{2 \Delta y_{i}}\right]+\frac{1}{2} \lambda_{i}\left[\frac{V_{y_{i}+\Delta y_{i}, y_{j}}-2 V_{y_{i}, y_{j}}+V_{y_{i}-\Delta y_{i}, y_{j}}}{\left(\Delta y_{i}\right)^{2}}\right] \\
& -r V_{y_{i}, y_{j}}
\end{aligned}
$$

where if $i=1$ then $j=2$ and vice versa. Similarly, I define operators $\mathcal{L}_{y_{i}}^{\mathcal{L B}}$ and $\mathcal{L}_{y_{i}}^{\mathcal{U B}}$ for the equations system as follows:

$$
\begin{aligned}
\mathcal{L}_{y_{i}}^{\mathcal{L B}} V & =\alpha_{i}\left[\frac{-3 V_{y_{i}, y_{j}}+4 V_{y_{i}+\Delta y_{i}, y_{j}}-V_{y_{i}+2 \Delta y_{i}, y_{j}}}{2 \Delta y_{i}}\right] \\
& +\frac{1}{2} \lambda_{i}\left[\frac{2 V_{y_{i}, y_{j}}-5 V_{y_{i}+\Delta y_{i}, y_{j}}+4 V_{y_{i}+2 \Delta y_{i}, y_{j}}-V_{y_{i}+3 \Delta y_{i}, y_{j}}}{\left(\Delta y_{i}\right)^{2}}\right]-r V_{y_{i}, y_{j}} \\
\mathcal{L}_{y_{i}}^{\mathcal{U B}} V & =\alpha_{i}\left[\frac{3 V_{y_{i}, y_{j}}-4 V_{y_{i}-\Delta y_{i}, y_{j}}+V_{y_{i}-2 \Delta y_{i}, y_{j}}}{2 \Delta y_{i}}\right] \\
& +\frac{1}{2} \lambda_{i}\left[\frac{2 V_{y_{i}, y_{j}}-5 V_{y_{i}-\Delta y_{i}, y_{j}}+4 V_{y_{i}-2 \Delta y_{i}, y_{j}}-V_{y_{i}-3 \Delta y_{i}, y_{j}}}{\left(\Delta y_{i}\right)^{2}}\right]-r V_{y_{i}, y_{j}}
\end{aligned}
$$

where if $i=1$ then $j=2$ and vice versa. The system of equations using the 
middle equations and boundaries is built up as follows:

$$
\mathcal{L}_{y_{i}}=\left\{\begin{array}{lll}
\mathcal{L}_{y_{i}}^{\text {mid }}, & \text { if } & \underline{y}_{i}<y_{i}<\overline{y_{i}} \\
\mathcal{L}_{y_{i}}^{\mathcal{L B}}, & \text { if } & y_{i}=\underline{y_{i}} \\
\mathcal{L}_{y_{i}}^{\mathcal{U B}}, & \text { if } & y_{i}=\overline{y_{i}}
\end{array}\right.
$$

To solve theses systems of equations, I use the ADI method, which is widely used to solve the PDEs in numerical analysis. This method was first used by Peaceman and Rachford (1955) and Douglas and Rachford (1955). The ADI method has the advantage of being unconditionally stable, as well as being second order accurate. I can also reduce the systems of equations that must be solved to being tridiagonal, resulting in fast computation of solutions. The ADI method works by solving the PDE using two partial time steps instead of a full time step. In the first partial time step, I solve the system of equation by having $y_{1}$ explicit and $y_{2}$ implicit (solving in rows), and then switch the direction by having $y_{1}$ implicit and $y_{2}$ explicit (solving in columns). I solve the following systems of equations at each time step:

$$
\begin{gathered}
0=\left[\frac{V_{y_{1}, y_{2}}^{t}-V_{y_{1}, y_{2}}^{t-\frac{\Delta t}{2}}}{\frac{\Delta t}{2}}\right]+\mathcal{L}_{y_{1}} V^{t}+\mathcal{L}_{y_{2}} V^{t-\frac{\Delta t}{2}}+C F\left(y_{1}, y_{2}\right) \\
0=\left[\frac{V_{y_{1}, y_{2}}^{t-\frac{\Delta t}{2}}-V_{y_{1}, y_{2}}^{t-\Delta t}}{\frac{\Delta t}{2}}\right]+\mathcal{L}_{y_{1}} V^{t-\frac{\Delta t}{2}}+\mathcal{L}_{y_{2}} V^{t-\Delta t}+C F\left(y_{1}, y_{2}\right)
\end{gathered}
$$

Note that (3.24) generate a system of equations for each level of $y_{1}\left(N_{1}\right.$ systems of equations) while (3.25) generates a system of equations for each level of $y_{2}$ ( $N_{2}$ systems of equations). Hence, a full time step requires solving $N_{1}\left(N_{2} \times N_{2}\right)$ tridiagonal systems and $N_{2}\left(N_{1} \times N_{1}\right)$ systems. This requires $\mathrm{O}\left(N_{1} \times N_{2}\right)$ operations to compute. 


\subsection{Numerical Illustration}

\subsubsection{Simulation and Bootstrap}

So far, I have established three models to evaluate agent behaviour using a real options switching model under uncertainty and reversibility. These three models vary in terms of level of complexity and how they compute the cost of switching. The cash-time model is able to evaluate situations where the switching process is time consuming. A prolonged switching process with uncertainty means that agents are not sure if product net income remain in their favour by the time they finish the switching process. If the product net income are not according to what they plan, they may miss the current cash flows and end up in an undesirable situation. At the same time, having the option to reverse the switching process part way through is also uncertain due to the time taken to re-establish the old product. The cash-time model has the advantage of being able to capture all these impacts in terms of the time cost along with the cash cost of switching. The projection model is an approximation of the cash-time model, and captures the time cost partially compared with the cash-time model. In the projection model, switching occurs very fast and agents do not need to wait for a long time but the waiting cost is captured partially in terms of a lump sum cash cost to be paid when switching occurs. When the time of switching increases, the projection model fails to capture the exact cost of switching and underestimates the cost due to this approximation. Finally, the cash-cost model is the simplest model, it captures only the cash cost of switching, and neglects the time cost. Now, the question is: to what extent these three models are different from each other? In order to answer this question, I employ simulation and bootstrapping techniques to simulate a large number of agents and evaluate their behaviours and polices in each model. Then, I test if the results of these models are qualitatively / quantitatively different from each other. I examine agents' behaviour in different 
scenarios and test if these three models are statistically different from each other.

I generate a simulation of 65000 paths over 200 years for two competing products. ${ }^{1}$ This net income simulation helps examine agents' switching behaviour and evaluate when a threshold is crossed to trigger a switch, when they hit the barriers, and how many times they change the state of activity. I use equation (3.26) to generate the product net income processes.

$$
\begin{aligned}
& \Delta \log S_{1}=\left(\mu_{1}-\frac{\sigma_{1}^{2}}{2}\right) \Delta t+\sigma_{1} \epsilon_{1} \\
& \Delta \log S_{2}=\left(\mu_{2}-\frac{\sigma_{2}^{2}}{2}\right) \Delta t+\sigma_{2}\left(\rho_{12} \epsilon_{1}+\sqrt{1-\rho_{12}^{2}} \epsilon_{2}\right)
\end{aligned}
$$

where $\Delta \log S_{i}$ are the changes in log net income of each product, $\mu_{i}$ are the drifts or expected cash flow growths of each product, $\sigma_{i}$ are the volatilities of each product, $\Delta t$ is the time step, $\rho$ is the correlation between the two products, and $\epsilon_{i}$ is a normal random innovation. The $\epsilon_{1}$ and $\epsilon_{2}$ are independent and are normally distributed with a mean and variance of $N(0, \sqrt{\Delta t})$.

Another useful technique that I exploit through my analysis is bootstrapping to test how the three models are significantly different from each other. I solve my optimisation problem according to baseline parameters and examine the behaviour of agents in terms of switching frequency in each model. Then I change my baseline parameters individually and compare the switching frequency across my three models as well as between before and after changes (see Sections 3.3.3 and 3.3.4). In this regard, I find the switching pattern of agents in different scenarios. I generate 10000 random samples with replacement based on my main results in each sce-

\footnotetext{
${ }^{1}$ The choice of 65000 paths and 200 years is ad hoc and can be any number. The life of 200 years might be a long time and a small number e.g. 40 years looks more realistic. The life of 40 years generates less switching and small probabilities that require scaling for a bigger numbers. This can be approximated by dividing the results by 5 .
} 
nario, which has the same size of 65000 observations. This resampling enables us to test whether the mean number of switches, the probability of one or more switches taking place, and the probability of two or more switches taking place are quantitatively/qualitatively different across my three models in each scenario. ${ }^{1}$

\subsubsection{Baseline Parameters and Scenarios}

I test my theory by proposing some baseline parameters. In this regard, Table 3.1 presents a set of reasonable numbers for my optimisation of enterprise allocation. In Section 3.3.4, I examine the sensitivity analysis and the effect of changes in each variable on switching. I evaluate how changes in input parameters may affect switch frequency across the three models and compared with baseline. The time cost changes according to the type of product. For example, in livestock farming (e.g. beef and sheep) there is almost no time cost, and I expect all three models to provide similar results, while with seasonal products (e.g. beetroot and carrot) there is a time cost of one year, and in horticulture (e.g. apples and grapes) the time cost increase to 5 or 6 years, which is a crucial time cost and I expect that the three models to lead to qualitatively different results. My modeling approach allows me to investigate these different cases under one general framework and to carry out counterfactual analyses.

According to my baseline parameters in Table 3.1, it costs $\$ 1000$ and takes 1 year to remove each product, whereas it costs $\$ 10000$ and takes 3 years to initialise Product 1 has a $10 \%$ volatility with $1 \%$ drift while product 2 has $12 \%$ volatility with $1 \%$ drift. These two products have correlation of 0.3 in a market with $5 \%$ discount rate. In Section 3.3.3, I use these parameters to solve my models for the value of the enterprise, extract the optimal

\footnotetext{
${ }^{1}$ I choose $\mathrm{P}(n \geqslant 1)$ and $\mathrm{P}(n \geqslant 2)$ due to a very few observation and small probability for numbers greater than 2 .
} 
TABLE 3.1: Benchmark and Sensitivity Analysis Scenarios

Note: Panel A displays the baseline parameters for two competing products in my analyses. The variables of interest are: cost, time, volatility, drift, discount rate, and correlation. In Panel B, I present the sensitivity analysis in four scenarios: Scenario 1 changes in cost and time, Scenario 2 changes in volatility and drift, Scenario 3 changes in discount rate, and Scenario 4 changes in correlation. Variables are scaled based on thousands (1000) per hectare and time variables are per annum. The sign + indicates that an increase in the variable would be expected to cause arise switching; - indicates that an increase in the variable causes the switching to decrease, and ? indicates that the effect is not clear.

\begin{tabular}{lcccc}
\hline Parameters & Symbol & Product 1 & Product 2 & Effect \\
\hline Panel A: Baseline Variables & & & & \\
Cost (removal, initialisation) & $K_{i j}$ & $(1,10)$ & $(1,10)$ & \\
Time (removal, initialisation) & $T_{i j}$ & $(1,3)$ & $(1,3)$ \\
Volatility & $\sigma_{i}$ & 0.10 & 0.12 \\
Drift & $\mu_{i}$ & 0.01 & 0.01 \\
Discount rate & $r$ & 0.05 & \\
Correlation & $\rho$ & 0.3 & \\
\hline
\end{tabular}

Panel B: Sensitivity Analysis

Scenario 1: Change in Cost and Time
1.A. Cost (removal, initialisation)
$K_{i j}$
$(10,100)$
$(10,100)$
1.B. Time (removal, initialisation)
$T_{i j}$

\section{Scenario 2: Change in Volatility and Drift}

2.A. Volatility
2.B. Drift

Scenario 3: Change in Discount Rate

3.A Discount rate

$\begin{array}{ll}\sigma_{i} & 0.16 \\ \mu_{i} & 0.04\end{array}$

0.18

0.04

Scenario 4: Change in Correlation

4.A. Correlation

$\begin{array}{ll}\rho & +0.8 \\ & -0.8\end{array}$


exercise frontier, and finally examine the frequency of switching in three models. Panel B in Table 3.1 shows four alternative scenarios that I examine in Section 3.3.4 in detail. In each scenario, I change one parameter at a time and evaluate its impact on the agents' optimal switching behaviour. Therefore, I evaluate different scenarios by having simulated GBM paths of product net income from equation (3.26). I begin my analysis by evaluating the behaviour of agents according to the baseline parameters from Table 3.1. Agents in my simulation start in state 1, and, in response to changes in product net income they switch their state of activity. These results provide a benchmark for further analysis when the underlying parameters change.

\subsubsection{Optimal Exercise Frontiers}

In this section, for a given set of parameters, I solve the valuation problem to find how much the enterprise is worth, calculate the optimal strategy, and finally, run a Monte Carlo simulation to see how often agents switch between products over an extended period. In this regard, I calibrate my proposed theory with a set of reasonable numbers from Table 3.1 to quantify enterprise redevelopment in three models: the cash-cost model, the projection model, and the cash-time.

Table 3.2 shows the mean number of switches, the probability of one or more switches taking place, and the probability of two or more switches taking place, based on 65000 simulation paths over 200 years. I evaluate the switching behaviour between two competing products in three different models. In this regard, there will be some combinations where there is no switch in either direction. Also, there are some simulations where there is a switch from product 1 to product 2 but not a switch from product 2 to product 1 . Finally, there are some simulations where multiple switches take place. The magnitude of switches in our results may indicate infre- 
TABLE 3.2: Benchmark Switching Analysis

Note: This table presents the mean number of switches, the probability of one or more switches taking place, and the probability of two or more switches taking place, based on 65000 simulation paths over 200 years. The choice of 65000 and 200 is ad hoc and it should be enough to evaluate the behaviour of agents. I bootstrap 10000 random samples with the same size of 65000 using sampling with replacement. I use this technique to test if the statistics of the mean number of switches, the probability of one or more switches taking place, and the probability of two or more switches taking place (in the projection model vs the cash-cost model and the cash-time model vs the projection model) are quantitatively different. The superscripts ${ }^{* * *}, * *$, and * indicate significance at the $1 \%, 5 \%$, and $10 \%$ level respectively.

\begin{tabular}{lccc}
\hline Variable & Cash-Cost Model & Projection Model & Cash-Time Model \\
\hline \multicolumn{2}{l}{ Baseline Parameters Analysis } & & \\
Mean & 1.6049 & $1.0072^{* * *}$ & $0.9621^{* * *}$ \\
$\mathrm{P}(n \geqslant 1)$ & 0.8233 & $0.7551^{* * *}$ & $0.7425^{* * *}$ \\
$\mathrm{P}(n \geqslant 2)$ & 0.3914 & $0.2011^{* * *}$ & $0.1804^{* * *}$ \\
\hline
\end{tabular}

quent switches, due to the costly process of switching. However, the interest of this section is to emphasise the difference between the three models of switching no matter if they are different with frequent or infrequent switches. An important contribution of evaluating underlying factors in different scenarios is to introduce more relevant model of valuation according to different problems. Also, this differentiation among the three models helps to avoid underestimating or overestimating the switching of a problem.

Table 3.2 presents significance level for tests for difference in the reported statistics between the cash-cost and projection models as well as between the projection and cash-time models. In all cases, I reject the null of no difference at the $1 \%$ significance level. However, the difference between the cash-cost and projection models are quantitatively larger than the difference between the projection and cash-time models. The difference arises due to the measure of cost effect in each model. The cash-cost model takes into account only the cash cost of switching, whereas the pro- 
jection model considers cash cost along with the expected monetary cost of time (in terms of forgone profits) and the cash-time model considers the cash cost along with the exact time cost. In other words, the cash-cost model captures only the cash cost whereas the projection and cash-time models capture not only the cash cost but also the time cost, (albeit, with different accuracy). In Section 3.3.4, I evaluate the changes in my baseline parameters, the effect on switch frequency, and the underlying reasons for my results.

The difference between the three models arises from the complexity of calculating switching cost as the net income of each product changes over the course of time. According to the set up and constraints, each model will have a different set of optimal exercise frontiers (OEFs). The OEFs trace the locus of product net income levels that will trigger an agent to switch products. The OEFs enable the models to provide normative advice on how enterprise should be used (i.e. when products should be changed). Figure 3.1 compares the OEFs for the three models according to baseline parameters in Table 3.1. Note that the OEFs for the projection model and the cash-time model are not qualitatively very different in Figure 3.1 due to the baseline parameters. Once I change the time taken to switch (see Section 3.3.4), I see the projection and cash-time model OEFs start to diverge. In other words, formal treatment of time, when time is a larger part of the cost, is very important.

Figure 3.1 displays the optimal exercise frontiers for two competing products in three models: the cash-cost model with the dotted lines, the projection model with the dash-dotted lines, and the cash-time model with the solid lines. The horizontal axis shows the product 1 net income and the vertical axis shows the product 2 net income. The area above the lower solid line in the cash-time model indicates no switch from product 2, whereas the area below the upper solid line shows no switch from product 1 , and the area between two boundaries (solid lines) is an inactive 


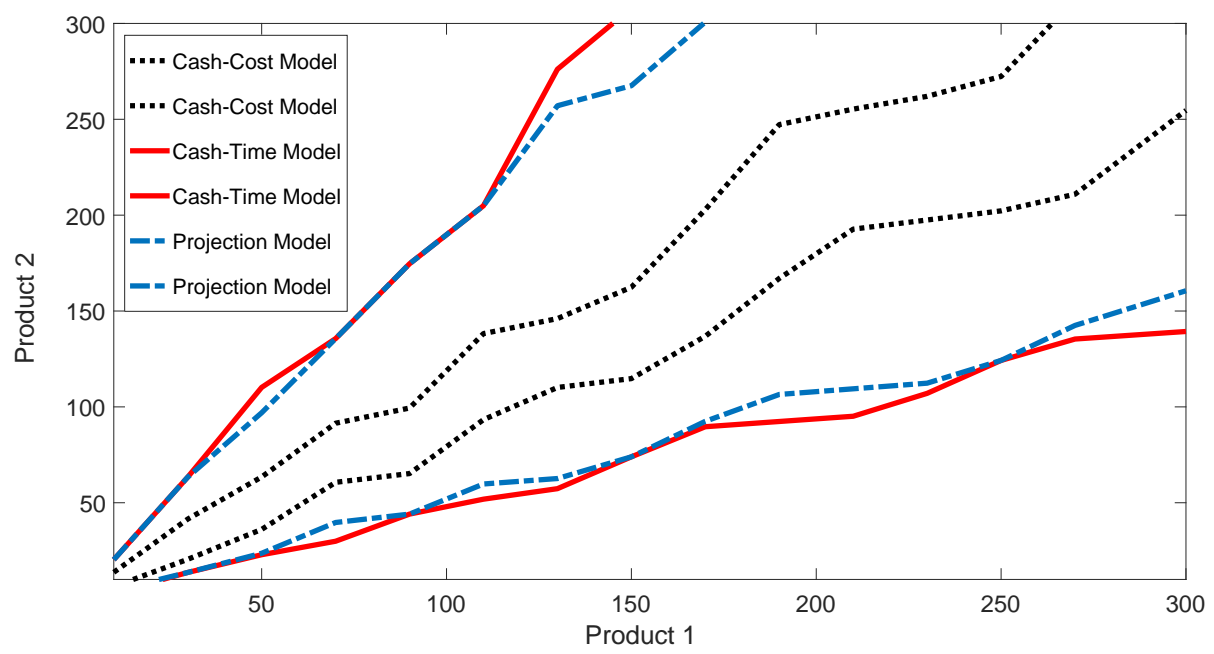

FIGURE 3.1: Optimal Exercise Frontier for Baseline Parameters.

Note: This figure displays the optimal exercise frontiers for two competing products in three models: the cashcost model with the dotted lines, the projection model with the dash-dotted lines, and the cash-time model with the solid lines. The horizontal axis shows the product 1 net income and the vertical axis shows the product 2 net income. The area above the lower solid line in the cash-time model indicates no switch from product 2, whereas the area below the upper solid line shows no switch from product 1 , and the area between two boundaries (solid lines) is an inactive region that agents will not start transition. Similarly, the same area can be defined for the cash-cost and projection models according to their boundaries, which are the dotted lines and the dash-dotted lines respectively. Note that the cash-cost model has the lowest gap among all three models due to capturing only the cash cost. The projection model is a good approximation of the cash-time model in this case because they are under the baseline parameters. The cash-time model starts to have a higher gap than the projection model when the time of switching increases and agents should go through a prolonged switching processes under uncertainty and reversibility.

region with no switch and agents will not start transition. Similarly, the same area can be defined for the cash-cost and projection models according to their boundaries, which are the dotted lines and the dash-dotted lines respectively.

The cash-cost model is oversimplified and agents would be expected to switch more often because they only need to pay a cash cost to switch (loss of product net income is ignored). Meanwhile, the projection model with the dash-dotted lines provides less chance for agents to switch due to accounting for the loss of product net income during the switch. In other words, agents should not only pay the cash cost in the cash-cost model but also pay a cost that proxies for the time cost, and is related to the current product values (see equation (3.12)). This opportunity cost is cheap for 
products that are not worth much, but very expensive for products that are lucrative. Finally, the cash-time model, with the solid lines, fully and formally takes into account the time cost and has exercise frontiers with a wider gap. The cash-time model, compared with the other two models, makes it even harder to switch due to the extra time cost compared with the cash-cost model (and full time cost compared with the projection model that partially incorporates the time cost). Agents in the cash-time model have the option to abort the switching process and go back to the original product. However, they have to pay a cash cost and wait for a while to resume their initial activity. What makes the prolonged switching process more uncertain and perilous is that by the time agent complete the transition, cash flows may have changed substantially. Comparing all three models show that the time cost is proportional to product values whereas the cash cost does not scale according to product net income. Having said that, the OEFs in all three models are close to each other when product net income are low but further apart when the product net income are high. It is important to note that any changes in underlying parameters will cause the OEFs to get closer or move apart. For example, as the transition time increases, the gap between the projection and cash-time models increases more (see Figure 3.3), which is not significant with my baseline parameters in Figure 3.1. The projection model is much simpler and computationally cheaper for situations where the switching process is not so prolonged. Hence, the projection model leads us into implementing all types of analyses much easier. Conversely, when the transition time gets longer and we are not in the baseline scenario, the cash-time model is the most comprehensive model for my analyses and the results will be significantly different from the cash-time model.

The jaggedness of the OEFs is due to the discreteness in my finite difference grids. In other words, I discretise the PDE problem and solve it on specific product net income combinations, which are not continuous. Therefore, as I increase the number of steps and make my discrete points 
closer, I will achieve smoother lines. The caveat is that the higher number of net income steps will increase the computational cost of solving the problem. Having said that, in some cases, for example, when I have negative correlation I will achieve smooth lines rather than jagged lines. The main reason for the jaggedness is the use of the transformation (see equation (3.16)). The negative correlation affects the covariance matrix and results in smooth lines due to using a different transformation. Figure 3.2 is an example of this situation using the parameters for my negative correlation sensitivity analysis in Section 3.3.4 (see Table 3.1). Figure 3.2 shows

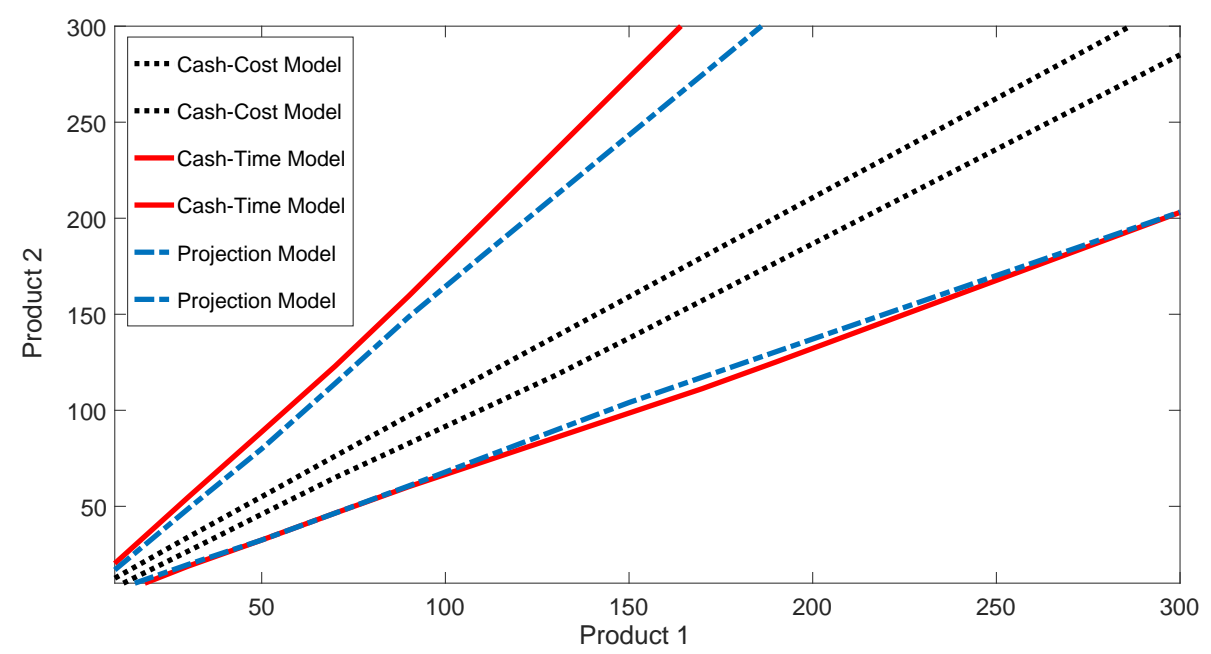

FIGURE 3.2: Optimal Exercise Frontier for Baseline Parameters but Negative Correlation.

Note: This figure displays the optimal exercise frontiers for two competing products in three models: the cashcost model with the dotted lines, the projection model with the dash-dotted lines, and the cash-time model with the solid lines. The horizontal axis shows the product 1 net income and the vertical axis shows the product 2 net income. I note that the cash-cost model has the lowest gap among all three models due to capturing only the cash cost. The projection model is a good approximation of the cash-time model in this case because they are under the baseline parameters. The cash-time model starts to have a higher gap than the projection model when the time of switching increases and agents should go through a prolonged switching processes under uncertainty and reversibility. This figure how the jaggedness of figures disappear when I have a negative correlation. The reason refers to using eigenvalues in my transformation where the negative correlation affects the covariance matrix and results in smooth lines due to different transformation..

OEFs with smooth lines for three different models: the cash-cost model with the dotted lines, the projection model with the dash-dotted lines, and finally the cash-time model with solid lines. 


\subsubsection{Sensitivity Analysis}

\section{Cost and Time Sensitivity Analysis}

In this section, I analyse the likelihood of switching when the baseline parameters change, as shown in Table 3.1. In this regard, I evaluate the impact of an increase in cost and time in two separate scenarios. In Scenario 1.A, I change the total cost of removing from $\$ 1000$ per ha to $\$ 10000$ per ha and the total cost of initialisation from $\$ 10000$ per ha to $\$ 100000$ per ha. In Scenario 1.B, I increase the time of removing from 1 year to 10 years and the time of initialisation from 3 years to 8 years. Then, in each scenario, I compare the switching frequency across my three models. Table 3.3 demonstrates the mean number of switches, the probability of one or more switches taking place, and the probability of two or more switches taking place across my three models due to an increase in cost (Scenario 1.A) and time (Scenario 1.B) separately.

Table 3.3 reports the baseline parameter results for benchmark, Scenario 1.A (dollar cost increases), and Scenario 1.B (time cost increases). When I increase the cost of switching, I expect all three models to be affected by this effect, because all three models take into account the cash cost. I note that the mean number of switches, the probability of one or more switches taking place, and the probability of two or more switches taking place, all decrease compared with my benchmark results. In other words, an increase in the cash cost makes switching expensive and pushes the OEFs further out, which in turn results in less switching. The difference between the cash-cost and projection models as well as the difference between the projection and cash-time models are significant at the $1 \%$ level, but the magnitude of the differences are not very large. However, in Scenario 1.B, where I increase the time cost (duration of switch) I note that the difference between model pairs starts to widen a lot more than when I change dollar costs. 
TABLE 3.3: Cost and Time Switching Analysis

Note: This table presents the mean number of switches, the probability of one or more switches taking place, and the probability of two or more switches taking place based on 65000 simulation paths over 200 years. The choice of 65000 and 200 is ad hoc and it should be enough to evaluate the behaviour of agents. I use bootstrap method to generate 10000 random samples with the same size of 65000 and replacement. I use this technique to test if the statistics of the mean number of switches, the probability of one or more switches taking place, and the probability of two or more switches taking place in the projection model vs the cash-cost model and the cash-time model vs the projection model are quantitatively/qualitatively different. The superscripts ${ }^{* * *}, * *$, and ${ }^{*}$ indicate significance at the $1 \%, 5 \%$, and $10 \%$ level respectively.

\begin{tabular}{lccc}
\hline Variable & Cash-Cost Model & Projection Model & Cash-Time Model \\
\hline \multicolumn{2}{l}{ Baseline Parameters Analysis } & & \\
Mean & 1.6049 & $1.0072^{* * *}$ & $0.9621^{* * *}$ \\
$\mathrm{P}(n \geqslant 1)$ & 0.8233 & $0.7551^{* * *}$ & $0.7425^{* * *}$ \\
$\mathrm{P}(n \geqslant 2)$ & 0.3914 & $0.2011^{* * *}$ & $0.1804^{* * *}$ \\
\hline Scenario 1.A: Change in Cost & & \\
Mean & 0.6342 & $0.5674^{* * *}$ & $0.4765^{* * *}$ \\
$\mathrm{P}(n \geqslant 1)$ & 0.5484 & $0.5225^{* * *}$ & $0.4524^{* * *}$ \\
$\mathrm{P}(n \geqslant 2)$ & 0.0702 & $0.0412^{* * *}$ & $0.0227^{* * *}$ \\
\hline Scenario 1.B: Change in Time & & \\
Mean & 1.6049 & $0.6480^{* * *}$ & $0.5330^{* * *}$ \\
$\mathrm{P}(n \geqslant 1)$ & 0.7383 & $0.5895^{* * *}$ & $0.5100^{* * *}$ \\
$\mathrm{P}(n \geqslant 2)$ & 0.2506 & $0.0551^{* * *}$ & $0.0225^{* * *}$ \\
\hline
\end{tabular}


Scenario 1.B reports that an increase in switching time decreases the mean number of switches, the probability of one or more switches taking place, and the probability of two or more switches taking place in all three models (compared to my results in the benchmark). The difference across my three models are statistically significantly different at the $1 \%$ level. However, I expect that an increase in time effect provides a larger difference between my competing models when time is a much bigger part of the cost. In this case, the projection model is different from the cash-cost model due to larger time cost and the cash-time model is different from the projection model due to its more formal treatment of time, which becomes more important when the conversion process is longer. The projection model implies that an increase in the time cost leads to a higher approximation of opportunity cost that agents should pay, along with the cash cost. The cash-time model implicitly captures the opportunity cost by letting agents be in a "Transition" state where no products are produced. The longer the time, the bigger the difference gets between the projection and cash-time models. I demonstrate the optimal policies under Scenario 1.B for my three models in Figure 3.3.

Figure 3.3 shows the OEFs for three models. The cash-cost model is displayed with the dotted lines, the projection model with dash-dotted lines, and finally the cash-time model with solid lines. Figure 3.3 indicates that when the switching process entails more time, the optimal polices for the projection model and the cash-time model start to diverge and become significantly different from each other, but it has little effect on the cashcost model (compared with Figure 3.1). The reason is that the cash-cost model captures only the cash cost and time of switching is not involved whereas the projection and cash-time models capture both, and the time cost becomes more crucial as the time taken for switching increases. On the one hand, agents could wait for a long time to complete the process and miss the current cash flows. On the other hand, uncertainty of product net income makes agents reluctant to exercise their options because 


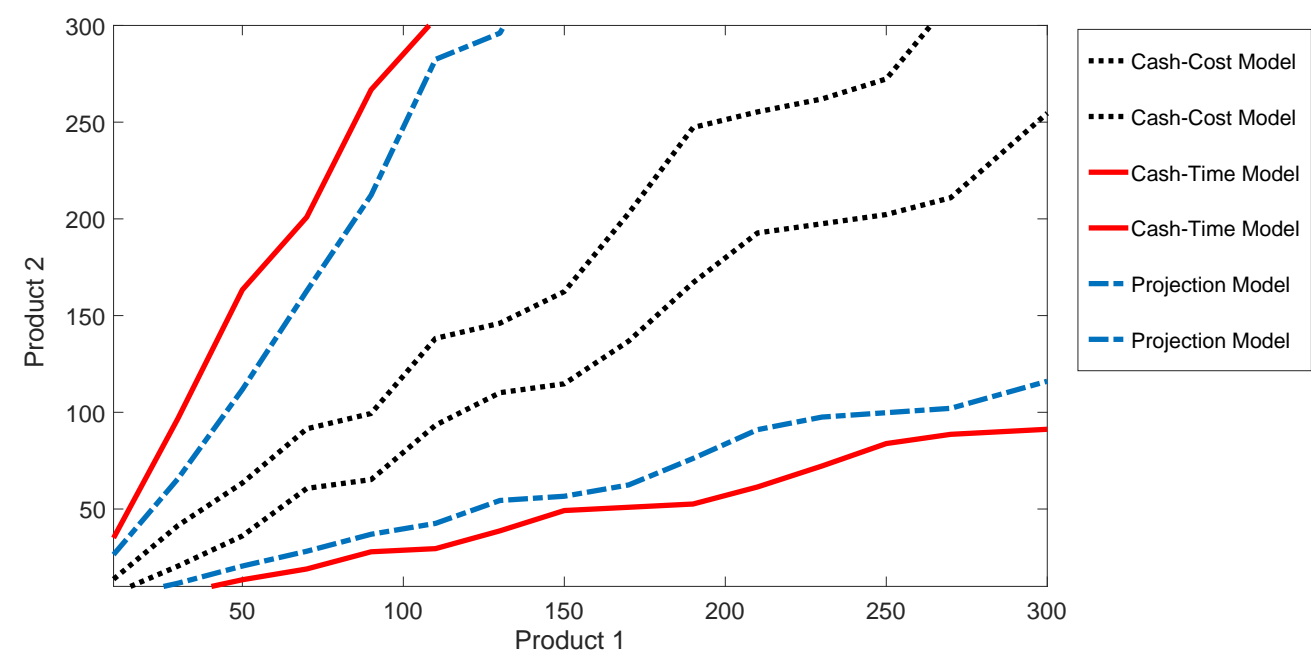

FIGURE 3.3: Optimal Exercise Frontier when Time Changes (Scenario 1.B).

Note: This figure shows the optimal exercise frontiers for three models. The cash-cost model is displayed with the dotted lines, the projection model with dash-dotted lines, and finally the cash-time model with solid lines. The horizontal axis displays the product 1 net income and the vertical axis displays product 2 net income. The cash-cost model has the lowest gap of optimal polices due to capturing only the cash cost. The projection model and the cash-time model have the higher gap compared with the cash-cost model due to capturing the cash cost and the time cost. However, the projection model is an approximation of the time cost whereas the cash-time model is the exact time cost. I note that in prolonged switching processes the cash-time model is the competent model.

they are not sure what product net income they will end up at the end of process and even if they reverse the process part way through it will not be quick to go back to the existing activity. Hence, the projection model approximates the impact of prolonged cost of switching whereas the cashtime model computes the exact time cost and results in wider optimal polices compared with the projection model. Overall, an increase in cost and time both have a negative impact on the mean number of switches and the probability of switches but the magnitude of changes varies according to the scenario and type of model.

\section{Volatility and Drift Sensitivity Analysis}

In this section, I change volatility and drift values for the net income process. Therefore, I evaluate the impact of each variable separately. In Sce- 
TABLE 3.4: Volatility and Drift Switching Analysis

Note 1: This table presents the mean number of switches, the probability of one or more switches taking place, and the probability of two or more switches taking place based on 65000 simulation paths over 200 years. The choice of 65000 and 200 is ad hoc and it should be enough to evaluate the behaviour of agents. I use bootstrap method to generate 10000 random samples with the same size of 65000 and replacement. I use this technique to test if the statistics of the mean number of switches, the probability of one or more switches taking place, and the probability of two or more switches taking place in the projection model vs the cash-cost model and the cash-time model vs the projection model are quantitatively/qualitatively different. The superscripts ${ }^{* * *}, * *$, and ${ }^{*}$ indicate significance at the $1 \%, 5 \%$, and $10 \%$ level respectively.

\begin{tabular}{lccc}
\hline Variable & Cash-Cost Model & Projection Model & Cash-Time Model \\
\hline \multicolumn{2}{l}{ Baseline Parameters Analysis } & & \\
Mean & 1.6049 & $1.0072^{* * *}$ & $0.9621^{* * *}$ \\
$\mathrm{P}(n \geqslant 1)$ & 0.8233 & $0.7551^{* * *}$ & $0.7425^{* * *}$ \\
$\mathrm{P}(n \geqslant 2)$ & 0.3914 & $0.2011^{* * *}$ & $0.1804^{* * *}$ \\
\hline Scenario 2.A: Change in Volatility & & \\
Mean & 1.5342 & $0.9465^{* * *}$ & 0.9416 \\
$\mathrm{P}(n \geqslant 1)$ & 0.8107 & $0.6938^{* * *}$ & 0.6938 \\
$\mathrm{P}(n \geqslant 2)$ & 0.3597 & $0.1920^{* * *}$ & 0.1941 \\
\hline Scenario 2.B: Change in Drift & & \\
Mean & 2.4251 & $0.9016^{* * *}$ & $0.7171^{* * *}$ \\
$\mathrm{P}(n \geqslant 1)$ & 0.8606 & $0.6862^{* * *}$ & $0.5949^{* * *}$ \\
$\mathrm{P}(n \geqslant 2)$ & 0.5320 & $0.1729^{* * *}$ & $0.1067^{* * *}$ \\
\hline
\end{tabular}

nario 2.A, I increase the volatility of product 1 from $10 \%$ to $16 \%$ and product 2 from $12 \%$ to $18 \%$ and examine the results. In Scenario 2.B, I set back everything and increase the drift of product 1 from $1 \%$ to $4 \%$ and product 2 from $1 \%$ to $4 \%$ and see what is the impact of drift this time. Table 3.4 presents the results for changes and the corresponding impacts on the mean number of switches, the probability of one or more switches taking place, and the probability of two or more switches taking place. 
Table 3.4 shows the baseline parameter results as the benchmark, Scenario 2.A (increased volatility), and Scenario 2.B (increased drift). Scenario 2.A reports that an increase in volatility has a negative impact on the three measures of interest. In other words, my results in Scenario 2.A are quantitatively less than baseline, except the probability of two or more switches taking place in the cash-time model, which increases. The results across the three models show that the difference between the cash-cost and projection models is large and significant at the $1 \%$ level whereas the difference between the projection and cash-time models is almost zero and not significant. In this case, my results suggest that the projection model is a good approximation of the cash-time model. The projection model captures the impact of an increase in volatility by measuring the expected changes of the product net income as an extra cost that decreases switching compared with the cash-cost model. In contrast, the cash-time model measures the volatility as uncertainty in product net income that has a negative impact on switching. Agents in the cash-time model are concerned that product net income might move against their favour while they are in the process of removing and initialisation or even when they abort the switching process part way through to go back to their existing activity.

A higher volatility provides more time value to the options, and results in agents being more reluctant to exercise their options. In other words, agents are able to profit in the cash-time model because they get more value from uncertainty but the value of the option to switch products is so high that agents become reluctant to exercise their options. For the time being, I focus on the enterprise value of the cash-time model. To illustrate this point, I display the enterprise value when agents produce product 1 in Figure 3.4.

Figure 3.4 demonstrates the enterprise value with product 1 in the cash-time model. The enterprise value increases in both dimensions be- 


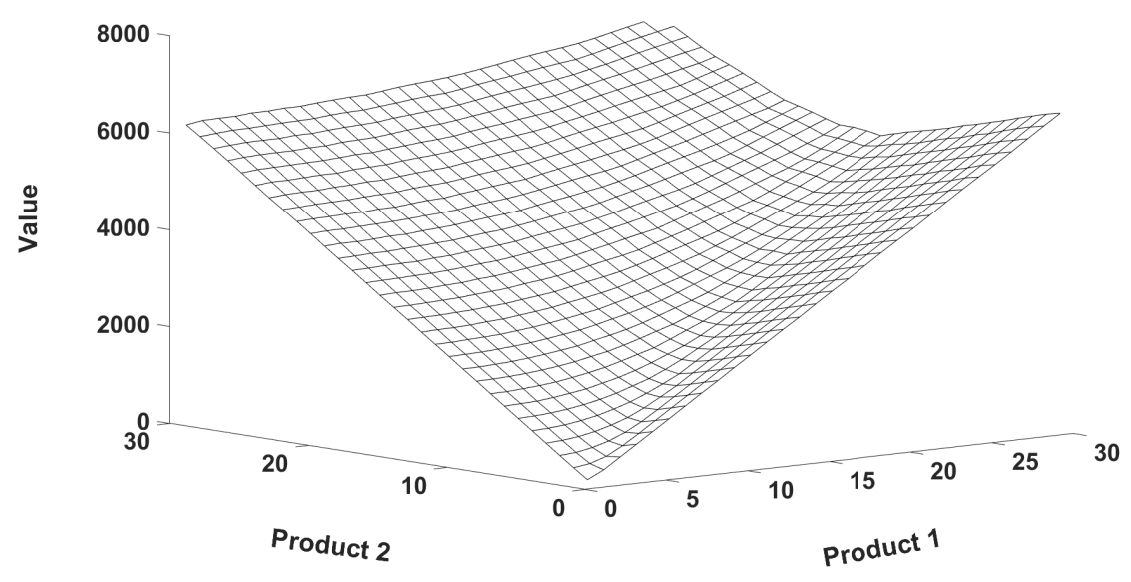

FIGURE 3.4: Enterprise Value with Product 1 in the cash-time model when volatility increases.

Note: This figure shows the enterprise value in the cash-time model, producing product 1 when volatility increases in the baseline parameters. The enterprise value is on the $z$ axis and the product net income are on the $x$ and $y$ axes. I show how agents switch from producing one type of product to another when the product net income change over the course of time.

cause either the product that the agent is producing is worth more or the option to switch to the competing product is producing in value. I observe that the enterprise value is curved in the middle due to the time value of the option to switch. The enterprise value is convex, which means there is option value. To better illustrate this convexity, I plot the secondary diagonal (lower left entry to the upper right entry) of enterprise values producing products 1 and 2 before (baseline) and after changes in volatility, which is displayed in Figure 3.5.

Figure 3.5 shows a cross-section of enterprise values producing products 1 and 2 . The vertical axis displays the enterprise values and the horizontal axis displays the secondary diagonal of matrix values for each enterprise. The values under the baseline parameters are displayed with the dotted curve and under Scenario 2.A with the solid curves. Also, enterprises producing product 1 are displayed with the circle marker and enterprises producing product 2 with the star marker. All the values are evaluated using the cash-time model, because this model is able to evalu- 


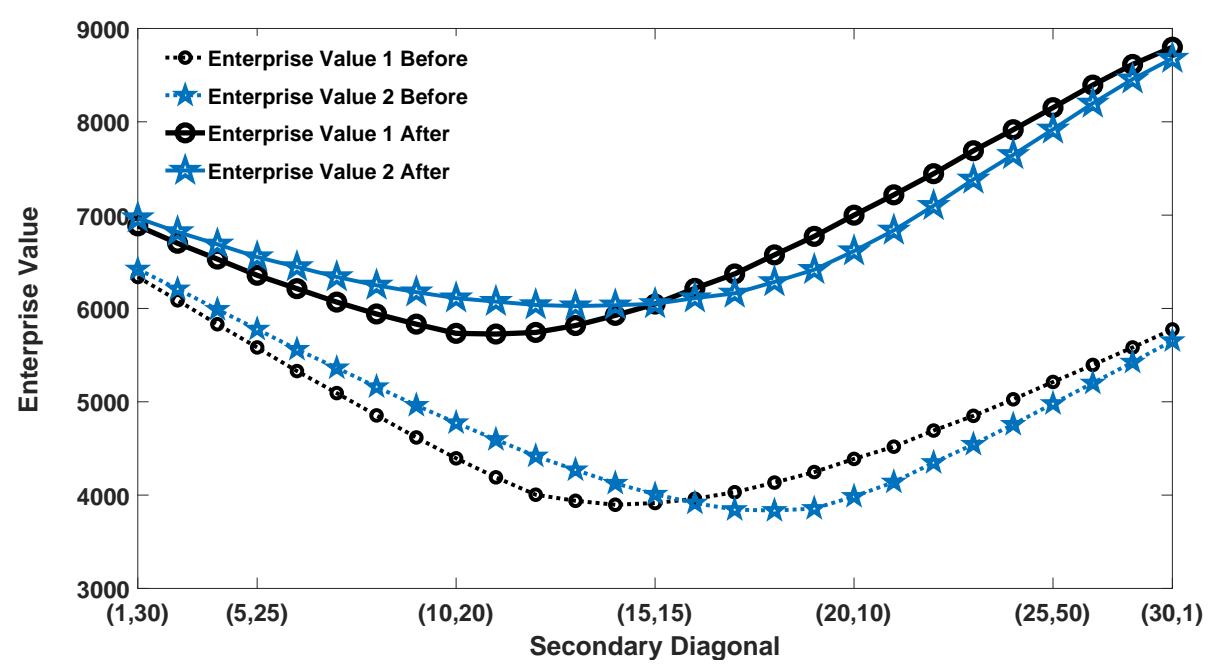

FIGURE 3.5: Enterprise Values Before (baseline) and After Change in Volatility in the cash-time model.

Note: This figure shows the cross section of enterprise values producing products 1 and 2 . The horizontal axis shows the secondary diagonal of matrix values and the vertical axis show the corresponding values of each coordinates. The values under the baseline parameters are displayed with the dotted curve and under Scenario 2.A with the solid curves. Also, enterprises producing product 1 are displayed with the circle marker and enterprises producing product 2 with the star marker.

ate prolonged real options to switch problems under uncertainty and reversibility and capture the exact switching cost. An increase in volatility shifts the curve up and to the left, and offers more option value. Higher volatility results in more uncertainty, but at the same time adds convexity to the curve that provides value to the switching option. In Figure 3.5, I observe that the right part of curve gains more option value than the left part when the volatility goes up. In other words, one of the products is really in the money and gives a higher value than the competing product and by exercising the switching option I can obtain a higher value.

Scenario 2.B in Table 3.4 reports the results for a change in drift. When the drift goes up, the mean number of switches, the probability of one or more switches taking place, and the probability of two or more switches taking place in the cash-cost model increases (compared with the baseline) whereas they decrease in the projection model and the cash-time model. One explanation is that when drift goes up, agents will gain from the product returns due to the growth rate that convince them to switch. 
In contrast, the drift (growth rate) is not enough to cover both the cash cost and the time cost in the projection and cash-time models. My results across my three models display that the difference between all my measures, comparing the cash-cost model and the projection model as well as comparing the projection model and the cash-time model, are significantly different at the $1 \%$ level. However, the actual difference between the cash-cost and projection models is larger than the difference between the projection and cash-time models. Overall, comparing the impact of drift and volatility may indicate that switches are more sensitive to drift than volatility. As I explained, the reason is that volatility has an option value effect (see Figure 3.5) that reduces the impact of an increase in volatility whereas a change in drift has a fundamental effect on values through discounted dividend model (DDM) that discounts the values by the difference between discount rate and $\operatorname{drift}\left(C F_{i} /\left(\mu_{i}-r\right)\right)$.

\section{Discount Rate Sensitivity Analysis}

In this section, I examine the impact of an increase in the discount rate on the frequency of switching. Therefore, I increase the discount rate from $5 \%$ to $12 \%$ and compare the results with my benchmark and across my three models. Table 3.5 presents the results for changes in switching frequency for agents when I increase the discount rate.

Table 3.5 shows that when the discount rate goes up, the mean number of switches, the probability of one or more switches taking place, and the probability of two or more switches taking place, decreases in all three models. Changes in the discount rate have a two opposing effects and the outcome depends on which effect dominates. On the one hand, when the discount rate goes up, the time value of the option (regardless of the option type) goes down, and agents become impatient and prefer to pull the trigger and switch to the more profitable product. In other words, the value of future cash flows may not be worth as much as before and agents 
TABLE 3.5: Discount Rate Switching Analysis

Note 1: This table presents the mean number of switches, the probability of one or more switches taking place, and the probability of two or more switches taking place based on 65000 simulation paths over 200 years. The choice of 65000 and 200 is ad hoc and it should be enough to evaluate the behaviour of agents. I use bootstrap method to generate 10000 random samples with the same size of 65000 and replacement. I use this technique to test if the statistics of the mean number of switches, the probability of one or more switches taking place, and the probability of two or more switches taking place in the projection model vs the cash-cost model and the cash-time model vs the projection model are quantitatively/qualitatively different. The superscripts ${ }^{* * *}, * *$, and ${ }^{*}$ indicate significance at the $1 \%, 5 \%$, and $10 \%$ level respectively.

\begin{tabular}{llll}
\hline Variable & Cash-Cost Model & Projection Model & Cash-Time Model \\
\hline
\end{tabular}

\section{Baseline Parameters Analysis}

$\begin{array}{llll}\text { Mean } & 1.6049 & 1.0072^{* * *} & 0.9621^{* * *} \\ \mathrm{P}(n \geqslant 1) & 0.8233 & 0.7551^{* * *} & 0.7425^{* * *} \\ \mathrm{P}(n \geqslant 2) & 0.3914 & 0.2011^{* * *} & 0.1804^{* * *}\end{array}$

\section{Scenario 3: Change in Discount Rate}

$\begin{array}{llll}\text { Mean } & 1.5366 & 0.9328^{* * *} & 0.6283^{* * *} \\ \mathrm{P}(n \geqslant 1) & 0.8124 & 0.7350^{* * *} & 0.5739^{* * *} \\ \mathrm{P}(n \geqslant 2) & 0.3700 & 0.1661^{* * *} & 0.0510^{* * *}\end{array}$


prefer to switch as soon as they get an opportunity that is profitable rather than waiting to see if they can achieve a higher profit. In the extreme case where the discount rate equals infinity, the time value of the option disappears completely and provides a myopic situation where agents prefer to exercise any option in hand at present without any regard for the future. On the other hand, when the discount rate goes up, agents' expectations of future returns tend to increase over the course of time. Hence, when they have any option to switch they are willing to wait and see if it gets even better. My results suggest that the latter effect outweighs the former, which means that an increase in discount rate has a negative impact on switch frequency, and agents prefer to wait more than to switch into an alternative product quickly.

Comparing my results across the three models shows that the mean number of switches, the probability of one or more switches taking place, and the probability of two or more switches taking place decreases from the cash-cost model to the projection model, and from the projection model to the cash-time model. The difference between pair models is significant at the $1 \%$ level. I observe that the mean number of switches and the probability of two or more switches taking place has a larger difference between the cash-cost and projection models than between the projection and cashtime models, whereas the probability of one or more switches taking place has a larger difference between the projection and cash-time models than between the cash-cost and projection models. In other words, an increase in discount rates causes the agents to switch multiple times in the projection model than the cash-cost model and they prefer to have no switches or one switch in the cash-time model relative to the projection model.

\section{Correlation Sensitivity Analysis}

In this section, I examine the changes in correlation between the two competing products, net income. I evaluate the switch frequency when cor- 
TABLE 3.6: Correlation Switching Analysis

Note 1: This table presents the mean number of switches, the probability of one or more switches taking place, and the probability of two or more switches taking place based on 65000 simulation paths over 200 years. The choice of 65000 and 200 is ad hoc and it should be enough to evaluate the behaviour of agents. I use bootstrap method to generate 10000 random samples with the same size of 65000 and replacement. I use this technique to test if the statistics of the mean number of switches, the probability of one or more switches taking place, and the probability of two or more switches taking place in the projection model vs the cash-cost model and the cash-time model vs the projection model are quantitatively/qualitatively different. The superscripts ${ }^{* * *}, * *$, and *indicate significance at the $1 \%, 5 \%$, and $10 \%$ level respectively.

\begin{tabular}{lccc}
\hline Variable & Cash-Cost Model & Projection Model & Cash-Time Model \\
\hline \multicolumn{1}{l}{ Baseline Parameters Analysis } & & \\
Mean & 1.6049 & $1.0072^{* * *}$ & $0.9621^{* * *}$ \\
$\mathrm{P}(n \geqslant 1)$ & 0.8233 & $0.7551^{* * *}$ & $0.7425^{* * *}$ \\
$\mathrm{P}(n \geqslant 2)$ & 0.3914 & $0.2011^{* * *}$ & $0.1804^{* * *}$ \\
\hline Scenario 4.A: Change in Correlation (Positive) & \\
Mean & 1.0568 & $0.5076^{* * *}$ & $0.4378^{* * *}$ \\
$\mathrm{P}(n \geqslant 1)$ & 0.7921 & $0.4990^{* * *}$ & $0.4334^{* * *}$ \\
$\mathrm{P}(n \geqslant 2)$ & 0.1863 & $0.0085^{* * *}$ & $0.0044^{* * *}$ \\
\hline Scenario 4.B: Change in Correlation (Negative) & \\
Mean & 5.5017 & $2.6163^{* * *}$ & $2.5173^{* * *}$ \\
$\mathrm{P}(n \geqslant 1)$ & 0.9468 & $0.9052^{* * *}$ & 0.9060 \\
$\mathrm{P}(n \geqslant 2)$ & 0.7962 & $0.6281^{* * *}$ & $0.6150^{* * *}$ \\
\hline
\end{tabular}

relation between product net income changes from +0.3 to +0.8 or -0.8 . I report my results for changes in correlation in Table 3.6.

Table 3.6 reports the results for my benchmark, Scenario 4.A (a change in correlation in the positive direction), and Scenario 4.B (a change in correlation in the negative direction). Scenario 4.A shows that all three measures decrease when correlation goes up in the positive direction. I postulate that gaps in product net income persist. This means, the cash flows 
from products move together in the same direction, which makes it more likely that if product $\mathrm{A}$ is more lucrative at time $t$ it remains more lucrative after that. Evaluating the results across my three models shows that the difference between the cash-cost and projection models is larger than the difference between the projection and cash-time models, and all my results are significant at the $1 \%$ level.

Scenario 4.B demonstrates that the mean number of switches, the probability of one or more switches taking place, and the probability of two or more switches taking place increase when the correlation is negative. In this case, the cash flows from products move in the opposite direction and when one product becomes less profitable, and the competing product becomes more lucrative. My results across the three models demonstrate that the difference between the cash-cost and projection models is larger than the difference between the projection and cash-time models. My results for the difference across pair models are significant at the $1 \%$ level except for the probability of one or more switches taking place between the projection and cash-time models.

\section{Diff-in-Diff and Relative Value Changes Sensitivity Analysis}

So far, I evaluated the significant changes between three different models (the cash-cost model, the projection model, and the cash-time model) by increasing baseline parameters one at a time. As a follow up, I examine if there is a significant change before and after change compared with the baseline parameters. Table 3.7 presents the summary results for all changes. Results show that the mean and the probability of one or more for all scenarios except the change in time for the cash-cost model are significantly different from the baseline model. The change in time will not affect the mean of the cash-cost model. The probability of two or more for the projection model are significant when there is a change in drift, discount rate and correlation. The probability of two or more for the cash- 
time model are significant when there is a change in cost, discount rate, and negative correlation.

In the following, I evaluate the sensitivity analysis when the relative values are changed compared with the baseline parameters. Table 3.8 reports the baseline results for benchmark, Scenario 5.A (dollar cost increases), Scenario 5.B (time cost increases), Scenario 5.C (volatility increases), and Scenario 5.D (drift increases). All values are increased for product 2 so we can examine the change in relative values. Therefore, the cost of removing product 2 increases from $\$ 1000$ to $\$ 10000$ and its cost to initialise increases from $\$ 10000$ to $\$ 30000$. The time of removing product 2 increases from 1 year to 3 years and its time to initialise increases from 3 years to 6 years. The volatility and drift of product 2 increases from 10 and 0.01 to 14 and 0.04 respectively.

The results for relative changes in Table 3.8 shows that our main results from the previous analysis holds and the projection model is significantly different from the cash-cost model, while the cash-time model is significantly different form the projection model. ${ }^{1}$ When the cost, time, volatility, and drift of product 2 increase, it is more costly to switch from one product to another and the mean number of switches, probability of one or more, and probability of two or more switches drop noticeably. Overall, as we increase the cost, time, volatility, and drift of one product alone, the switching process converges to zero and the level of being harder to switch increases as we move from the cash-cost to projection model and finally to the cash-time model.

\footnotetext{
${ }^{1}$ I test the difference across the three models when the parameters for product 2 change and for product 1 do not change.
} 


\section{CHAPTER 3. OPTIMAL ENTERPRISE USE SWITCHING POLICY}

TABLE 3.7: Changes in the Switching Behaviour When the Baseline Parameters Changes in Each Scenario.

Note 1: This table presents the mean number of switches, the probability of one or more switches taking place, and the probability of two or more switches taking place based on 65000 simulation paths over 200 years. I examine the diff-in-diff significance level between the baseline parameters and each scenario. The superscripts ***,**, and * indicate significance at the $1 \%, 5 \%$, and $10 \%$ level respectively.

\begin{tabular}{lccc}
\hline Variable & Cash-Cost Model & Projection Model & Cash-Time Model \\
\hline \multicolumn{2}{l}{ Baseline Parameters Analysis } & & \\
Mean & 1.6049 & 1.0072 & 0.9621 \\
P $(n \geqslant 1)$ & 0.8233 & 0.7551 & 0.7425 \\
P $(n \geqslant 2)$ & 0.3914 & 0.2011 & 0.1804
\end{tabular}

\begin{tabular}{lrll}
\hline \multicolumn{2}{l}{ Scenario 1.A: } & Change in Cost & \\
Mean & $0.6342^{* * *}$ & $0.5674^{* * *}$ & $0.4765^{* * *}$ \\
$\mathrm{P}(n \geqslant 1)$ & $0.5484^{* * *}$ & $0.5225^{* * *}$ & $0.4524^{* * *}$ \\
$\mathrm{P}(n \geqslant 2)$ & 0.0702 & 0.0412 & $0.0227^{* * *}$ \\
\hline
\end{tabular}

\begin{tabular}{|c|c|c|c|}
\hline \multicolumn{4}{|c|}{ Scenario 1.B: Change in Time } \\
\hline Mean & 1.6049 & $0.6480^{* * *}$ & $0.5330^{* * *}$ \\
\hline $\mathrm{P}(n \geqslant 1)$ & 0.7383 & $0.5895^{* * *}$ & $0.5100^{* * *}$ \\
\hline $\mathrm{P}(n \geqslant 2)$ & 0.2506 & 0.0551 & 0.0225 \\
\hline \multicolumn{4}{|c|}{ Scenario 2.A: Change in Volatility } \\
\hline Mean & $1.5342^{* * *}$ & $0.9465^{* * *}$ & $0.9416^{* * *}$ \\
\hline $\mathrm{P}(n \geqslant 1)$ & $0.8107^{* * *}$ & $0.6938^{* * *}$ & $0.6938^{* * *}$ \\
\hline $\mathrm{P}(n \geqslant 2)$ & 0.3597 & 0.1920 & 0.1941 \\
\hline \multicolumn{4}{|c|}{ Scenario 2.B: Change in Drift } \\
\hline Mean & $2.4251^{* * *}$ & $0.9016^{* * *}$ & $0.7171^{* * *}$ \\
\hline $\mathrm{P}(n \geqslant 1)$ & $0.8606^{* * *}$ & $0.6862^{* * *}$ & $0.5949^{* * *}$ \\
\hline $\mathrm{P}(n \geqslant 2)$ & $0.5320^{* * *}$ & $0.1729^{* * *}$ & 0.1067 \\
\hline \multicolumn{4}{|c|}{ Scenario 3: Change in Discount Rate } \\
\hline Mean & $1.5366^{* * *}$ & $0.9328^{* * *}$ & $0.6283^{* * *}$ \\
\hline $\mathrm{P}(n \geqslant 1)$ & $0.8124^{* * *}$ & $0.7350^{* * *}$ & $0.5739^{* * *}$ \\
\hline $\mathrm{P}(n \geqslant 2)$ & $0.3700^{* * *}$ & $0.1661^{* * *}$ & $0.0510^{* * *}$ \\
\hline \multicolumn{4}{|c|}{ Scenario 4.A: Change in Correlation (Positive) } \\
\hline Mean & $1.0568^{* * *}$ & $0.5076^{* * *}$ & $0.4378^{* * *}$ \\
\hline $\mathrm{P}(n \geqslant 1)$ & $0.7921^{* * *}$ & $0.4990^{* * *}$ & $0.4334^{* * *}$ \\
\hline $\mathrm{P}(n \geqslant 2)$ & $0.1863^{* * *}$ & $0.0085^{* * *}$ & 0.0044 \\
\hline \multicolumn{4}{|c|}{ Scenario 4.B: Change in Correlation (Negative) } \\
\hline Mean & $5.5017^{* * *}$ & $2.6163^{* * *}$ & $2.5173^{* * *}$ \\
\hline $\mathrm{P}(n \geqslant 1)$ & $0.9468^{* * *}$ & $0.9052^{* * *}$ & $0.9060^{* * *}$ \\
\hline $\mathrm{P}(n \geqslant 2)$ & $0.7962^{* * *}$ & $0.6281^{* * *}$ & $0.6150^{* * *}$ \\
\hline
\end{tabular}


TABLE 3.8: Changes in Switching Behaviour Across Three Models When Product 2 Parameters change.

Note 1: This table presents the mean number of switches, the probability of one or more switches taking place, and the probability of two or more switches taking place based on 65000 simulation paths over 200 years. The choice of 65000 and 200 is ad hoc and it should be enough to evaluate the behaviour of agents. I use bootstrap method to generate 10000 random samples with the same size of 65000 and replacement. I use this technique to test if the statistics of the mean number of switches, the probability of one or more switches taking place, and the probability of two or more switches taking place in the projection model vs the cash-cost model and the cash-time model vs the projection model are quantitatively/qualitatively different. The superscripts ${ }^{* *},{ }^{* *}$, and * indicate significance at the $1 \%, 5 \%$, and $10 \%$ level respectively.

\begin{tabular}{llll}
\hline Variable & Cash-Cost Model & Projection Model & Cash-Time Model \\
\hline
\end{tabular}

\section{Baseline Parameters Analysis}

$\begin{array}{llll}\text { Mean } & 1.6049 & 1.0072^{* * *} & 0.9621^{* * *} \\ \mathrm{P}(n \geqslant 1) & 0.8233 & 0.7551^{* * *} & 0.7425^{* * *} \\ \mathrm{P}(n \geqslant 2) & 0.3914 & 0.2011^{* * *} & 0.1804^{* * *}\end{array}$

\section{Scenario 5.A: Change in Cost}

$\begin{array}{llll}\text { Mean } & 0.0959 & 0.0041^{* * *} & 0.0000^{* * *} \\ \mathrm{P}(n \geqslant 1) & 0.0766 & 0.0020^{* * *} & 0.0000^{* * *} \\ \mathrm{P}(n \geqslant 2) & 0.0030 & 0.0001^{* * *} & 0.0000\end{array}$

\section{Scenario 5.B: Change in Time}

$\begin{array}{llll}\text { Mean } & 1.6049 & 0.2352^{* * *} & 0.0178^{* * *} \\ \mathrm{P}(n \geqslant 1) & 0.0593 & 0.0011^{* * *} & 0.0000^{* * *} \\ \mathrm{P}(n \geqslant 2) & 0.0127 & 0.0000^{* * *} & 0.0000\end{array}$

\section{Scenario 5.C: Change in Volatility}

\begin{tabular}{llll} 
Mean & 0.7769 & $0.6487^{* * *}$ & $0.1148^{* * *}$ \\
$\mathrm{P}(n \geqslant 1)$ & 0.2715 & $0.2706^{* * *}$ & $0.0009^{* * *}$ \\
$\mathrm{P}(n \geqslant 2)$ & 0.2414 & $0.1910^{* * *}$ & $0.0004^{* * *}$ \\
\hline
\end{tabular}

Scenario 5.D: Change in Drift

$\begin{array}{llll}\text { Mean } & 0.5780 & 0.5164^{* * *} & 0.0581^{* * *} \\ \mathrm{P}(n \geqslant 1) & 0.0692 & 0.0992^{* * *} & 0.0000^{* * *} \\ \mathrm{P}(n \geqslant 2) & 0.0259 & 0.0134^{* * *} & 0.0000^{* * *}\end{array}$




\subsection{Conclusion}

This chapter develops a framework for analysing the behaviour of agents who have the option to produce different products. Agents change between different products as the net income of competing products changes over time. I set up a partial differential equation model and then solve it with the Alternating Direction Implicit approach. Switching products entails a waiting cost and agents miss their cash flows during this period. The projection and cash-time models provide an optimal solution for options to switch by capturing not only the cash cost but also the time cost.

The cash-cost model is what I see in traditional literature (e.g. Song, Zhao, and Swinton (2011), Livingston, Roberts, and Y. Zhang (2014), Hauer, Luckert, Yemshanov, and Unterschultz (2016), and Hossiso and Ripplinger (2017)) that evaluates a situation where agents pay a cash cost and switch to a new state of activity. The cash-time model enables agents to move to a transition state initially, which allows them to switch back to the original product, if the cash flows change in its favour. In this model, I examine the time effect very carefully and find the exact time cost. Agents in this model must wait for a while in "Transition" state before they become active in a new state of activity. However, agents have the option to abort the switching process and go back to the original product. The projection model is an approximation for the cash-time model where agents have to pay the cash cost but they take into account the opportunity cost and missing the cash flows from existing products. Therefore, agents consider the cash cost and a monetary proxy for the time cost (in terms of foregone profits), for switching to a new state of activity.

My study shows that the cash-cost model that is used throughout the literature overestimates switching frequency. The projection model presents better results compared with the cash-cost model, but underperforms compared with the cash-time model due to approximating the time 
cost. The cash-time model computes the exact time cost and its results are significantly different from the projection model when the time of waiting is substantial. The sensitivity results from the cash-time model are significantly different from the projection model at the $1 \%$ level in all scenarios except in the case when volatility is high. My results indicate that increases in cost, time, volatility, drift, and discount rate have negative impacts on the switch frequency. Correlation has a negative impact on switch frequency if it is positive but a positive impact if it is negative.

The results for this study are important and useful in theory and practice. In theory, I contribute to the literature on valuation, especially the real options approach, by presenting a novel formulation of real options to switch. Researchers can use the results from my study for their analysis or to develop the idea for further exploration. In practice, economics agents, policy makers, analysts, and advisors in industries with similar natures may find my methodology useful. 
118 CHAPTER 3. OPTIMAL ENTERPRISE USE SWITCHING POLICY 


\section{Chapter 4}

\section{Orchards Dataset Analysis}




\subsection{Data Statistics}

The focus of Chapter 4 is to evaluate apple orchards in New Zealand, specifically in Hawke's Bay region. This region is quite well suited to orchards, and growing apples is a lucrative, attractive, and competitive activity. Ministry of Primary Industry reports show that the Hawke's Bay orchards have the highest production and profit for the pipfruit industry in New Zealand (MPI, 2017). In the following, I evaluate orchards and the corresponding statistics.

The apple dataset includes 59197 (orchard-time) observations with 1098 specific identification codes over time period 2010-15. Each identification code is allocated to a specific parcel of land owned by an individual orchardist. For example, orchardist A might have 25 identification codes that are different either in terms of land size or the year of examination. It is worth to note that orchardists might have a portfolio of apple varieties that affects on their decisions to switch when the apple prices change over time. The minimum and maximum land sizes are 0.01 and 22.58 hectare respectively. Apple types are classified into 20 variety groups where similar apple varieties are merged into one variety. ${ }^{1}$ Hence, the apple variety groups shrink from 20 to 14 groups. Table 4.1 presents the amount of total land in hectares that is allocated to each variety group.

Table 4.1 shows that less land is allocated to Nashi, Beauty, and Cox with $0.24 \%, 1.25 \%$, and $2.08 \%$ respectively and more land to apple groups Gala, Braeburn, Jazz, and Fuji with 26.98\%, 17.21\%, 11.12\%, and 10.43\% respectively. This specification is one of the criteria that help to choose the right apple type to consider in the switching model in Chapter 5 model.

Table 4.2 shows how much land is allocated to apple varieties in each

\footnotetext{
${ }^{1}$ For example, High Colour Gala and Gala as Gala, High Colour Braeburn and Braeburn as Braeburn, High Colour Fuji and Fuji as Fuji, and finally High Colour Cripps Pink, Cripps Pink, and Pink Lady as Cripps.
} 
TABLE 4.1: Allocated Land to apple varieties in New Zealand

Note: This table shows the allocated land in hectares to 14 apple varieties in New Zealand. Less land is allocated to Nashi, Beauty, and Cox with $0.24 \%, 1.25 \%$, and $2.08 \%$ respectively and more land to apple groups Gala, Braeburn, and Jazz with $26.98 \%, 17.21 \%$, and $11.12 \%$ respectively. There are 20 apple varieties that shrink to 14 varieties due to combining varieties within the same class.

\begin{tabular}{lcc}
\hline Variety & Total Land & Percentage \\
\hline Nashi & 114.68 & $0.24 \%$ \\
Beauty & 587.18 & $1.25 \%$ \\
Cox & 973.53 & $2.08 \%$ \\
Granny Smiths & 1370.47 & $2.92 \%$ \\
Envy & 1460.48 & $3.12 \%$ \\
Rose & 2030.54 & $4.33 \%$ \\
Pears & 2195.07 & $4.68 \%$ \\
Cripps Pink & 2287.26 & $4.88 \%$ \\
Queen & 2514.35 & $5.36 \%$ \\
Other & 2522.30 & $5.38 \%$ \\
Fuji & 4890.94 & $10.43 \%$ \\
Jazz & 5211.63 & $11.12 \%$ \\
Braeburn & 8068.21 & $17.21 \%$ \\
Gala & 12649.43 & $26.98 \%$ \\
\hline
\end{tabular}


TABLE 4.2: Allocated Land to apple varieties in different Regions in New Zealand

Note: This table shows the amount of allocated land in hectares to apples in different regions in New Zealand. There are 13 regions around the country that are focused on growing apple varieties. Bay of Plenty, Manawatu, and Auckland regions have the lowest land (with $0.06 \%, 0.11 \%$, and $0.16 \%$ respectively) and Hawke's Bay, Nelson, and Otago regions have the largest amount of allocated land (with $61.29 \%, 28.76 \%$, and $4.68 \%$ respectively) for growing apple varieties out of $100 \%$ total land allocated for growing apple in New Zealand.

\begin{tabular}{lcc}
\hline Variety & Total Land & Percentage \\
\hline Bay of Plenty & 29.97 & $0.06 \%$ \\
Manawatu/Wanganui & 51.98 & $0.11 \%$ \\
Auckland & 73.47 & $0.16 \%$ \\
Horowhenua & 111.67 & $0.24 \%$ \\
Marlborough & 118.58 & $0.25 \%$ \\
South Canterbury & 137.56 & $0.29 \%$ \\
Canterbury & 240.96 & $0.51 \%$ \\
Wairarapa & 430.77 & $0.92 \%$ \\
Gisborne & 560.93 & $1.20 \%$ \\
Waikato & 714.64 & $1.52 \%$ \\
Otago & 2194.81 & $4.68 \%$ \\
Nelson & 13482.24 & $28.76 \%$ \\
Hawke's Bay & 28728.49 & $61.29 \%$ \\
\hline
\end{tabular}


region. Bay of Plenty, Manawatu, and Auckland regions have the lowest land (with $0.06 \%, 0.11 \%$, and $0.16 \%$ respectively) and Hawke's Bay, Nelson, and Otago regions have the largest amount of allocated land (with $61.29 \%, 28.76 \%$, and $4.68 \%$ respectively) for growing apple varieties out of $100 \%$ total land allocated for growing apple in New Zealand. The apple dataset also gives information about the year that trees are planted. This is important, since it allows us to measure the apple trees' age.

The number of observations in the dataset decreases from 59197 (1098 IDs) to 50504 (1027 IDs) observations after filtering all regions by deleting observations with zero land area, missing year planted, and finally merging similar apple varieties into one variety. The orchardists with blocks of land plant trees with different apple types. Some orchardists might split a large block of land to small blocks or combine small blocks to a large block of land. In this regard, it is hard to recognise if this is exactly the same block of land that is under examination. To overcome this difficulty, I create a new identification code for orchards by combining parcel identification code, planting year, apple type, and the area of land that is allocated each year to track orchardists' activity through time. Therefore the new identification code is a parcel-time code and it becomes clearer which identification code is traceable over time by controlling key factors over time. The new identification code breaks down the total 50504 (1027 IDs) observations into 38597 (11334 IDs) traceable observations and 11907 (4902 IDs) non-traceable observations. The dataset with traceable observations provides a robust examination of orchardists' behaviour. I create a dummy variable for apple varieties to examine how the apple type changes for each specific orchardist in traceable dataset. If the apple type for the new identification code remains the same, the dummy variable equals zero, and otherwise it equals one. Hence, there are, 1,950 (440 IDs) observations that switch from one type of apple to another. Table 4.3 maps all the switches to discover the switch direction and orchardists' preferences towards each apple variety. Results show that there are 420 
TABLE 4.3: The Number of Switches Among Apple Varieties in All Regions.

Note: This table shows the number of switches among apple varieties in all regions in New Zealand. Orchardists switch from one type of apple to another since the profitability of an apple variety changes over time. The total number of switches From and To each apple variety are reported in the last column and row respectively.

\begin{tabular}{|c|c|c|c|c|c|c|c|c|c|c|c|c|c|c|c|c|}
\hline \multirow{2}{*}{ 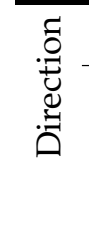 } & & \multicolumn{15}{|c|}{ To } \\
\hline & 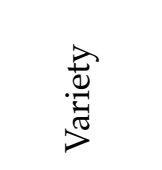 & 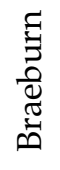 & $\begin{array}{l}\tilde{\Xi} \\
\stackrel{\Xi}{2} \\
a\end{array}$ & $\frac{\pi}{\pi}$ & $\begin{array}{l}: \overline{\sqrt{3}} \\
\text { 竞 }\end{array}$ & 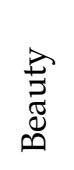 & $\sum_{\vec{y}}^{\vec{c}}$ & 足 & 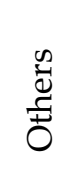 & $\underset{\underset{N}{N}}{\mathbb{N}}$ & 苂 & $\begin{array}{l}\tilde{D} \\
\stackrel{0}{0} \\
\stackrel{1}{1}\end{array}$ & ố & 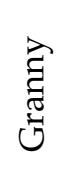 & $\begin{array}{l}\ddot{Z} \\
\text { D } \\
\tilde{Z}\end{array}$ & 푬 \\
\hline \multirow{15}{*}{ 总 } & Braeburn & 0 & 32 & 14 & 18 & 0 & 1 & 3 & 5 & 3 & 3 & 4 & 0 & 2 & 0 & 85 \\
\hline & Queen & 1 & 0 & 3 & 0 & 0 & 0 & 2 & 1 & 0 & 2 & 3 & 0 & 0 & 0 & 12 \\
\hline & Gala & 9 & 12 & 0 & 6 & 0 & 6 & 4 & 3 & 9 & 0 & 6 & 0 & 3 & 0 & 58 \\
\hline & Fuji & 5 & 27 & 21 & 0 & 0 & 11 & 3 & 4 & 4 & 0 & 4 & 1 & 1 & 0 & 81 \\
\hline & Beauty & 0 & 3 & 4 & 2 & 0 & 4 & 0 & 1 & 0 & 0 & 1 & 0 & 0 & 0 & 15 \\
\hline & Envy & 0 & 0 & 4 & 1 & 0 & 0 & 1 & 0 & 2 & 0 & 0 & 0 & 0 & 0 & 8 \\
\hline & Cripps & 1 & 6 & 3 & 3 & 0 & 1 & 0 & 1 & 2 & 0 & 0 & 0 & 0 & 0 & 17 \\
\hline & Other & 6 & 7 & 27 & 7 & 0 & 3 & 3 & 0 & 0 & 6 & 2 & 1 & 1 & 1 & 64 \\
\hline & Jazz & 2 & 7 & 10 & 9 & 0 & 4 & 1 & 1 & 0 & 1 & 0 & 0 & 1 & 0 & 36 \\
\hline & Pears & 0 & 0 & 1 & 0 & 0 & 0 & 0 & 3 & 0 & 0 & 2 & 0 & 0 & 0 & 6 \\
\hline & Rose & 1 & 2 & 12 & 0 & 0 & 0 & 0 & 2 & 0 & 2 & 0 & 0 & 0 & 0 & 19 \\
\hline & Cox & 4 & 3 & 1 & 2 & 0 & 2 & 0 & 1 & 0 & 0 & 1 & 0 & 0 & 0 & 14 \\
\hline & Granny & 0 & 0 & 4 & 0 & 0 & 0 & 0 & 0 & 0 & 0 & 1 & 0 & 0 & 0 & 5 \\
\hline & Nashi & 0 & 0 & 0 & 0 & 0 & 0 & 0 & 0 & 0 & 0 & 0 & 0 & 0 & 0 & 0 \\
\hline & Total & 29 & 99 & 104 & 48 & 0 & 32 & 17 & 22 & 20 & 14 & 24 & 2 & 8 & 1 & $42 C$ \\
\hline
\end{tabular}

IDs that switch once from one type of apple to another and 20 IDs that switch multiple times. Table 4.3 shows that in total, less orchardists switch from Nashi, Granny Smiths, and Pears with 0, 5, and 6 number of switches respectively and more from Braeburn, Fuji, and Other with 85, 81, and 64 number of switches respectively. In contrast, less orchardists switch to Beauty, Nashi, and Cox with 0,1 , and 2 number of switches respectively and more to Gala, Queen, and Fuji with 104, 99, and 48 number 
TABLE 4.4: Allocated Land Based on Switches in All Regions

Note: This table shows the amount of allocated land in hectares and the number of switches across different regions in New Zealand. Switches occur in 8 different regions: Hawke's Bay, Nelson, and Otago with 359.00,71, and 15 number of switches respectively. The amount of allocated land and the number of switches follow the same pattern, the higher allocated land, the higher number of switches.

Note: This table is different from Table 4.2, because it includes only the land size with switches.

\begin{tabular}{lcccc}
\hline \multicolumn{5}{c}{ Allocated Land and Switches Across Regions } \\
\hline \multicolumn{1}{c}{ Region } & Total Land & Percent & Switch & Percent \\
\hline Marlborough & 0.92 & 0.06 & 1.00 & 0.22 \\
Horowhenua & 2.76 & 0.18 & 1.00 & 0.22 \\
Waikato & 4.67 & 0.30 & 2.00 & 0.43 \\
Wairarapa & 17.86 & 1.14 & 8.00 & 1.73 \\
Gisborne & 33.89 & 2.16 & 5.00 & 1.08 \\
Otago & 44.74 & 2.85 & 15.00 & 3.25 \\
Nelson & 391.44 & 24.95 & 71.00 & 15.37 \\
Hawke's Bay & 1072.43 & 68.37 & 359.00 & 77.72 \\
\hline
\end{tabular}

of switches respectively. Among all combinations, results show that orchardists switch mainly from Braeburn to Queen, from Fuji to Queen, and from Other to Gala with 32, 27, and 27 number of switches.

I examine the number of switches according to the regions and corresponding allocated land. Table 4.4 shows that approximately $70 \%$ of allocated land and $80 \%$ of the switches belongs to Hawke's Bay. Nelson with approximately $25 \%$ of allocated land and $15 \%$ number of switches is the second important region. The rest of regions may be ignored given the allocated land along with the low number of switches. The number of switches in Table 4.4 includes both single and multiple switches.

The dataset evaluation emphasizes the importance of Hawke's Bay region to be the target regions due to having the highest amount of allocated land to apple varieties and higher number of switches. The number 
TABLE 4.5: Allocated Land to apple varieties in Hawke's Bay

Note: This table shows the allocated land in hectares to apple in Hawke's Bay. Nashi, Cox, and Beauty with $0.02 \%, 0.22 \%$, and $1.60 \%$ respectively, have the least allocated land, and Gala, Braeburns, and Fuji with $30.21 \%, 16.32 \%$, and $13.16 \%$ respectively, have the largest amount of allocated land to apple varieties.

\begin{tabular}{clcl}
\hline \multicolumn{4}{l}{ Allocated Land to Apple Varieties } \\
\hline Obs & Variety Group & Total Land & Percent \\
\hline 1 & Nashi & 5.24 & 0.02 \\
2 & Cox & 63.91 & 0.22 \\
3 & Beauty & 460.36 & 1.60 \\
4 & Envy & 614.57 & 2.14 \\
5 & Pears & 730.55 & 2.54 \\
6 & Granny Smiths & 978.38 & 3.41 \\
7 & Other & 1045.35 & 3.64 \\
8 & Cripps Pink & 1504.04 & 5.24 \\
9 & Rose & 1773.75 & 6.17 \\
10 & Jazz & 2121.08 & 7.38 \\
11 & Queen & 2285.23 & 7.95 \\
12 & Fuji & 3780.25 & 13.16 \\
13 & Braeburn & 4687.77 & 16.32 \\
14 & Gala & 8678.05 & 30.21 \\
\hline
\end{tabular}

of observations for Hawke's Bay region is 34237 (640 IDs) after implementing the same filtering process that I had for all regions. Table 4.5 presents the amount of allocated land to each apple variety in Hawke's Bay region.

Table 4.5 shows that Nashi, Cox, and Beauty with $0.02 \%, 0.22 \%$, and $1.60 \%$ respectively, have the least allocated land, and Gala, Braeburns, and Fuji with $30.21 \%, 16.32 \%$, and $13.16 \%$ respectively, have the largest amount of allocated land among competitive apple varieties. I follow the same filtering process to calculate the number of switches whose land parcels are 
traceable and map them among apple varieties. The first row and column in Table 4.6 show the switching direction and the last row and column show the total number of switches to and from respectively. Results in Table 4.6 show that there are 326 single switches from one type of apple to another and 16 multiple switches from one type of apple to another and then to another apple type that raise the total number of switches to 342.

Table 4.6 shows that orchardists in Hawke's Bay, switch from one type of apple to another. The total number of switches from and to each apple variety are reported in the last column and row respectively. In terms of total switches, less orchardists switch from Cox, Pears, and Granny Smiths with 3, 3, and 4 switches respectively and more from Braeburn, Fuji, and Gala with 81, 59, and 47 switches respectively. In contrast, less orchardists switch to Beauty, Nashi, and Granny Smiths with 0, 1, and 5 number of switches respectively and more to Queen, Gala, and Fuji with 94, 79, and 41 switches respectively. Orchardists switch mainly from Braeburn to Queen, from Fuji to Queen, and from Other to Gala with 32, 26, and 17 switches respectively. The switching pattern among apple varieties in Hawke's Bay is the same as the switching pattern in all regions in Table 4.3. This implies that Hawke's Bay are representative of the industry more generally.

Table 4.7 reports the price path of apple varieties from 2007-16. There is a longer historical apple price series compared with the orchardists' dataset. ${ }^{1}$ Apple prices are reported in dollar per Tray Carton Equivalent (TCE) and includes 12 types of apples. The missing types (Envy and Nashi) are deleted due to having missing values. ${ }^{2}$

\footnotetext{
1 The table of apple prices is good to find the parameters and the more the better. Unfortunately we have a short historical data. To evaluate the behaviour of orchardist we have only the data for 2010-2015.

${ }^{2}$ A TCE is defined as $18.6 \mathrm{~kg}$ packed weight and equates to $18.0 \mathrm{~kg}$ sale weight.
} 
TABLE 4.6: The Number of Switches Among Apple Varieties in Hawke's Bay

Note: This table shows the number of switches among apple varieties in Hawke's Bay. Orchardists switch from one type of apple to another since the profitability of an apple variety changes over time. The total number of switches From and To each apple variety are reported in the last column and row respectively. In terms of total switches, less orchardists switch from Cox, Pears, and Granny Smiths with 3, 3, and 4 number of switches respectively and more from Braeburn, Fuji, and Gala with 81, 59, and 47 number of switches respectively. In contrast, less orchardists switch to Beauty, Nashi, and Granny Smiths with 0, 1, and 5 number of switches respectively and more to Queen, Gala, and Fuji with 94, 79, and 41 number of switches respectively. Orchardists switch mainly from Braeburn to Queen, from Fuji to Queen, and from Other to Gala with 32, 26, and 17 number of switches respectively.

\begin{tabular}{|c|c|c|c|c|c|c|c|c|c|c|c|c|c|c|c|}
\hline \multirow{2}{*}{ 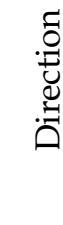 } & \multicolumn{15}{|c|}{ To } \\
\hline & 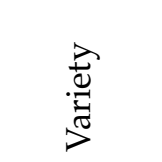 & 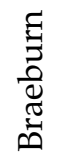 & 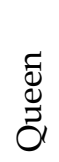 & $\frac{\tilde{\sigma}}{\tilde{J}}$ & :责 & $\begin{array}{l}\underset{\Xi}{\vec{J}} \\
\stackrel{D}{D}\end{array}$ & $\sum_{\text {空 }}^{\overrightarrow{1}}$ & 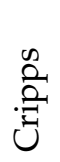 & $\begin{array}{l}\frac{0}{\mathscr{E}} \\
\frac{ \pm}{0}\end{array}$ & $\begin{array}{l}\text { N } \\
\text { త్ }\end{array}$ & 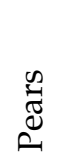 & $\underset{\mathscr{D}}{\mathscr{D}}$ & 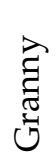 & $\begin{array}{l}\vec{z} \\
\text { D } \\
\tilde{Z}\end{array}$ & $\underset{0}{\tilde{0}}$ \\
\hline \multirow{14}{*}{ 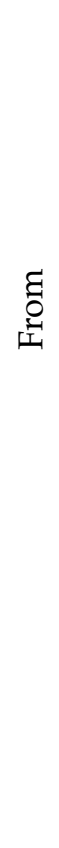 } & Braeburn & 0 & 32 & 14 & 18 & 0 & 1 & 2 & 4 & 3 & 1 & 5 & 1 & 0 & 81 \\
\hline & Queen & 1 & 0 & 3 & 0 & 0 & 0 & 2 & 1 & 0 & 2 & 3 & 0 & 0 & 12 \\
\hline & Gala & 8 & 11 & 0 & 5 & 0 & 5 & 4 & 1 & 6 & 0 & 5 & 2 & 0 & 47 \\
\hline & Fuji & 3 & 26 & 16 & 0 & 0 & 2 & 2 & 3 & 4 & 0 & 2 & 1 & 0 & 59 \\
\hline & Beauty & 0 & 3 & 3 & 2 & 0 & 4 & 0 & 1 & 0 & 0 & 0 & 0 & 0 & 13 \\
\hline & Envy & 0 & 0 & 2 & 1 & 0 & 0 & 1 & 0 & 1 & 0 & 0 & 0 & 0 & 5 \\
\hline & Cripps & 1 & 7 & 3 & 3 & 0 & 0 & 0 & 0 & 2 & 0 & 0 & 0 & 0 & 16 \\
\hline & Other & 0 & 6 & 17 & 3 & 0 & 2 & 2 & 0 & 0 & 4 & 2 & 0 & 1 & 37 \\
\hline & Jazz & 1 & 7 & 8 & 8 & 0 & 4 & 0 & 0 & 0 & 1 & 0 & 1 & 0 & 30 \\
\hline & Pears & 0 & 0 & 0 & 0 & 0 & 0 & 0 & 1 & 0 & 0 & 2 & 0 & 0 & 3 \\
\hline & Rose & 1 & 2 & 10 & 0 & 0 & 0 & 0 & 2 & 0 & 1 & 0 & 0 & 0 & 16 \\
\hline & Cox & 2 & 0 & 0 & 1 & 0 & 0 & 0 & 0 & 0 & 0 & 0 & 0 & 0 & 3 \\
\hline & Granny & 0 & 0 & 3 & 0 & 0 & 0 & 0 & 0 & 0 & 0 & 1 & 0 & 0 & 4 \\
\hline & Total & 17 & 94 & 79 & 41 & 0 & 18 & 13 & 13 & 16 & 9 & 20 & 5 & 1 & 326 \\
\hline
\end{tabular}




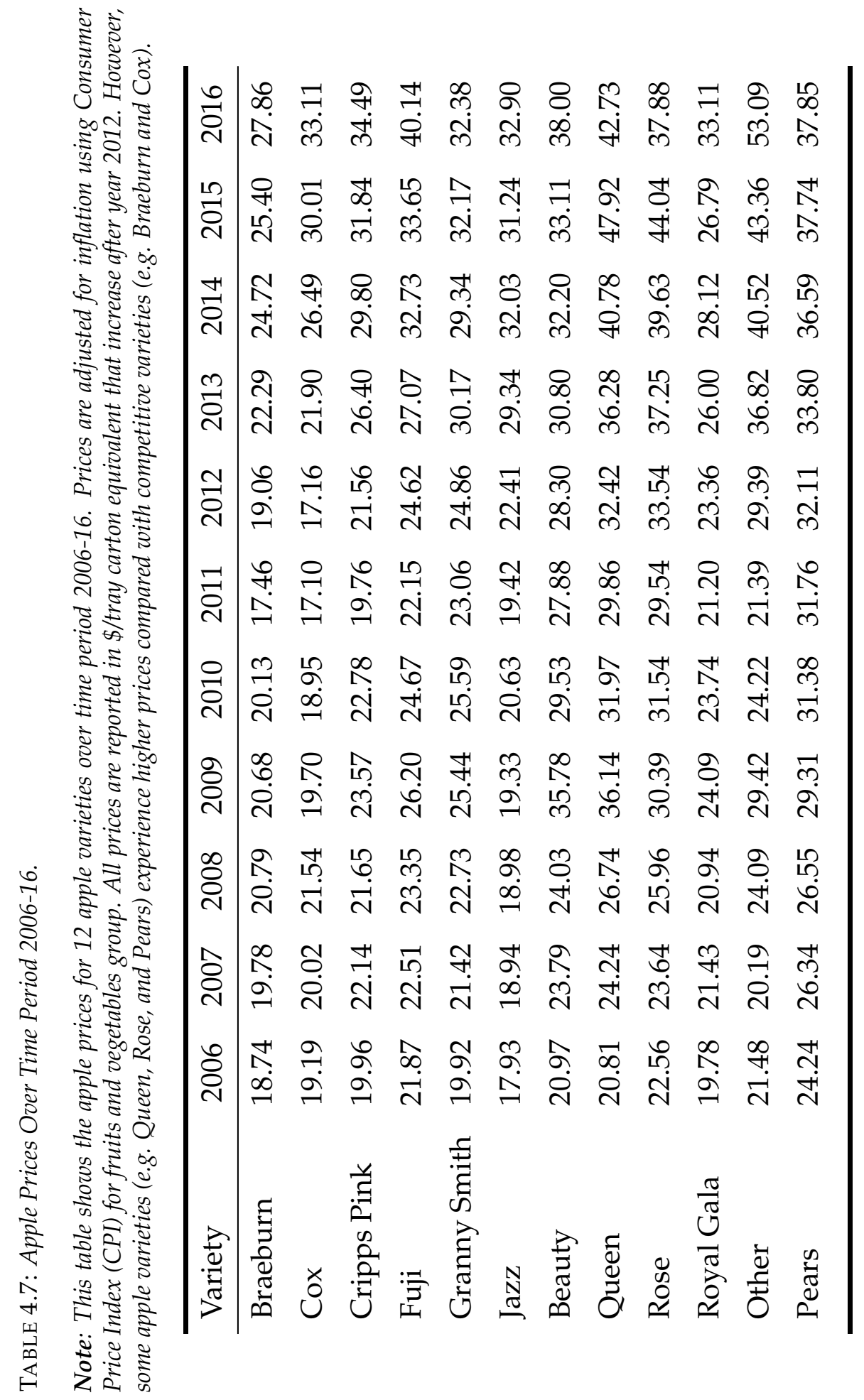


Table 4.7 shows that the apple prices tend to increase over time. Queen, Rose, and Pears experience the highest prices whereas Braeburn and Cox experience the lowest prices among competitive apple varieties. Figure 4.1 displays the apple prices path from 2006 to 2016.

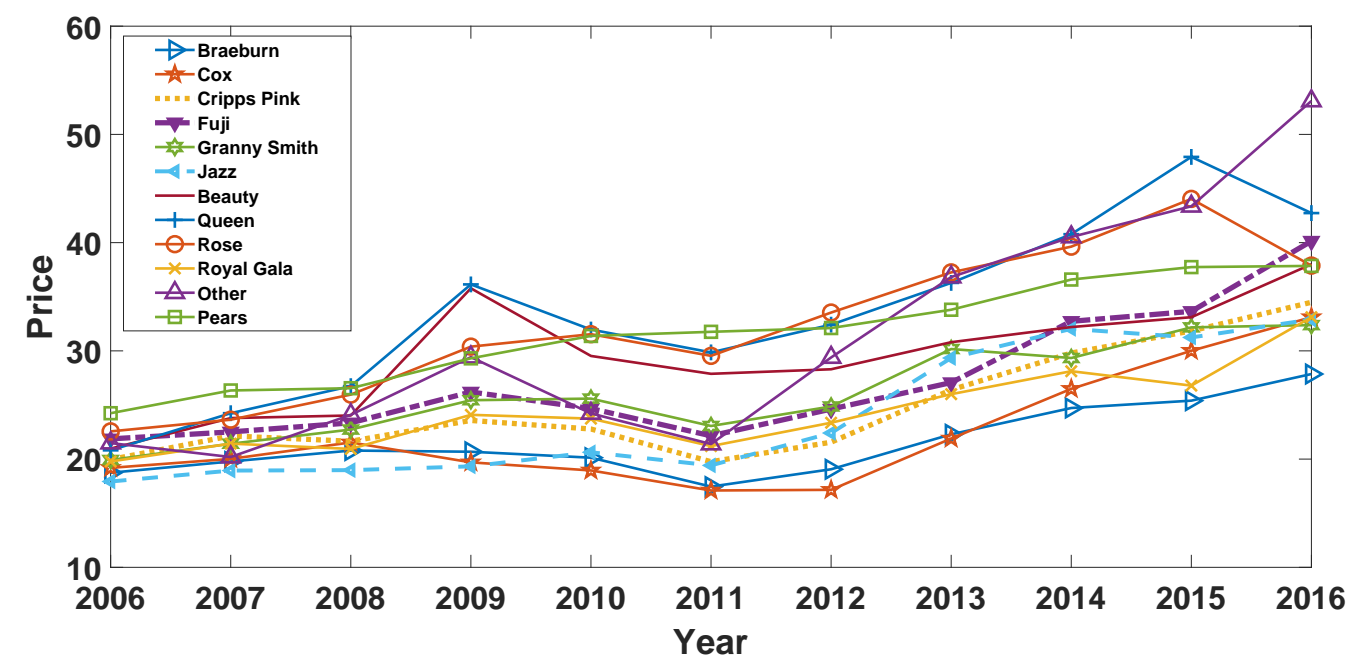

FIgURE 4.1: Apple Prices Path Over Time Period 2006-16.

Note: This figure shows the apple prices path over time period 2006-16. The horizontal axis shows the years and the vertical axis shows the apple prices, which are adjusted for inflation by CPI. Queen, Rose, and Pears experience the highest prices whereas Braeburn and Cox experience the lowest prices among competitive apple varieties.

Among all apple varieties, Braeburn and Queen are two candidates to be examined. Figure 4.2 shows the ratio of Braeburn price to Queen price over time period 2006-16. The ratio of these two apple prices has been volatile with decreasing trend over this time period. In the following, I will describe how Braeburn and Queen apple types are the best candidates for the set up model.

Apple types Queen, Beauty, and Other are more volatile over time whereas Jazz, Pears, and Braeburn are less volatile. According to the Ministry of Primary Industry (MPI) Model for Hawkes Bay, orchardists pick 3104 tray carton apples in each hectare, which gives a reference to scale the apple prices per hectare. ${ }^{1}$ To avoid any biases and random choice of ap-

\footnotetext{
${ }^{1}$ MPI reports two pipfruit models: Hawkes Bay pipfruit model and Nelson pipfruit
} 


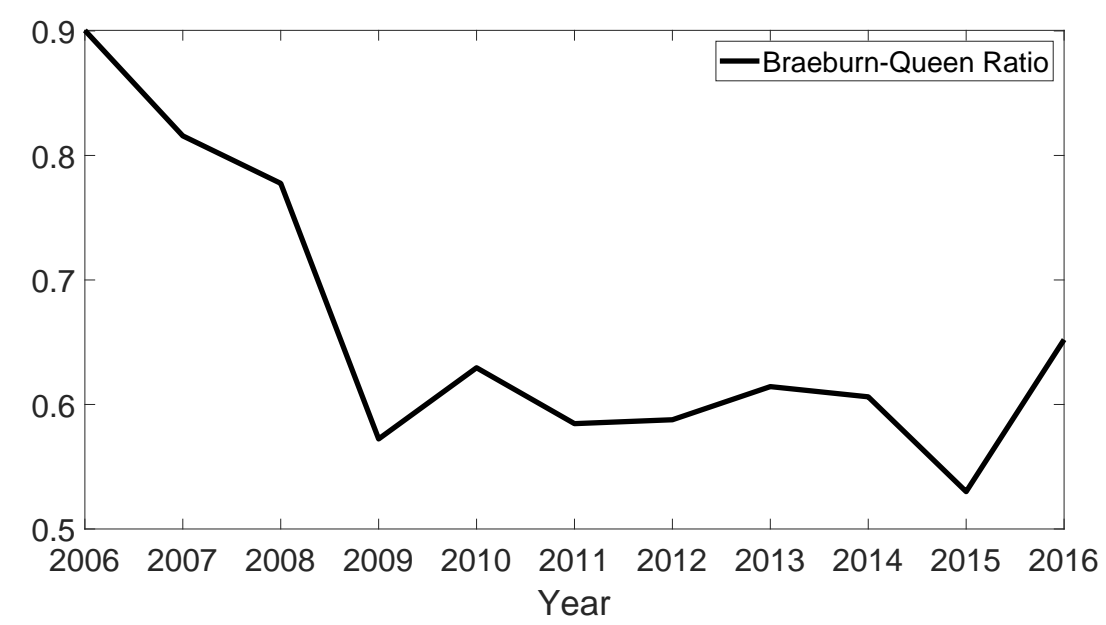

FIGURE 4.2: Ratio of Braeburn Price to Queen Price Over Time Period 2006-16.

Note: This figure shows the ratio of Braeburn apple price to Queen price over time period 2006-16. The horizontal axis shows the years and the vertical axis shows the apple prices ratio.

ple variety for my model, I exploit a classification technique to narrow the choice from 15 different apple varieties into two variety groups. Hence, I use the Principal Component Analysis and K-means clustering as two unsupervised clustering methods. The Principal Component Analysis tries to find the trends and patterns in the dataset by reducing dimensions into a limited number of principal components. K-means (like Principal Component Analysis) clusters by putting all observations into K cluster by optimising the mean between each cluster. The apple prices are based on dollar per hectare $(\$ /$ ha), which is the product of prices $(\$ /$ tce $)$ with 3104 trays for each hectare. Also, I normalise the apple prices by subtracting their mean and dividing by their standard deviation to avoid biases in clustering. The clustering technique with the aim of having two clusters specifies that Braeburn, Cripps Pink, Granny Smiths, Jazz, Queen, Rose, and pears belong to Cluster-1 whereas Fuji, Beauty, Royal Gala, and other belong to Cluster-2. My model is set up for a switching option with two

model. These two regions are the main pipfruit growing in New Zealand (see Table 4.4). Each model is based on the valuation of production and profitability for 40 hectares planted orchards in each region. The average $3104 \mathrm{TCE} / \mathrm{ha}$ is for any type of apple. 
products and requires estimation of drifts, volatilities, and correlation parameters. I estimate drift and volatility of log revenues under the assumption of a GBM as follows:

$$
\begin{aligned}
\mu_{i} & =\frac{\widehat{\alpha}_{i}}{\Delta t}+\frac{1}{2}\left(\frac{\widehat{\phi}_{i}}{\sqrt{\Delta t}}\right)^{2} \\
\sigma_{i} & =\frac{\widehat{\phi}_{i}}{\sqrt{\Delta t}}
\end{aligned}
$$

where $\mu_{i}$ is drift, $\widehat{\alpha_{i}}$ is the mean of changes in log revenues, $\Delta t$ is time increment, and $\widehat{\phi}_{i}$ is the standard deviation of changes in log revenues. The first method is to take the average of apple prices in each cluster that accounts for the corresponding apple types 1 and 2. Using the average log prices in Table 4.8 and plugging them into equation (4.1) gives drifts of $5 \%$ and $6 \%$, volatilities of $7 \%$ and $11 \%$ for apple clusters 1 and 2 respectively, and 0.56 correlation between the two apple clusters. The result of considering the average log prices in each cluster is that the high volatility apple types get affected by less volatile types. Hence, the model would be expected to reveal less switching when using drift, volatility, and correlation parameters. 


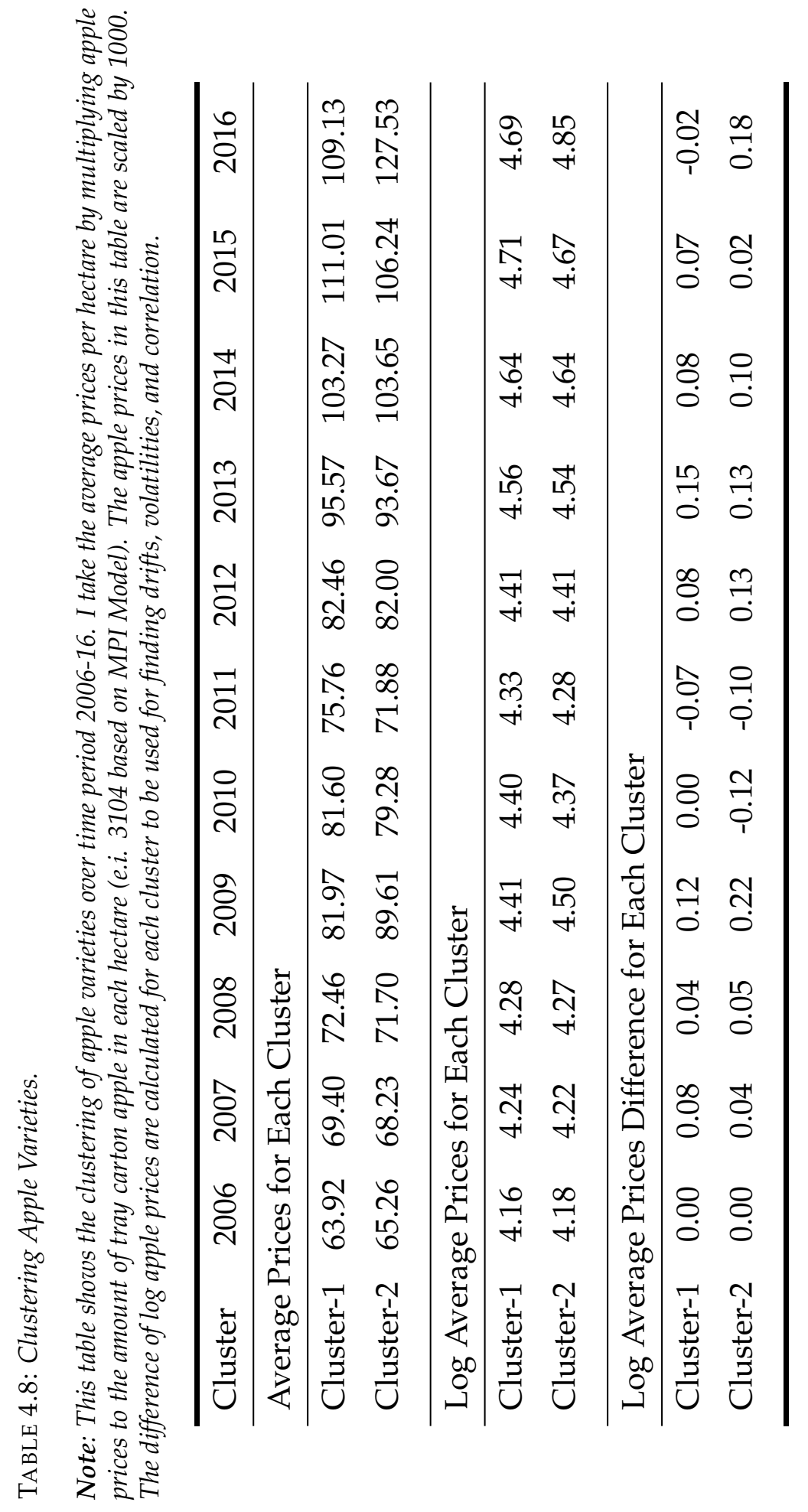


In the second approach, I use the maximum price among all apple prices in cluster- 1 as the first price series and the minimum price among all apple prices in cluster- 2 as the second price series over time. Hence, by having the two apple price series I calculate the drift, volatility, and correlation variables of model. Similarly, I find the variables by using the minimum price among apple prices in cluster-1 and maximum price among all apple prices in cluster-2 over time. All the apple prices are in terms of $\log$ price. In this approach, the results refer to a group of apple types instead of a particular type. An alternative approach is to select a particular apple type with the highest price versus a particular apple type with the lowest price. This approach removes the ambiguity of having a group of apple type as a whole. Queen, Gala (with high prices), and Braeburn (with low prices) are good candidates for this approach. Queen has 8\% drift and 13\% volatility, Gala has 6\% drift and 10\% volatility, and finally Braeburn has $4 \%$ drift and $8 \%$ volatility. The correlation for the QueenBraeburn pair is 0.26 , whereas the correlation for the Gala-Braeburn pair is 0.68. Queen and Braeburn with different volatilities are better candidates than the Gala and Braeburn pair with the same volatilities. Hence, the focus of study narrows down into two types of apple with more clarity. In the next chapter, I set up my model for two options to switch and this simplification helps to select only two suitable candidates for examination.

The final test on the apple dataset is to look at the age of the apple trees. In this regard, I select the traceable dataset in all regions and check if the planted year changes with the change in apple variety. If the planted year changes with the change in apple variety, it implies that orchardists replace the existing apple tree with another variety. In contrast, if the planted year remains the same but the type of apple changes, it implies grafting another apple type on the existing rootstock. There are 1330 (238 IDs) observations that account for replacing and 620 (103 IDs) observations that account for grafting. Table 4.9 shows how orchardists change the apple type either by uprooting and planting young apple trees, or by grafting 
TABLE 4.9: Age Groups of Switches in All Region.

This table presents the orchardists who switch by uprooting and planting new apple trees or by grafting on the existing rootstocks. The number of switches and the corresponding percentage are reported in different columns. The strategy of switching is categorised according to the age of apple trees. The uproot-plant strategy is more likely in age categories 20-25 and 8-20 years with $81.45 \%$ and $75.41 \%$ respectively. In contrast, the grafting strategy is more likely in age categories $0-8,8-20$, and $20-25$ years with $58.14 \%, 24.59 \%$, and $18.55 \%$ respectively.

\begin{tabular}{lcccc}
\hline \multirow{2}{*}{ Age Group } & \multicolumn{2}{c}{ Number of Observations } & \multicolumn{2}{c}{ Percentage of Observations } \\
\cline { 2 - 5 } & Uproot-Plant & Graft & Uproot-Plant & Graft \\
\hline $0-8$ & 36 & 50 & $41.86 \%$ & $58.14 \%$ \\
$8-20$ & 92 & 30 & $75.41 \%$ & $24.59 \%$ \\
$20-25$ & 101 & 23 & $81.45 \%$ & $18.55 \%$ \\
$>30$ & 9 & 0 & $100.00 \%$ & $00.00 \%$ \\
Total & 238 & 103 & $69.79 \%$ & $30.21 \%$ \\
\hline
\end{tabular}

on existing trees as a function of tree's age, with old trees getting uprooted. The number of switches and the corresponding percentage are reported in different columns. The strategy of switching is categorised according to the age of apple trees. The age is measured by finding the difference between the current year and planted year of apple trees. The uproot-plant strategy is more likely in age categories $20-25$ and 8-20 years with $81.45 \%$ and $75.41 \%$ respectively. In contrast, the grafting strategy is more likely in age categories $0-8,8-20$, and $20-25$ years with $58.14 \%, 24.59 \%$, and $18.55 \%$ respectively.

As a follow up, I examine if the uproot-plant and the graft strategies are statistically significantly different according to the apple trees' age. Hence, I implement a two sample t-test with unequal variance to see if the uproot-plant and the graft samples (two switching strategies) are significantly different when the tree's age changes. My intuition is that the uproot-plant strategy is employed for old apple trees, whereas the graft strategy is ideal for young trees. Table 4.10 shows the results of the t-test with a null hypothesis of having the same mean between two samples 
TABLE 4.10: $t$-test Results for Switches Based on Trees' Age.

This table presents the t-test between the apple trees' age in two samples: uproot-plant and graft. There are 238 orchardists who employ the uproot-plant strategy, where the trees' age have the mean 19.1218 with 7.5578 standard deviation. In contrast, there are 103 orchardists who employ the graft strategy, where the trees' age have the mean 12.2788 with 8.6546 standard deviation. I use a t-test with unequal variance to examine my null hypothesis, whether the difference in means is 0 . According to the $t$-test results, the t-value is greater than the critical value and I reject the null hypothesis. In other words, these two switching strategies are employed at different ages and each strategy is ideal for a specific tree's age. Note that apple trees have ages from 1 year old to 33 years old and more.

\begin{tabular}{lcc}
\hline \multicolumn{1}{c}{ Parameters } & Uproot-Plant Age & Graft Age \\
\hline Observations & 238 & 103 \\
Ages Mean & 19.12 & 12.28 \\
Ages Variance & 57.12 & 74.90 \\
Ages Standard Deviation & 7.56 & 8.66 \\
\hline Degree of freedom & 175 & \\
t-Statistics & 6.98 & \\
$\mathrm{P}(\mathrm{T}<=\mathrm{t})$ one-tail & 0.00 & \\
$\mathrm{t}-$ Critical one-tail & 1.65 & \\
$\mathrm{P}(\mathrm{T}<=\mathrm{t})$ two-tail & 0.00 & \\
$\mathrm{t}-$ Critical two-tail & 1.97 & \\
\hline
\end{tabular}


with unequal variance. I reject the null hypothesis in favour of the alternative that the uproot-plant sample mean is significantly different from the graft sample mean at the $1 \%$ level. I evaluate the uproot-plant strategy versus the graft strategy in my model in chapter 5 and compare the results with what orchardists adopt in practice.

Another key parameter is the discount rate, which is the main foundation of valuation in finance. In the real options literature, there is a range of different approaches of adopting the discount rate: (1) to assume an ad hoc discount rate and then changing it in a sensitivity analysis framework (see Majd and Pindyck (1987), Song, Zhao, and Swinton (2011), Schmit, Luo, and Conrad (2011), Shah and Ando (2016), M. Zhang, P. Zhou, and D. Zhou (2016), Hossiso and Ripplinger (2017)), (2) to use a proxy such as central bank discount rate with/without modification (see Odeninga, Muhoff, and Balmannb (2005), Ihli, Maart-Noelck, and Musshoff (2013)), (3) to approximate a long term historical average bond returns as a measure of opportunity cost, (4) and finally to use a required return either capital asset pricing model (CAPM) or dividend growth model (DGM) (see Purvis, Boggess, Moss, and Holt (1995), and Hauer, Luckert, Yemshanov, and Unterschultz (2016)). The main driving forces to specify the discount rate are the assumptions of risk preference and complete markets (Bulan, Mayer, and Somerville, 2009). The two well-known models for this purpose are the DGM and the CAPM. The DGM states that the required return of a security is the sum of its dividend yield and capital gain yield, $R=\frac{D_{1}}{P_{0}}+g$. Table 4.11 presents the parameters for estimating the discount rate. I estimate $D_{1}$ as the price of renting land (\$/ha), $P_{0}$ as the average 10-year price of land (\$/ha), and finally $g$ is the average drift across both apple types (Gala and Braeburn). Note that leasing land for livestock farming is much easier compared to agriculture, due to the fast and easy switch process. A land for running an orchard needs at least 5-6 years time to become productive and it would not be viable to lease empty land for a shorter period of time. So, the contracts for this purpose do not follow an infor- 
TABLE 4.11: The DGM Approach to Estimate Discount Rate.

This table presents the parameters to estimate discount rate based on the DGM model for Chapter 5. Here, $D_{1}$ is the price of renting land, $P_{0}$ is the average 10-year price of land $(\$ / \mathrm{ha})$, and finally $g$ is the average drift of both apple types.

\begin{tabular}{lcc}
\hline \multicolumn{1}{c}{ Parameters } & Symbol & Value \\
\hline 10-years Average sales $(\$ /$ ha) & $D_{1}$ & 167600 \\
Horticulture Rent $(\$ /$ ha) & $P_{0}$ & 1114 \\
Average Drifts & $g$ & 0.06 \\
\hline
\end{tabular}

mal procedure of negotiation but a more formal one with tight rules and regulations through agencies to avoid any conflict. To this end, I estimates $6.87 \%$ discount rate according to the DGM.

The second approach, the CAPM model, states that the required return is the summation of the risk free rate and the risk premium from investing in the market as follows:

$$
\begin{aligned}
& r_{i}=r_{f}+\beta\left(r_{m}-r_{f}\right) \\
& \beta=\frac{\rho_{i, m} \sigma_{i} \sigma_{m}}{\sigma_{m}^{2}},
\end{aligned}
$$

where $r_{i}$ is the required return on apple $i, r_{f}$ is the risk free rate, $r_{m}$ is the expected return on market, $\beta$ is the market risk, $\rho_{i, m}$ is the correlation between apple $i$ 's returns and the market return, $\sigma_{i}$ is apple $i$ 's volatility, and $\sigma_{m}$ is the market volatility. Table 4.12 shows the estimated parameter to use for the CAPM model. I use numbers from Frijns and Tourani-Rad (2016) to measure the market risk $\left(\sigma_{m}\right)$ and risk premium $\left(r_{m}-r_{f}\right)$ in the New Zealand market. I also measure the risk free rate $\left(r_{f}\right)$ from reserve bank treasury bills and the correlation through the time series of stock market return and apple revenues $\left(\rho_{i, m}\right)$. I estimate $\alpha 1.99 \%$ discount rate using the CAPM model, which is lower than the first method. However, the discount rate from DGM approach fits better in my model due to being 
TABLE 4.12: The CAPM Approach to Estimate Discount Rate.

This table presents the parameters to estimate discount rate based on the CAPM model for Chapter 5. I use numbers from Frijns and Tourani-Rad (2016) to measure the market risk $\left(\sigma_{m}\right)$ and risk premium $\left(r_{m}-r_{f}\right)$ in the New Zealand market. I also measure the risk free rate $\left(r_{f}\right)$ from reserve bank treasury bills and the correlation through the time series of stock market return and apple revenues $\left(\rho_{m, i}\right)$.

\begin{tabular}{lcc}
\hline \multicolumn{1}{c}{ Parameters } & Symbol & Value \\
\hline Risk Premium (Geometric Mean- Frijns and Tourani-Rad (2016)) & $r_{m}-r_{f}$ & 0.03 \\
Correlation (Stock, Avg Crop Prices) & $\rho_{m, i}$ & -0.22 \\
Risk Free (Reserve Bank) & $r_{f}$ & 0.02 \\
Average Crops Variances & $\sigma_{i}$ & 0.11 \\
Market Volatility (Frijns and Tourani-Rad (2016)) & $\sigma_{m}$ & 0.20 \\
Estimated Volatility in Use & $\sigma_{i} / \sigma_{m}$ & 0.20 \\
\hline
\end{tabular}

more compatible with main parameters: drift, volatility, and correlation. 


\section{Chapter 5}

\section{Building A Complex Switching Option Model And Its Application In Horticulture}




\subsection{Introduction}

There has been a considerable increase in the number of papers on real options over the past decades. Hence, my study stands on the shoulder of large body of literature. ${ }^{1}$ Reviewing the literature indicates that this theory is analysed well. However, there is a gap in the literature to see whether practitioners adopt real options valuations in their decisions. Basically, to what extent does the actual behaviour (what practitioners do) deviate from optimal values (suggested by theory)? My survey on empirical real options papers shows that the use of a case study is to extract the variables of interest and employ them to solve a valuation problem (I describe some of them in the following discussion). The unanswered question is to what extent people follow real options valuation?

Survey papers confirm that there is such a gap in the literature (see, Cetinkaya and Thiele (2014), Brounen, Jong, and Koedijk (2004), Brunzell, Liljeblom, and Vaihekoski (2013) Savolainen (2016), Kozlova (2017), Lambrecht (2017), Trigeorgis and Tsekrekos (2018)). This study aims to fill this gap and contribute to the literature by setting up a switching options problem for orchards and test if orchardists follow real options strategies in their exercise decisions. These orchardists are active in apple orchards where there is an uncertainty about apple revenue changes in future. ${ }^{2}$ Orchardists allocate their orchards to a specific type of apple or to a combi-

${ }^{1}$ Slade (2001), Purvis, Boggess, Moss, and Holt (1995), Hyde, Stokes, and Engel (2003), Tauer (2006), Tubetov, Musshoff, and Kellner (2012), Shah and Ando (2016), Winter-Nelson and Amegbeto (1998), Cunha-e-sa and Franco (2016), Fackler and Livingston (2002), Kennedy (1986), Williams and Wright (1991), Deaton and Laroque (1992), Anderson and Brorsen (2005), Hagedorn, Irwin, Good, and Colino (2005), H. S. Kim and Brorsen (2012), Song, Zhao, and Swinton (2011), Dixit (1992), Tyner (2008), James, Swinton, and Thelen (2003), Song, Zhao, and Swinton (2011), Livingston, Roberts, and Y. Zhang (2014), Pederson and Zou (2008), Schmit, Luo, and Conrad (2011) and Schmit, Luo, and Tauer (2009), Hauer, Luckert, Yemshanov, and Unterschultz (2016), and Hossiso and Ripplinger (2017).

${ }^{2}$ In this stud, revenue refers to net revenue (profit), otherwise I will clearly state the term gross. 
nation of apple varieties in an analogous fashion to a portfolio. As time goes by, and apple revenues change, they have an incentive to switch their orchards to the more lucrative apple type. I evaluate a situation where the orchardists have the option to change between Braeburn and Queen apple trees by two competing switch strategies: the uproot-plant or the graft strategy. ${ }^{1}$

Grafting is the process of donating a scion, which is a shoot or a bud from another tree, to a rootstock of another tree (the receiver). The uprootplant strategy is relatively more expensive with higher upfront cash cost and a slow process, but results in a rejuvenated orchard, whereas the graft strategy is less expensive and faster but results in carrying on with the existing trees. In this regard, my study not only captures the value of waiting for an investment under uncertainty but also evaluates alternative switch options to optimise the orchardists' land use polices. In other words, the orchardists in each stage of a tree's lifetime have 3 options: to continue with the existing apple trees, to switch to a new type of apple by uprooting the existing apple trees and planting the more lucrative apple type, or to switch by grafting on existing rootstock. In addition, I relax the assumption of irreversibility and allow my orchardists to have the option to reverse their decisions and go back to the old apple type. ${ }^{2}$ The hysteresis effect is revealed through orchardists waiting to exercise the uproot-plant or graft strategies. ${ }^{3}$ For instance, there might be a situation where the orchardists can exercise their option and switch to a more lucrative apple type by uprooting and planting new trees, or by grafting, but they choose to continue with the existing apple trees until the revenues hit the hurdle

\footnotetext{
${ }^{1}$ The choice of two apple types is explained in Chapter 4.

2 I explained in chapter 3 that irreversibility can be discussed in a spectrum from complete reversibility to complete irreversibility depending on the cost of switching. Reversibility in this chapter means to go back to the original apple type but by paying cash cost and time cost.

${ }^{3}$ Hysteresis here means that the switching and implementing decisions will not happen immediately and occurs over time. Therefore, farmers think twice before they switch from one type of apple to another.
} 
rate, which is higher than the Marshallian rule. Hence, I contribute to the literature not only by examining whether practitioners follow real options valuations in practice and how far they are from theoretical values but also by capturing the exact cash cost and time cost of prolonged switching options under uncertainty and reversibility.

In the Section 3.2, I evaluate a situation where representative agents have the option to switch from one product to another, by proposing three models: the cash cost, time cost, and projection models. The assumptions are: (1) products have an infinite lifetime and (2) there is only one switching strategy (removing the existing product and initiating the other product). In contrast, in this chapter, I assume: (1) apple trees have a finite productive lifetime similar to what I see in reality and (2) the model is more complex due to having two competing switch strategies: the uproot-plant and graft strategies. However, I conjecture that the uproot-plant strategy is the optimal policy when orchards are old whereas the graft strategy is optimal when orchards are young. To begin with, I present a short survey of recent papers on empirical real options to clarify how case studies have been utilised in the literature.

Some real options studies are implemented in the mining sector or examine managerial flexibility. For instance, Sina and Guzman (2019) evaluate an open pit mine with two processing method using real options. This model optimises the mine value by assuming commodity price uncertainty and unlimited extraction capacity. They use a numerical example to test their model, and results show that having two processing methods instead of one provide a higher mine value. Rebiasz, Gawel, and Skalna (2017) evaluate the managerial flexibility for steel industry investments. In this study, the case study of a product switch is valued using Monte Carlo simulation. Their results indicate that having the option to diversify products generates a significant value for metallurgical projects . Kim, Ha, and Hyoungkwan (2017) examine managerial flexibility to find the effective 
adaptation strategy in infrastructure using a chooser option. They implement their study on a case study in Seoul and find that having the chooser option increases the project value, which emphasises the strength of real options rather than comparing optimal and actual behaviours. Climate change and renewable energy are other applications of real options. $X$. Wang and Du (2016) study the carbon capture and storage investment decision for a power plant in China. They use their case study to estimate the corresponding model parameters and examine the decision making using both net present value and real options approaches. This study follows a scenario analysis and emphasises on the strength of real options in policy making. M. Zhang, P. Zhou, and D. Zhou (2016) examine investment in renewable energy using a real options approach. They use an application to solar photovoltaic power generation in China to capture their parameters. The authors show that policies such as subsidies, technological progress, and market stability are more important than price volatilities and investment costs.

In the agriculture sector, Ewald, Ouyang, and Siu (2016) employ the real options approach to optimise the harvesting problem (lease vs ownership) for fish farms in Norway. The variables of interest are captured from a newly-established market in salmon futures. The authors show that the existence of salmon futures market and managerial flexibility play key roles for risk averse farmers. In corporate finance, Davis and Cairns (2017) examine a lumpy investment in capital budgeting using irreversible and reversible real options approach. They use the parameters from Brennan and Schwartz (1985) to study completely irreversible investments versus completely reversible investments. A recent paper in investment and disinvestment is Tang, H. Zhou, Chen, K. Wang, and Cao (2017). They study the optimal overseas investment of China using real options under uncertainty and irreversibility. They employ a case study to present the optimal investment through scenario analysis. Results show a higher risk for earlier start-up projects and evaluate the factors that affect on results. My 
study is different from the above mentioned literature by proposing three models based on real option approach and comparing results from theory with my case study in orchards.

The remainder of this chapter proceeds as follows: Section 5.2 describes the horticulture sector in New Zealand and some characteristics of apple orchards. I explain two strategies that are adopted by orchardists to switch from one type of apple to another. Section 5.3 presents the methodology that I follow to analyse the orchardists investment decisions in apple orchards. Section 5.4 proposes empirical results and examines how the optimal land use policy proposed by model is different from what farmers do in practice. Finally 5.5 draws conclusions and discusses the possible solutions and suggestions to the orchardists and authorities to make optimal investments.

\subsection{Apple Orchard Characteristics}

\subsubsection{Horticulture Sector}

According to New Zealand Horticulture (2017), the product of the horticulture sector exceeds $\$ 8.8$ billion in 2017. The dominant products are wine, kiwifruit, and apples. The global demand for New Zealand apples is growing dramatically due to their high quality. As a consequence, apple growers are improving their level of technology and improving practices to keep up with this demand. However, these improvements have made the switching between varieties of apples more frequent than before.

The apple industry in New Zealand developed its commercial role in international markets during the 1910's by establishing the New Zealand Fruitgrowers' Federation (NZFF) and the Fruit Export Control Board (FECB). In recent years apple growers have become concentrated in areas that pro- 
vide higher returns due to comparative advantage compared with other regions. In this regard, the Hawke's Bay and Nelson regions account for almost 5115 ha (60\%) and 2496 ha (30\%) of total land (8845 ha) that is allocated for growing apples (Horticulture, 2017). The Hawke's Bay is a region with fertile soils, high sunshine, and great climate for agriculture (ANZ Agri Focus, 2015). The current situation makes this region an ideal case study to examine the orchardists' behaviour in terms of exercising switching options.

There are a wide range of factors that drive apple growers to switch to new apple types. For example, the demand or market for specific types of apple, available government financing and etc. However, it is not always possible to invest and redevelop any further in large scale orchards due to financial and time constraints as well as missing the cash flow stream. Therefore, orchardists look for an adjustment such as grafting or modifying training and pruning techniques for existing trees (MPI, 2017). Originally, orchardists used to plant trees that grew slowly but eventually became big and tall due to wide spacing. As time changed, new standards have come through and competition pushes orchardists to choose a type of rootstock that allows them to grow trees much more closely and naturally have them not so tall with no shade, higher density of trees, and higher productivity. This also saves on labour costs due to the trees being much easier to spray, trim, and pick without need for ladders. The strategy of having high density orchards is one of the common management systems that help growers to profit from high early production. Orchardists can speed up the time to become productive by using a dwarf or semi-dwarf rootstock. 


\subsubsection{The Uproot-Plant Versus The Graft}

Orchardists have the option to uproot old apple trees quickly and cheaply, then plant new trees very slowly and expensively due to the high upfront cash cost of planting and the time required for the trees to reach maturity. The alternative way is to graft branches onto an existing tree. This is less expensive and faster but continues with the existing trees. In the grafting process, the new scions grow up on old trees and save time as they use the original root systems. The yield during the early year of a tree's lifetime depends on a wide range of factors. However, the relationship between tree density and cumulative yield follows the law of diminishing returns. In the first stage of planting new trees, there is an upward sloping relationship then it follows a constant relationship. In other words, adding more density will not increase the yield to compensate the extra unit cost. Moreover, some other variables such as water availability, soil type, climate, weather, elevation, direction of slope, irrigation system, and diseases will affect on productivity. Assuming all the conditions to be constant, the cash flows throughout the trees' lifetime depend on the age and how far they are on to the grafting process (if there is any graft). Table 5.1 demonstrates the cash flows of the two competing strategies throughout a tree's lifetime.

Table 5.1 shows that the productivity of an apple tree grows gradually until it reaches full productivity. While the apple trees approach to the end of their lifetime, they lose their productivity gradually. However, in the interim, an orchardist might use the uproot-plant or graft strategies. The uproot-plant strategy is slower in reaching full productivity than the graft strategy. The uproot-plant strategy begins with no productivity in the first year and is expected to grow at $25 \%$ in the following years, with the productivity increasing by $25 \%$ per year until the productivity reaches to $100 \%$. Similarly, the graft strategy follows the same process but the productivity begins with $25 \%$ in the first year and reaches full productivity 
TABle 5.1: Apple Trees Cash Flows Throughout Their Lifetime Using the Uproot-Plant and the Graft Strategy.

This table shows the productivity of an apple tree throughout its lifetime according to 3 options. The productivity grows gradually according to the age of the apple tree until it reaches full productivity, when orchardist keep the existing trees. Note that the productivity decreases gradually as apple trees approach to the end of their productive lifetime. If orchardists uproot the existing trees, they lose the productivity for the first year and then according to the tree's age it increases gradually again. In the grafting option production is a fraction of tree's productivity at that age and depends how far the process is on to the grafting process.

\begin{tabular}{|c|c|c|c|c|c|}
\hline Option & & & Productivity in Lifetime & & \\
\hline Keep $(g(t))$ & $0 \% \quad 25 \%$ & $50 \%$ & $75 \% 100 \% 100 \% 100 \% 100 \% 100 \% 100 \%$ & . & $0 \%$ \\
\hline Uproot & $\ldots$ & $\cdots$ & $0 \% \quad 25 \% \quad 50 \% \quad 75 \% \quad 100 \%$ & . & $0 \%$ \\
\hline Graft $(h(t))$ & $\ldots$ & $\cdots$ & $\begin{array}{llllll}\ldots & 25 \% & 50 \% & 75 \% & 100 \% & 100 \%\end{array}$ & $\ldots$ & $0 \%$ \\
\hline
\end{tabular}

one year earlier. The orchardists can apply graft at any age of productive apple trees. Hence, the trees' production depends on the trees' age and how far they are on to graft process. Apple trees in both strategies begin to lose productivity as they approach to the end of their lifetime until they die. One would expect the orchardists prefer to employ the graft strategy when trees are young and uproot them when they are old. Table 5.2 shows a summary of initial costs to establish an orchard. The cost varies based on the root stock, soil quality, water availability and etc.

\subsection{Methodology}

In this chapter, I look at a real options switching problem where the orchardists have blocks of land and have the option to grow different types of apples. I examine the orchardists' behaviour in the Hawke's Bay region, where they switch from one type of apple to another according to apple revenue changes. I focus on Braeburn and Queen types due to their higher switch frequency and more volatile revenues in the dataset. The 
TABLE 5.2: Initial Cost to Establish an Orchard.

This table presents a summary of initial costs to establish an orchard. These costs can change depending on the root stock category, soil quality, water availability and etc.

\begin{tabular}{lll}
\hline Item & $\$ /$ Tree & $\$ /$ ha \\
\hline Trees & $\$ 40-\$ 50$ & $\$ 16000-\$ 20000$ \\
Planting Costs (Labour \& equipment) & $\$ 15-\$ 18$ & $\$ 6000-\$ 7000$ \\
Fertiliser & $\$ 2.50-\$ 5.00$ & $\$ 1000-\$ 2000$ \\
Mulch & $\$ 7.30$ & $\$ 2900$ \\
Tree shelter & $\$ 15$ & $\$ 6000$ \\
Orchard shelter & $\$ 3.10$ & $\$ 1250$ \\
Total & $\$ 83-\$ 98$ & $\$ 33150-\$ 39150$ \\
\hline Site Specific Costs & & \\
Irrigation & $\$ 13-\$ 20$ & $\$ 5000-\$ 8000$ \\
Drainage & $\$ 5-\$ 10$ & $\$ 2000-\& 4000$ \\
Contouring & $\$ 13-\$ 25$ & $\$ 5000-\$ 10000$ \\
Total & $\$ 113-\$ 153$ & $\$ 45150-\$ 61150$ \\
\hline
\end{tabular}


orchardists plant Braeburn apple trees, which generates revenue $S_{B}$, or Queens, with revenue $S_{Q}$. However, with the passage of time and changes in competing apple revenues, they find themselves in a situation where they have the option to switch to a more lucrative apple type.

I assume a complete market with two agents. The role of risk aversion depends on whether the market is complete. In a complete market, all products are tradable and agents maximise the value of their assets and work with risk neutral probabilities. In contrast, in an incomplete market, all products are not tradable and one should work with utility function, marginal utility, consumption, endowments and etc. Also, I consider orchardists who have access to a parcel of land. At any point in time, this parcel of land can be allocated in a variety of states, which will be discussed subsequently below. The land value in all cases depends on the current value of two apple types. The underlying assumption here is that apple revenues follow geometric Brownian Motions (GBMs) as follows:

$$
\begin{aligned}
& d S_{B}=\mu_{B} S_{B} d t+\sigma_{B} S_{B} d z_{B} \\
& d S_{Q}=\mu_{Q} S_{Q} d t+\sigma_{Q} S_{Q} d z_{Q},
\end{aligned}
$$

where $d S_{B}$ and $d S_{Q}$ represent apple revenue changes, $\mu_{B}$ and $\mu_{Q}$ are apple drifts, $S_{B}$ and $S_{Q}$ are apple revenues, $\sigma_{B}$ and $\sigma_{Q}$ are volatilities of apple prices, $d t$ is the small interval of time, and finally $d z_{B}$ and $d z_{Q}$ are increments of Brownian motions (that have correlation $\rho_{B Q}$ ). The land value in my problem is derived based on the apple revenue processes (5.1) over time. In this chapter, I embellish my model from the third chapter, where 
land value is governed by the operator $\mathcal{L}$.

$$
\begin{aligned}
0 & =\frac{\partial V_{i}}{\partial t}+\mu_{B} S_{B} \frac{\partial V_{i}}{\partial S_{B}}+\mu_{Q} S_{Q} \frac{\partial V_{i}}{\partial S_{Q}}+\frac{1}{2} \sigma_{B}^{2} S_{B}^{2} \frac{\partial^{2} V_{i}}{\partial S_{B}^{2}}+\frac{1}{2} \sigma_{Q}^{2} S_{Q}^{2} \frac{\partial^{2} V_{i}}{\partial S_{Q}^{2}} \\
& +\rho \sigma_{B} S_{B} \sigma_{Q} S_{Q} \frac{\partial^{2} V_{i}}{\partial S_{B} \partial S_{Q}}-r V_{i}+C F_{i} \\
& \equiv \frac{\partial V_{i}}{\partial t}+\mathcal{L} V_{i}+C F_{i},
\end{aligned}
$$

where $V_{i}$ is the land value in state $i, \mathcal{L}$ is the operator:

$$
\begin{aligned}
\mathcal{L V} & =\mu_{B} S_{B} \frac{\partial V}{\partial S_{B}}+\mu_{Q} S_{Q} \frac{\partial V}{\partial S_{Q}}+\frac{1}{2} \sigma_{B}^{2} S_{B}^{2} \frac{\partial^{2} V}{\partial S_{B}^{2}}+\frac{1}{2} \sigma_{Q}^{2} S_{Q}^{2} \frac{\partial^{2} V}{\partial S_{Q}^{2}} \\
& +\rho \sigma_{B} S_{B} \sigma_{Q} S_{Q} \frac{\partial^{2} V}{\partial S_{B} \partial S_{Q}}-r V,
\end{aligned}
$$

$\rho$ is the correlation between apple types, $r$ is the discount rate, and the $C F_{i}$ is an extra term compared with a conventional option pricing formula, that captures the revenues generated in state $i$. I employ the Peaceman and Rachford (1955) alternating direction implicit (ADI) method to solve the PDE (5.2) and find the land value in different states of activity (see 3.24 and 3.25). In the third chapter (3.5), the cash flows depend only on the states. In contrast, in this chapter, the cash flows are more complex and not only depend on the states but also on the age of apple trees and the switching strategy. I define the cash flows as follows:

$$
\begin{aligned}
& C F_{i}\left(t, y_{B}, y_{Q}\right)=g(t) e^{\left(e_{i B} y_{B}+e_{i Q} y_{Q}\right)} \\
& C F_{i}^{g r a f t}\left(t, b, y_{B}, y_{Q}\right)=g(b+t) h(b) e^{\left(e_{i B} y_{B}+e_{i Q} y_{Q}\right)},
\end{aligned}
$$

where $g(t)$ is the fraction of a fully productive cash flow that a $t$ years old tree generates, $C F_{i}^{\text {graft }}\left(t, b, y_{B}, y_{Q}\right)$ is the cash flow of a tree that is $b+t$ years old and $b$ years into a graft, $h(t)$ is the fraction due to grafting, and $e_{i B} y_{B}+$ 
$e_{i Q} y_{Q}$ is a term derived from transformed apple prices times eigenvalues.

In this chapter, I set up a dynamic optimisation model to find the optimal land value. The model examines the orchardists' behaviour in an uncertain environment where they allocate land for growing one of two competing apple types, Braeburn or Queen. However, there are situations where the orchardists realise having apple trees of the other type is more lucrative than their current type. In the model, there are 6 states for the land: two productive states, two unproductive states, and two grafting states. Orchardists in productive states B and Q, grow apple types Braeburn or Queen. The unproductive states consist of the Transition state where the land has no trees on it and the Deceased state where the land has unproductive (dying) trees. Finally, the grafting states entail grafting Braeburn or Queen branches on to existing trees. In the following, I describe each state and the corresponding strategy for the orchardists. I begin with unproductive states (Transition and Deceased) as they are the beginning and the end of lifetime of a tree and account for terminal values later in my discussion.

Transition State is an unproductive state where the orchardists have land with no apple trees but are ready to plant either Braeburn or Queen apple trees. The transition land value solves:

$$
\begin{aligned}
& \mathcal{L} V_{T r} \leq 0 \\
& V_{T r} \geq V_{B, p}(0)-K_{B, p} \\
& V_{T r} \geq V_{Q, p}(0)-K_{Q, p},
\end{aligned}
$$

where $V_{T r}$ is the value of the land in the transition state, $V_{B, p}(0)$ and $V_{Q, p}(0)$ are the values of the land where Braeburn and Queen apple types have just been planted respectively (but have not yet reached productivity), $K_{B, p}$ and $K_{Q, p}$ are the upfront cash costs of planting apple types Braeburn and Queen respectively. In the transition state, the orchardists' options are to plant Braeburn apple trees or to plant Queen apple trees. The planting is 
relatively expensive but is instantaneous, and results in young (unproductive) apple trees with a long life ahead of them.

Deceased State is the other unproductive state where orchardists have land with unproductive old apple trees (age above $T^{D i e}$ ) that generate no cash flows. In this state, the only option available is to uproot the trees and convert the land into the transition state. The land value in this state obeys:

$$
\begin{aligned}
& \mathcal{L} V_{D} \leq 0 \\
& V_{D} \geq V_{T r}-K_{u},
\end{aligned}
$$

where $V_{D}$ is the deceased land value, and $K_{u}$ is the upfront cash cost of uprooting that is the same for both tree types.

Productive States are situations where orchardists have productive apple trees growing Braeburn or Queen apples. Orchardists receive cash flows from Braeburn in the first productive state and Queen in the second productive state. Productive states are represented as a continuum of states accounting for the tree's age. Therefore, the land values are conditional on tree's type and age. I compute the land use value at each age of apple trees with the deceased land value $\left(V_{D}\right)$ accounting for the terminal value (I solve my dynamic model by backward induction). Orchards in this state have two options, one to uproot the trees, and the second to begin grafting. The land value solves:

$$
\begin{aligned}
& V_{i, p}\left(T^{D i e}\right)=V_{D} \\
& \mathcal{L} V_{i, p}(t)+C F_{i}\left(t, y_{B}, y_{Q}\right)+\frac{\partial V_{i, p}}{\partial t} \leq 0 \\
& V_{i, p}(t) \geq V_{T r}-K_{i, u} \\
& V_{i, p}(t) \geq V_{j, g}(0, t)-K_{j, g},
\end{aligned}
$$

where $V_{i}(t)$ is the planted land value at age $t, K_{i, u}$ is the upfront cash cost 
of uprooting in state $i, V_{j, g}(0, t)$ is the value of the land with a fresh graft on a $t$ year old tree, and $K_{j, g}$ is the upfront cash cost of grafting with type $j$ apples.

The orchardists have two alternative strategies to switch from one type of apple to another: uprooting and planting new trees, or by grafting. They uproot their trees by paying the upfront cash cost of uprooting and moving to the transition state, where land is ready for planting either apple trees (see equation (5.4)). Alternatively, an orchardist can graft new branches on to existing rootstock. Technically, this process is relatively less expensive and faster than the uproot-plant strategy but continues with the existing trees. If the orchardists have lands with Braeburn apple trees, and realise Queens are more lucrative, they can graft Queen branches on to the rootstock of their existing apple trees. The orchardists pay the upfront cash cost of grafting and receive reduced cash flows for $T^{\text {graft }}$ years until the apple trees reach full productivity. By the time the grafting process is completed, the orchardists have apple trees of the alternative type whose age is $T^{\text {graft }}$ years older. The value of the land in the beginning of the graft on to a tree with $b$ years age is $\left(V_{i, g}(0, b)\right)$, where land with grafted trees obeys:

$$
\begin{aligned}
& V_{i, g}\left(T^{\text {graft }}, b\right)=V_{i, p}\left(b+T^{\text {graft }}\right) \\
& \mathcal{L} V_{i, g}(t, b)+C F_{i}^{\text {graft }}\left(t, b, y_{B}, y_{Q}\right)+\frac{\partial V_{i, g}(t, b)}{\partial t} \leq 0 \\
& V_{i, g}(t, b) \geq V_{T r}-K_{u} \\
& V_{i, g}(t, b) \geq V_{j, g}(0, b+t),
\end{aligned}
$$

where $b$ is the tree's age at the start of grafting, $t$ is the time spent grafting, $V_{i, g}(t, b)$ is the value of the land with grafted trees, $V_{i, p}\left(b+T^{g r a f t}\right)$ is the value of the land with $b+T^{\text {graft }}$ year old trees that accounts for the terminal value of grafting, and $V_{j, g}\left(0, b_{t}\right)$ is the value of the land if the orchardist started grafting the other apple type when the tree was age $b+t$. 
Note that I use the ADI method to solve the system of equations in (5.4) and (5.5) (transition and deceased states), which are elliptic problems whereas, (5.6) and (5.7) (productive states), are parabolic problems. I solve (5.4) and (5.5) by including a $\frac{\partial V}{\partial t}$ term but iterating until this becomes 0 .

Intuitively, the orchardists switch from one type of apple to another only if the competing apple type is sufficiently lucrative to cover the cash cost and the time cost of switching. Both the uproot-plant and graft strategies entail a long period of time before reaching fully productive apple trees. The uproot-plant strategy is more expensive and slower to reach full productive apple trees, but has the advantage of rejuvenating the orchard. In contrast, the graft strategy is relatively less expensive and faster to reach full production due to keeping the rootstock of existing apple trees but continues with the existing trees. However, neither the uprootplant nor the graft strategy is able to suppress the level of uncertainty and the time cost of switching. The level of uncertainty for options to switch is higher in horticulture compared with other sectors due to a prolonged switching process. When orchardists switch from one type of apple to another, they lose the existing cash flows and by the time they complete the process, apple revenues might no longer be in their favour. This model is able to capture the exact time cost of this process. Orchardists in the model have the option to reverse the switching process at any stage of the process. However, reversing the switching process and going back to the existing apple type (either by grafting or uprooting) is again time consuming.

\subsection{Empirical Results}

In this section, I report the results of my switching real options model. The model outputs entail the land value for each apple type (Braeburn and Queen) at each age according to keeping the existing apple trees (Tval), 
switching to the competing apple type by the uproot-plant strategy (Uval), switching by the graft strategy (Gval), and the optimal land value (highest value across available options). My results demonstrate the optimal exercise frontiers and what policy (Tval, Uval, and Gval) is ideal when apple revenues change. I compare optimal land value from my switching real options problem with what value orchardists achieve in the orchards and examine how their choice is different from the model. Employing the conditional logit regression model helps us to test the behaviour of orchardists, conditional on the money at stake along with their size.

Table 5.3 presents the set up parameters for apple types Braeburn and Queen. It costs $\$ 10000$ per hectare and 3 months to uproot and costs $\$ 100000$ per hectare to plant each apple type. ${ }^{1}$ In contrast, grafting costs $\$ 35000$ in 4 years, which is faster and less costly. Braeburn and Queen have drifts of $4 \%$ and $6 \%$ with volatilities of $8 \%$ and $13 \%$ respectively. The correlation between two apple types is 0.26 in an economy with a $7 \%$ discount rate.

Each apple tree in my model generates three output results: Tval, Uval, and Gval. All three outputs are generated over the age of apple trees from 1 year to 34 years. Hence, there are 204 different output for each orchard-time observations with two apple types each with 34 years and 3 different situations (Tval, Uval, Gval). Also, the results include transition and deceased states. Table 5.4 demonstrates the summary of outputs model. On average, apple trees in the sample are in their middle age and full productivity. Comparing the available land use values (Tval, Uval, Gval) shows that on average, keeping the existing trees has the highest land value with less deviation from its mean compared with switching to competing apple type (either the uproot-plant or the graft). Results show that the strategy chosen by orchardists is different from the optimal policy of the model. However, it is not possible to reject the choice of orchardists

\footnotetext{
${ }^{1}$ In this study, I assume that all apple varieties require the same cost and time to grow and have the same productivity.
} 
TABLE 5.3: Queen and Braeburn Apple Types Parameters.

Note: This table shows the parameters for apple types Queen and Braeburn in my analyses. The variables of interest are: cost, time, volatility, drift, discount rate, and correlation. All values are scaled based on thousands dollar per hectare in years.

\begin{tabular}{lccc}
\hline Parameters & Symbol & Braeburn & Queen \\
\hline Cost (uproot, plant) & $K_{u, p}$ & $(10,100)$ & $(10,100)$ \\
Time (uproot, plant) & $T_{u, p}$ & $(0.25,5)$ & $(0.25,5)$ \\
Cost (Graft) & $K_{g}$ & 35 & 35 \\
Time (Graft) & $T_{g}$ & 4 & 4 \\
Volatility & $\sigma$ & $8 \%$ & $13 \%$ \\
Drift & $\mu$ & $4 \%$ & $6 \%$ \\
Discount Rate & $r$ & $7 \%$ & \\
Correlation & $\rho$ & 0.26 & \\
\hline
\end{tabular}

due to having assumptions in the model set up, and the psychological environment. Also, orchardists may diversify their orchards across different apple varieties that affects on their decisions. The model implies that minimum and maximum percentage difference dollar value is $0 \%$ and $8.4 \%$ per hectare respectively and on average, $0.5 \%$ per hectare. The amount of percentage difference dollar value for orchardists varies based on the land area and the price of apple type in that year. Orchardists pick 3104 tray carton apples in each hectare and Braeburn apple gross revenues vary from $\$ 54186.28$ to $\$ 86485.62$ whereas Queen apple gross revenues vary from $\$ 64596.38$ to $\$ 148754.60$ per hectare over years $2006-16$ respectively (see Chapter 4). Hence, according to the current model set up, on average an orchardist with a hectare land area loses $0.5 \%$ of the corresponding apple net revenue. Assuming the same cost of $\$ 50$ for both apple types, on average the loss varies varies from $\$ 220.93$ to $\$ 382.43$ for Braeburn and from $\$ 272.98$ to $\$ 693.77$ for Queen.

Figure 5.1, shows the optimal land use policy for Braeburn and Queen 
TABLE 5.4: Sample Results of Dynamic Optimisation.

This tables presents the results of land use optimisation. I report key variables: age of apple trees (Age), land use for keeping the existing apple trees type 1 or 2 (Tval), land use for the competing apple type by using the uproot-plant strategy (Uval), land use for the competing apple type by using the graft strategy (Gval), optimal value amongst all 3 options (optimal), the percentage difference dollar value between the optimal land use and what orchardists choose (Diff), and finally the land area (Area). All values are scaled based on thousands dollar and hectares.

\begin{tabular}{lcccrr}
\hline \multicolumn{1}{c}{ Variable } & N Obs & Mean & Std Deviation & Min & Max \\
\hline Age & 5188 & 17.24 & 7.95 & 1.00 & 33.00 \\
Keep the Existing Trees Value (Tval) & 5188 & 2283.50 & 347.47 & 1555.62 & 2634.67 \\
Uproot-Plant Value (Uval) & 5188 & 2109.36 & 318.57 & 1771.15 & 2505.03 \\
Grvaft Value (Gval) & 5188 & 2187.60 & 243.85 & 987.95 & 2653.04 \\
Optimal & 5188 & 2301.80 & 333.83 & 1271.15 & 2634.67 \\
(Optimal-Actual)/Actual & 5188 & 0.005 & 0.015 & 0.000 & 0.084 \\
Area & 5188 & 1.02 & 1.25 & 0.02 & 22.58 \\
\hline
\end{tabular}

at age 5, 15, 25, and 33 years old. In this figure, orchardists grow Braeburn apple type ( $x$ axis) and Queen is the competing type ( $y$ axis), which is more lucrative. The axes are dollar value prices of apple types, scaled on 0 to 30 dollars. Orchardists receive the Braeburn revenues over time by keeping the existing trees. However, when the competing apple type (Queen) is more lucrative, they have the incentive to switch either by the uproot-plant or the graft strategy ( $y$ axis). The former is an expensive and slow process but results in a rejuvenated orchard while the latter is less expensive and faster but continues with the existing trees. Having said that, orchardists exhibit inertia, keeping their existing apple type and not exercising their options except when the competing apple type is really in the money. Orchardists have a hurdle rate in their mind and they exercise their option when revenues hit the exercise frontier. Figure 5.1 shows that when orchardists have orchards with 5-year old apple trees they are more likely to keep the existing apple tree (Braeburn) but when the Queen type is more lucrative, they exercise their option and switch by grafting. As apple trees become older and they are 15-years old, the grafting becomes 

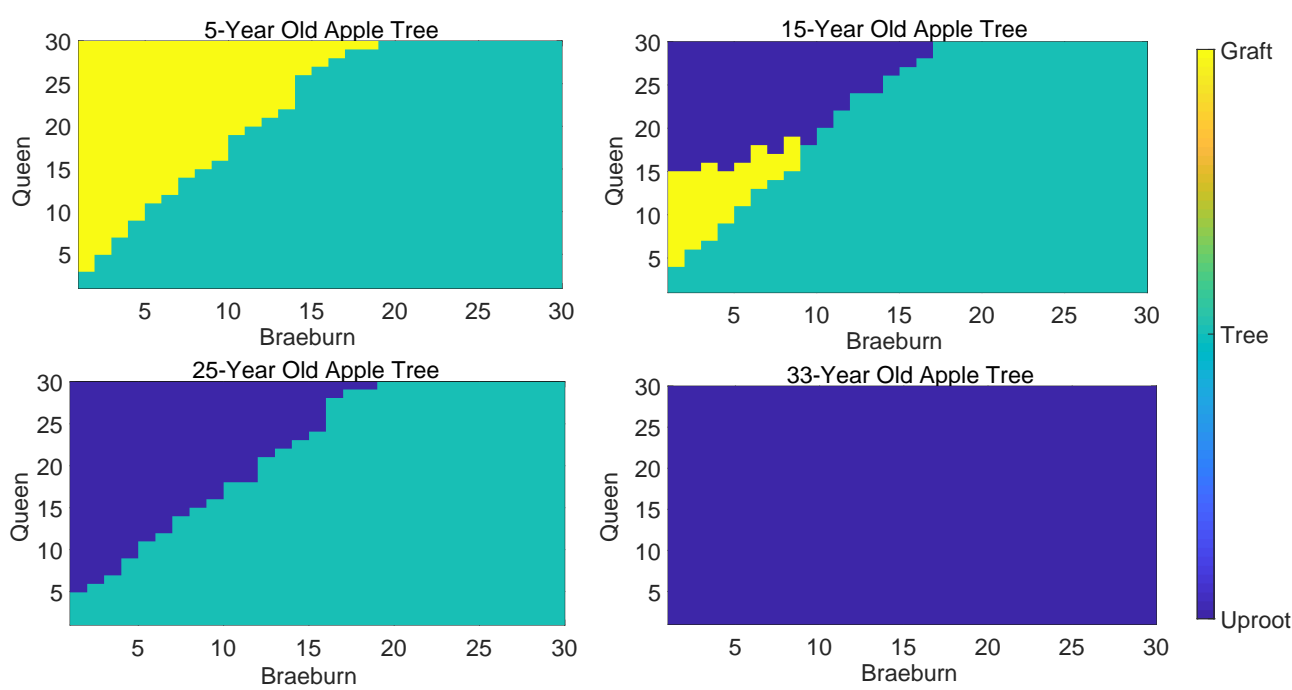

FIGURE 5.1: Optimal Land Use Policy.

This figure shows orchardists who grow Braeburn apples and optimise land use policy as the apple net revenues change over time. Orchardists look at the net revenues of both apple types and decide to keep the existing apple trees or switch to the competing apple type. In this figure, as the Braeburn net revenues grow (corresponding land value on $x$ axis) orchardists prefer to keep the existing apple trees. However, looking at Queens net revenues (corresponding land value on $y$ axis) shows that when net revenues hit the hurdle they are willing to switch to the competing apple type. For a 5-year old apple tree the switch is through the graft strategy, but as the trees become older (25-year) and Queen is more lucrative, the uproot-plant becomes a better alternative way to switch having the advantage of rejuvenating the orchard. As apple trees near their unproductive age, grafting is not an ideal switching strategy any more and uprooting is the optimal policy. By the time trees die (33-years), the optimal policy is to uproot them and plant new trees. Note that the land value of apple types on each axis are scaled on 30 dollars value.

the ideal strategy for switching. Note that both switch strategies are available throughout the life of apple trees and the choice of switch strategy is driven by two key factors: the profitability of alternative apple type, and the age of apple trees. Keeping the existing apple type (Braeburn) is more likely throughout the productive life of apple trees. Finally, apple trees at age 33-years old become unproductive and die. Therefore, the optimal policy is to uproot unproductive apple trees and have a rejuvenated orchard. Overall, results indicate that orchardists rarely switch.

I use a conditional logit model to examine orchardists' choices while the choice properties vary across orchardists in my sample data. The conditional logit model is similar to logistic regression but individuals are subject to different situation before they select their choices. In this regard, 
I offer them the choice of keeping the existing apple trees, uprooting and planting young apple trees, and grafting on the existing apple trees, while using the size of land as a condition. Equation (5.8) presents the conditional logit model set up.

$$
U_{\text {choice }}=\beta_{1} \text { Val }_{\text {choice }}+\beta_{2} D_{\text {Tree }}+\beta_{3} D_{\text {Tree }} * D_{\text {size }}
$$

where $U_{\text {choice }}$ is the orchardists choice value, $V a l_{\text {choice }}$ is what policy they adopt whether to keep the existing trees or employ a switching strategy by uproot-plant or graft, $D_{\text {Tree }}$ is a dummy for keeping the exiting apple trees that is 1 if it is tree and 0 otherwise, and finally $D_{\text {size }}$ is the dummy variable for the land size that is one if it is above 2.02 hectare and 0 otherwise.

Table 5.5 shows the results of logit model. I examine the attributes of orchardists' choice by having orchardists' choice as the response variable and land values, dummy variable for keeping existing trees, and the interaction of keeping existing trees with the large orchardist dummy variable as explanatory variables. The orchardists' choices as the response variable are 1,2 , or 3 , which are keeping the existing trees, switching to the competing apple type using the uproot-plant strategy, and switching by employing the graft strategy respectively. The dummy variable for the situations where orchardists keep the existing trees is 1 and 0 otherwise. To test how this choice might be different between small orchardists versus large orchardists, I define a dummy variable based on the land size. If orchardists' land area falls in the top $10 \%$ of sizes (above 2.02 ha) dummy is 1 and 0 otherwise. I also include the interaction of dummy for keeping the existing apple trees and large orchardists in my regression to test if they are biased in favour of any choice. My results show that orchardists are in favour of keeping the existing apple trees rather than switching to the competing apple type, using the uproot-plant or graft strategies. Results are significant at the $1 \%$ level. However, comparing my results for small orchardists versus large orchardists do not reveal any significant re- 
TABLE 5.5: Conditional Logistic Regression Results.

This table presents the results of orchardists' choice of land use when the attributes of choice vary across orchardists. I run a conditional logit regression model and report the estimate values, standard errors and corresponding P-values. The first variable entails the corresponding values of keeping the existing apple trees, switching by the uproot-plant strategy, and switching by the graft strategy. The second variable is the dummy variable that is 1 if orchardists keep the existing trees and 0 otherwise. Finally, the third variable is the interaction of the dummy variable for keeping the existing apple trees and the large size land dummy. The land area above $2.02 \mathrm{ha}$ (top $10 \%$ area) accounts for large orchardists. Hence, the dummy is 1 if the land area is above 2.02 ha and 0 otherwise

\begin{tabular}{lcc}
\hline \multicolumn{1}{c}{ Parameters } & Estimate & P-Value \\
\hline Land Values & 0.0037 & 0.2303 \\
Tval Dummy & $5.2578^{* * *}$ & $<.0001$ \\
Tval Dummy*Large Orchardist Dummy & 17.5087 & 0.9960 \\
\hline
\end{tabular}

sults. Therefore, the bias towards inertia is not more significant for small orchardists compared with large orchardists.

\subsubsection{Sensitivity Analysis}

An Increase in uproot-plant Cash Cost decreases the probability of switches by this strategy due to higher switching costs. I increase the cash cost of uprooting from $\$ 10000$ to $\$ 15000$ and planting from $\$ 100000$ to $\$ 150000$ respectively. Table 5.6 presents the results after increasing the uproot-plant cash cost. Results show that on average keeping the existing trees has the highest value compared with the uproot-plant and graft strategies. The reason is the higher cash cost to pay and the time cost to wait. On average the value of graft strategy is more than the uproot-plant strategy due to increasing the cash cost of switching. After an increase in cash cost, on average, the percentage difference dollar value between the optimal and actual values decreases from $0.5 \%$ to $0.2 \%$ that might be due to having less switches after increasing the cash cost. In this case, by assuming the same cost of $\$ 50$ for both apple types, orchardists lose between $\$ 58.37$ 
TABLE 5.6: Uproot-Plant Cash Cost Change Sensitivity.

This tables presents the results of land use optimisation when the uproot cash cost increases from $\$ 10000$ to $\$ 15000$ and the plant cash cost from $\$ 100000$ to $\$ 150000$ respectively. I report key variables: land use for keeping the existing Braeburn or Queen apple trees (Tval), land use for the competing apple type by using the uproot-plant strategy (Uval), land use for the competing apple type by using the graft strategy (Gval), optimal value amongst all 3 options (optimal), and finally the percentage difference dollar value between the optimal land use and what orchardists choose (Diff). All values are scaled based on thousands dollar and hectares.

\begin{tabular}{lccccr}
\hline \multicolumn{1}{c}{ Variable } & N Obs & Mean & Std Deviation & Min & Max \\
\hline Keep the Existing Trees Value (Tval) & 5188 & 911.54 & 158.87 & 461.68 & 1268.94 \\
Uproot-Plant Value (Uval) & 5188 & 713.62 & 162.38 & 567.13 & 1013.19 \\
Grvaft Value (Gval) & 5188 & 822.62 & 206.33 & 167.93 & 1229.95 \\
Optimal & 5188 & 932.62 & 158.73 & 567.13 & 1268.94 \\
(Optimal-Actual)/Actual & 5188 & 0.002 & 0.07 & 0.00 & 0.50 \\
\hline
\end{tabular}

and $\$ 122.92$ per hectare for Braeburn and between $\$ 179.19$ and $\$ 247.51$ per hectare for Queen over years 2006-16.

Increasing time cost is another key variable that affects on orchardists switches. I increase the time cost of uprooting from 3 months to 6 months and planting time from 5 years to 7 years. Table 5.7 presents the results after increasing the uproot-plant time cost. On average, keeping the existing trees has the highest value compared with the switching strategies (Uval and Gval). On average the value of graft strategy is more than the uproot-plant strategy due to increasing the time cost of switching. On average the percentage difference dollar value between the optimal and actual values decreases from $0.5 \%$ to $0.15 \%$ (compared with the baseline scenario) which could be due to predicting less switches and keeping the existing apple trees after increasing the time cost. In this case, by assuming the same cost of $\$ 50$ for both apple types, orchardists lose between $\$ 31.28$ and $\$ 79.69$ per hectare for Braeburn and between $\$ 46.89$ and $\$ 173.13$ per hectare for Queen over years 2006-16.

A decrease in drift is the next sensitivity scenario. Table 5.8 presents 
TABLE 5.7: Uproot-Plant Time Cost Change Sensitivity.

This tables presents the results of land use optimisation when the uprooting time cost increases from 3 months to 6 months and planting time cost form 5 years to 7 years respectively. I report key variables: land use for keeping the existing Braeburn or Queen apple trees (Tval), land use for the competing apple type by using the uproot-plant strategy (Uval), land use for the competing apple type by using the graft strategy (Gval), optimal value amongst all 3 options (optimal), and finally the percentage difference dollar value between the optimal land use and what orchardists choose (Diff). All values are scaled based on thousands dollar and hectares.

\begin{tabular}{lcccrr}
\hline \multicolumn{1}{c}{ Variable } & N Obs & Mean & Std Deviation & Min & Max \\
\hline Keep the Existing Trees Value (Tval) & 5188 & 842.44 & 156.38 & 370.53 & 1144.25 \\
Uproot-Plant Value (Uval) & 5188 & 610.73 & 134.28 & 489.70 & 939.27 \\
Grvaft Value (Gval) & 5188 & 695.45 & 171.47 & 103.52 & 1151.02 \\
Optimal & 5188 & 860.62 & 142.72 & 489.70 & 1151.02 \\
(Optimal-Actual)/Actual & 5188 & 0.0015 & 0.088 & 0.00 & 0.63 \\
\hline
\end{tabular}

the results when Braeburn drift decreases from $4 \%$ to $2 \%$ and Queen drift decreases from $6 \%$ to $4 \%$. In this scenario, on average, the value of keeping the existing apple trees is more than employing the uproot-plant or graft strategies. On average the graft value is more than the uproot-plant value. A decrease in drift (growth rate) decreases the probability of switches between apple types due to a negative impact on return of apple types. Cash cost and time cost of switching along with less return (growth rate) encourage orchardists to keep the existing apple trees. On average, the percentage difference dollar value between the optimal and actual values decreases from $0.5 \%$ to $0.3 \%$ (compared with the baseline scenario). In this scenario, by assuming the same cost of $\$ 50$ for both apple types, on average, orchardists lose between $\$ 112.56$ and $\$ 209.38$ per hectare for Braeburn and between $\$ 143.79$ and $\$ 396.26$ per hectare for Queen over years 2006-16.

Volatility sensitivity evaluates a situation where the baseline volatility parameters increase. Table 5.9 presents the results when Braeburn volatility increases from $8 \%$ to $12 \%$ and Queen volatility increases from 
TABle 5.8: Drift Change Sensitivity.

This tables presents the results of land use optimisation when drift decreases from $4 \%$ and $6 \%$ in Table 5.4 to $2 \%$ and $4 \%$ for Braeburn and Queen apple types respectively. I report key variables: land use for keeping the existing Braeburn and Queen apple types (Tval), land use for the competing apple type by using the uproot-plant strategy (Uval), land use for the competing apple type by using the graft strategy (Gval), optimal value amongst all 3 options (optimal), and finally the percentage difference dollar value between the optimal land use and what orchardists choose (Diff). All values are scaled based on thousands dollar and hectares.

\begin{tabular}{lcrrrr}
\hline \multicolumn{1}{c}{ Variable } & N Obs & Mean & Std Deviation & Min & Max \\
\hline Keep the Existing Trees Value (Tval) & 5188 & 1007.24 & 171.61 & 611.90 & 1405.56 \\
Uproot-Plant Value (Uval) & 5188 & 918.45 & 187.74 & 750.25 & 1264.99 \\
Grvaft Value (Gval) & 5188 & 962.37 & 232.63 & 247.84 & 1434.16 \\
Optimal & 5188 & 1036.75 & 184.81 & 750.25 & 1434.16 \\
(Optimal-Actual)/Actual & 5188 & 0.003 & 0.066 & 0.00 & 0.27 \\
\hline
\end{tabular}

$13 \%$ to $17 \%$. Results show that on average keeping the existing apple trees has a higher value than the uproot-plant and graft strategies. However, the graft strategy has a higher value than the uproot-plant strategy. An increases in volatility provides more time value to the options and results in orchardists being more reluctant to exercise their switching option. In this scenario, by assuming the same cost of $\$ 50$ for both apple types, on average, orchardists lose between $\$ 96.30$ and $\$ 183.44$ per hectare for Braeburn and between $\$ 124.41$ and $\$ 351.64$ per hectare for Queen over years 2006-16.

An Increase in the discount rate will increase the orchardists' expectation of future returns over the course of time. Hence, when they find a switching option, they are willing to wait and see if it gets even better. Table 5.10 presents the results after increasing the discount rate from $7 \%$ to $11 \%$. On average keeping the existing apple trees value is more than the value achieved by the uproot-plant or graft strategies. In this scenario, by assuming the same cost of $\$ 50$ for both apple types, on average, orchardists lose between $\$ 80.05$ and $\$ 157.50$ per hectare for Braeburn and 
TABLE 5.9: Volatility Change Sensitivity.

This tables presents the results of land use optimisation when volatility increases from $8 \%$ and $13 \%$ in Table 5.4 to $12 \%$ and $17 \%$ for Braeburn and Queen apple types respectively. I report key variables: land use for keeping the existing Braeburn and Queen apple trees (Tval), land use for the competing apple type by using the uproot-plant strategy (Uval), land use for the competing apple type by using the graft strategy (Gval), optimal value amongst all 3 options (optimal), and finally the percentage difference dollar value between the optimal land use and what orchardists choose (Diff). All values are scaled based on thousands dollar and hectares.

\begin{tabular}{lccccr}
\hline \multicolumn{1}{c}{ Variable } & N Obs & Mean & Std Deviation & Min & Max \\
\hline Keep the Existing Trees Value (Tval) & 5188 & 994.74 & 142.64 & 614.60 & 1329.44 \\
Uproot-Plant Value (Uval) & 5188 & 877.10 & 152.51 & 746.45 & 1163.07 \\
Grvaft Value (Gval) & 5188 & 935.67 & 198.98 & 299.90 & 1327.78 \\
Optimal & 5188 & 1019.29 & 145.31 & 746.45 & 1329.44 \\
(Optimal-Actual)/Actual & 5188 & 0.0027 & 0.06 & 0.00 & 0.28 \\
\hline
\end{tabular}

TABLE 5.10: Discount Rate Change Sensitivity.

This tables presents the results of land use optimisation when discount rate increases from $7 \%$ in Table 5.4 to 11\%. I report key variables: land use for keeping the existing Braeburn and Queen apple trees (Tval), land use for the competing apple type by using the uproot-plant strategy (Uval), land use for the competing apple type by using the graft strategy (Gval), optimal value amongst all 3 options (optimal), and finally the percentage difference dollar value between the optimal land use and what orchardists choose (Diff). All values are scaled based on thousands dollar and hectares.

\begin{tabular}{lccrrr}
\hline \multicolumn{1}{c}{ Variable } & N Obs & Mean & Std Deviation & Min & Max \\
\hline Keep the Existing Trees Value (Tval) & 5188 & 723.04 & 102.11 & 251.82 & 817.58 \\
Uproot-Plant Value (Uval) & 5188 & 537.71 & 46.25 & 480.11 & 605.09 \\
Grvaft Value (Gval) & 5188 & 625.55 & 110.20 & 93.18 & 736.04 \\
Optimal & 5188 & 735.10 & 77.33 & 480.11 & 817.58 \\
(Optimal-Actual)/Actual & 5188 & 0.0028 & 0.10 & 0.00 & 1.40 \\
\hline
\end{tabular}

between $\$ 105.03$ and \$307.01 per hectare for Queen over years 2006-16. 


\subsection{Conclusion}

In this chapter, I construct a real options switching problem by having two types of apple trees (Braeburn and Queen) with a finite life. My model optimises the land use policy with uncertainty and reversibility. I also model two competing switching strategies for orchardists to change their apple types. I implement my model for orchards in the Hawke's Bay region to examine if orchardists' behaviour follows the optimal land use policy that is proposed by my model. Orchardists have the option to keep the existing apple trees, or to switch to the competing apple type using the uprootplant or graft strategies. The former is a more expensive and slower process but results in a rejuvenated orchard whereas the latter is less expensive and faster but continues with the existing trees. My results suggest that orchardists employ the uproot-plant strategy when apple trees are no longer fully productive and are at the last stage of their life. In contrast, orchardists employ the graft strategy when orchards are young. In both strategies the cash flows begin to grow gradually until apple trees reach full productivity but the graft strategy has an earlier production than the uproot-plant strategy due to keeping the existing rootstock.

There are situations in my dataset where orchardists' actions do not match the optimal land use policy proposed by my real options model. According to the current model set up, on average an orchardist with a hectare land area loses $0.5 \%$ of the corresponding net revenue of land value that by assuming the same cost of $\$ 50$ for both apple types, varies from $\$ 220.93$ to $\$ 382.43$ for Braeburn and from $\$ 272.98$ to $\$ 693.77$ for Queen. Orchardists have a tendency towards keeping the existing trees and do not exercise their options to switch to the competing apple type either through the uproot-plant or the graft strategy. This hysteresis might govern their behaviour as long as there is not any force or a strong incentive to convince them to switch. Any financing or incentive from the government may lead orchardists to switch at the right time and decrease their inertia. My results 
from the conditional logit model show that orchardists are biased towards inertia. However, these results assume that orchardists do the right thing in the next period of time and theses costs would be small unless they are really in the money. The differences between the optimal land value based on the real options analysis and the actual value that orchardists achieve through their current policy are a proxy for how far they are from the policy advised by my model. However, the model results are based on some assumptions and changing the parameters will minimise the difference between optimal and actual. I test this assertion by changing the feeding parameters in sensitivity analysis section and find lower difference. This shows that orchardists might use different parameters than what I have used in my model. Also, a range of simplifications such as choosing only two apple types and ignoring the fact that orchardists have a portfolio of apple varieties might explain the hysteresis among orchardists that results in difference between theory and practice. Comparing small orchardists with large orchardists reveals the same attitude of being less willing to exercise switching options. 


\section{Bibliography}

Ahn, HeeJoon, Bae, KeeHong, and Chan, Kallok (2001). "Limit Orders, Depth, and Volatility: Evidence from the Stock Exchange of Hong Kong". In: The Journal of Finance 56.2, pp. 767-788.

Andersen, Torben and Bollerslev, Tim (1997). "Intraday periodicity and volatility persistence in financial markets". In: Journal of Empirical Finance 4.3, pp. 115-158.

- (1998). "Deutsche MarkDollar Volatility: Intraday Activity Patterns, Macroeconomic Announcements, and Longer Run Dependencies". In: The Journal of Finance 53.1, pp. 219-265.

Andersen, Torben, Bollerslev, Tim, Diebold, Francis, and Vega, Clara (2003). "Micro Effects of Macro Announcements: Real-Time Price Discovery in Foreign Exchange". In: American Economic Review 93.1, pp. 38-62.

- (2007). "Real-time price discovery in global stock, bond and foreign exchange markets". In: Journal of International Economics 73.2, pp. 251277.

Anderson, Kim and Brorsen, Wade (2005). "Marketing Performance of Oklahoma Farmers". In: American Journal of Agricultural Economics 87.5, pp. $1265-1270$.

ANZ Agri Focus (2015). New Zealand Economics, pp. 1-41.

Balduzzi, Pierluigi, Elton, Edwin, and Green, Tony (2001). "Economic News and Bond Prices: Evidence from the US. Treasury Market". In: The Journal of Financial and Quantitative Analysis 36.4, pp. 523-543. 
Bauwens, Luc, Ben-Omrane, Walid, and Giot, Pierre (2005). “News announcements, market activity and volatility in the euro/dollar foreign exchange market". In: Journal of International Money and Finance 24.7, pp. 1108-1125.

Ben-Omrane, Walid and Hafner, Christian (2015). "Macroeconomic News Surprises and Volatility Spillover in the Foreign Exchange Markets". In: Empirical Economics 48.2, pp. 577-607.

Bensoussan, Alain, Hoe, SingRu, Yan, ZhongFeng, and Yin, George (2017). "Real Options with Competition and Regime Switching". In: Mathematical Finance 27.1, pp. 224-250.

Bollen, Nicolas (1999). "Real Options and Product Life Cycles". In: Management Science 45.5, pp. 621-769.

Boudt, Kris, Neely, Christopher, Secru, Piet, and Wauters, Marjan (2017). "The response of multinationals foreign exchange rate". In: Working Paper.

Brekke, Kjell and Oksendal, Bernt (1988). "Anat R. Admati and Paul Pfleiderer". In: The Review of Financial Studies 1.1, pp. 3-40.

Brennan, Michael and Schwartz, Eduardo (1985). "Evaluating Natural Resource Investments". In: The Journal of Business 58, pp. 135-157.

Brounen, Dirk, Jong, Abe de, and Koedijk, Kees (2004). “Corporate Finance in Europe: Confronting Theory with Practice". In: Financial Management 33.4, pp. 71-101.

Brunzell, Tor, Liljeblom, Eva, and Vaihekoski, Mika (2013). “Accounting and Finance". In: Financial Management 53.1, pp. 85-110.

Bulan, Laarni, Mayer, Christopher, and Somerville, Tsuriel (2009). "Irreversible investment, real options, and competition: Evidence from real estate development". In: Journal of Urban Economics 65, pp. 237-251.

Carr, Peter (2009). "The Valuation of Sequential Exchange Opportunities". In: The Journal of Finance 43.5, pp. 1235-1256.

Cetinkaya, Elcin and Thiele, Aurlie (2014). "Real Options: A Survey ". In: Lehigh University. 
Chordia, Tarun, Roll, Richard, and Subrahmanyam, Avanidhar (2002). "Order imbalance, liquidity, and market returns". In: Journal of Financial Economics 65.1, pp. 111-130.

Clare, Andrew and Courtenay, Roger (2001). "Assessing the impact of macroeconomic news announcements on securities prices under different monetary policy regimes". In: Bank of England working papers.

Clewlow, Les and Strickland, Chris (1998). Implementing Derivatives Model. West Sussex PO19 IUD, England: John Wiley and Sons Ltd.

Cunha-e-sa, Maria and Franco, Sofia (2016). "The Effects of Development Constraints on Forest Management at the Urban-Forest Interface". In: American Journal of Agricultural Economics 99.3, pp. 614-636.

Cyr, Don, Hanagriffb, Roger, and Kwongc, Lester (2010). “What is Making Investment in the Texas Vineyard Industry Tick? A Real Options Analysis of Entry and Exit". In: Journal of Wine Economics 5.2, pp. 236255.

Davis, Graham and Cairns, Robert (2017). “The Odd Notion of "Reversible Investment"'. In: Journal of Banking and Finance 815, pp. 172-180.

Deaton, Angus and Laroque, Guy (1992). "On the Behaviour of Commodity Prices". In: The Review of Economic Studies 59.1, pp. 1-23.

Dixit, Avinash (1992). "Investment Hysteresis". In: Journal of Economic Perspectives 6, pp. 107-132.

Douglas, Jim and Rachford, Henry (1955). “On the Numerical Solution of Heat Conduction Problems in Two and Three Space Variables". In: The Transactions of the American Mathematical Society 82.2, pp. 421-489.

Ederington, Louis and Lee, Jae Ha (1995). "The Short-Run Dynamics of the Price Adjustment to New Information". In: The Journal of Financial and Quantitative Analysis 30.1, pp. 1161-1191.

Evans, Martin and Lyons, Richard (2002). “Order Flow and Exchange Rate Dynamics". In: Journal of Political Economy 110.1, pp. 170-180.

- (2005). "Do Currency Markets Absorb News Quickly". In: Journal of International Money and Finance 24, pp. 197-217. 
Evans, Martin and Lyons, Richard (2008). "How is macro news transmitted to exchange rates?" In: Journal of Financial Economics 88.1, pp. 2650.

Ewald, Christian-Oliver, Ouyang, Ruolan, and Siu, Tak-Kuen (2016). “On the Market Consistent Valuation of Fish Farms: Using the Real Option Approach and Salmon Futures". In: American Journal of Agricultural Economics 99.1, pp. 207-224.

Fackler, Paul and Livingston, Michael (2002). “Optimal Storage by Crop Producers". In: American Journal of Agricultural Economics 84.3, pp. 645659.

Fleming, Michael and Remolona, Eli (1999). "Price Formation and Liquidity in the US. Treasury Market: The Response to Public Information". In: The Journal of Finance 54.5, pp. 1901-1915.

Forsberg, Lars and Ghysels, Eric (2007). "Why Do Absolute Returns Predict Volatility So Well?" In: Journal of Financial Econometrics 5.1, pp. 3167.

Foucault, Thierry, Hombert, Johan, and Rosu, Ioanid (2016). "News trading and speed". In: The Journal of Finance 71.1, pp. 335-382.

Foucault, Thierry, Moinas, Sophie, and Theissen, Erik (2007). “Does Anonymity Matter in Electronic Limit Order Markets?" In: The Review of Financial Studies 20.5, pp. 1707-1747.

Frijns, Bart and Tourani-Rad, Alireza (2016). "The long-run performance of the New Zealand stock markets: 1899-2013". In: Pacific Accounting Review 28.1, pp. 59-70.

Gomes, Petro and Peraita, Elena Vozmediano (2016). “The Effects of Announcements of Leading and Sentiments Indicators on Euro Area Financial Markets". In: Working Paper.

Gosnell, Thomas, Keown, Arthur, and Pinkerton, John (1996). "The intraday speed of stock price adjustment to major dividend changes: Bidask bounce and order flow imbalances". In: Journal of Banking and Finance 20.2, pp. 247-266. 
Granger, Clive and Sin, ChorYiu (2000). "Modelling the absolute returns of different stock indices: exploring the forecastability of an alternative measure of risk". In: Journal of Forecasting 19.4, pp. 277-298.

Guthrie, Graeme (2007). “Missed Opportunity: Optimal Investment Timing When Information is Costly". In: Journal of Financial And Quantitative Analysis 42.2, pp. 467-488.

Hagedorn, Lewis, Irwin, Scott, Good, Darrel, and Colino, Evelyn (2005). "Does the Performance of Illinois Corn and Soybean Farmers Lag the Market?" In: American Journal of Agricultural Economics 87.5, pp. 12711279.

Hansen, Peter and Lunde, Asger (2006). "Realized Variance and Market Microstructure Noise". In: Journal of Business and Economic Statistics 24.2, pp. 127-161.

Hasbrouck, Joel (1993). "Assessing the Quality of a Security Market: A New Approach to Transaction-Cost Measurement". In: The Review of Financial Studeis 6.1, pp. 191-212.

Hauer, Grant, Luckert, Marty, Yemshanov, Denys, and Unterschultz, Jim (2016). “A Spatial Real Options Approach for Modeling Land Use Change: Assessing the Potential for Poplar Energy Plantations in Alberta". In: Canadian Journal of Agricultural Economics 65, pp. 271-292.

Hoffmann, Peter (2014). "A Dynamic Limit Order Market with Fast and Slow Traders". In: Journal of Financial Economics 113.1, pp. 156-169.

Horticulture, New Zealand Horticulture (2017). Fresh Facts.

Hossiso, Kassu Wamisho and Ripplinger, David (2017). “The Value of Switching Production Options in a Flexible Biorefinery". In: Agricultural and Resource Economics Review 46.1, pp. 146-173.

Hyde, Jeffrey, Stokes, Jeffrey, and Engel, Phoebe (2003). “Optimal Investment in an Automatic Milking System: an Application of Real Options". In: Journal of Agricultural Finance Review 63, pp. 75-92.

Ihli, Hanna, Maart-Noelck, Syster, and Musshoff, Oliver (2013). "Does timing matter? A real options experiment to farmers' investment and dis- 
investment behaviour". In: The Australian Journal of Agriculture and Resource Economics 58, pp. 430-452.

James, Laura, Swinton, Scott, and Thelen, Kurt (2003). "Profitability Analysis of Cellulosic Energy Crops Compared with Corn". In: Agronomy journal 102.2, pp. 675-687.

Jiang, George, Lo, Ingrid, and Valente, Giorgio (2014). "High-Frequency trading around macroeconomic news announcements: evidence from the US. treasury market". In: Working Paper 56.

Jones, Charles, Kaul, Gautam, and Lipson, Marc (1994). “Transactions, Volume, and Volatility". In: Review of Financial Studies 7.4, pp. 156-169.

Kennedy, John (1986). Dynamic programming : Applications to Agriculture and Natural Resources. London ; New York: Elsevier Applied Science Publishers.

Kim, Hyun Seok and Brorsen, Wade (2012). "Can Real Option Values Explain Apparent Storage at a Loss". In: Journal of Applied Economics 44.16, pp. 2081-2090.

Kim, Ha, Sooji, and Hyoungkwan (2017). “Using Real Options for Urban Infrastructure Adaptation under Climate Change". In: Journal of Cleaner Production 143, pp. 40-50.

King, Michael, Osler, Carol, and Rime, Dagfinn (2013). “The Market Microstructure Approach to Foreign Exchange: Looking Back and Looking Forward". In: Journal of International Money and Finance 38, pp. 95119.

Kliber, Agata and Bedowska-Sojka, Barbara (2010). "Realized Volatility Versus GARCH and Stochastic Volatility Models. The Evidence from the WIG20 index and the EUR/PLN Foreign Exchange Market". In: Przegld statystyczny/Statistical Review 4, pp. 105-127.

Kozlova, Mariia (2017). "Real Option Valuation in Renewable Energy Literature: Research Focus, Trends and Design". In: Renewable and Sustainable Energy Reviews 80, pp. 180-196. 
Kyle, Albert (1985). "Continuous auctions and insider trading". In: Econometrica 53.6, pp. 1315-1335.

Lambrecht, Bart (2017). "Real Options in Finance". In: Journal of Banking and Finance 81, pp. 166-171.

Livingston, Michael, Roberts, Michael, and Zhang, Yue (2014). “Optimal Sequential Planting of Corn and Soybeans under Price Uncertainty". In: American Journal of Agricultural Economics 97.3, pp. 855-878.

Lo, Ingrid and Sapp, Stephen (2008). "The Submission of Limit Orders or Market Orders: The Role of Timing and Information in the Reuters D2000-2 System". In: Journal of International Money and Finance 27.7, pp. 1056-1073.

- (2010). "Order aggressiveness and quantity: How are they determined in a limit order market?" In: Journal of International Financial Markets, Institutions and Money 20.3, pp. 213-237.

- (2011). "Belief dispersion and order submission strategies in the foreign exchange market". In: Working Paper Bank of Canada 8.

Lovcha, Yuliya and Perez-Laborda, Alejandro (2013). "Is exchange rate Customer order flow relationship linear? Evidence from the Hungarian FX market". In: Journal of International Money and Finance 35, pp. 20-35.

Majd, Saman and Pindyck, Robert (1987). "Time to build, option value, and investment decisions". In: Journal of Financial Economics 18, pp. 727.

McDonald, Robert and Siegel, Daniel (1985a). "Investment and the Valuation of Firms When There Is an Option to Shut Down". In: International Economic Review 101.2, pp. 331-349.

- (1985b). "The Value of Waiting to Invest". In: Quarterly Journal of Economics 101, pp. 707-727.

Milne, Alistair and Whalley, Elizabeth (2000). "Time to build, option value and investment decisions". In: Journal of Financial Economics 56.2, pp. 325332.

MPI, Ministry for Primary Industry (2017). Pipfruit Monitoring Programme. 
Myers, Stewart (1977). “Determinants of corporate borrowing”. In: Journal of Financial Economics 5.2, pp. 147-175.

Neely, Christopher and Dey, Rubun (2010). "A survey of announcement effects on foreign exchange returns". In: Federal Reserve Bank of St. Louis 92.5, pp. 417-464.

Newey, Whitney and West, Kenneth (1987). "A Simple, Positive Semi-definite, Heteroskedasticity and Autocorrelation Consistent Covariance Matrix". In: Econometrica 55.3, pp. 703-08.

Novy-Marx, Robert (2007). “An Equilibrium Model of Investment Under Uncertainty". In: The Review of Financial Studies 20.5, pp. 1461-1502.

Odeninga, Martin, Muhoff, Oliver, and Balmannb, Alfons (2005). "Investment Decisions in Hog Finishing: An Application of the Real Options Approach". In: Agricultural Economics 32, pp. 47-60.

Pasquariello, Paolo and Vega, Clara (2007). "Informed and Strategic Order Flow in the Bond Markets". In: Review of Financial Studies 20.6, pp. 1975-2019.

Peaceman, Donald and Rachford, Henry (1955). “The Numerical Solution of Parabolic and Elliptic Differential Equations". In: Journal of the Society for Industrial and Applied Mathematics 3.1, pp. 28-41.

Pederson, Glenn and Zou, Tianyu (2008). “Using real options to evaluate ethanol plant expansion decisions". In: Agricultural Finance Review 69.1, pp. 23-35.

Purvis, Amy, Boggess, William, Moss, Charles, and Holt, John (1995). “Technology adoption decisions under irreversibility and uncertainty: an ex ante approach". In: American Journal of Agricultural Economics 77, pp. 541-51.

Rebiasz, Bogdan, Gawel, Bartlomiej, and Skalna, Iwona (2017). “Valuing Managerial Flexibility : an Application of Real-Option Theory to Steel Industry Investments". In: Operations Research and Decisions 27.2, pp. 91111. 
Rime, Dagfinn, Sarno, Lucio, and Sojli, Elvira (2010). "Exchange rate forecasting, order flow and macroeconomic information". In: Journal of International Economics 80.1, pp. 72-88.

Sager, Michael and Taylor, Mark (2011). "Under the microscope: the structure of the foreign exchange market". In: International Journal of Finance and Economics 11.1, pp. 81-95.

Savolainen, Jyrki (2016). "Real Options in Metal Mining Project Valuation: Review of Literature". In: Resources Policy 50, pp. 49-65.

Schmit, Todd, Luo, Jianchuan, and Conrad, Jon (2011). "Estimating the influence of US ethanol policy on plant investment decisions: A real options analysis with two stochastic variables". In: Energy Economics 33.6, pp. 1194-1205.

Schmit, Todd, Luo, Jianchuan, and Tauer, Loren (2009). "Ethanol Plant Investment using Net Present Value and Real Options Analyses". In: Biomass Bioenergy 33, pp. 1442-1451.

Seyoum-Tegegn, Emayenesh and Chan, Chris (2013). "What Is Making Vineyard Investment in Northwest Victoria Australia, Slow to Adjust?" In: Journal of Wine Economics 8.1, pp. 3-102.

Shah, Payal and Ando, Amy (2016). "Permanent and Temporary Policy Incentives for Conservation under Stochastic Returns from Competing Land Use". In: American Journal of Agricultural Economics 4, pp. 10741094.

Sina, Matias and Guzman, Juan (2019). "Real Option Valuation of Open Pit Mines with Two Processing Methods". In: Journal of Commodity Markets 13, pp. 30-39.

Slade, Margaret (2001). "Valuing Managerial Flexibility: An Application of Real-Option Theory to Mining Investments". In: Journal of Environmental Economics and Management 41.2, pp. 193-233.

Song, Feng, Zhao, Jinhua, and Swinton, Scott (2011). "Switching to Perennial Energy Crops Under Uncertainty and Costly Reversibility". In: American Journal of Agricultural Economics 93.3, pp. 768-783. 
Tang, Bao, Zhou, Hui, Chen, Hao, Wang, Kai, and Cao, Hong (2017). “Investment Opportunity in China's Overseas Oil Project: An Empirical Analysis Based on Real Option Approach". In: Energy Policy 105, pp. 1726.

Tauer, Loren (2006). “When to Get In and Out of Dairy Farming: A Real Option Analysis". In: Agricultural and Resource Economics Review 35.2, pp. 339-347.

Trigeorgis, Lenos and Tsekrekos, Andrianos (2018). "Real Options in Operations Research: A Review". In: European Journal of Operational Research 270, pp. 1-24.

Tubetov, Dulat, Musshoff, Oliver, and Kellner, Ulla (2012). “Investments in Kazakhstani Dairy Farming: A Comparison of Classical Investment Theory and the Real Options Approach". In: Quarterly Journal of International Agriculture 51.3, pp. 257-284.

Tyner, Wallace (2008). "The US Ethanol and Biofuels Boom: Its Origins, Current Status, and Future Prospects". In: BioScience 58.7, pp. 646-653.

Wang, Xiping and Du, Lei (2016). "Study on Carbon Capture and Storage (CCS) Investment Decision-making Based on Real Options for China's Coal-fired Power Plants". In: Annals of Economics and Finance 112, pp. 41234131.

Williams, Jeffery and Wright, Brian (1991). Storage and Commodity Markets. Cambridge University Press.

Winter-Nelson, Alex and Amegbeto, Koffi (1998). “Option values to conservation and agricultural price policy: application to terrace construction in Kenya". In: American Journal of Agricultural Economics 80, pp. 40918.

Zhang, Mingming, Zhou, Peng, and Zhou, Degun (2016). “A Real Options Model for Renewable Energy Investment with Application to Solar Photovoltaic Power Generation in China". In: Energy Economics 59, pp. 213-226. 